\title{
Synthesis and Characterisation of Photocatalyst Silver/Silver Halide Nanocomposites
}

By

Eldon Warwick Tate

\author{
A thesis \\ submitted to the Victoria University of Wellington \\ in partial fulfilment of the requirements for the degree of \\ Master of Science in Chemistry
}

Victoria University of Wellington 


\section{Abstract}

The photochemical activity of silver halides forms the basis of photography and latent image formation. More recently it has been used to create hybrid silver/silver halide nanoparticles. These are formed through partial reduction of $\mathrm{Ag}^{+}$to $\mathrm{Ag}^{0}$ by a photochemical selfsensitisation when irradiated with light. This gives the silver/silver halide nanoparticles interesting photocatalytic properties. As such, these silver/silver halide nanoparticles have seen to be part of group of photocatalysts known as plasmonic photocatalysts. Where, the photocatalytic mechanism is enhanced by the surface plasmon resonance of noble metal nanodomains on the surface of the silver halide nanoparticle.

The silver/silver halide nanoparticles of $\mathrm{Cl}^{-}, \mathrm{Br}^{-}$and $\mathrm{I}^{-}$were synthesised and characterised. Silver/silver halide nanoparticles were then incorporated into porous support materials creating silver/silver halide nanocomposite materials. This was through a straight forward aqueous synthesis method, where silver halide nanoparticles precipitated from solution, and nanoparticle size, shape and stabilisation was controlled by the porous support material. Silver/silver halide nanocomposite samples using $\mathrm{Cl}^{-}, \mathrm{Br}^{-}$and $\mathrm{I}^{-}$were synthesised using wool fibres, kraft paper fibres and nanostructured calcium silicate as supports.

UV/Vis and XRD showed $\mathrm{Ag}^{0}$ nanodomains were formed during the self-sensitisation process. SEM showed the morphology of the nanocomposites and that the nanoparticles were distributed within the nanocomposite matrix, not deposited on the surface. Preliminary photocatalytic activity of $\mathrm{Ag} / \mathrm{AgCl}$ nanoparticles and nanocomposites was evaluated through the degradation of methylene blue when irradiated with light. All samples showed increased photocatalytic activity with the $\mathrm{Ag} / \mathrm{AgCl}$ nanoparticles. 


\section{Acknowledgements}

Firstly, I would like to thank my supervisor Prof. Jim Johnston who has been an inspiration throughout this entire research programme. The direction and assistance has been great. I look forward to working with you again in the future. The research group have been a fantastic group of people to work with. Thank you very much to Kerstin and Matthew for dealing with my questions and problems. The rest of you, I have had fantastic conversations with you all and thank you for the support. Maria and Thomas you can be bad influences, but at the same time amazing help.

Thank you to the rest of SCPS also. I would like to mention David Flynn, his SEM and TEM expertise was invaluable. Alec La Grow for his help with electron diffraction and some of the more difficult TEM work. Apologies to anyone I have missed, rest assured that I am very thankful. I would like to thank my family and friends, Mum and Dad for emergency funds and a lot of help and motivation, and Hannah for proof reading a big bunch of science that meant nothing to you.

Finally I would like to thank Prof. Jim Johnston again, for the financial support for this project and the Curtis-Gordon Research Scholarship in Chemistry, this has been greatly appreciated. 


\section{Contents}

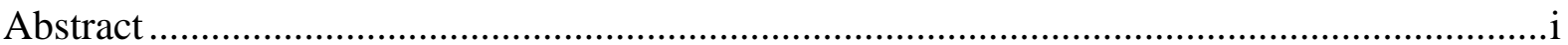

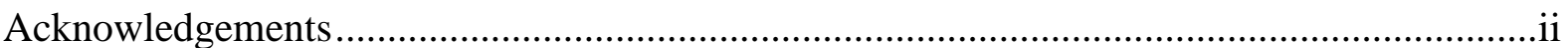

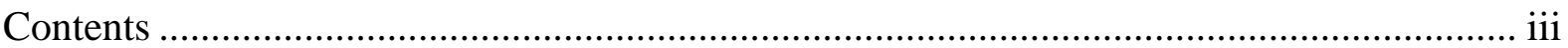

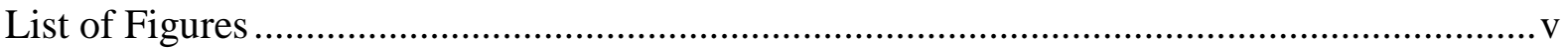

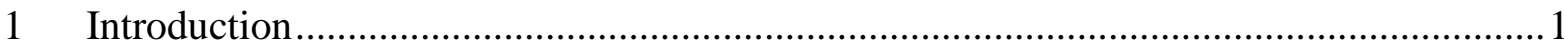

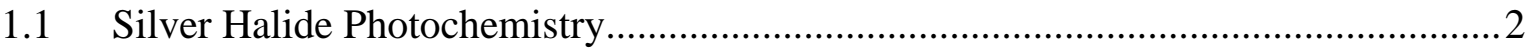

1.2 Silver Halide Nanoparticle Synthesis ................................................................

1.3 Metal Nanoparticles and Surface Plasmon Resonance ..........................................5

$1.4 \quad$ Silver Nanoparticle Surface Plasmon Resonance ...............................................

1.5 Silver/Silver Halide Plasmonic Photocatalysts ...................................................... 10

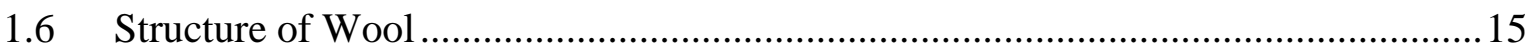

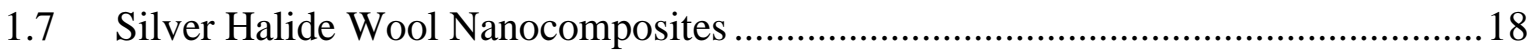

$1.8 \quad$ Bleached and Unbleached Kraft Paper Fibres....................................................... 20

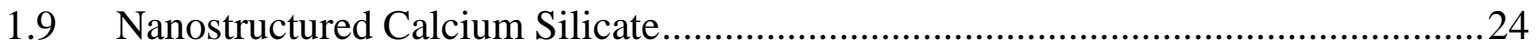

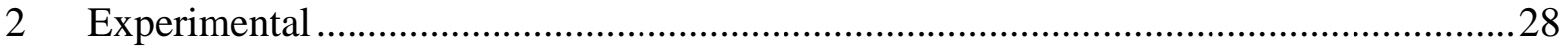

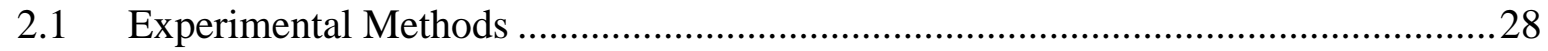

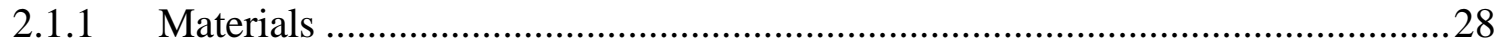

2.1.2 Preparation of silver/silver halide colloids .................................................28

2.1.3 Preparation of silver/silver halide wool nanocomposites ................................29

2.1.4 Preparation of silver/silver halide kraft fibre nanocomposites ......................... 30

2.1.5 Synthesis of silver/silver halide nanostructured calcium silicate nanocomposites

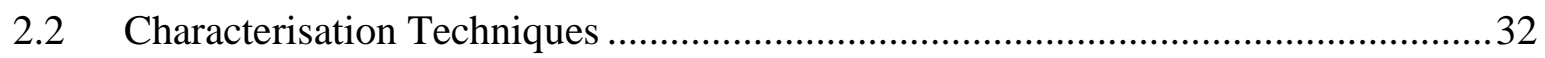

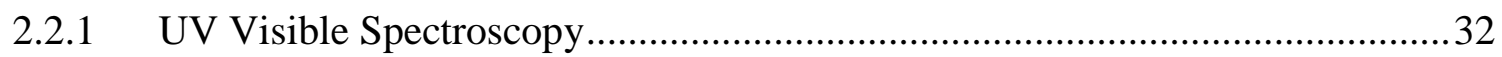

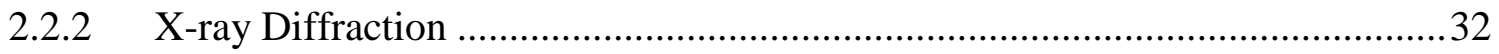




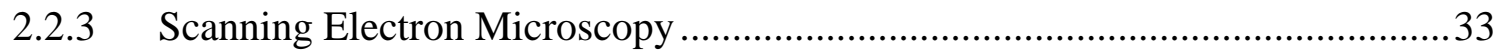

2.2.4 Energy Dispersive X-ray Spectroscopy ….......................................................... 33

2.2.5 Transmission Electron Microscopy …............................................................ 33

2.2.6 Determination of Photocatalyic Activity ......................................................... 34

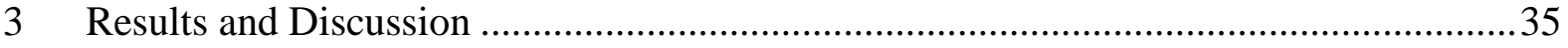

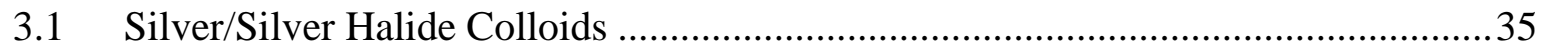

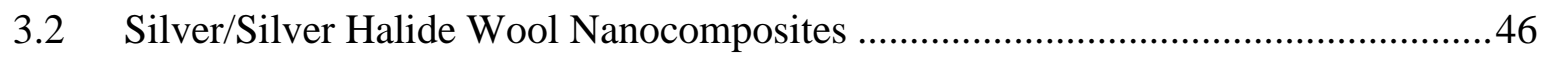

3.3 Silver/Silver Halide Kraft Fibre Nanocomposites ................................................59

3.4 Silver/Silver Halide Nanostructured Calcium Silicate Nanocomposites ...................72

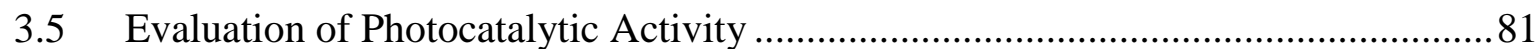

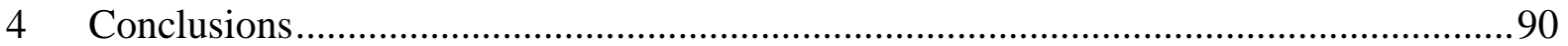

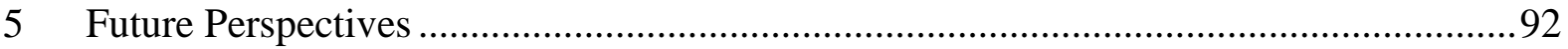

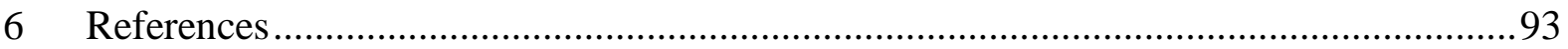

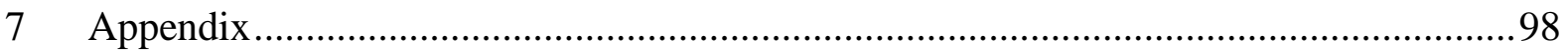




\section{List of Figures}

Figure 1 Lycurgus cup in reflected (a) and transmitted (b) light........................................5

Figure 2 Plasmon oscillation in metal nanospheres. .........................................................6

Figure 3 Interaction of the electromagnetic field of incoming light with a) spherical

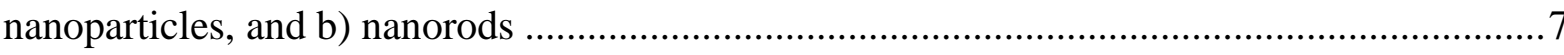

Figure 4 Absorption profile of $\mathrm{Ag}$ nanorods with increasing aspect ratios ............................. 8

Figure 5 Size and shape of silver nanoparticles correlated with absorbed wavelength of light

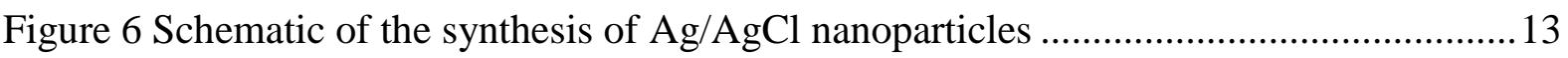

Figure 7 Scheme showing the structure of $\mathrm{Ag} / \mathrm{AgCl}$ nanoparticle and radical formation....... 14

Figure 8 Bonds liking adjacent polypeptide chains in wool $\alpha$-keratin fibres. ........................ 16

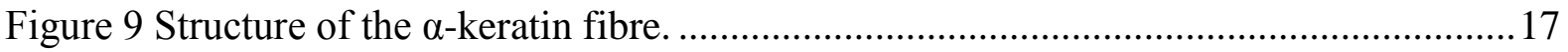

Figure 10 Types of medulla that can occur in wool fibres. a) Fragmental, b) interrupted and c)

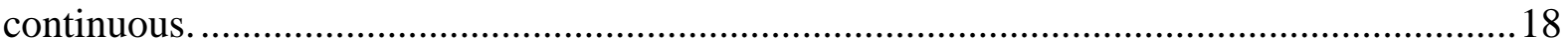

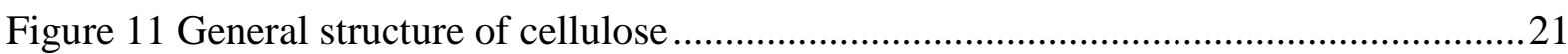

Figure 12 Example of a softwood lignin fragment ...........................................................23

Figure 13 SEM image of the gypsum desert rose structure of NCS ....................................25

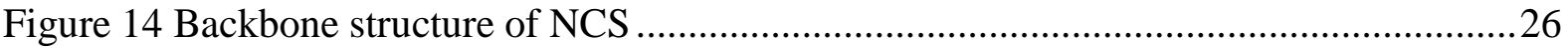

Figure $15 \mathrm{UV} / \mathrm{Vis}$ spectra of $\mathrm{AgCl}$ colloid before and after light exposure ............................38

Figure $16 \mathrm{UV} / \mathrm{Vis}$ spectra of $\mathrm{AgBr}$ colloid before and after light exposure ............................38

Figure 17 UV/Vis spectra of AgI colloid before and after light exposure.............................39

Figure $18 \mathrm{XRD}$ pattern of $\mathrm{AgCl}$ colloid before light exposure ............................................ 40

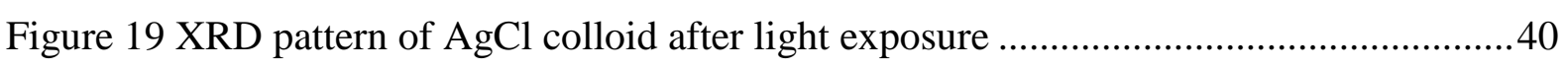

Figure 20 XRD pattern of $\mathrm{AgBr}$ colloid after light exposure ............................................. 41

Figure 21 XRD pattern of AgI colloid after light exposure ............................................... 41

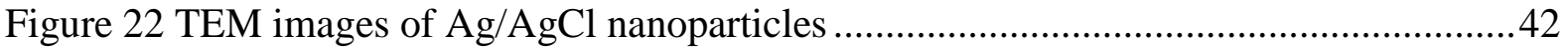

Figure $23 \mathrm{TEM}$ of an $\mathrm{Ag} / \mathrm{AgCl}$ nanoparticle over time A) $0 \mathrm{~s} \mathrm{~B}) 30 \mathrm{~s} \mathrm{C}) 60 \mathrm{~s} \mathrm{D)} 120 \mathrm{~s} . . . . . .43$

Figure $24 \mathrm{AgCl}$ exposed to light nanoparticle A) TEM image of diffraction sample B)

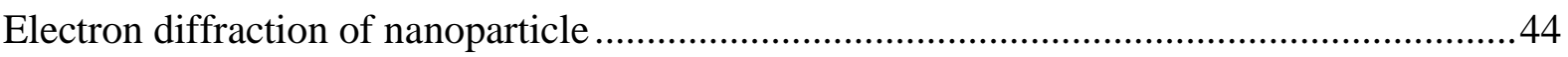

Figure 25 STEM EDS of TEM AgCl nanoparticle sample ................................................ 45

Figure $26 \mathrm{UV} / \mathrm{Vis}$ Spectra of $\mathrm{AgCl}$ wool nanocomposite before and after light exposure ..... 47

Figure $27 \mathrm{UV} /$ Vis Spectra of AgBr wool nanocomposite before and after light exposure .....47 
Figure $28 \mathrm{UV} / \mathrm{Vis}$ Spectra of AgI wool nanocomposite before and after light exposure .48

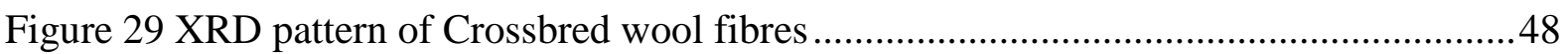

Figure $30 \mathrm{XRD}$ pattern of $\mathrm{AgCl}$ Wool Nanocomposite without light exposure......................49

Figure $31 \mathrm{XRD}$ pattern of $\mathrm{AgCl}$ Wool Nanocomposite after light exposure ..........................50

Figure 32 XRD pattern of AgBr Wool Nanocomposite without light exposure .....................50

Figure 33 XRD pattern of AgBr Wool Nanocomposite after light exposure .........................51

Figure 34 XRD pattern of AgI wool nanocomposite without light exposure ..........................51

Figure 35 XRD pattern of AgI wool nanocomposite after light exposure ..............................51

Figure $36 \mathrm{XRD}$ pattern of $\mathrm{AgCl}$ wool nanocomposite after light exposure ...........................52

Figure $37 \mathrm{XRD}$ pattern of $\mathrm{AgCl}$ wool nanocomposite after 5 days light exposure in $\mathrm{Xe}$ Arc lamp.

Figure $38 \mathrm{SEM}$ images of $\mathrm{AgCl}$ wool nanocomposite A) SEI image B) Backscatter image ..53 Figure 39 SEM images of $\mathrm{AgBr}$ wool nanocomposite A) SEI image B) Backscatter image..54 Figure 40 SEM Images of AgI wool nanocomposite A) SEI image B) Backscatter image ....54 Figure $41 \mathrm{AgCl}$ wool nanocomposite A) Backscatter SEM image B) EDS map of Cl C) EDS map of Ag D) EDS overlay.....

Figure $42 \mathrm{AgBr}$ wool nanocomposite A) SEM backscatter image B) EDS map of Br C) EDS map of Ag D) EDS overlay..... .55

Figure 43 AgI wool nanocomposite A) SEM backscatter image B) EDS map of I C) EDS map of Ag D) EDS overlay..... . .56

Figure $44 \mathrm{AgCl}$ wool cross section A) SEM backscatter image B) EDS map of Cl C) EDS map of Ag D) EDS overlay.....

Figure $45 \mathrm{AgBr}$ wool cross section A) SEM backscatter image B) EDS map of Br C) EDS map of Ag D) EDS overlay.....

Figure 46 AgI wool nanocomposite cross section A) SEI image B) Backscatter image.........58

Figure 47 Representation of the formation mechanism of the $\mathrm{Ag} / \mathrm{AgCl}$ wool nanocomposite

Figure $48 \mathrm{UV} / \mathrm{Vis}$ of $\mathrm{AgCl}$ unbleached kraft fibres after light exposure..... 60

Figure $49 \mathrm{UV} / \mathrm{Vis}$ of $\mathrm{AgBr}$ unbleached kraft fibres with light exposure. 61

Figure $50 \mathrm{UV} / \mathrm{Vis}$ of AgI unbleached kraft fibres with light exposure ................................. 61

Figure $51 \mathrm{UV} / \mathrm{Vis}$ of $\mathrm{AgCl}$ Bleached kraft Fibres after light exposure .................................62

Figure $52 \mathrm{UV} / \mathrm{Vis}$ of $\mathrm{AgBr}$ Bleached kraft Fibres after light exposure .................................63

Figure $53 \mathrm{UV/Vis}$ of AgI Bleached kraft Fibres after light exposure ....................................63

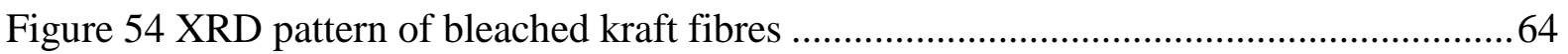


Figure $55 \mathrm{XRD}$ pattern of $\mathrm{AgCl}$ bleached kraft nanocomposite. .65

Figure 56 XRD pattern of $\mathrm{AgBr}$ bleached kraft nanocomposite 65

Figure 57 XRD pattern of AgI bleached kraft nanocomposite

Figure 58 SEM images of $\mathrm{AgCl}$ bleached kraft fibre nanocomposite A) SEI image B)

Backscatter image ..... .66

Figure 59 SEM images of AgBr bleached kraft fibre nanocomposite A) SEI image B)

Backscatter image

Figure 60 SEM images of AgI bleached kraft nanocomposite A) SEI image B) Backscatter image.

Figure $61 \mathrm{AgCl}$ kraft nanocomposite A) SEM backscatter image B) EDS map of Cl C) EDS map of Ag D) EDS overlay..... .68

Figure $62 \mathrm{AgBr}$ kraft nanocomposite A) SEM backscatter image B) EDS map of Br C) EDS map of Ag D) EDS overlay. .68

Figure 63 AgI kraft nanocomposite A) SEM backscatter image B) EDS map of I C) EDS map of $\mathrm{Ag} \mathrm{D})$ EDS overlay.

Figure $64 \mathrm{AgCl}$ kraft cross section A) SEM backscatter image B) EDS map of Cl C) EDS map of Ag D) EDS overlay..... .70

Figure $65 \mathrm{AgBr}$ kraft cross section A) SEM backscatter image B) EDS map of Br C) EDS map of Ag D) EDS overlay.....

Figure 66 AgI kraft cross section A) SEM backscatter image B) EDS map of I C) EDS map of $\mathrm{Ag} \mathrm{D})$ EDS overlay. .71

Figure $67 \mathrm{UV} / \mathrm{Vis}$ spectra of $\mathrm{AgCl}$ NCS nanocomposite after light exposure .......................74

Figure $68 \mathrm{UV} / \mathrm{Vis}$ spectra of AgBr NCS nanocomposite after light exposure ....................... 74

Figure $69 \mathrm{UV} / \mathrm{Vis}$ spectra of AgI NCS nanocomposite after light exposure...........................75

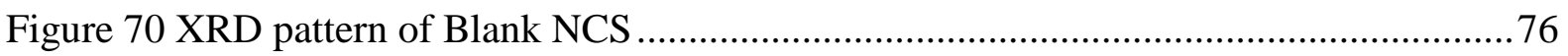

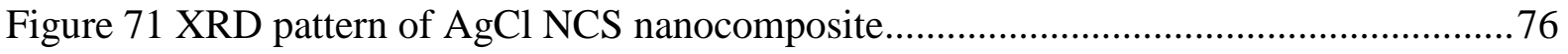

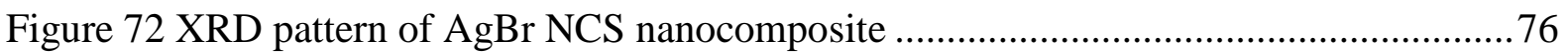

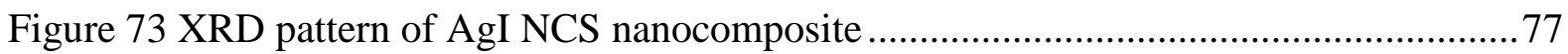

Figure 74 SEM images of AgCl NCS nanocomposite A) SEI image B) Backscatter image .. 78 Figure 75 SEM images of AgBr NCS nanocomposite A) SEI image B) Backscatter image .. 78 Figure 76 SEM images of AgI NCS nanocomposite A) SEI image B) Backscatter image.....78 Figure $77 \mathrm{AgCl}$ NCS nanocomposite A) SEM backscatter image B) EDS map of Cl C) EDS map of Ag D) EDS overlay..... 
Figure $78 \mathrm{AgBr}$ NCS nanocomposite A) SEM backscatter image B) EDS map of Br C) EDS map of Ag D) EDS overlay

Figure 79 AgI NCS nanocomposite A) SEM backscatter image B) EDS map of I C) EDS map

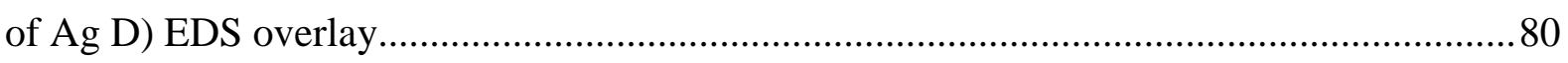

Figure $80 \mathrm{UV} / \mathrm{Vis}$ spectra of $\mathrm{MB}$ with bulk $\mathrm{AgCl}$ in $75 \mathrm{Xe}$ arc lamp overtime (min) ............83 Figure $81 \mathrm{UV} / \mathrm{Vis}$ spectra of $\mathrm{MB}$ with $\mathrm{Ag} / \mathrm{AgCl}$ nanoparticles in $75 \mathrm{~W} \mathrm{Xe}$ arc lamp overtime (min) .83

Figure 82 Decrease in MB over time .84

Figure $83 \mathrm{UV} / \mathrm{Vis}$ spectra of $\mathrm{MB}$ with $\mathrm{Ag} / \mathrm{AgCl}$ wool nanocomposite in $75 \mathrm{~W} \mathrm{Xe}$ arc lamp overtime ( $\mathrm{min})$ .85

Figure 84 Decrease in MB over time for wool samples .86 Figure $85 \mathrm{UV} / \mathrm{Vis}$ spectra of $\mathrm{MB}$ with $\mathrm{Ag} / \mathrm{AgCl} \mathrm{kraft}$ nanocomposite in $75 \mathrm{~W} \mathrm{Xe}$ arc lamp overtime (min) .87

Figure 86 Decrease in MB over time for kraft samples .87

Figure $87 \mathrm{UV} / \mathrm{Vis}$ spectra of $\mathrm{MB}$ with $\mathrm{Ag} / \mathrm{AgCl} \mathrm{NCS}$ nanocomposite in $75 \mathrm{~W} \mathrm{Xe}$ arc lamp overtime ( $\min )$ .88

Figure 88 Decrease in MB over time for NCS samples. .89 


\section{Introduction}

This research programme is focused on the synthesis and characterisation of silver/silver halide nanocomposites. Silver/silver halide nanoparticles are formed from the partial conversion from the $\mathrm{Ag}^{+}$of silver halide nanoparticles to $\mathrm{Ag}^{0}$. The photochemistry of silver halides plays an important role in the synthesis of these unique composite nanoparticles. Hence an understanding of the photographic process and silver halide photochemistry is vital. This is introduced here before exploring silver halide nanoparticles and their synthesis as they form the basis for the silver/silver halide nanoparticles.

The partial conversion of $\mathrm{Ag}^{+}$to $\mathrm{Ag}^{0}$ to form silver/silver halide nanoparticles gives rise to nanodomains of silver, these have interesting optical properties. An introduction to surface plasmon resonance is required to explain these optical properties, specifically focussing on silver. With these fundamentals introduced, plasmonic photocatalysts their formation and mechanism of photocatalytic activity will be explored. The silver/silver halide plasmonic photocatalyst nanoparticles are an integral part of this research project and are discussed in more detail.

Synthesis of nanoparticles requires some form of stabilisation to maintain the size of the particle and prevent agglomeration. In the case of colloidal solutions this is provided by capping agents, or for nanocomposite materials a fine porous host structure restricts the growth and stabilises the nanoparticles. In the case of this research project three matrices were used in the synthesis of silver/silver halide nanocomposites. These were wool fibres, kraft paper fibres and nanostructured calcium silicate. These three materials, their properties and structures are discussed as they are vital in the synthesis of the nanocomposites. Included in this discussion is the synthesis and characterisation of silver halide wool nanocomposites, work previsouly carried out by Dr Fern Kelly and Prof. J. H. Johnston at Victoria University of Wellington. This previous work is the foundation of this research programme. 


\subsection{Silver Halide Photochemistry}

The roles of silver halides in photography, and the readily photoreactive nature of the $\mathrm{Ag}^{+}$ ions, have made silver halides a well researched area. More recently, the focus of research has included applications in imaging, sensors, surface enhanced Raman spectroscopy, photocatalysis, biomedical and antimicrobial applications. ${ }^{1-5}$

The photoreactivity of silver salts and halides has been known for hundreds of years. It was observed that silver nitrate and silver halides would darken on exposure to light. This would form the basis of photography in the years to come. Film processing occurs in a number of steps. Firstly the films containing a silver halide are exposed to light in order to create a latent image, and then processed which involves development, stopping and fixing to form a visible and stable image. The basic process involves silver halide microcrystals suspended in a matrix. For the early photographer this would have been a gelatin matrix fixed to a glass slide to allow exposure without movement of the matrix whilst exposed. After exposure the darkening induced by the light is then enhanced by a developing agent, which further reduces the silver ions to give greater contrast through larger particles with greater absorbencies. The image is then fixed, and finally washed to remove unexposed silver halides that could potentially develop at a later stage. Although photography was widely used, it was not understood until a mechanism was proposed by Gurney and Mott in $1938 .{ }^{6}$

Gurney and Mott proposed that when exposed to light, silver halides absorb photons. This absorption has the required energy to promote an electron from the valence band to the conduction band, leaving a hole. Both of these charge carriers can then migrate throughout the silver halide lattice to charge carrier traps, which are often lattice defects or impurities. Alternatively the charge carriers can undergo recombination. Silver ions migrate towards these defects within the lattice (which contain electrons) and are so reduced. ${ }^{6}$ This process proceeds and a cluster of silver atoms is formed at these sites. In conjunction with this process the holes also migrate through the lattice, and halide ions are oxidised to halogen atoms and are subsequently removed before they can reverse the silver ion reduction. Only a 
few silver atoms are created from initial exposure forming a latent image. These silver cluster latent image centers act as catalysts for the development process through seeding.

Below is a representation of the reaction of silver halides exposed to light and the subsequent combination of the produced silver ion with an electron results in the formation of $\mathrm{Ag}^{06}$.

$$
\begin{gathered}
\mathrm{Ag}^{+} \mathrm{X}^{-}+\mathrm{h} v \rightarrow \mathrm{Ag}^{+}+\mathrm{X}+\mathrm{e}^{-} \\
\mathrm{Ag}^{+}+\mathrm{e}^{-} \rightarrow \mathrm{Ag}^{0}
\end{gathered}
$$

The development in photography uses alkaline conditions along with a reducing agent to reduce silver halide to strings of silver clusters, forming a negative image. This process is then stopped through addition of an acid, and fixed through removal of unreacted silver halide to leave a stable image. The photoreactivity of silver halides has been of great advantage in the development of photography.

Silver chloride, bromide and iodide have all found use in photographic film. Silver bromide has been the most utilized silver halide in photographic and imaging applications as it was found to have very high photosensitivity. This is due to silver bromide grains having a far larger concentration of interstitial silver ions than silver chloride, up to two orders of magnitude larger. ${ }^{3}$ These defect interstitial silver ions promote latent image formation upon light exposure increasing the photosensitivity of the silver bromide grains. Silver iodide on the other hand has been used commonly in photographic emulsion to prevent fogging and increase spectral sensitivity. Silver iodides higher wavelength absorbance $(450 \mathrm{~nm})$ increases the spectral sensitivity. ${ }^{7}$ Silver iodide also has resistance to photolysis, this leads to a decrease in fogging by preventing silver cluster formation away from the exposure areas. The comparatively low photolysis in silver iodide compared to other silver halides is attributed to both to a shorter lifetime of the excited state, and smaller $\mathrm{Ag}-\mathrm{Ag}$ binding energy between interstitial $\mathrm{Ag}^{+}$ions and lattice cations in comparison to the $\mathrm{Ag}-\mathrm{I}$ bonding energy. ${ }^{8}$ The $\mathrm{I}^{-}$ ions are also less mobile through the crystal lattice than $\mathrm{Cl}^{-}$and $\mathrm{Br}^{-}$, this means they are retained in the structure allowing them to re-oxidise $\mathrm{Ag}^{0}$ to $\mathrm{Ag}^{+} .9$ 


\subsection{Silver Halide Nanoparticle Synthesis}

With the advent of nanotechnology the silver halides were not forgotten, and considerable research into their synthesis and characterisation has been, and continues to be, done. Synthesis of silver halide nanoparticles is relatively straight forward and has been achieved through reverse micelle, ionic liquids and aqueous methods. ${ }^{4,10-15}$

Reverse micelle (RM) synthesis is very popular due to the extremely fine, monodisperse particles it can produce $(<10 \mathrm{~nm}) .{ }^{11}$ The RM synthesis methods involve water in oil emulsions to control the growth of the nanoparticles. These emulsions are composed of water droplets containing the reactants, which are stabilized by surfactant molecules within an oil solvent. It is generally believed that particles are grown in these RM systems through the association and dissociation of the water pools in the emulsion. The water pools are used as nanoreactors, containing limited reactant species allowing controlled growth. ${ }^{16}$ An example of a common reverse micelle synthesis method for silver halides would be as follows: a water in oil emulsion would be made containing $\mathrm{AgNO}_{3}$, stabilized by a surfactant such as dioctyl sodium sulfosuccinate (AOT) or Tergitol (NP-6) which has a hydrophilic head group and a large hydrophobic tail allowing it to form reverse micelles containing aqueous pools of $\mathrm{AgNO}_{3}$, within $\mathrm{n}$-decanol or $\mathrm{n}$-heptane. A second, similar emulsion is also made with $\mathrm{NaCl}$ instead of $\mathrm{AgNO}_{3}$ within the water pool. Upon mixing of the two RM emulsions, exchange of reactants between water droplets allows formation and growth of $\mathrm{AgX}$ nanoparticles. ${ }^{10,13}$

Aqueous nanoparticle synthesis is desirable because of the relatively low waste products and the ease of synthesis. Aqueous synthesis often involves solutions of reactants being prepared in an aqueous environment along with a stabilizing and capping agent in order to control growth to obtain the small particle dimensions required. These methods are similar to the traditional way of making noble metal nanoparticles through aqueous synthesis, where $\mathrm{AgNO}_{3}$ is used in aqueous solution with poly (vinyl pyrrolidone) (PVP), which acts as both the reductant and stabiliser. Under heat, $\mathrm{AgNO}_{3}$ is reduced to $\mathrm{Ag}^{0}$ by PVP, and the resultant silver nanoparticle is subsequently capped by PVP thus limiting its size. For silver halide nanoparticle synthesis instead of using reducing conditions, $\mathrm{HCl}$ or $\mathrm{NaCl}$ is added to the 
solutions of $\mathrm{AgNO}_{3}$, initiating the precipitation of silver halide nanoparticles, which are then capped by the PVP. ${ }^{4}$ These silver halide nanoparticles can then be used as a base to synthesise silver/silver halide nanoparticles. These nanoparticles are formed using the photoactive nature of silver halides to form silver. The silver halide nanoparticles are exposed to light to induce formation of silver nanodomains on the surface of the silver halide nanoparticle. These silver/silver halide nanoparticles display photocatalytic activity, and their structure and synthesis is described in further detail in the following sections.

\subsection{Metal Nanoparticles and Surface Plasmon Resonance}

Silver has found use in applications that exploit its inherent worth; applications including jewellery, decoration and currency, that are suitable due to the bulk metal being relatively unreactive and having a pleasant bulk colour. This bulk appearance can be modified when particles of the metal are on the nanometer length scale, with one dimension $<100 \mathrm{~nm}$. Once down at this length scale the observed colour of the nanoparticle is dependent on both its size and shape, due to variations of the visible absorptions of the particle. It is a property that has been observed, although unwittingly, since the Middle Ages. The ruby reds and deep purples of stained glass windows and the Lycurgus cup are two examples of colloidal metals used as colourants. The red of the stained glass is due to spherical gold nanoparticles forming as a result of gold added by alchemists during the glass production. The Lycurgus cup is a mixture of gold, silver and copper nanoparticles. The gold is mostly responsible for the red colour observed in transmission, silver for the green when in reflectance. ${ }^{17}$
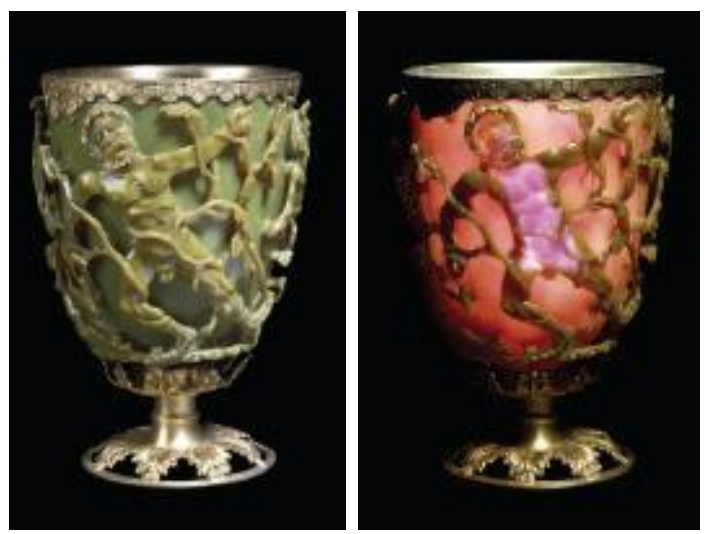

Figure 1 Lycurgus cup in reflected (a) and transmitted (b) light. ${ }^{17}$ 
The source of the rich colouration was unknown until 1856 when Michael Faraday suggested that the colour observed in stained glass windows was a result of finely divided metal particles. ${ }^{18}$ A theoretical explanation to Faraday's proposed reason for the colouration had to wait until 1908. This was provided by Mie, and it is a phenomenon now called surface plasmon resonance (SPR). ${ }^{19} \mathrm{SPR}$ is caused by the oscillation of conduction band electrons induced by the electromagnetic fields of light. ${ }^{20}$ In conductive materials such as metals, the conducting electrons are delocalised giving rise to constant movement of electron density. The quantised electron oscillations are called plasmons, under the influence of electromagnetic fields of light the plasmons resonate along a longitudinal wave as a harmonic. ${ }^{21}$

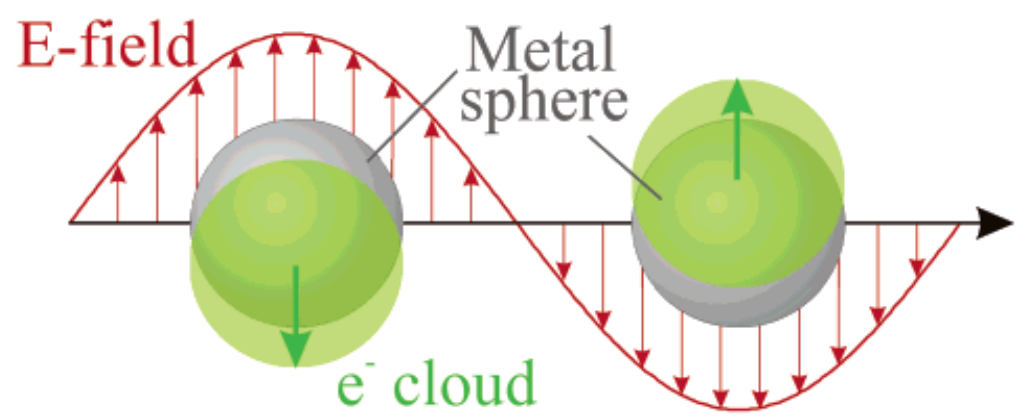

Figure 2 Plasmon oscillation in metal nanospheres. ${ }^{22}$

In order for photons to couple with the plasmon oscillations the incident energy of the photon must be comparable to that of the plasmon. In a bulk metal the plasmons are longitudinal and the incident photons are transverse, and this prevents coupling without changing the incident beam to travel parallel with the plasmon oscillation. In scenarios with nanoparticulate conductive materials, the electric field induced by the plasmon is affected by the surface. This essentially gives the plasmon resonance both transverse and longitudinal wave components, allowing the coupling of the photons with plasmons without the need for a grating or prism to adjust the incident light.

The wavelength at which this coupling occurs is known as the surface plasmon resonance band (SPRB), the intensity and wavelength at which this occurs is dependent on size and shape of the nanoparticle and the dielectric functions of the surrounding medium. ${ }^{20}$ The size 
of the particle affects the wavelength position of the surface plasmon resonance band. This is due to the wavelength being dependent on the radius of the particle. An increase in particle size leads to an increase in the wavelength of the surface plasmon resonance band. Particle size decreases however can lead to a decrease in intensity of the resonance band and an accompanying broadening. This broadening is due to an increase of surface scattering of conduction electrons, as well as having a smaller resonance because of the decreased size.

The shape dependence of the SPRB arises from the lack of interaction of the incident photons with surface plasmons without a transverse wave component. This makes spherical shape and curvature very important in the SPRB presence. The spherical particles display a singular plasmon resonance band, due to the dipole induced by the interaction of the electromagnetic radiation oscillating in the phase of the incoming light. In spherical particles only one interaction is possible, and so only one band is seen. In for example nanorods, where there is both a transverse and longitudinal interaction, both can be seen, giving rise to two SPRB's arising from the two different dipoles produced through plasmon and light interaction. ${ }^{20}$ (Figure 3) In the case of nanorods the second SPRB absorption is dependent on the aspect ratio of the rod, allowing different colouration to be observed as can seen in Figure 4 below. Both transverse and longitudinal SPRB's are observed, the longitudinal SPRB arises and red shifts as aspect ratio increases. Whilst the transverse SPRB decreases in intensity with increases in aspect ratio, both SPRB influence the observed colouration of the nanorods.

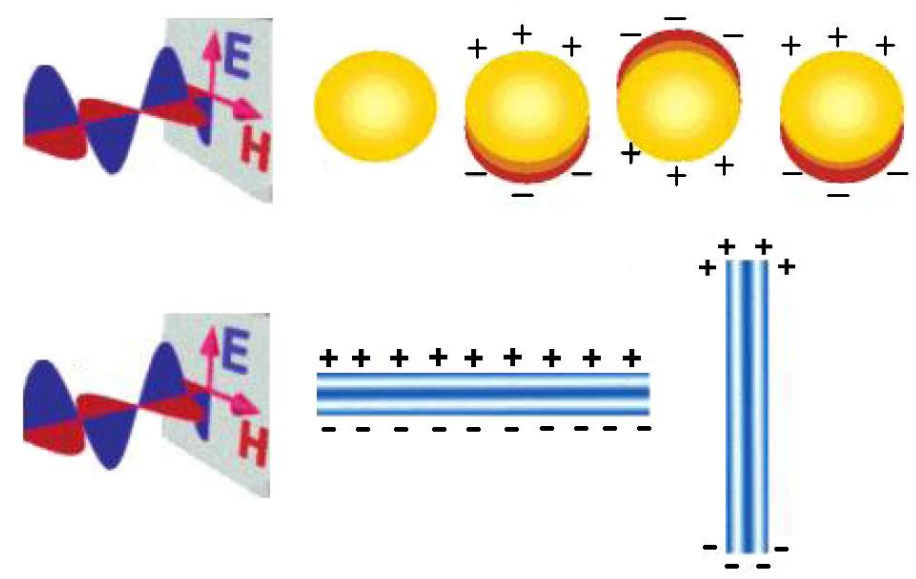

Figure 3 Interaction of the electromagnetic field of incoming light with a) spherical nanoparticles, and b) nanorods. ${ }^{20}$ 


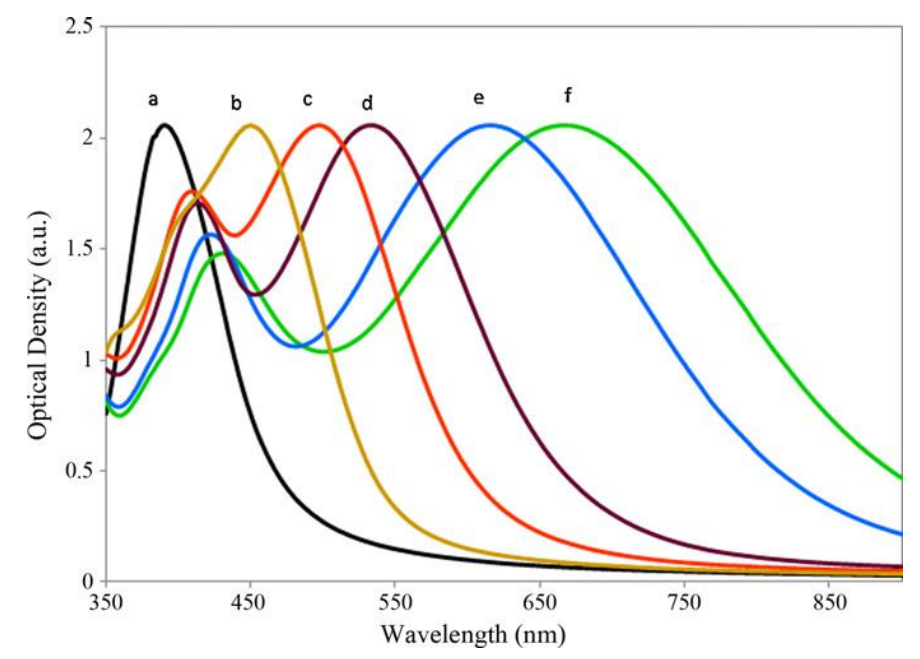

Figure 4 Absorption profile of Ag nanorods with increasing aspect ratios ${ }^{23}$

The difference in dielectric constants of the surface and surrounding medium also has a distinct effect on the SPRB. The change in dielectric constant has an ability to alter the refraction of incoming photons, giving rise to a change in energy and wavelength. The change from the bulk plasmon wavelength to that of the resonant wavelength of a nanoparticle is a function of the dielectric constant of the medium and metal. Described by the formula: ${ }^{24}$

$$
\lambda=\lambda_{p} \sqrt{ }\left(\varepsilon_{\infty}+2 \varepsilon_{m}\right)
$$

where $\lambda$ is the resonant wavelength, $\lambda_{p}$ is the bulk Plasmon wavelength, $\varepsilon_{\infty}$ is the dielectric constant of the metal and $\varepsilon_{m}$ is the dielectric constant of the medium. This makes the dielectric constant important in the position and intensity of the SPRB. ${ }^{24}$ Through introduction of coatings that change the dielectric constant, it is possible to create a wide range of SPRB's to allow a greater variety of tuning of the plasmon resonance absorption. For silver this can mean a large spread of tunable wavelengths, however increases in dielectric constant tend to induce a red shift. 


\subsection{Silver Nanoparticle Surface Plasmon Resonance}

Surface plasmon resonance has been discussed in a general sense in the previous section and silver nanoparticles and the SPR that they exhibit will be more specifically explored. Silver nanoparticles have interesting light scattering and absorption properties for surface enhanced Raman spectroscopy and solar absorption coatings. ${ }^{25,26}$ Surface plasmon resonance plays a vital role in the observed optical properties of metal nanoparticles (as mentioned previously), and silver is no exception. Silver exhibits shifts in absorbance depending on the size and shape of the nanoparticle. ${ }^{27}$ For example a spherical silver nanoparticle of $50 \mathrm{~nm}$ absorbs strongly in the blue region of visible light, taking this colour away from the reflected light due to its strong absorption producing the observed yellow colour when viewing the silver nanoparticles. ${ }^{28}$ A small increase in particle size gives a red shift of the light absorbed, and it is this strong absorption that gives the nanoparticles their bright observed colouration.

Increasing the size of spherical silver nanoparticles red shifts the wavelength of light absorbed. As particle sizes approach $90 \mathrm{~nm}$ the peak absorbance is seen to increase to $490 \mathrm{~nm}$ wavelength, as opposed to the $420 \mathrm{~nm}$ absorption of the $50 \mathrm{~nm}$ size spheres. The effect this has on the colouration of the particles to the eye is a shift from the yellow seen in the smaller sized silver nanoparticles to a red with the larger particle size. ${ }^{27,28}$ The colour reflected also exhibits strong shape dependence as mentioned previously. The primary shapes seen for silver nanoparticles are spheres, triangles, platelets, decahedrons and pentagons. The wavelengths of different shape absorption have been correlated as shown below. (Figure 5) The more unsymmetrical a shape the more SPRBs there are, due to an increase in different transverse and longitudinal interactions. Increasing aspect ratios and shape size also allows tuning of colouration to a further extent in similar ways to that of spherical nanoparticles. ${ }^{28,29}$ 


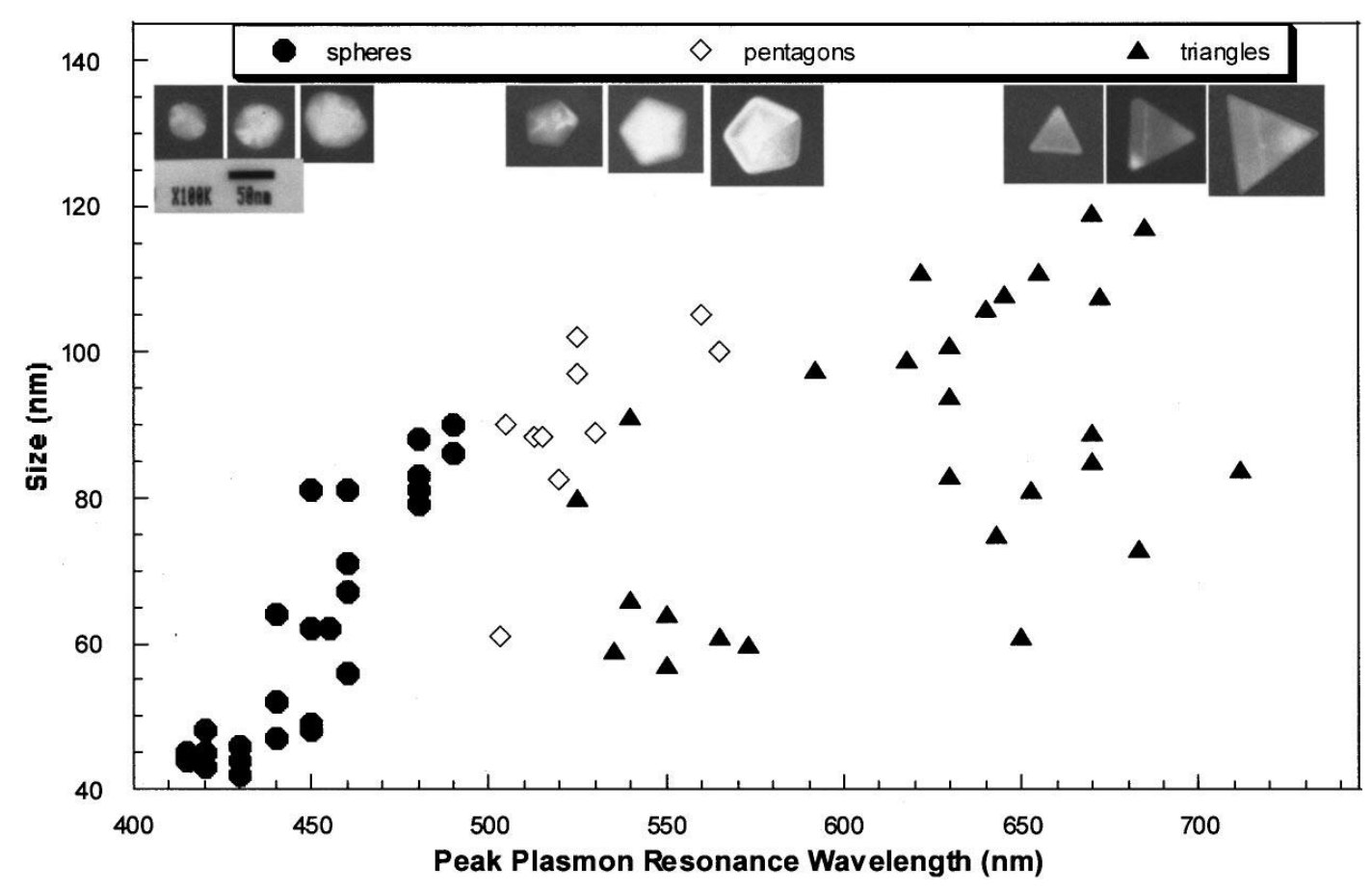

Figure 5 Size and shape of silver nanoparticles correlated with absorbed wavelength of light. ${ }^{28}$

\subsection{Silver/Silver Halide Plasmonic Photocatalysts}

One of the most recent areas of research involving silver halides and silver halide nanoparticles has been in the synthesis of plasmonic photocatalysts. There is a huge area of interest in relation to photocatalysts, in particular properties such as photoelectrochemical solar energy conversion. The photocatalytic materials could be potentially applied in the fields of water splitting, self-cleaning surfaces, and decomposition of organic pollutants. ${ }^{30-33}$ These applications have lead to a considerable amount of study in the photocatalyst area. The main approach to creating photocatalysts for these applications has been focused on improving the photocatalytic activities of titania $\left(\mathrm{TiO}_{2}\right)$. This is because titania, in particular the anatase crystal structure, is a cheap non-toxic material that shows photocatalytic activity. However the bandgap of $\mathrm{TiO}_{2}$ is large $(3.2 \mathrm{eV})$ and only higher energy UV light $(\lambda<390 \mathrm{~nm})$ can generate the electron-hole pairs required for photocatalysis. This very much restricts the efficiency of $\mathrm{TiO}_{2}$ in photocatalytic applications. ${ }^{31,34}$ In order for commercial applications of photocatalysis to be viable, higher efficiency systems that utilise visible light must be sought. Recently silver/silver halide nanoparticles have been found to be active in visible light photocatalysis and are part of the new family of plasmonic photocatalysts. 
The first $\mathrm{Ag} / \mathrm{AgCl}$ plasmonic photocatalysts were proposed very recently, ${ }^{35,36}$ and since then they have become a very rapidly growing area of research. ${ }^{37-43}$ Plasmonic photocatalyst systems differ from traditional photocatalysts through exploiting the SPR phenomenon. The localised SPR of noble metal particles in contact with a semiconductor produce plasmonic photocatalysts. The interaction of incident photons with the noble metal nanoparticle free electrons through SPR produces strong visible light absorbance as previously mentioned. The SPR allows excitation of the conduction band electrons when there is resonance between the conduction band electrons and the oscillating electric field of the incident photon. This means an absorption in the UV/Vis and NIR is possible if the SPR of the noble metal particle falls within these wavelengths. This SPR can be used to improve absorption of photocatalysts.

Plasmonic photocatalysts have been synthesised in a number of ways and have been seen to be efficient stable photocatalysts even under visible light. Plasmonic photocatalysts made through noble metal nanoparticles embedded or immobilised in semiconductors have been made to improve photocatalysts such as titania. These noble metal particle photocatalysts have been found to have high absorption coefficients in UV-Vis-NIR due to the SPR of the metal particles, and were resistant to degradation caused by irradiation. ${ }^{36,41}$ These types of plasmonic photocatalyst are synthesis intensive, involving multiple steps leading to uncertainties and plasmonic nanoparticle-semiconductor interfaces that are not well controlled. An alternative approach to synthesis is through hybrid semiconductor nanoparticles. In this approach a particle of semiconducting photocatalyst can be partially converted to plasmonic metal nanoparticles. This results in a hybrid semiconductor photocatalyst particle with plasmonic photocatalyst properties. Silver halides are a good example of such photocatalysts. The semiconducting nature of silver halides provides an excellent basis for such particles, as $\mathrm{Ag}^{+}$from the $\mathrm{AgX}$ can be partially converted to $\mathrm{Ag}^{0}$. This creates discrete $\mathrm{Ag}^{0}$ nanoparticle domains on the surface of the semiconducting $\mathrm{AgX}$ nanoparticle. This leads to a dual interaction with light, the $\mathrm{Ag}^{0}$ nanodomains exhibit SPR increasing the visible region absorption of the nanoparticles. Secondly the $\mathrm{Ag}^{0}$ nanodomains also allow charge separation within the nanoparticle using the metal and semiconductor domains which will be discussed further. 
Silver/silver halide plasmonic photocatalysts have been synthesised via two approaches. One approach is through an ion-exchange reaction between aqueous solutions of $\mathrm{Ag}_{2} \mathrm{MoO}_{4}$ and $\mathrm{HCl}$ (or $\mathrm{HBr}$ ). This multistep method of $\mathrm{Ag} / \mathrm{AgCl}(\mathrm{AgBr})$ particle synthesis has been used to achieve particles of a variety of shapes. Microwave assisted hydrothermal synthesis of the $\mathrm{Ag}_{2} \mathrm{MoO}_{4}$ is used to create a template for the $\mathrm{Ag} / \mathrm{AgCl}(\mathrm{AgBr})$ particles. This was achieved by preparing solutions of $\mathrm{AgNO}_{3}$ and $\mathrm{Na}_{2} \mathrm{MoO}_{4}$, combining them to created a mixed solution. The $\mathrm{pH}$ was then adjusted to 8.0 by adding dilute $\mathrm{NaOH}$ solution. This mixed solution was then heated at $180{ }^{\circ} \mathrm{C}$ for $1 \mathrm{~h}$ under microwave irradiation, precipitating $\mathrm{Ag}_{2} \mathrm{MoO}_{4}{ }^{44}$ The sacrificial $\mathrm{Ag}_{2} \mathrm{MoO}_{4}$ templates are then dissolved in excess acid $(\mathrm{HCl}$ or $\mathrm{HBr})$ where the ion exchange reaction occurs. ${ }^{44}$

$$
\mathrm{Ag}_{2} \mathrm{MoO}_{4}+2 \mathrm{HCl} \rightarrow 2 \mathrm{AgCl}+\mathrm{MoO}_{3}+\mathrm{H}_{2} \mathrm{O}
$$

The AgX products are then collected and irradiated with a Xe arc lamp to partially reduce some $\mathrm{Ag}^{+}$to $\mathrm{Ag}^{0}$. This creates $\mathrm{Ag} / \mathrm{AgX}$ particles where $\mathrm{AgX}$ nanoparticles are covered with Ag nanodomains.

The second synthesis method for $\mathrm{Ag} / \mathrm{AgX}$ particle synthesis is a straight forward direct reaction of $\mathrm{AgNO}_{3}$ and $\mathrm{HCl}$ to form $\mathrm{AgCl}$, followed by $\mathrm{UV}$ irradiation, which converts $\mathrm{Ag}^{+}$ ions to $\mathrm{Ag}^{0}$ nanodomains on the surface of the $\mathrm{AgCl}$ particles. Choi et al. developed a method of $\mathrm{Ag} / \mathrm{AgCl}$ nanoparticle synthesis for use as plasmonic photocatalysts via this route. ${ }^{45}$ Silver chloride nanoparticles were synthesised through direct reaction of $\mathrm{AgNO}_{3}$ and $\mathrm{HCl}$ in aqueous solution using a PVA dispersing agent. Silver chloride nanoparticles were collected and irradiated with a $\mathrm{Xe}$ arc lamp to create the $\mathrm{Ag} / \mathrm{AgCl}$ particles, as shown in the schematic below. A method very similar to this was utilised for the creation of the $\mathrm{Ag} / \mathrm{AgX}$ particles used in this research project. 


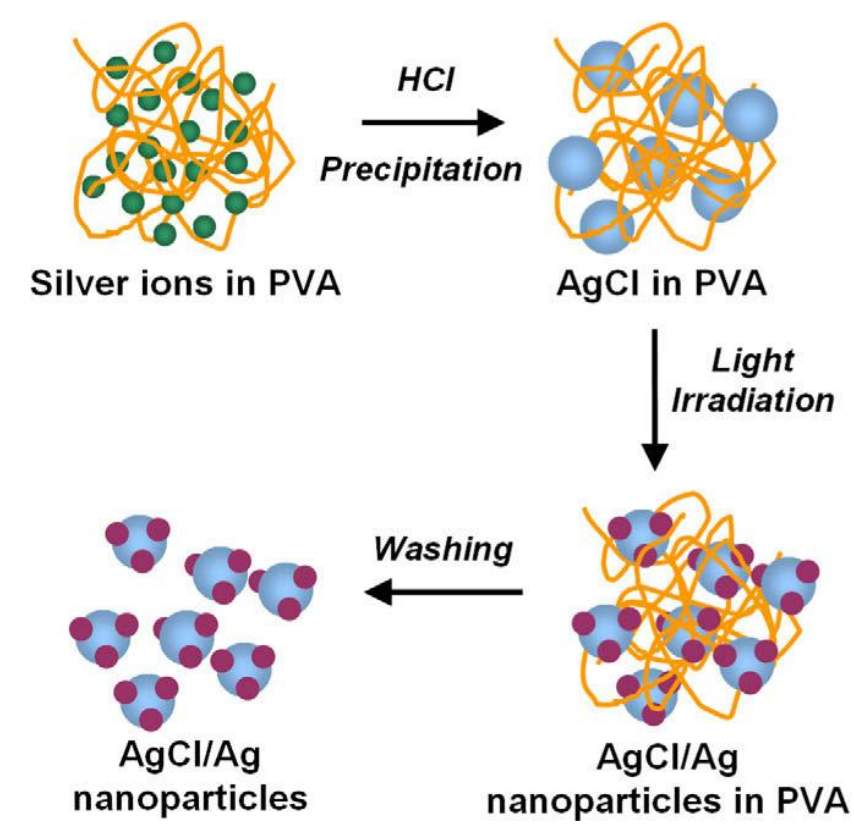

Figure 6 Schematic of the synthesis of $\mathrm{Ag} / \mathrm{AgCl}$ nanoparticles ${ }^{45}$

The silver/silver chloride nanoparticles display visible light activity, unlike plain silver chloride nanoparticles. This is generally attributed to the SPR of the silver nanodomains on the surface of the silver halide nanoparticle. Under irradiation the silver halide particles undergo a self-sensitisation producing the surface silver nanoparticles but preventing total reduction to $\mathrm{Ag}^{0}{ }^{46,47}$ This is believed to be due to the two regions of the particles. Once sufficient surface $\mathrm{Ag}^{0}$ particles have been formed, when incident photons are absorbed and interact with the $\mathrm{Ag}^{0}$ surface plasmon resonance an electron-hole pair is formed. The local electromagnetic field of the particle then assists in the separation of the electron-hole pair. The interface between the $\mathrm{AgCl}$ and $\mathrm{Ag}$ domains has partial negative polarisation as it is depleted in silver cations from the self-sensitisation. ${ }^{35,48}$ The areas away from this interface have a partial positive polarisation, further assisting in electron-hole separation. This effectively allows the electrons and holes to flow to separate domains. This separation of charge has made the plasmonic photocatalyst particles particularly photostable under irradiation. ${ }^{32,44}$ This charge separation has also allowed for these plasmonic photocatalytic particles to be used to degrade dyes such as Methylene Blue (MB) and Methyl Orange (MO) using visible light. ${ }^{32,35,39,45}$ 
The dyes are used to simulate organic contaminants, and previous research has demonstrated that silver/silver halide nanoparticles are effective for applications involving organic contaminant degradation. ${ }^{32,35,39,43,45,48}$ The literature suggests the mechanism of degradation of these dyes is through radical formation. Under light irradiation the $\mathrm{Ag} / \mathrm{AgCl}$ nanoparticles absorb light; visible light absorption is assisted through SPR, creating an electron-hole pair. This electron-hole pair then separates and migrates to the metallic and semiconductor regions of the particle respectively. Then both the electron and hole are intiate radical formation. The silver/silver chloride nanoparticles' distinct regions lead to radical formation in two different ways. When in an aqueous environment, the $\mathrm{Ag}^{0}$ region will form super oxide and other radical oxygen species that degrade the organic dye by the reduction of dissolved oxygen in solution. In a similar manner the silver chloride region also degrades the dye through radical decomposition. The hole in the $\mathrm{AgCl}$ region forms $\mathrm{Cl}^{0}$, a radical species and good electron acceptor that breaks down organic compounds when in contact with them, further returning $\mathrm{Cl}^{0}$ to $\mathrm{Cl}^{-32,35,45}$

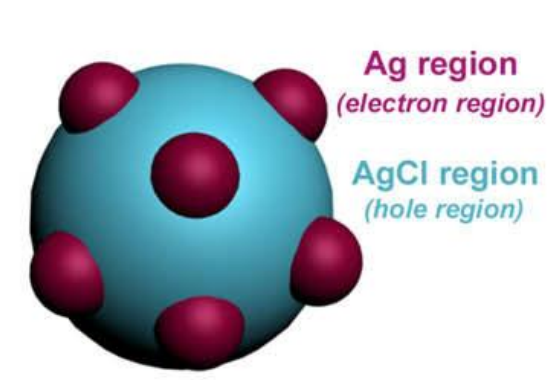

Separated electron-hole region
Under visible irradiation,

$$
h v \longrightarrow \mathrm{e}^{-} \text {(electron) }+\mathrm{h}^{+} \text {(hole) }
$$

\section{In AgCl region,}

$\mathrm{Cl}^{-}+\mathrm{h}^{+} \longrightarrow \mathrm{Cl}^{0}$

$\mathrm{Cl}^{0}+$ organic compound $\longrightarrow \mathrm{CO}_{2}+\mathrm{H}_{2} \mathrm{O}+\mathrm{Cl}^{-}$

In Ag region,

$\mathrm{O}_{2}+\mathrm{e}^{-} \longrightarrow \mathrm{O}_{2}^{-}$

Figure 7 Scheme showing the structure of $\mathrm{Ag} / \mathrm{AgCl}$ nanoparticle and radical formation ${ }^{45}$

These plasmonic photocatalyst particles have been synthesised using a variety of silver halides including $\mathrm{AgCl}, \mathrm{AgBr}$ and $\mathrm{AgI} .{ }^{48,49}$ Not only just the silver halides by themselves but combinations halides such as $\mathrm{AgCl}, \mathrm{Br}$ and $\mathrm{AgBr}, \mathrm{I}$ have shown activity. ${ }^{39},{ }^{43} \mathrm{Ag}_{3} \mathrm{PO}_{4}$ and $\mathrm{AgO}$, have shown to display similar photocatalytic properties. ${ }^{40,42,50}$ 
The main aim of this research project is to synthesise and characterise these silver/silver halide nanoparticles and nanocomposites using a porous substrate. $\mathrm{AgCl}, \mathrm{AgBr}$ and $\mathrm{AgI}$ will be used to produce silver/silver halide nanoparticles. Silver/silver halide nanocomposites will also be produced with natural fibres such as wool and kraft paper, as well as nanostructured calcium silicates. These nanocomposites will be characterised in a similar manner to the nanoparticles in order to determine if $\mathrm{Ag} / \mathrm{AgX}$ nanoparticles have been successfully incorporated in the substrates. Finally as these $\mathrm{Ag} / \mathrm{AgX}$ nanoparticles have been shown to be plasmonic photocatalysts, the photocatalytic properties of these nanocomposites will be evaluated.

\subsection{Structure of Wool}

Wool fibres have been selected as a substrate for the synthesis of silver/silver halide nanocomposites. This was partly due to previous work carried out by the research group in related nanocomposites, as well as its suitability as a matrix. The wool fibre is porous making allowing the formation of $\mathrm{AgCl}$ nanoparticles within the fibre. This makes the structure and properties of wool of interest in this research project. The structure of wool is important in determining its properties. Wool is made up of proteins. Proteins are natural polymers formed from polymerisation of $\alpha$-amino acids. Merino wool and Crossbred wool, as well as hair, are fibres made up by the $\alpha$-keratin class of proteins. The elasticity, flexibility and moisture absorbent properties of wool are a result of this $\alpha$-keratin structure. The $\alpha$-keratin protein is $\alpha$ helix shaped, this helix shape allows the fibre to act similar to a spring allowing compression, stretching and bending of the fibre. The $\alpha$-helix shape is a product of the amino acid interactions, through amino acid residue bonding and intramolecular hydrogen bonding. ${ }^{51}$ In terms of amino acids, many different residues are found within the keratin protein structure. One important residue is cystine which, combined with cysteine, give the high sulfur content of keratin. ${ }^{52}$ Cystine residues contain disulfide linkages which play an important role in the properties and characteristics of the wool. 


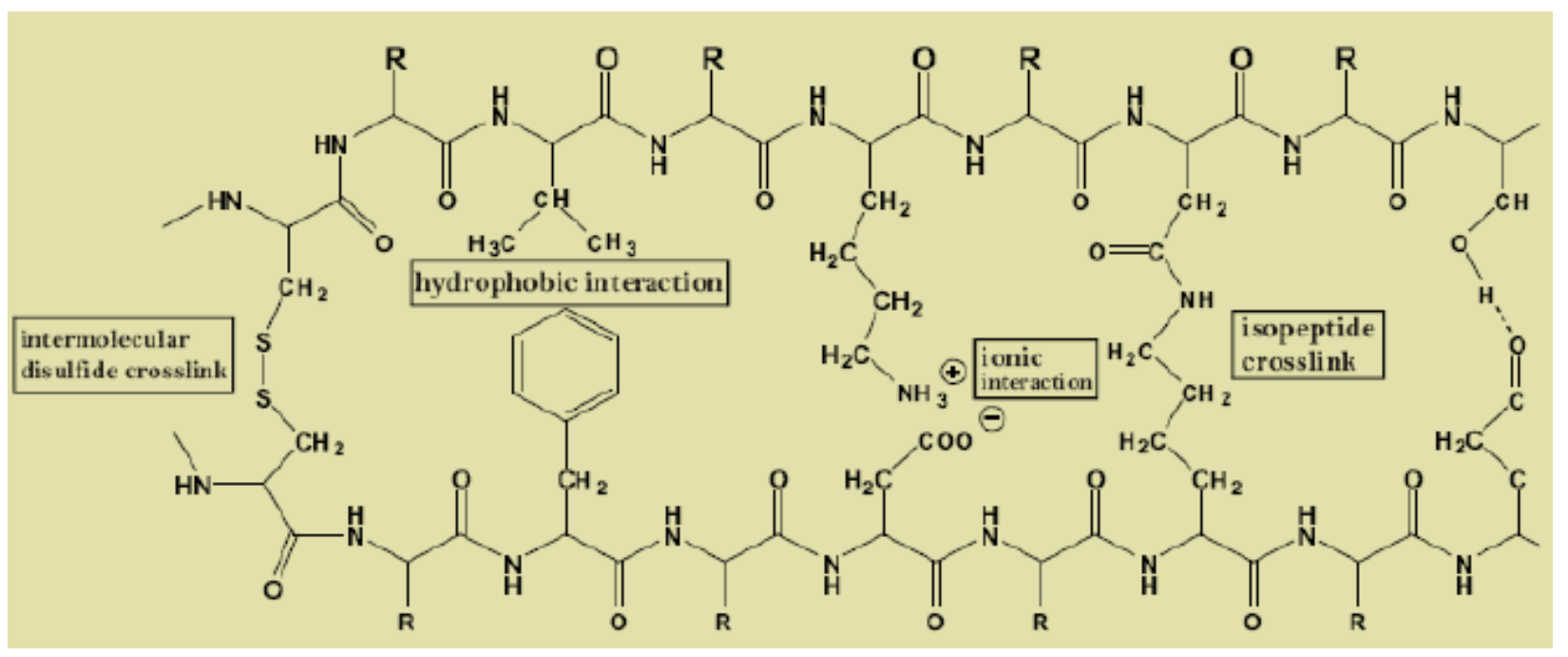

Figure 8 Bonds liking adjacent polypeptide chains in wool $\alpha$-keratin fibres. ${ }^{51}$

The structure of the fibre is complex, with an outer layer of cuticle shells surrounding the central cortex. These outermost cuticle cells overlap in a fashion similar to roofing tiles, pointing out toward the fibre tip. This creates a large amount of friction when the fibre is forced in the directionality opposite to that of the cuticle growth. ${ }^{53}$ Cuticle size is a property that defines different fibres; even within the $\alpha$-keratin type fibres, differences in cuticles can be easily discernable. ${ }^{54,55}$ The outermost layer of the cuticle is what provides protection and the strong stability of the fibre; this is known as the epicuticle. It is a membrane which is resistant to alkali, acidic and oxidising agents; although mostly consisting of proteins, the epicuticle contains a substantial lipid component. ${ }^{56,57}$ The lipid bound covalently to the fibre surface imparts the hydrophobic characteristic to the wool fibre surface. ${ }^{58,59}$ This has a negative impact in terms of wool processing with the hydrophobic nature making the dyeing process difficult. Often in processing, surface treatments are implemented to modify the surface properties of the wool fibres, in order to remove the fatty acid layer or minimise the hydrophobic nature to increase dye uptake. ${ }^{56,60,61}$ 


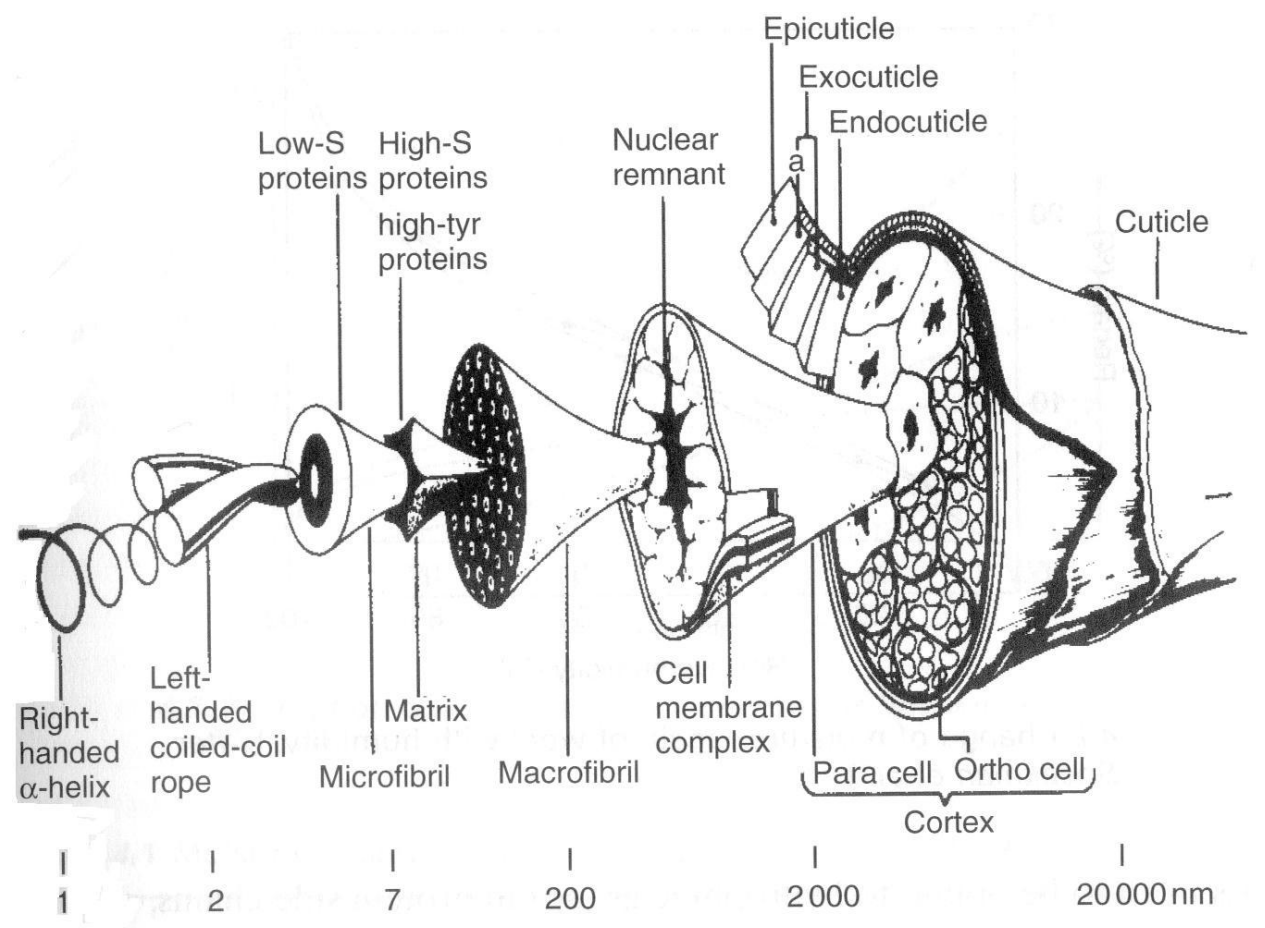

Figure 9 Structure of the $\alpha$-keratin fibre. ${ }^{52}$

Beneath the cuticle shell lies the cortex and cortical cells which make up $90 \%$ of the fibre. There are two types of cortical cell, orthocortical and paracortical. ${ }^{62}$ In finer fibres, these two types of cells form in two distinct segments. The two cell types have slightly different chemical composition, this makes the cells expand differently when they absorb moisture. This differential expansion makes the fibre bend, inducing the crimp observed in wool. ${ }^{63}$ In coarser fibres, as the fibre diameter increases the percentage of paracortical cells increases. In the case of crossbred fibres there sometimes exists an outer edge of paracortical cells surrounding a centre of orthocortical cells, resulting in less crimp. Crimp allows the fibres to be elastic, easily bent and twisted without damage, it also enhances the trapping of air between fibres providing insulating air pockets.

Inside the cortical cells are long filaments of macrofibrils, these are $\sim 0.5$ micron diameter bundles, comprising of even finer microfibrils. The microfibrils are surrounded by a matrix region of high cystine content. ${ }^{64}$ This region is also responsible for wool's fire-resistance and anti-static properties. The microfibrils contain pairs of twisted rope like coils, comprising of the $\alpha$-helical protein chains. ${ }^{64}$ This helical protein structure as mentioned previously gives 
wool its flexibility, elasticity and resilience through its hydrogen bonding, disulfide linkages and intramolecular interactions. These properties allow the fabric to keeps its shape, limiting wrinkling.

The medulla is a series of vaculated cells that run along the axis of some coarser large diameter $\alpha$-keratin fibres. They are highly alkali resistant, providing stability to the keratin fibres. The medulla can be classified as continuous, discontinuous or fragmented. ${ }^{65}$ The medulla is essentially empty air filled space along the length of the fibre. Medullated fibres are often considered contaminants as they do not uptake dyes in a uniform manner and often leave white fibres in the final wool product. ${ }^{66}$ Finer wool is often non-medullated.
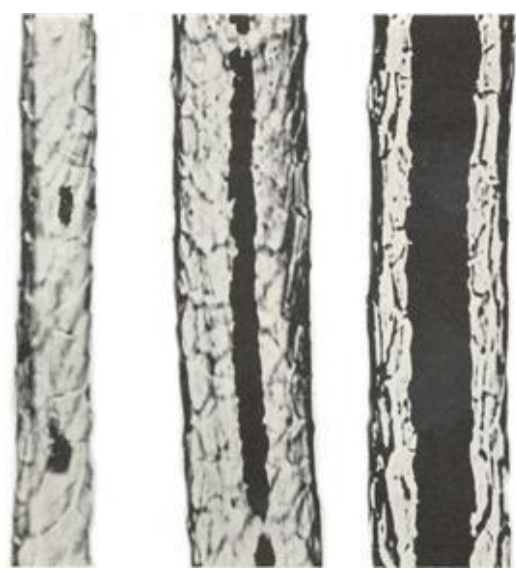

Figure 10 Types of medulla that can occur in wool fibres. a) Fragmental, b) interrupted and c) continuous. ${ }^{1}$

\subsection{Silver Halide Wool Nanocomposites}

Silver halide wool nanocomposites have been developed previously by Prof. J. H. Johnston and $\mathrm{Dr}$ Kelly of Victoria University of Wellington. ${ }^{67}$ These silver halide wool nanocomposites were developed as colourants for merino and crossbred wool, as well as antimicrobial agents. Kelly produced these composites by doping merino and crossbred wool with halide ions at acidic $\mathrm{pH}$. Following the pre-treatment of doping the wool fibre and $\mathrm{pH}$ adjustment, silver nitrate was added forming the silver halide wool nanocomposite. ${ }^{67}$ These silver halide wool nanocomposites showed some interesting photoactivity. 
The $\mathrm{AgCl}$ and $\mathrm{AgBr}$ wool nanocomposites immediately after synthesis were white in colour. However after exposure to light they underwent a colour change from white to purple. Silver chloride and silver bromide are themselves white in colour, and so the white nanocomposite is to be expected. The known photoactivity of the silver halides also meant that having a colour change due to light irradiation was not unexpected. Kelly then comprehensively characterised these nanocomposites in order to further explore their photoactivity. The absorption of light of the $\mathrm{AgCl}$ and $\mathrm{AgBr}$ wool nanocomposites was monitored by UV/Vis diffuse reflectance spectroscopy over the period of colour development. It was seen that a distinct and broad visible absorbance developed over the light exposure time. It was suggested that this behaviour may be due to formation of silver nanoparticles within the $\mathrm{AgCl}$ medium with the shift in SPRB due to the dielectric constant of $\mathrm{AgCl} .{ }^{67}$

Scanning electron microscopy (SEM) of the silver halide wool nanocomposites was also carried out to confirm the presence of the silver halide species within the wool matrix. SEM images of the silver halide wool composites showed nanoparticles within the wool fibres of varying sizes. ${ }^{67}$ Agglomeration was largely observed, however SEM appeared to show a relatively smooth surface to the composite wool fibres, suggesting that the nanoparticles were all formed within the fibre itself and not loosely bound to the surface. Energy Dispersive Xray Spectroscopy (EDS) from both SEM and Scanning Transmission Electron Microscopy (STEM) confirmed that the nanoparticle species observed were silver and the respective halide depending on the sample. ${ }^{67}$ Transmission Electron Microscopy (TEM) was used to further characterise the silver halide nanoparticles within the wool fibres. TEM images showed a variety of silver halide particles of different sizes and shapes. A mixture of both spherical and non-spherical particles was observed. High resolution TEM of the nonspherical nanoparticles showed twinning planes within the particles. ${ }^{67}$ As silver halides have cubic crystal structures it is possible for them to create a variety of shaped nanoparticles. These range from triangular platelets to hexagonal and octahedral shapes as well as cuboids. This interesting mixture of shapes and sizes tends toward particle growth being a function of pore size within the wool fibre matrix. It was also seen that the silver halide nanoparticles were formed beneath the surface of the fibre and were not distributed throughout the entire 
cross section. The method of nanoparticle formation was postulated to be through precipitation and then size control imposed by the matrix. ${ }^{67}$

To further confirm the silver halide presence within the wool fibres synchrotron X-ray diffraction was carried out on the silver halide wool nanocomposites. The X-ray diffraction of the nanocomposites confirmed the presence of the silver halides within the wool fibre matrix. More interestingly however there were indications of crystalline Ag being present within the samples with a very small Ag 111 peak visible. An in situ experiment within the synchrotron showed the formation of an $\mathrm{Ag} 111$ peak during irradiation as the purple colour developed from a white sample kept in the dark. ${ }^{67}$ No colour change was observed in the blank wool sample exposed to synchrotron radiation.

These silver halide composites that were created by Kelly and Johnston have UV/Vis spectra and XRD patterns that resemble that of the $\mathrm{Ag} / \mathrm{AgX}$ plasmonic photocatalysts. The hybrid $\mathrm{Ag} / \mathrm{AgX}$ nanoparticles may in fact be what are formed in situ within the wool fibre matrix. One aim research project was to confirm whether these silver halide wool nanocomposite, are in fact silver/silver halide nanocomposites. This will be carried out by recreating and characterising the silver halide wool nanocomposites, for comparison with characterised silver/silver halide nanoparticles. With this in mind it should be possible to create further nanocomposites of a similar nature. These nanocomposites may also have photocatalytic properties and may be of use as solid matrix stabilised photocatalysts as well as being antimicrobial agents. In this project materials that were explored as a matrix in a similar manner as to the way the wool is in these nanocomposites, were kraft paper fibres and nanostructured calcium silicate.

\subsection{Bleached and Unbleached Kraft Paper Fibres}

Kraft paper is one of the strongest papers produced by chemical pulping and has a characteristically high tensile strength. This strength is reflected in the applications that kraft paper is often used for, notably, packaging, box lining, and particleboard and sandpaper 
bases. These are all applications that require the paper to be high strength and durable. In particular, unbleached Kraft paper is used in packaging applications due to its characteristic brown colour. This colour is due to the presence of remaining lignin within the pulp. ${ }^{68}$ Bleached kraft is white in colour due to removal of lignin components through a bleaching process. This is required for high quality pulp used in paper.

Both bleached and unbleached kraft fibres are produced from wood matter. Most commonly used are softwoods, however hardwoods and other sources can also be used. Wood fibres are primarily carbohydrate in nature. A particularly abundant and important carbohydrate component is cellulose, the main component of paper fibres. However non-cellulose contributions such as hemicellulose and lignin play a role in the structure and properties of kraft paper, as it is made from the pulp of woody plant matter which also contains these components. Cellulose is a polymer made up of repeating carbohydrate $\beta-(1 \rightarrow 4)$-linked glucose units. ${ }^{68,69}$ The general chemical formula for cellulose polysaccharide is $\left(\mathrm{C}_{6} \mathrm{H}_{10} \mathrm{O}_{5}\right)_{\mathrm{n}}$, where $\mathrm{n}$ is the number of linked glucose units indicating the degree of polymerisation and length of the chain. The linkage of the glucose monomers is shown below.

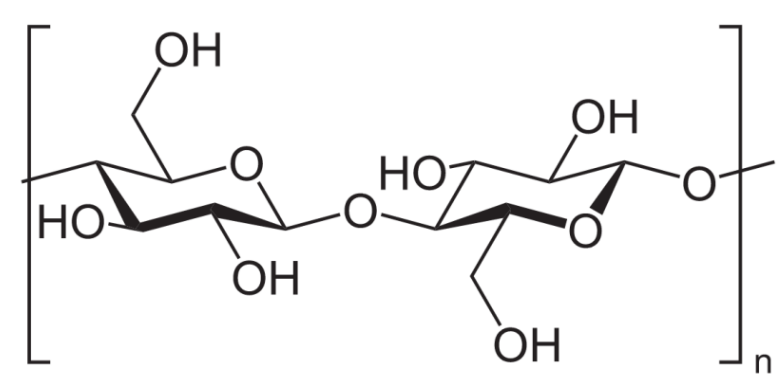

Figure 11 General structure of cellulose

The degree of polymerisation plays a role in the strength and properties of the overall fibre, and so to that of the produced paper. Decreasing chain length below a certain point causes severe reduction in the strength exhibited by the fibre. The amount of polymerisation varies between different plant fibres. In general it is found to be between 600 and 1500 repeat units in commercial wood pulps such as those used in paper production. ${ }^{68}$ This provides reasonably long chain cellulose fibres for paper making. 
As seen in the above representation of the base cellulose unit the linking of the glucose units occurs in fairly linear fashion, almost end on end. This linear structure allows cellulose fibres to align next to each other and associate through the hydroxyl groups with hydrogen bonding. ${ }^{68}$ With long cellulose chains large amounts of hydrogen bonding can occur, creating very strong interactions between the chains. The chains are then held very tightly stacking to create microfibrils of high tensile strength, which gives rigidity to the plants they are derived from. The hydrogen bonding interaction between the cellulose is so strong that it creates crystalline regions within the microfibrils. These regions are also accompanied by regions of poor hydrogen bonding, creating amorphous regions. These amorphous regions are less tightly associated and are thus more permeable than the crystalline regions with the microfibrils. The microfibrils of cellulose associate together also to form macrofibrils to form the cellulose fibres. ${ }^{68,69}$

As already stated, other non-cellulose components play a role in the properties of kraft fibres. Hemicellulose is another carbohydrate derived component of the plant and wood materials used in production. Hemicellullose is a carbohydrate polymer made up of five different sugar components; glucose, mannose, galactose, xylose and arabinose. ${ }^{68}$ Hemicellulose, although also a carbohydrate polymer, exhibits very different properties to cellulose. This huge difference in properties is primarily due to the branched nature of hemicelluloses, caused by the linkages between the varying sugar components and the acidic substituent groups. This prevents the strong hydrogen bonded uniaxial association seen in cellulose, and so the tensile strength of hemicellulose is weak. The short nature of polymer chains in hemicelluloses also decreases its tensile strength. Within plant cells hemicelluloses is relatively abundant. It is found in the cross linking of microfibrils and used in reinforcing the structure. However it is only present in small amounts in paper. Hemicellulose is easily degraded through the pulping process and so does not remain in large quantities in wood pulp used in kraft paper production.

The other major non-cellulose component is lignin. It is of great importance as lignin is the cause of the brown colour characteristic of unbleached kraft fibres. Remaining lignin present 
in wood pulps can undergo removal or oxidation through bleaching to remove the brown colour and create white pulp. Within a woody plant cell the lignin functions as a binder, binding cellulose fibres together by filling the gap between cellulose and hemicellulose increasing strength. ${ }^{68}$ Lignin is amorphous, highly polymerised and branched in structure. ${ }^{68,69}$ This highly polymerised structure of lignin is very large and complex, as well as variable between plant species. This creates issues with characterisation, and as such lignin cannot be fully characterised. Lignin does however primarily consist of three dimensionally linked phenyl propanoid units, with many hydroxyl, carboxylate and methoxy substituents. ${ }^{68}$ An example of a possible lignin structure is illustrated below in Figure 12. The pulping process frees the cellulose fibres from the matrix. ${ }^{68}$ During pulping these three dimensional linkages are mostly destroyed through chemical treatment, however there is a lignin component that remains after pulping.

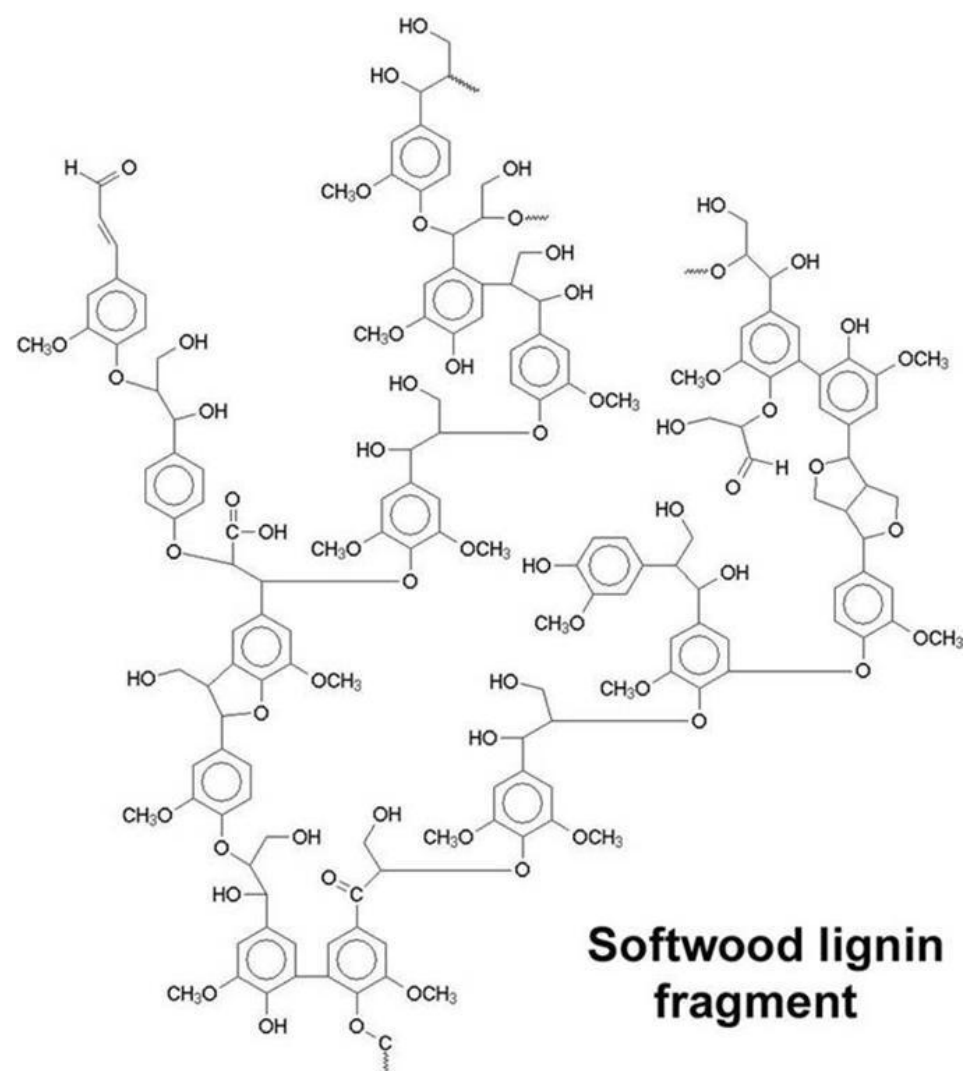

Figure 12 Example of a softwood lignin fragment

The kraft pulping process is a chemical pulping technique. The kraft process is alkaline and involves cooking wood chips in aqueous $\mathrm{NaOH}$ and $\mathrm{Na}_{2} \mathrm{~S}$ solution known as white liquor. ${ }^{68}$ 
White liquor degrades the lignin in the wood chips, freeing the cellulose fibres. The yields for pulp are fairly low typically $40-55 \%$ and so this process dissolves not just the lignin component of the wood chips. Some of the hemicelluloses and cellulose is dissolved also. Approximately $80 \%$ of the lignin, $50 \%$ of the hemicellulose and $10 \%$ of the cellulose is dissolved. $^{68}$

Previously kraft fibres have been functionalised with gold and silver nanoparticles for antimicrobial agents by Thomas Nilsson and Prof J. H. Johnston. ${ }^{70}$ Here it was found unbleached kraft fibres with the phenolic and methoxy groups in conjunction with nitrogen centres in the associated lignin allowed for in situ formation and binding of gold and silver particles. ${ }^{70}$ Cellulose paper fibres are porous, and so enabled the gold and silver nanoparticles to be formed and chemically bound to the fibres themselves. The porous matrix was important in influencing the particle size of the gold and silver. However bleached kraft fibres do not contain phenolic and methoxy groups to initiate chemical bonding of gold or silver to the fibres, as this functionality is removed with the lignin. It was found gold and silver did not readily bond to the oxygen of the cellulose hydroxyl groups either. So it was seen that there was no such composite fibres formed with bleached fibres. ${ }^{70}$

The porous cellulose fibres have been used as a matrix to form antimicrobial agents with these silver and gold nanoparticles already; however bleached fibres could not be used. ${ }^{70}$ Kraft paper fibres will be explored in this project in order to produce a silver/silver halide nanocomposite using the porous nature of the fibres in a similar manner to wool, using both bleached and unbleached kraft fibres, to show that functionality of the fibre is not required to form silver/silver halide fibre nanocomposites.

\subsection{Nanostructured Calcium Silicate}

Nanostructured Calcium Silicate (NCS) is a proprietary material developed by Prof J. H. Johnston through Victoria University of Wellington. ${ }^{71}$ It is a versatile material produced by the reaction of a silica-containing solution with calcium ions at an alkaline $\mathrm{pH}^{71}$ The $3 \mathrm{D}$ 
open microstructure of NCS is similar to that of gypsum desert rose. This structure is formed through Calcium Silicate platelets $5-10 \mathrm{~nm}$ thick and $300 \mathrm{~nm}$ across self-assembling into particles $1-5 \mu \mathrm{m}$ creating an open framework. ${ }^{72,73}$

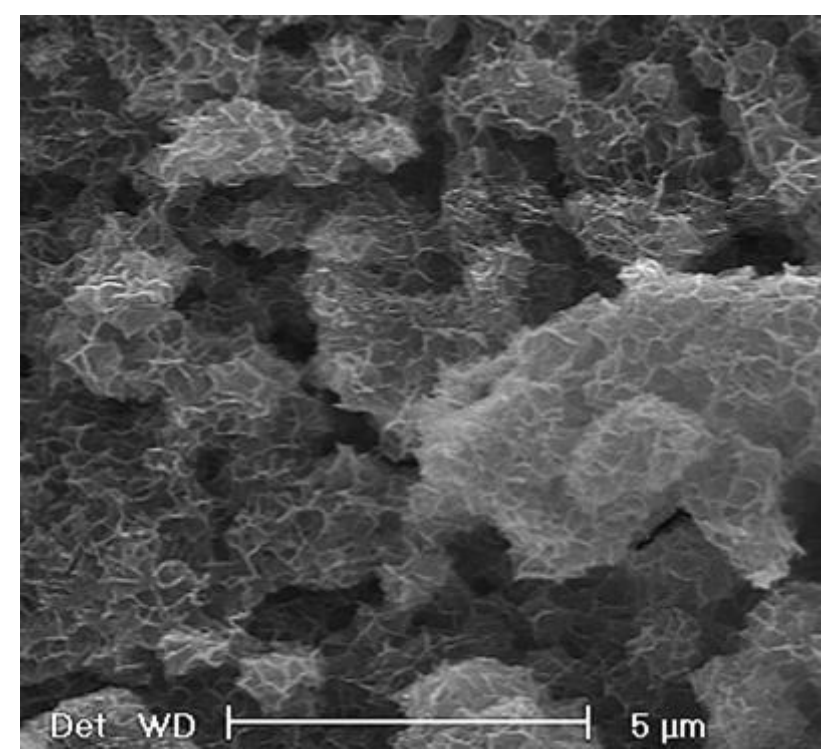

Figure 13 SEM image of the gypsum desert rose structure of $\mathrm{NCS}^{73}$

This open microstructure gives NCS an expansive surface area of 400-600 $\mathrm{m}^{2} \mathrm{~g}^{-1}$ and high oil sorption due to its large pore size, typically $500-600 \mathrm{~g}$ oil $100 \mathrm{~g}^{-1}$ silicate. $^{74}$ The material has a high brightness (ISO brightness of 94-96\%) and excellent whiteness (CIE L* value of 97-98). All these properties exhibited by the NCS have made it successful as filler for newsprint for the paper industry. ${ }^{75}$

The NCS is largely amorphous and without long range order. X-ray diffraction shows peak patterns common to many calcium silicate hydrates giving an indication of the 2 dimensional order in the platelets of the material. At high temperature, greater than $600{ }^{\circ} \mathrm{C}$, the NCS converts to wollastonite, $\mathrm{CaSiO}_{3}$. This suggests a similar structure to wollastonite is present in the NCS, something that is also suggested through solid state Si NMR where the Si environment of NCS is very similar to wollastonite. ${ }^{74}$ It is believed that the structure of NCS consists of backbone chains of $\mathrm{SiO}_{4}{ }^{4-}$ tetrahedra bridged by $\mathrm{Ca}^{2+}$ ions similar to that seen below. (Figure 14) XRF, NMR and XPS have indicated surface bound hydroxyls in the form of silanol groups $(\mathrm{Si}-\mathrm{OH})$ which along with surface $\mathrm{Ca}^{2+}$ ions provide bonding sites for adsorption and functionalisation. Cations such $\mathrm{as}^{+}, \mathrm{Cu}^{2+}$ and $\mathrm{Zn}^{2+}$ have been used to show 
the heavy metal absorption properties of the $\mathrm{NCS}^{72,}{ }^{73}$ The material has also been functionalised with nanoparticles of silver for antimicrobial composites. ${ }^{74}$

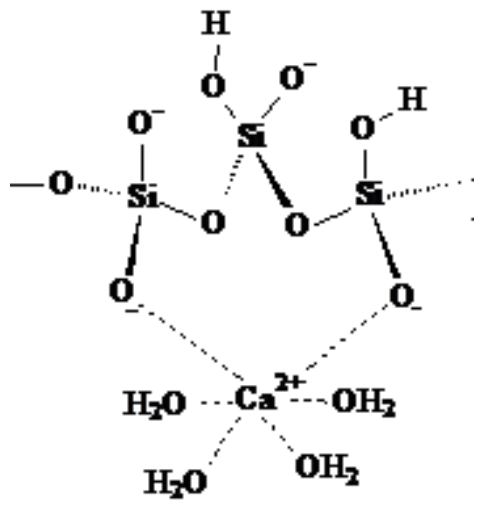

Figure 14 Backbone structure of $\mathrm{NCS}^{73}$

The NCS is synthesised through precipitation from aqueous $\mathrm{Ca}^{2+}$ ions and silica precursors. The silica precursor can be obtained from sodium silicate solution or silica rich geothermal water. These aqueous reactants are mixed at high $\mathrm{pH}$, typically $\mathrm{pH} 11^{72}$ The NCS is created almost immediately, but the more complex microstructure that imparts the high surface area takes time to develop through an ageing process. Once the open microstructure has developed there are problems maintaining the structure throughout the drying process.

The large surface area of the NCS gives it a large liquid-air interface; this in turn leads to a large surface tension. As the framework of the NCS is not a rigid structure but a series of platelets, the large surface tension from the water removal during drying causes a collapse in the structure. ${ }^{72,73}$ This collapse decreases pore size and thus the surface area of the NCS substantially. Drying also changes $\mathrm{Ca}^{2+}$ coordination within the structure substituting the $\mathrm{H}_{2} \mathrm{O}$ for $\mathrm{SiO}$ or $\mathrm{SiOH}$, which can further damage the open microstructure. ${ }^{72}$ One effective method to prevent this collapse of structure when drying is to lower the surface tension of the solvent. Water creates large surface tension through hydrogen bonding throughout the solvent and also with the NCS matrix. If the water is displaced with a solvent of lower surface tension the microstructure of the NCS can be preserved. Ethanol has a surface tension of $22.39 \mathrm{mN} \cdot \mathrm{m}^{-1}$ which is far lower than that of water $72.86 \mathrm{mN} \cdot \mathrm{m}^{-1} \cdot{ }^{72}$ Ethanol is used to displace the water 
during filtering of the NCS before drying takes place, maintaining the open pore structure of the NCS.

As mentioned previously silver has been easily adsorbed into the NCS structure. The silver is bound to silanol groups within the NCS matrix to give $\mathrm{AgOH}$ or $\operatorname{Ag}_{2} \mathrm{O}$. The silver is tethered to the surface of the NCS matrix, preventing excessive leaching and allowing a stable and effective antimicrobial agent to be formed. ${ }^{74}$ Similarly NCS has been functionalised with silver nanoparticles to create effective antimicrobial agents. This was carried out through simple adsorption of the silver nanoparticles to the NCS. NCS Silver nanoparticles were prepared through reduction of $\mathrm{Ag}^{+}$with $\mathrm{NaBH}_{4}$ and stabilised with PVP. These silver nanoparticles were added to pre-prepared NCS slurries and homogenised well in facilitate order to uptake the silver nanoparticles into the NCS structure. ${ }^{76}$ These silver nanoparticle functionalised samples proved to be effective antimicrobial agents. Some colour development occurred in these silver functionalised samples over extended periods of time. This may have been due to $\mathrm{AgCl}$ formation within the $\mathrm{NCS} / \mathrm{Ag}$ samples as the $\mathrm{pH}$ for synthesis is adjusted with $\mathrm{HCl}$. This would tend to lead toward $\mathrm{AgCl}$ functionalised NCS to be a viable composite.

An objective of this part of the research project was to investigate this possibility of forming $\mathrm{Ag} / \mathrm{AgCl}$ nanoparticles within the highly porous NCS structure with the view to create NCS matrix stabilised silver halide plasmonic photocatalysts. Along with NCS, wool and kraft fibres will be used as a matrix to create silver/silver halide nanocomposites. These nanocomposites were compared to synthesised and characterised silver/silver halide nanoparticles. This was to confirm successful incorporation of the silver/silver halide nanoparticles into the various nanocomposite matrices. The following experimental, results and characterisation sections will show that these silver/silver halide nanoparticles and nanocomposites were successfully synthesised. The photocatalytic properties of these silver/silver halide nanoparticles and nanocomposites were then evaluated through the photodegradation of selected organic dye solutions known to be sensitive to photooxidation such as methylene blue. Which is a widely used dye used in assessment of photocatalytic degradation. ${ }^{32,40,45}$ 


\section{Experimental}

\subsection{Experimental Methods}

\subsubsection{Materials}

All chemicals to be used in synthesis were used as received and are analytical grade unless otherwise stated. The silver nitrate $\left(\mathrm{AgNO}_{3}\right)$ was sourced from Scientific and Chemical Supplies, and the polyvinylpyrrolidone (PVP) from Sigma Aldrich. Sodium chloride and sodium bromide were provided by Unilab, and sodium iodide by Pure Science. Calcium hydroxide was from LobaChemie and sodium silicate from Orica Chemicals (N grade). Distilled water was used in all cases. The crossbred wool was sourced from AgResearch Ltd, Christchurch, New Zealand. Bleached and unbleached kraft fibres used in this research project were of the New Zealand Pinus radiata genus, sourced from Tasman Pulp and Paper Company Ltd, Kawerau, New Zealand.

\subsubsection{Preparation of silver/silver halide colloids}

Silver/silver halide nanoparticles solutions were synthesised by the following typical methodology. $0.1 \mathrm{M} \mathrm{NaCl}, \mathrm{NaBr}$ or $\mathrm{NaI}$ solutions were adjusted to acidic conditions. The $\mathrm{pH}$ was adjusted to $\mathrm{pH}=3$ using acetic acid so as to not introduce additional $\mathrm{Cl}^{-}$ions. PVP was added to the solution $(1 \mathrm{ml}, 2 \mathrm{wt} \%)$ as a capping agent to make the solution up to $10 \mathrm{ml}$. This solution was then thoroughly mixed using an IKA high shear homogeniser. $\mathrm{AgNO}_{3}$ solution was added under these high shear mixing conditions, forming the silver halide nanoparticles. The concentration of $\mathrm{AgNO}_{3}$ solution was varied, to give an amount of $100-300 \mathrm{mg} \mathrm{Ag}$ for the colloidal solutions. The formation of silver halides occurs when the $\mathrm{AgNO}_{3}$ is added under mixing. Immediately a cloudy white colour is formed for $\mathrm{AgCl}$ and $\mathrm{AgBr}$ colloidal suspensions. The solutions were then irradiated in a $150 \mathrm{~W}$ unfiltered Xe Arc lamp for 16 hours. After this period of time the $\mathrm{AgCl}$ and $\mathrm{AgBr}$ nanoparticle solutions developed a deep purple colour. This light exposure produces silver nanodomains making silver/silver halide 
nanoparticles. AgI colloidal solutions are a yellow colour when they are formed. This colour is maintained after light exposure.

\subsubsection{Preparation of silver/silver halide wool nanocomposites}

Silver halide wool nanocomposites were synthesised by the following methodology developed by Kelly. ${ }^{67}$ The wool fibres were doped with halide ions, $\mathrm{Cl}^{-}, \mathrm{Br}^{-}$and $\mathrm{I}^{-}$under acidic conditions. This was done by immersing $0.1 \mathrm{~g}$ of wool fibres in $10 \mathrm{ml}$ of $0.1 \mathrm{M} \mathrm{NaCl}$, $\mathrm{NaBr}$ or $\mathrm{NaI}$ solution. The $\mathrm{pH}$ was adjusted to $\mathrm{pH}=3$ using acetic acid. After soaking, the wool fibres were rinsed with distilled water and air dried. To form the silver halide nanoparticles the doped fibres were then soaked in $\mathrm{AgNO}_{3}$ solution. The concentration of $\mathrm{AgNO}_{3}$ solution ranged, to give final concentrations of $100-300 \mathrm{mg} / \mathrm{kg} \mathrm{Ag}$ for the final composite. The formation of silver halides occurs when the fibres are immersed within the $\mathrm{AgNO}_{3}$. The fibres are then removed from the solution after soaking, and washed with distilled water and left to air dry.

$\mathrm{AgCl}$ and $\mathrm{AgBr}$ wool nanocomposites are white in colour when first synthesised. The samples are then left in natural sunlight, behind window glass for a period of three hours. During this period of time the $\mathrm{AgCl}$ and $\mathrm{AgBr}$ wool nanocomposites develop a purple colour. The purple colour is often observed during the first hour of light exposure. This light exposure produces silver nanodomains, making silver/silver halide nanoparticles within the wool fibre matrix. AgI wool nanocomposites formed yellow-coloured nanocomposites, when soaked in $\mathrm{AgNO}_{3}$. When exposed to the same light conditions mentioned above, these nanocomposites showed no further colour development. 


\subsubsection{Preparation of silver/silver halide kraft fibre nanocomposites}

Silver/silver halide kraft fibre nanocomposites were synthesised by the following methodology modified from the successful wool nanocomposite method. Both bleached and unbleached kraft fibres were used for nanocomposite synthesis. Kraft fibres were doped with halide ions, $\mathrm{Cl}^{-}, \mathrm{Br}^{-}$and $\mathrm{I}^{-}$under acidic conditions. This was done by immersing $0.1 \mathrm{~g}$ of homogenised kraft fibres in $10 \mathrm{ml}$ of $0.1 \mathrm{M} \mathrm{NaCl}, \mathrm{NaBr}$ or $\mathrm{NaI}$ solution. The $\mathrm{pH}$ was adjusted to $\mathrm{pH}=3$ using acetic acid. After soaking, the kraft fibres were rinsed with distilled water and filtered for drying. To form the silver halide nanoparticles the doped fibres were then soaked in $\mathrm{AgNO}_{3}$ solution. The concentrations of $\mathrm{AgNO}_{3}$ solution were adjusted, to give final concentrations of $100-300 \mathrm{mg} / \mathrm{kg} \mathrm{Ag}$ for the final composite. The formation of silver halides occurs when the fibres are immersed within the $\mathrm{AgNO}_{3}$. The fibres are then removed from solution after soaking, and washed with distilled water, before being filtered and dried.

$\mathrm{AgCl}$ and $\mathrm{AgBr}$ bleached kraft nanocomposites are white in colour when first synthesised. The samples are then left in natural sunlight, behind window glass for a period three hours. During this period of time, the $\mathrm{AgCl}$ and $\mathrm{AgBr}$ wool nanocomposites develop a purple colour. This colour change is also seen to develop during the first hour at room temperature. This light exposure produces silver nanoregions, making silver/silver halide nanoparticles within the fibre matrix. AgI bleached kraft nanocomposites formed yellow-coloured nanocomposites, when soaked in $\mathrm{AgNO}_{3}$. When exposed to the same light conditions as above, these nanocomposites showed no further colour development. Unbleached $\mathrm{kraft} \mathrm{AgCl}$ and $\mathrm{AgBr}$ nanocomposites are brown in colour when first synthesised, due to the lignin content of the unbleached fibres. After the period of natural sunlight light exposure behind window glass, a dirty purple/brown colour developed. AgI unbleached kraft nanocomposites formed a dirty yellow/brown colour during synthesis, and when exposed to light no further colour development was observed. 


\subsubsection{Synthesis of silver/silver halide nanostructured calcium silicate nanocomposites}

The silver halide NCS nanocomposites were synthesised by the following method. The first step was the synthesis of the NCS matrix. A $500 \mathrm{ml}$ batch of NCS was made from the

following procedure developed by the Prof. J. H. Johnston and group. ${ }^{71-73} \mathrm{Ca}(\mathrm{OH})_{2},(5.79 \mathrm{~g})$ was suspended in $250 \mathrm{~cm}^{3}$ of $0.174 \mathrm{~mol} \mathrm{dm} \mathrm{dm}^{-3} \mathrm{HCl}$ under vigorous stirring. A second solution of sodium silicate $\left(15.91 \mathrm{~g}\right.$ of $\left.27 \mathrm{wt} \% \mathrm{SiO}_{2}\right)$ was made up to $250 \mathrm{~cm}^{3}$ with distilled water. The sodium silicate solution was rapidly added to the calcium hydroxide to create a slurry. This calcium silicate slurry was stirred for one hour further and then aged over night. The NCS was then recovered through vacuum filtration, where it was washed thoroughly with distilled water. To maintain the integrity of the open framework and pore structure of the NCS, by preventing collapse, the NCS was washed with ethanol before it dried out. The lower surface tension of the ethanol compared to water prevented loss of structure. The filter cake was then dried in an oven at $110^{\circ} \mathrm{C}$ overnight.

This bulk batch of NCS then served as the base material for the silver halide NCS nanocomposites. Typical synthesis of silver halide NCS composites was as follows. $0.1 \mathrm{~g}$ of NCS was washed thoroughly with distilled water in order to remove residual $\mathrm{Cl}^{-}$ions from the $\mathrm{HCl}$ solution during NCS synthesis. After washing and filtration, the washed NCS was suspended in $0.1 \mathrm{M} \mathrm{NaCl}, \mathrm{NaBr}$ or $\mathrm{NaI}$ solution and agitated for a 1 hour period. This was followed by filtration, before immersing the halide doped $\mathrm{NCS}$ in $\mathrm{AgNO}_{3}$ solution. The $\mathrm{AgCl}$ and $\mathrm{AgBr}$ NCS nanocomposites formed white nanocomposites when first immersed in the $\mathrm{AgNO}_{3}$ solution. With natural sunlight exposure behind window glass the nanocomposites developed a purple colour, similar to the kraft and wool nanocomposites. AgI NCS nanocomposites immediately formed a yellow nanocomposite, which did not change with light. The final products were washed with distilled water and filtered with ethanol to maintain the open pore structure of NCS. 


\subsection{Characterisation Techniques}

\subsubsection{UV Visible Spectroscopy}

UV Visible Spectroscopy (UV/Vis) was used to monitor the colour development of the silver halide nanocomposites and solutions. There is a characteristic broad visible region absorption that develops when there is partial reduction of the silver halide to silver for form silver/silver halide nanoparticles from $\sim 400-800 \mathrm{~nm}$. This absorption can be easily monitored by $\mathrm{UV} / \mathrm{Vis}$ spectroscopy. UV/Vis spectroscopy may also be used to monitor the typical silver halide absorption profile as an additional confirmation tool for successful silver halide formation. Absorption and reflectance measurements will be carried out as wool, kraft fibre and NCS nanocomposites are opaque. These reflectance measurements were transformed using Kubelka-Monk transformation to the colour yield (K/S). Absorption and reflectance UV/Vis were carried out on a Varian Cary 100 scan spectrometer. Solution samples were analysed by absorption and solid nanocomposite samples by reflectance.

\subsubsection{X-ray Diffraction}

X-ray diffraction (XRD) was used to monitor the composition of the silver halide nanocomposites and colloids. An XRD pattern results from the interaction of $\mathrm{x}$-rays with crystalline substances through diffraction. These diffractions result in a characteristic pattern that can be used to determine the structure and identity of crystalline substances. In this project it was used to determine the presence of crystalline silver halides and silver within the nanocomposite matrices and the nanoparticles produced in solution. All measurements were carried out on an X'Pert Pro diffractometer. The X'Pert Pro uses a Cu source, operating at 45 $\mathrm{kV}$ and $40 \mathrm{~mA}$. Diffraction patterns observed from sample preparation were compared with XRD patterns from the ICDD database. Solution samples were prepared by drop casting onto glass slides. The wool and kraft samples were prepared by mounting the fibres onto the sample holder with kapton tape, with care taken to mount them as flat as possible. NCS samples were prepared through normal powder diffraction methods. 


\subsubsection{Scanning Electron Microscopy}

Scanning electron microscopy (SEM) imaging was carried out on a JEOL $6500 \mathrm{~F}$ field emission gun scanning electron microscope. It is an important characterisation technique for studying the surface morphology of fibres and the silver halide nanocomposites. The high atomic mass of silver and low atomic mass of the fibre and NCS matrices allows backscatter imaging to be employed. The backscattered electron imaging provides good contrast between the nanocomposite matrix (which appears dark) and nanoparticles (which appear light). In order to determine the sample surface morphology and ensure uniformity, images were taken at various positions throughout samples, including cross-sectional analysis.

\subsubsection{Energy Dispersive X-ray Spectroscopy}

Energy dispersive X-ray spectroscopy (EDS) using the JEOL $6500 \mathrm{~F}$ field emission gun scanning electron microscope, was employed to investigate the elemental composition of the silver halide nanoparticles and nanocomposites. EDS was able to show the concentration of different elements on the surface of a nanocomposite sample. EDS mapping provides information on areas with high concentrations of elemental Ag, showing areas where nanoparticles are located. Overlaying Ag with areas of high concentration of halide can confirm that silver halide particles are formed. Cross-sectional analysis with EDS also shows that the silver halide nanoparticles are located within the matrix as well as on the surface.

\subsubsection{Transmission Electron Microscopy}

Transmission Electron Microscopy (TEM) is a complementary technique to SEM. It does have a higher resolution, allowing for more highly detailed morphological information. This gave insight into the structure and morphology of the silver halide nanoparticles themselves. TEM analysis of the $\mathrm{Ag} / \mathrm{AgCl}$ nanoparticles was carried out on a JEOL 2011 high-resolution instrument with $\mathrm{a} \mathrm{LaB}_{6}$ filament operated at a $200 \mathrm{kV}$ acceleration voltage. Imaging was done at varying positions throughout the sample so that a uniform representation of the samples 
was obtained. This gave size and morphological information. Electron diffraction was also carried out as a conformational tool, to determine whether both crystalline $\mathrm{Ag}$ and $\mathrm{AgCl}$ are present within the nanoparticles. Scanning transmission electron microscopy (STEM) was also used in order to employ EDS for compositional information about the nanoparticles viewed under the TEM.

\subsubsection{Determination of Photocatalyic Activity}

Photocatalytic activity was determined through the breakdown of the organic dye methylene blue (MB) a common dye used in photocatalytic determination as mentioned previously. ${ }^{32,40,}$ ${ }^{45} 5 \mu \mathrm{M}$ MB solution was used to determine the photocatalytic activity of the silver/silver chloride nanoparticles. $0.4 \mathrm{mg}$ of the nanoparticles were suspended in the MB solution and stirred in dark conditions overnight to reach equilibrium. The solution was then irradiated by a $75 \mathrm{~W}$ Xe arc lamp, and the MB monitored at time intervals via UV/Vis spectroscopy to measure degradation time. This was compared with the decomposition rate of MB solution only in light, and $\mathrm{MB}$ in the presence of bulk $\mathrm{AgCl}(0.4 \mathrm{mg})$.

Photocatalytic activity of the silver chloride nanocomposites was monitored by reflectance spectroscopy due to the solid nature of the matrices used in synthesis. The decrease in absorbance intensity of $10 \mu \mathrm{M}$ MB solution was used to determine the photocatalytic activity of the silver/silver chloride nanocomposites. An Ag content of $0.4 \mathrm{mg}$ of the nanocomposite was suspended in $10 \mathrm{ml}$ of $\mathrm{MB}$ solution and stirred in dark conditions overnight to reach equilibrium. The nanocomposite was then filtered and dried, and was then irradiated by a $75 \mathrm{~W}$ Xe arc lamp, and the MB monitored at time intervals via UV/Vis reflectance spectroscopy to measure degradation time. This was compared with the decomposition rate of the MB dyed blank matrix. This gave an indication of photocatalytic activity through the rate increase of MB degradation. 


\section{Results and Discussion}

\subsection{Silver/Silver Halide Colloids}

The first step of this project was to synthesise and characterise silver/silver halide nanoparticles as stable colloids. The colloids were prepared and their colours and UV/Vis absorption and reflectance spectra were compared to the silver halide wool nanocomposites made by Kelly. ${ }^{67}$ Using the synthesis method described previously in the experimental section, colloids of $\mathrm{AgCl}, \mathrm{AgBr}$ and $\mathrm{AgI}$ were made. $\mathrm{AgCl}$ and $\mathrm{AgBr}$ nanoparticle colloids stabilised with PVP were made by a method similar to that used by Choi et al. ${ }^{45}$ This method involved synthesising silver halide nanoparticles stabilised with PVP and then converting them to silver/silver halide nanoparticles through irradiation with a Xe arc lamp. In the synthesis the addition of the $\mathrm{AgNO}_{3}$ to the $\mathrm{NaCl}$ and $\mathrm{NaBr}$ solutions caused a white cloudy solution to form. This white colour is indicative of $\mathrm{AgCl}$ and $\mathrm{AgBr}$ colloids being formed. When the colloids were irradiated in the Xe arc lamp, a purple colour developed. This development of a purple colour was what Kelly had seen in her $\mathrm{AgCl}$ and $\mathrm{AgBr}$ wool nanocomposites. ${ }^{67}$ The development of this purple colour is due to a broad visible region absorption, present after the $\mathrm{AgCl}$ and $\mathrm{AgBr}$ particles are exposed to light.

The UV/Vis absorption spectra obtained for the colloids (Figure 15, 16, 17) are consistent with semi-conductor silver halides. Silver chloride before being exposed to light shows the typical UV/Vis absorbance from $280-300 \mathrm{~nm}$ wavelength for the direct band gap of the $\mathrm{AgCl}$, and a small peak at $380 \mathrm{~nm}$ for the indirect band gap of $\mathrm{AgCl}$. After light exposure, it is seen that a visible absorption developed in the sample from $450-800 \mathrm{~nm}$. (Figure 15) This turns the $\mathrm{AgCl}$ colloid from the cloudy white of $\mathrm{AgCl}$ to purple. This change in colouration is caused by the formation of $\mathrm{Ag}$ nanodomains on the surface of the $\mathrm{AgCl}$ nanoparticle. These Ag nanodomains will be shown to be present in the following characterisation sections. These silver domains are formed through the partial reduction of $\mathrm{Ag}^{+}$to $\mathrm{Ag}^{0}$, through the photographic process. ${ }^{45}$ The mechanism proposed by Gurney and Mott discussed in the introduction, demonstrates the process of photolysis through which these silver nanodomains are formed. ${ }^{6}$ 


$$
\begin{gathered}
\mathrm{Ag}^{+} \mathrm{X}^{-}+\mathrm{h} v \rightarrow \mathrm{Ag}^{+}+\mathrm{X}+\mathrm{e}^{-} \\
\mathrm{Ag}^{+}+\mathrm{e}^{-} \rightarrow \mathrm{Ag}^{0}
\end{gathered}
$$

On the nanoscale photocatalytic coversion of $\mathrm{Ag}^{+}$to $\mathrm{Ag}^{0}$ does not go through to completion. Instead it produces small nanodomains of $\mathrm{Ag}^{0}$ on the surface of the $\mathrm{AgX}$ nanoparticle, rather than completely convert the $\mathrm{AgX}$ nanoparticle to an $\mathrm{Ag}^{0}$ nanoparticle. This is known as a self-sensitisation process, where partial conversion of $\mathrm{Ag}^{+}$occurs. As we saw in the models of this in the introduction, at a certain point this reduction is prevented due to effective electron-hole separation occurring when a photon is absorbed. ${ }^{46,47,77}$ This effective separation prevents the reduction of $\mathrm{Ag}^{+}$and instead promotes radical formation. This is the basis for the photocatalytic activity of the silver/silver halide nanoparticles.

Not only does the formation of silver nanodomains impart photocatalytic properties to the silver halide nanoparticles, it also induces a colour change. It has been discussed that on the nanoscale silver exhibits interesting optical properties. The optical properties of silver nanoparticles differ to bulk due to the phenomenon of SPR. $\mathrm{AgCl}$ nanoparticles do not display SPR due to their semiconducting nature as they have insufficient free conduction band electrons. However, silver nanoparticles do display SPR due to their metallic nature and their nanosize. The silver nanodomains on the silver chloride nanoparticles display SPR in a similar manner to silver nanoparticles. Typically silver nanoparticles show a SPR band at $420 \mathrm{~nm}$ giving them a yellow colour. However, the colour exhibited is dependent on the size, shape and dielectric constant of the nanoparticles. ${ }^{27,28}$ The silver nanodomains are red shifted SPR due to the dielectric constant of the $\mathrm{AgCl}$ surface, this leads to absorption at longer wavelengths than the usual $420 \mathrm{~nm}$ from Ag nanoparticles in an aqueous environment. ${ }^{24}$ This gives rise to the purple colour seen after light irradiation. Secondly, as it will be seen in the TEM analysis of the $\mathrm{Ag} / \mathrm{AgCl}$ nanoparticles, the silver nanodomains take on a variety of shapes and sizes.

Shape and size distribution create a variety of SPR shifts. Typically as the size of the nanoparticles increase the SPRB is red shifted. The mixture of sizes provides varying degrees of shifted SPRB, contributing to the broad visible region absorption from the silver nanodomains. The shapes of the silver nanodomains are a mixture of near spherical and less 
regular shapes, as well as a mixture of agglomerates and discrete regions. This also affects SPR, non spherical particles display more than one SPRB as discussed in the introduction to SPR. This second SPRB is typically red shifted also usually due to an increase in size or aspect ratio of the nanoparticle. ${ }^{20}$ Agglomeration affects the position of SPRBs in a similar way to changes in aspect ratio and increasing particle size. This is due to SPRB coupling, when this occurs neighbouring nanodomains share their SPRBs allowing plasmon oscillation across the length of both domains. Effectively this increases the aspect ratio, red shifting the SPRB. This variety of shapes of the silver nanodomains provides even more variation in SPR to contribute to the broad visible region absorption. The combination of these effects, red shifts and broadens the silver SPRB to give the broad visible absorption seen in the UV/Vis spectra of the silver/silver halide nanoparticles.

$\mathrm{AgBr}$ colloids after light exposure also develop a visible absorption, for the same reasons discussed previously. As to be expected with a visible region absorption like the $\mathrm{Ag} / \mathrm{AgCl}$ samples, the $\mathrm{AgBr}$ nanoparticles develop a purple colour after light exposure. AgI colloidal samples did not form a purple colouration with light exposure, and unlike the $\mathrm{AgBr}$ and $\mathrm{AgCl}$ colloids are not white when first synthesised. The AgI colloids are yellow and this colour remains despite light exposure. The typical AgI absorption spectrum was observed with the two large absorbancies < $450 \mathrm{~nm}$ due to the lower band gap allowing for lower energy light to allow excitation. (Figure 17) However there is a slight increase in absorbance in the $350-$ $450 \mathrm{~nm}$ wavelength range, after being exposed to light. This is due to a slight conversion of the $\mathrm{AgI}$ to $\mathrm{Ag} / \mathrm{AgI}{ }^{49} \mathrm{AgI}$ displays a resistance to photolysis unlike $\mathrm{AgCl}$ and $\mathrm{AgBr}$. The photolytic mechanism for $\mathrm{AgI}$ is the same as $\mathrm{AgCl}$ and $\mathrm{AgBr}$, however the $\mathrm{AgI}$ rate of photolysis is slow. The mobile $\mathrm{Ag}^{+}$ions migrate throughout the $\mathrm{AgI}$ lattice to form $\mathrm{Ag}^{0}$ defects, but the large $\mathrm{I}^{-}$ions formed simultaneously in the process are also largely retained. The $\mathrm{I}^{-}$has a slow migration rate through the lattice and so can easily reform AgI by re-oxidising the $\mathrm{Ag}^{0}$. 8,9 


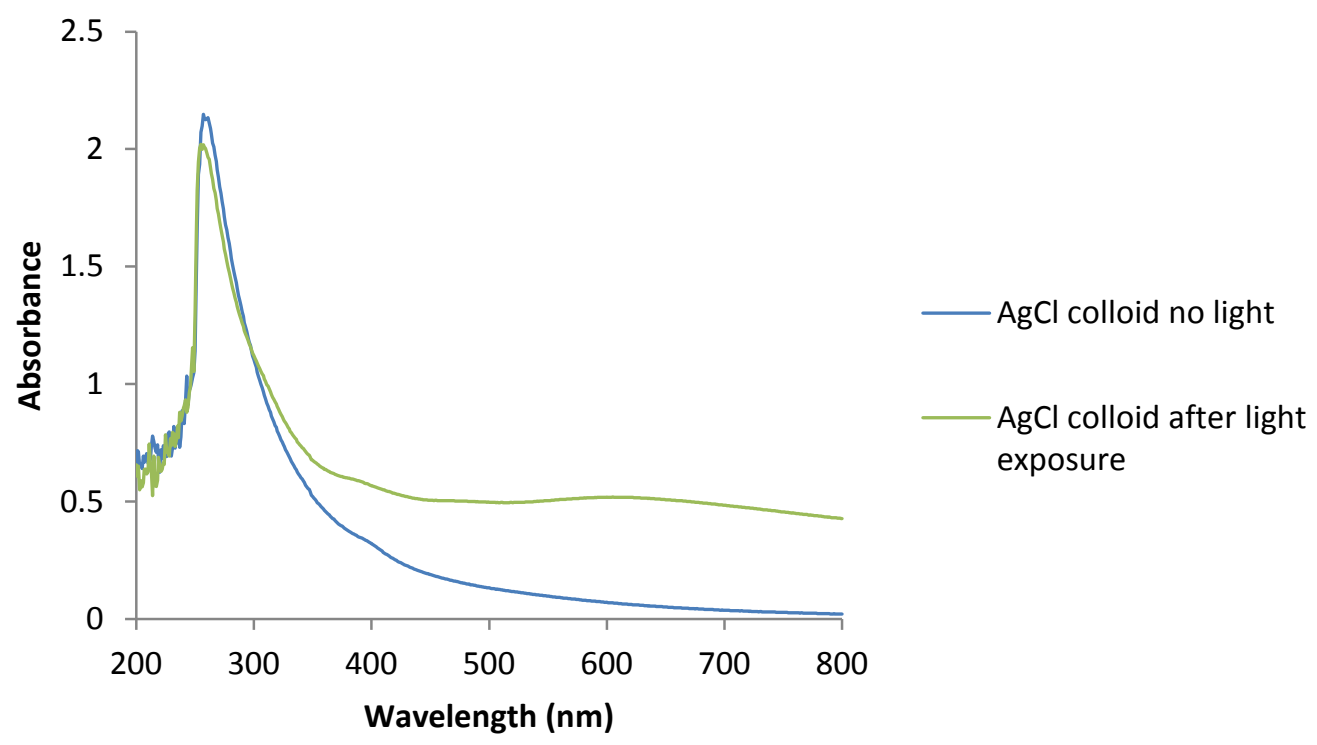

Figure $15 \mathrm{UV} / \mathrm{Vis}$ spectra of $\mathrm{AgCl}$ colloid before and after light exposure

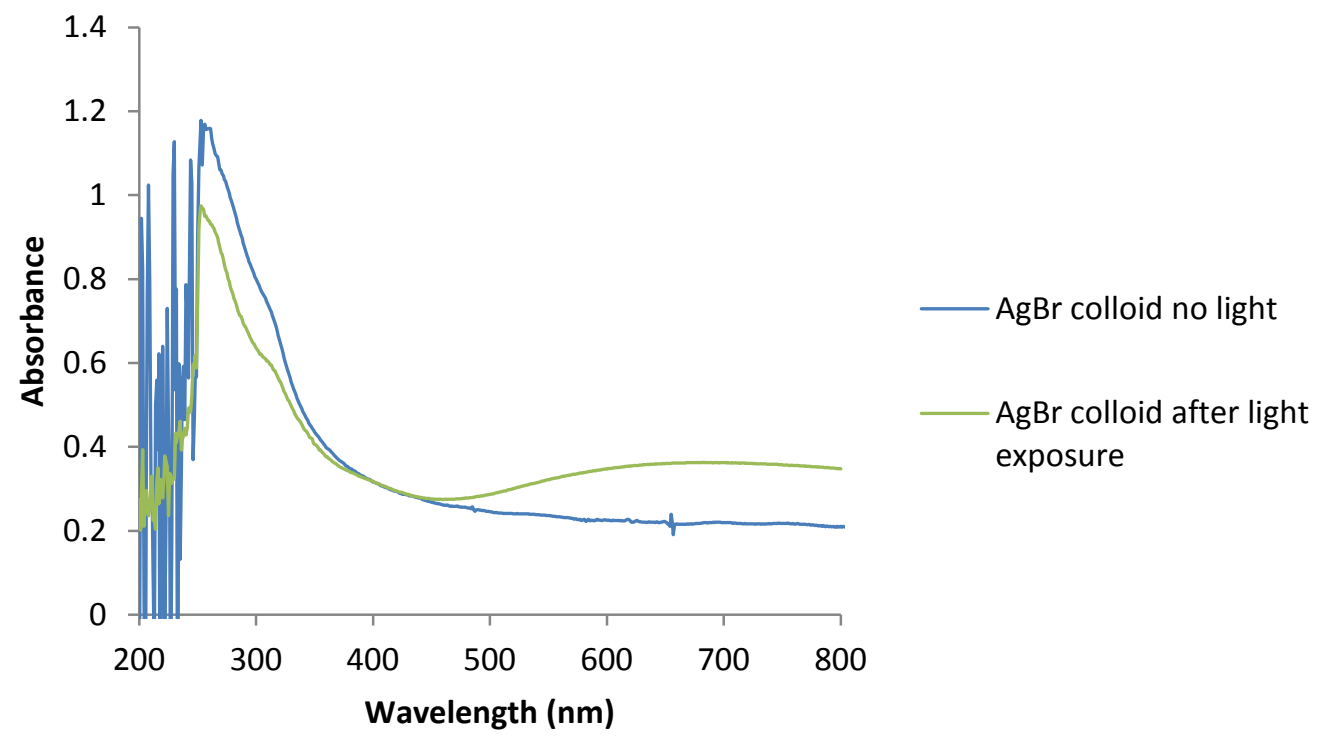

Figure $16 \mathrm{UV} / \mathrm{Vis}$ spectra of $\mathrm{AgBr}$ colloid before and after light exposure 


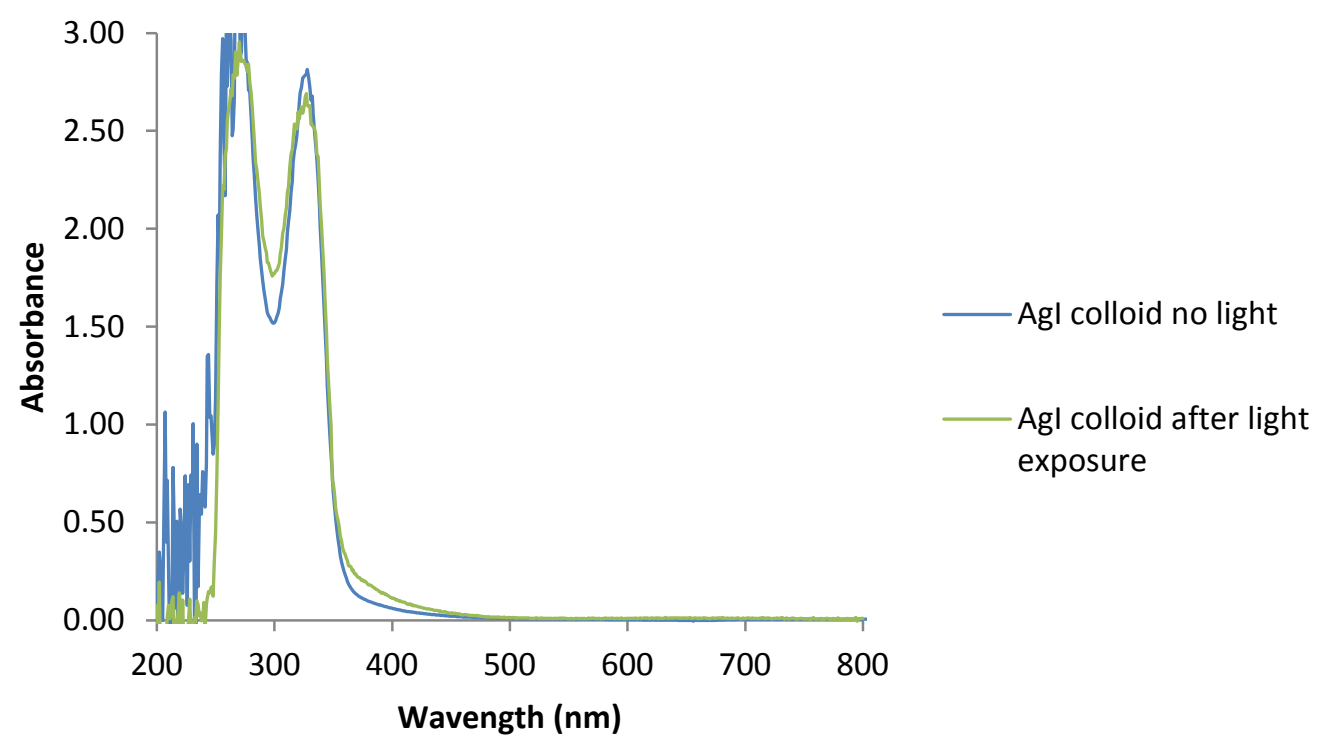

Figure 17 UV/Vis spectra of AgI colloid before and after light exposure

XRD analysis was carried out on the silver halide colloids. This analysis showed that for each $\mathrm{AgCl}, \mathrm{AgBr}$ and $\mathrm{AgI}$ were the only products of the respective synthesis methods. The colour change shown by the $\mathrm{AgCl}$ and $\mathrm{AgBr}$ colloids when exposed to light was also characterised with XRD analysis. An $\mathrm{AgCl}$ colloid sample was synthesised and prepared for XRD analysis in the absence of light. The XRD pattern of this sample can be seen below. (Figure 18) The only phase present in the pattern is that of $\mathrm{AgCl}$. When exposed to light however as seen in figure 19 there is a small additional peak in the diffraction pattern. This small peak correlates to the $\mathrm{Ag} \mathrm{111.} \mathrm{This} \mathrm{shows} \mathrm{that} \mathrm{with} \mathrm{light} \mathrm{exposure} \mathrm{a} \mathrm{small} \mathrm{amount} \mathrm{of} \mathrm{AgCl}$ is converted to $\mathrm{Ag}$, which is responsible for the development of the broad visible absorption seen in the $\mathrm{AgCl}$ and $\mathrm{AgBr}$ colloid samples. 


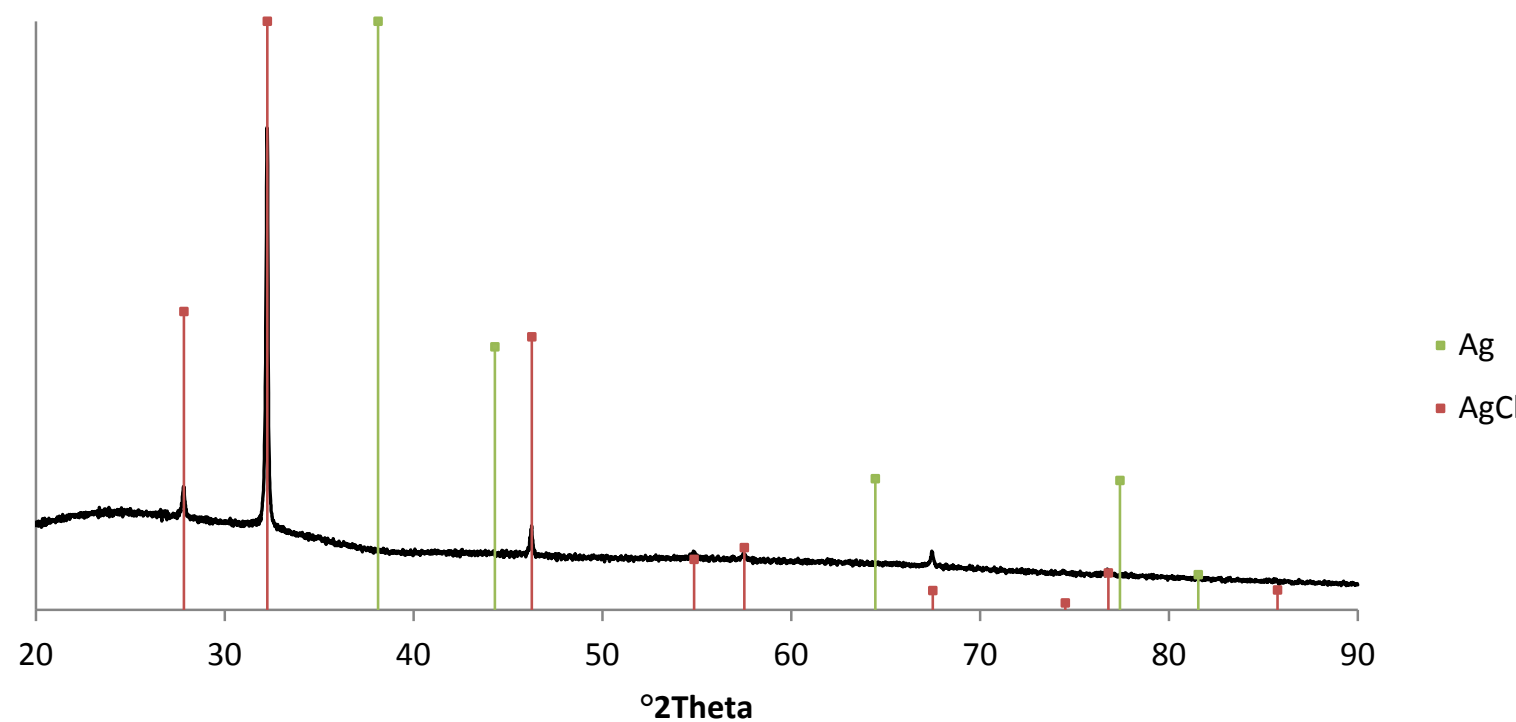

Figure $18 \mathrm{XRD}$ pattern of $\mathrm{AgCl}$ colloid before light exposure

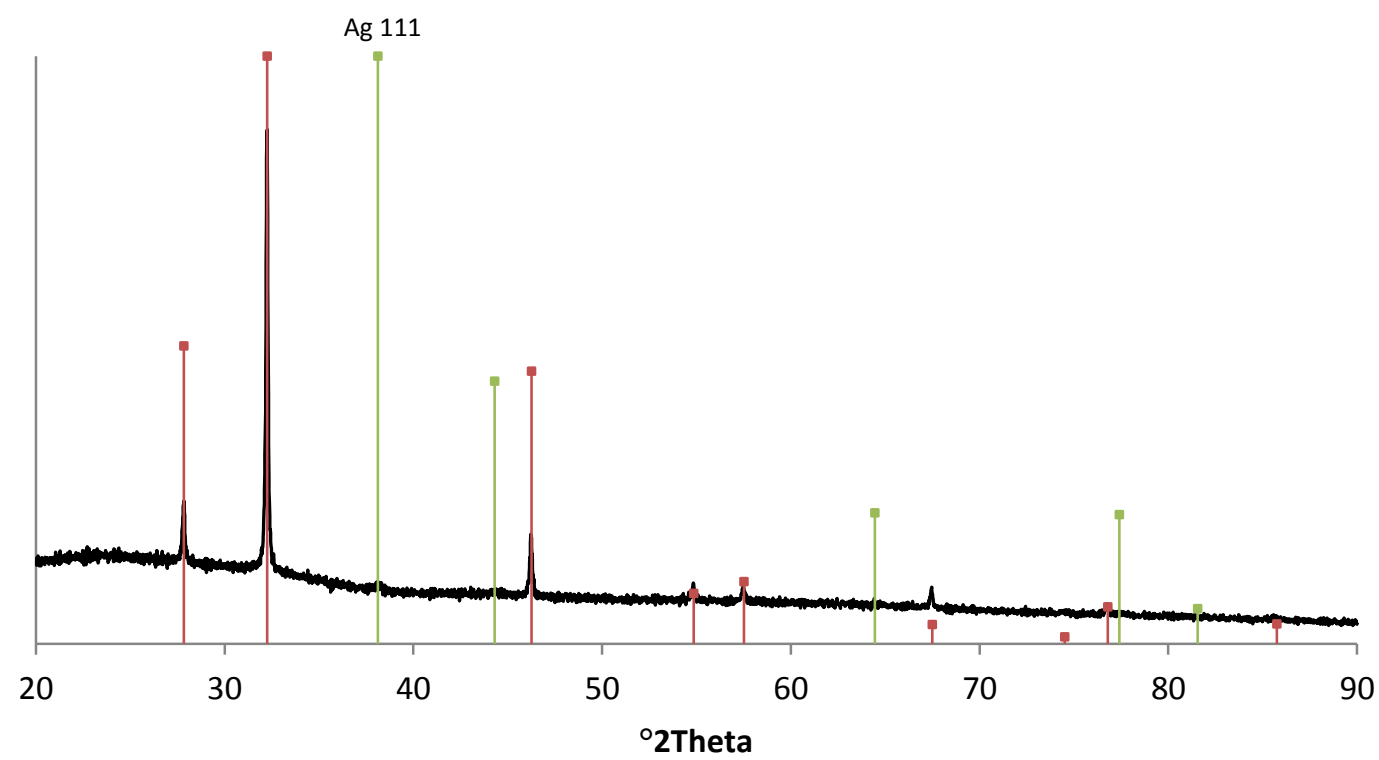

Figure $19 \mathrm{XRD}$ pattern of $\mathrm{AgCl}$ colloid after light exposure

$\mathrm{Ag} / \mathrm{AgBr}$ nanoparticles showed an XRD pattern that confirmed that crystalline $\mathrm{AgBr}$ was present. (Figure 20) Also with the sample exposed to light, there is an Ag 111 peak present showing small amounts of crystalline Ag is also present within the sample. The unassigned peaks in the $\mathrm{Ag} / \mathrm{AgBr}$ diffraction pattern are due to calcium silicate contamination, a common 
problem in the laboratory. AgI has a wurtzite crystal structure, giving a different XRD pattern to the $\mathrm{AgCl}$ and $\mathrm{AgBr}$ diffraction patterns, which are both fcc. The XRD pattern of the AgI nanoparticles after light exposure (Figure 21) confirmed the presence of crystalline AgI. However no Ag 111 peak was detected. The AgI sample would have amounts of Ag lower than that of the $\mathrm{AgCl}$ and $\mathrm{AgBr}$ samples, giving it a lower $\mathrm{Ag} 111$ peak than the already very small $\mathrm{AgCl}$ and $\mathrm{AgBr}$ nanoparticle $\mathrm{Ag} 111$ peak. This would be very difficult to observe.

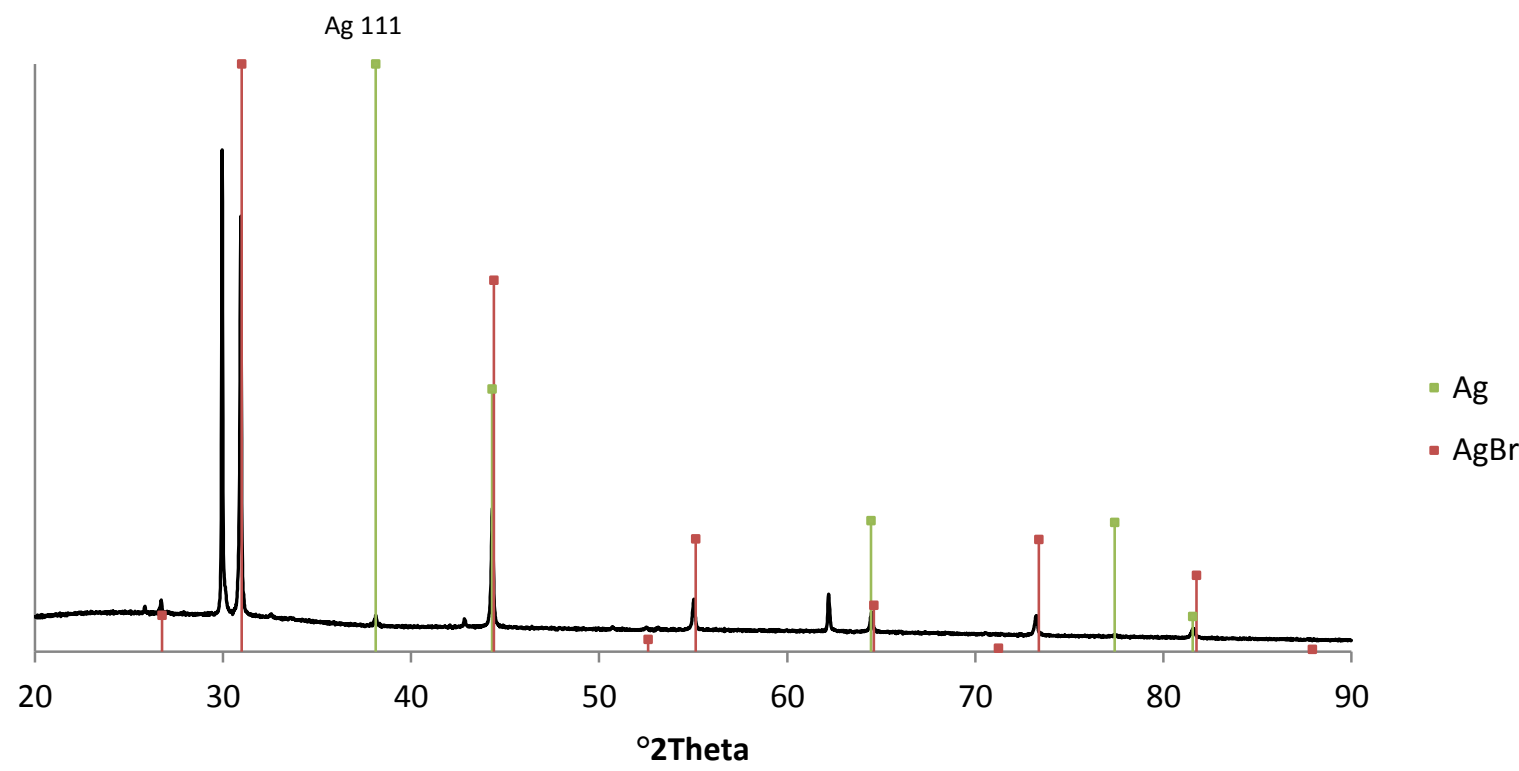

Figure 20 XRD pattern of $\mathrm{AgBr}$ colloid after light exposure

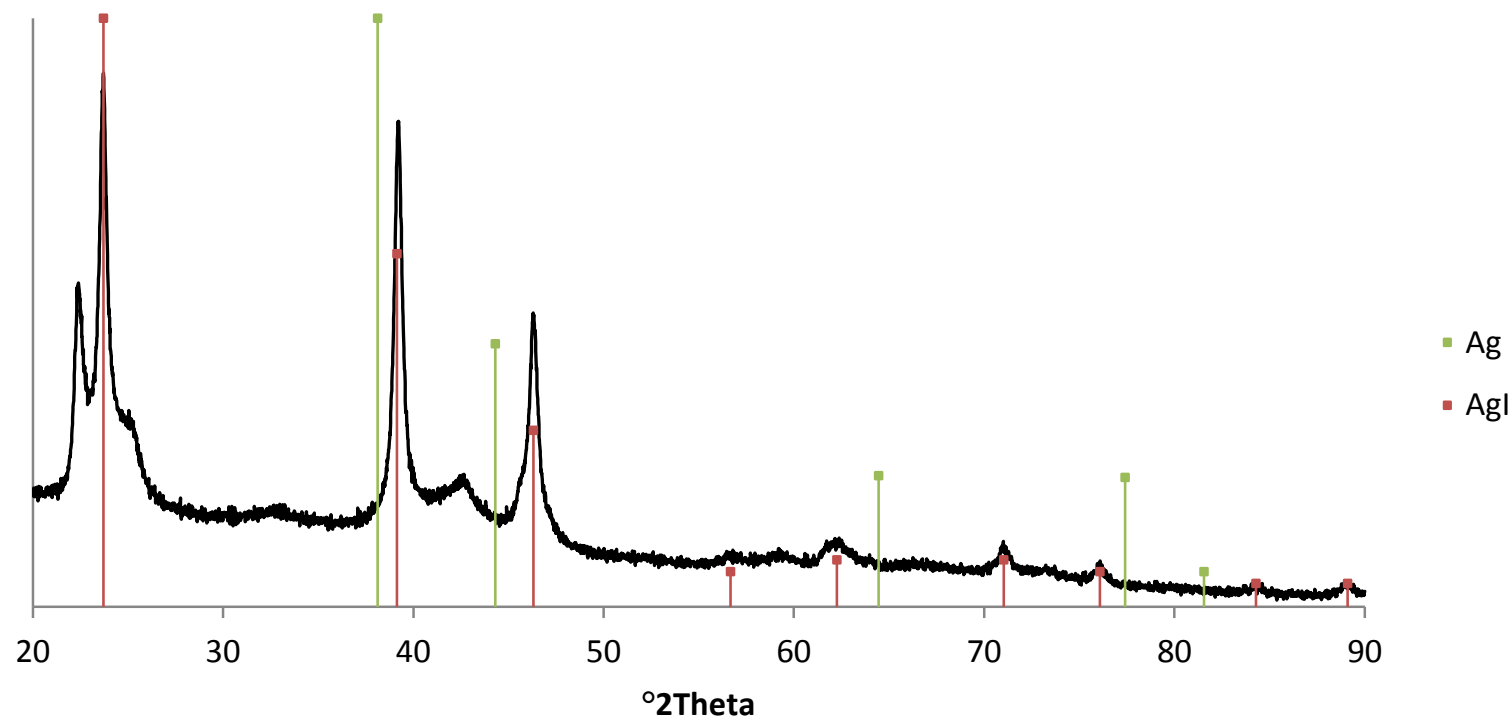


Transmission electron microscopy was used to further characterise the $\mathrm{Ag} / \mathrm{AgCl}$ nanoparticles that had been exposed to light during synthesis. TEM is a very useful tool for studying the size and shape of the $\mathrm{Ag} / \mathrm{AgCl}$ nanoparticles. The TEM clearly shows that cubic particles with darker regions on the surface and edges of the central cubic structure. The particles themselves were difficult to image due to the electron beam causing increased decomposition of the $\mathrm{AgCl}$ nanoparticles. The dark regions would move and grow under the electron beam. However some good images of the $\mathrm{Ag} / \mathrm{AgCl}$ nanoparticles were obtained. In figure $22 \& 23$ it can be seen that the particles have the generally expected cubic structure of $\mathrm{AgCl}$ with the addition of some interesting near spherical surface particles and agglomerates on the surface and edges of the particles. These dark regions have been previously identified as Ag nanodomains in the literature. ${ }^{45,77}$ Typical particles are seen below in figure 22 . There was a range of particle sizes from approximately $50-100 \mathrm{~nm}$ observed. The silver nanodomains were a range of sizes and shapes, from very small discrete domains, to large agglomerates ranging between $5 \mathrm{~nm}$ and over $20 \mathrm{~nm}$.

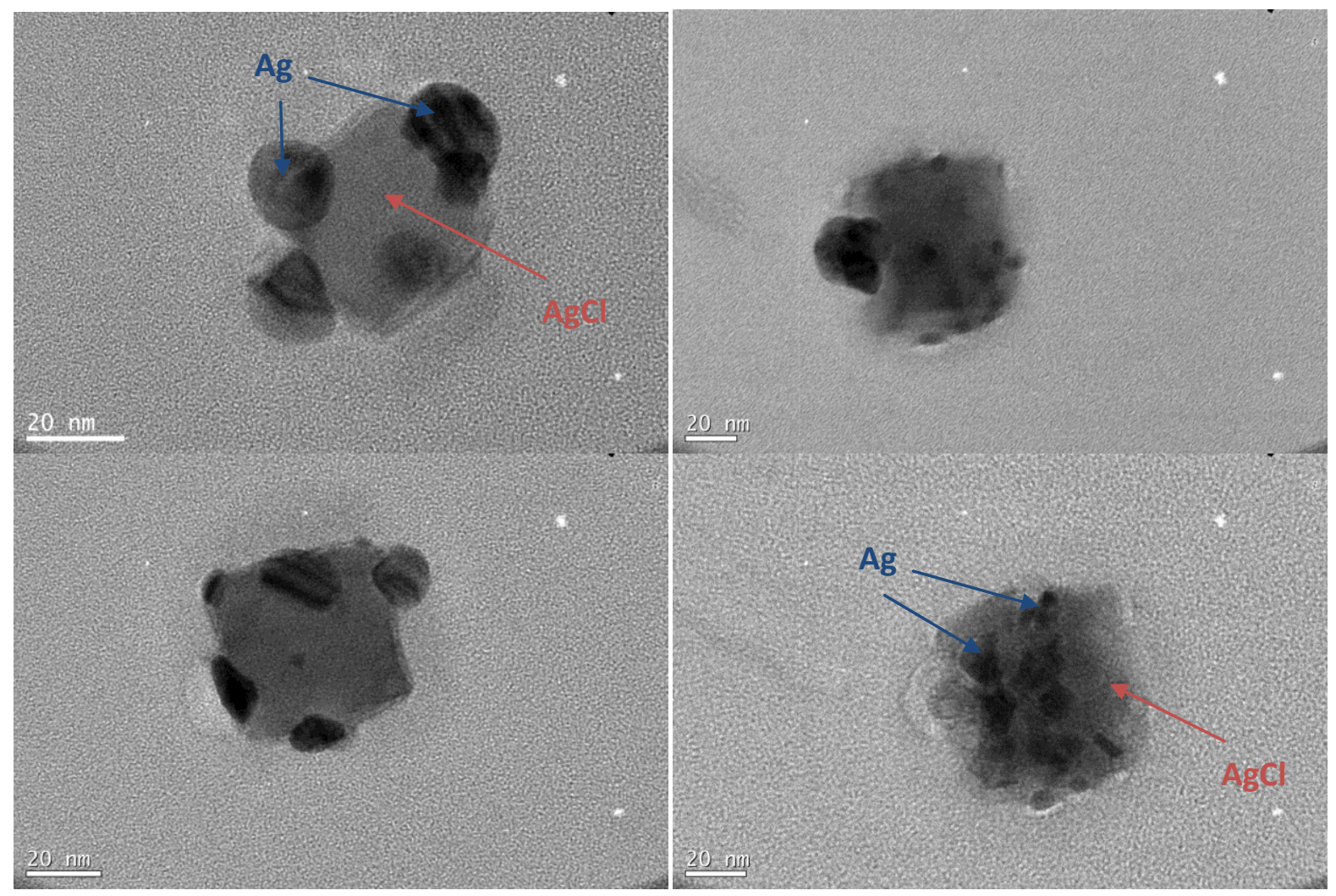


Under the TEM it was possible to view the movement of the of the darker $\mathrm{Ag}^{0}$ nanodomains of the $\mathrm{Ag} / \mathrm{AgCl}$ nanoparticle on the surface of the central cubic $\mathrm{AgCl}$ region. The images below in figure 23 show the agglomeration of these dark $\mathrm{Ag}$ nanodomains of the $\mathrm{Ag} / \mathrm{AgCl}$ nanoparticle under the electron beam. This series of images was taken over the period of two minutes. It can be seen that the smaller regions are slowly incorporated into the larger region, similar to an Ostwald ripening mechanism. This is due to the electron beam causing further photolysis of the $\mathrm{AgCl}$ to $\mathrm{Ag}^{0}$ nanodomains. The lattice fringes that are observed also appear to be changing throughout the process. The cubic central $\mathrm{AgCl}$ crystal that this is occurring on remains stable throughout the process. This difference in behaviour shows that there are two different phases within the one nanoparticle. Having two different phases is in agreement with forming silver nanodomains on the $\mathrm{AgCl}$ nanoparticle during light exposure to create $\mathrm{Ag} / \mathrm{AgCl}$ nanoparticles. This also shows that self-sensitisation is occurring, as the particle does not fully convert to a single phase under the electron beam.
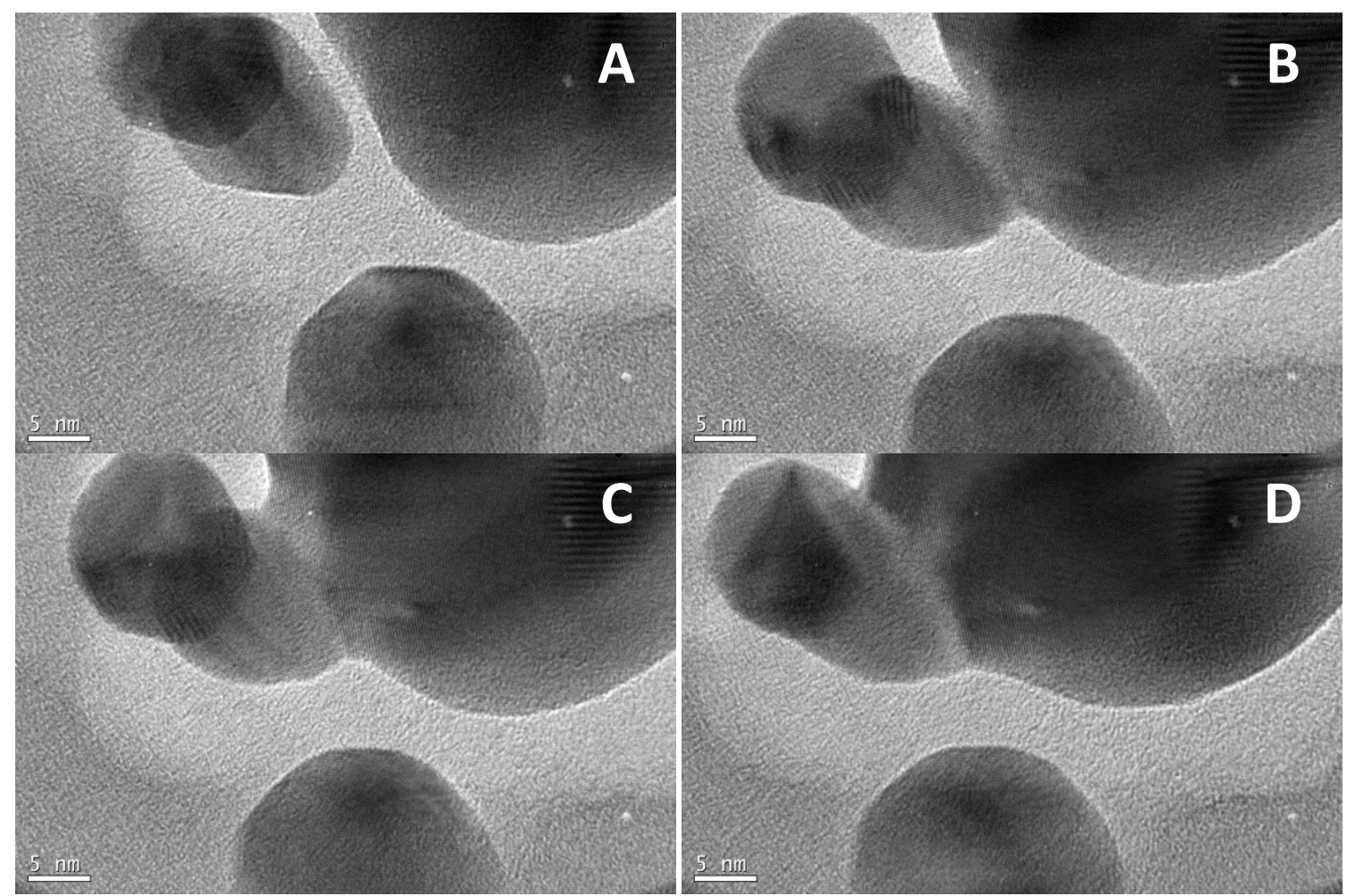

Figure 23 TEM of an $\mathrm{Ag} / \mathrm{AgCl}$ nanoparticle over time A) $0 \mathrm{~s} \mathrm{B)} 30 \mathrm{~s}$ C) 60 s D) $120 \mathrm{~s}$ 
Electron diffraction was carried out on TEM samples of the $\mathrm{Ag} / \mathrm{AgCl}$ colloid. (Figure 24) The diffraction pattern that was obtained shows that there are two phases present within the nanoparticles. The particles do not consist solely of $\mathrm{AgCl}$. Although $\mathrm{AgCl}$ is the dominant pattern present from the diffraction there is also diffraction seen from the $\mathrm{Ag} 111$ and $\mathrm{Ag} 220$ planes. This is further evidence of $\mathrm{Ag} / \mathrm{AgCl}$ nanoparticle synthesis. It was not possible to conclusively determine whether the darker regions of the particle are Ag and the cubic centre $\mathrm{AgCl}$ from this specific diffraction pattern. However, as mentioned it has been determined in the literature previously that this is the case. ${ }^{45,77}$ Of note is the lack of $\mathrm{Ag} 200$ diffraction peak. This is due to the preferential orientation, where the Ag 200 has been suppressed and the $\mathrm{Ag} 220$ has been enhanced from being viewed down the 111 plane. This is due to the $\mathrm{Ag} / \mathrm{AgCl}$ nanoparticle being viewed in one preferential orientation in the electron diffraction. The unassigned diffraction rings have been found to be calcium silicate contamination within the sample. This is also confirmed by STEM and EDS where the samples showed Si present, showing that this is the contamination. (Figure 25)

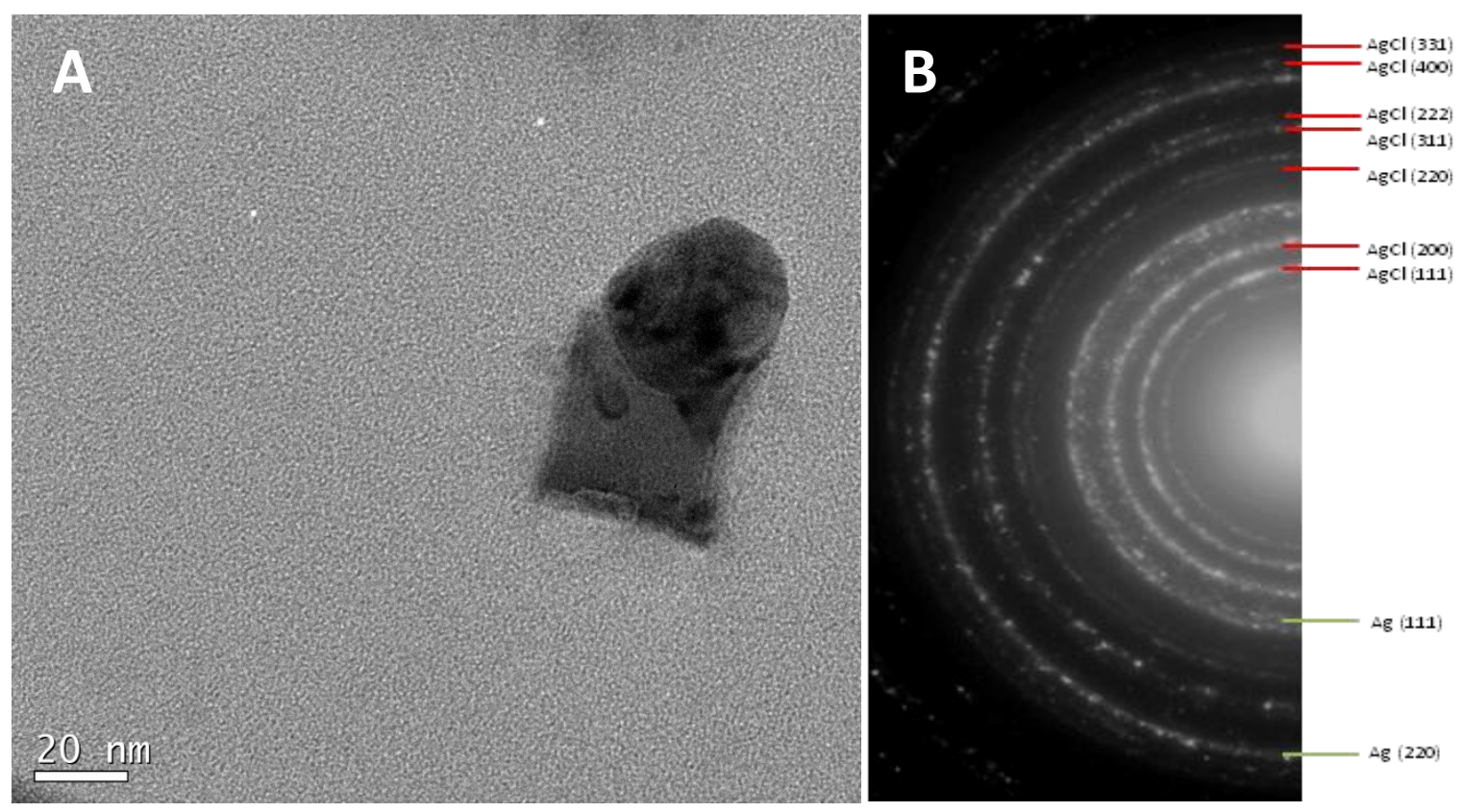

Figure $24 \mathrm{AgCl}$ exposed to light nanoparticle A) TEM image of diffraction sample B) Electron diffraction of nanoparticle 


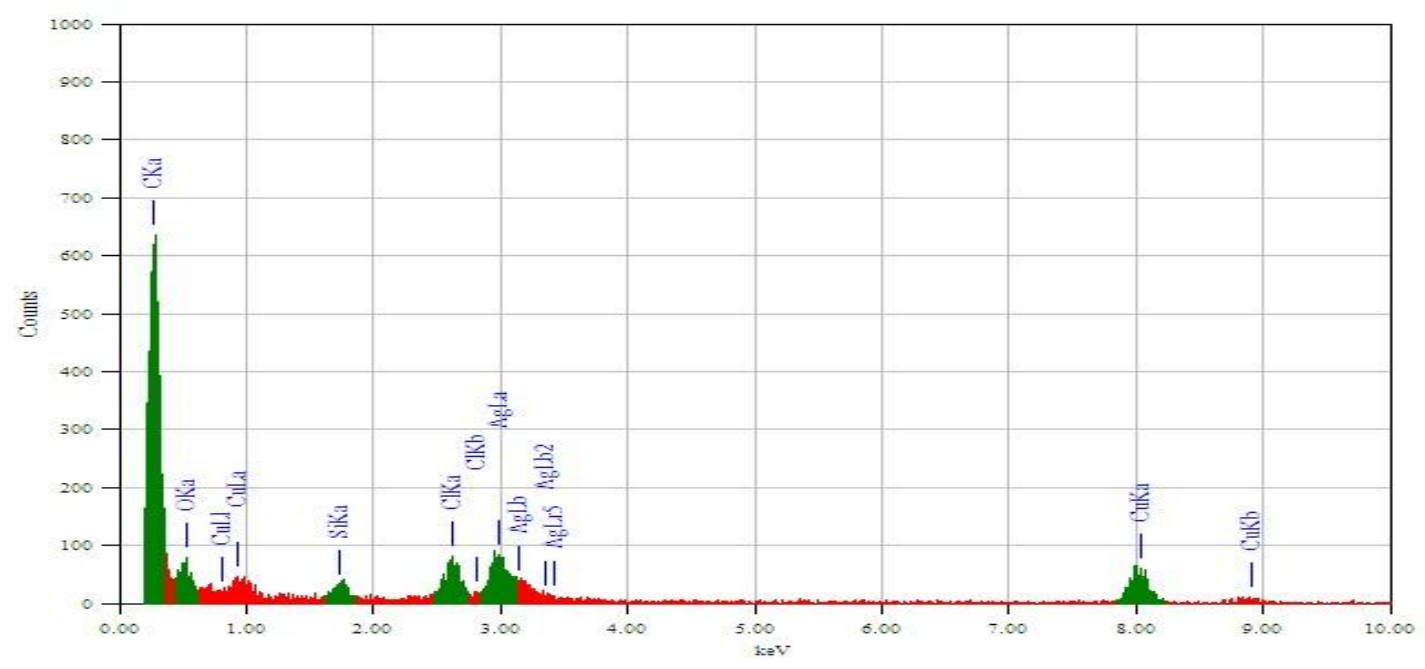

Figure 25 STEM EDS of TEM AgCl nanoparticle sample

In summary the UV/Vis absorption spectra of the silver/silver halide nanoparticles and the colour observed for the samples indicate that the nanoparticles are similar in nature to the silver halide wool nanocomposites made by Kelly. ${ }^{67}$ XRD showed the formation of the silver halides, as well as for the crystalline Ag being present after light exposure. The TEM also showed interesting particle morphology of an $\mathrm{AgCl}$ cube with surface $\mathrm{Ag}$ nanodomains. The electron diffraction confirmed that both silver chloride and silver are present within the nanoparticle, and not separate particles of each. The small nanodomains of $\mathrm{Ag}^{0}$ on the $\mathrm{AgCl}$ nanoparticle exhibit SPR effects giving rise to the colour seen in the nanoparticles. The characterisation has shown that $\mathrm{Ag} / \mathrm{AgCl}$ particles are formed through the synthesis method. These $\mathrm{Ag} / \mathrm{AgCl}$ particles are known photocatalysts. They can be used as a comparison with the later nanocomposite samples. Similar characterisation properties in the nanocomposite samples would indicate silver/silver halide nanoparticles forming within their matrix. This can confirm whether synthesis of matrix supported plasmonic photocatalyst nanocomposites has been achieved. 


\subsection{Silver/Silver Halide Wool Nanocomposites}

Following the experimental procedure described previously, $\mathrm{Ag} / \mathrm{AgX}$ wool nanocomposites were made with $\mathrm{AgCl}, \mathrm{AgBr}$ and $\mathrm{AgI} .{ }^{67}$ This was achieved using the method developed by Kelly to repeat her findings, and to compare the $\mathrm{Ag} / \mathrm{AgX}$ nanoparticles with the wool nanocomposites to see if what was observed was indeed the formation of $\mathrm{Ag} / \mathrm{AgX}$ particles on exposure to light. The $\mathrm{AgCl}$ and $\mathrm{AgBr}$ nanocomposites were found to be white when immediately synthesised, and developed a purple colour on light exposure. The white colour on first synthesis is to be expected, as both $\mathrm{AgCl}$ and $\mathrm{AgBr}$ are white in colouration as is the wool matrix itself. The UV/Vis spectra indicated that a broad visible region absorption had developed over the period of light exposure. (Figure $26 \& 27$ ) However there was no such absorption seen with AgI wool composites. The AgI wool composites developed a yellow colouration during synthesis, which was retained on light exposure. (Figure 28) This is due to $\mathrm{AgI}$ and its less photolytic nature than $\mathrm{AgBr}$ and $\mathrm{AgCl}$. The $\mathrm{AgI}$ wool composite also showed a large absorption from $<450 \mathrm{~nm}$, the typical absorption of AgI.

The $\mathrm{AgCl}$ and $\mathrm{AgBr}$ particles within the wool matrix absorb the light, and undergo selfsensitisation through photolysis. ${ }^{46,47}$ The silver halide nanoparticles ( $\mathrm{AgCl}$ and $\mathrm{AgBr}$ ) absorb the light they are exposed to after synthesis, and form the $\mathrm{Ag} / \mathrm{AgX}$ nanoparticles in situ within the wool matrix by partially reducing the $\mathrm{Ag}^{+}$to $\mathrm{Ag}^{0}$ and releasing small amounts of halides as their respective halogen gas. As with the $\mathrm{Ag} / \mathrm{AgCl}$ nanoparticles, the increased visible absorption that is seen to develop over the light exposure is due to the surface plasmon resonance of these newly formed $\mathrm{Ag}^{0}$ nanoregions on the surface of the silver halide nanoparticle. ${ }^{32,45} \mathrm{As}$ it can be seen below, the $\mathrm{AgCl}$ wool nanocomposite and $\mathrm{AgBr}$ wool nanocomposite develop a similar visible absorption. (Figure $26 \& 27$ ) However the $\mathrm{Ag} / \mathrm{AgBr}$ wool nanocomposite has a broader absorption across the visible region than the $\mathrm{Ag} / \mathrm{AgCl}$ wool nanocomposite. Also of note is the jagged absorption at around $350 \mathrm{~nm}$ wavelength, this is due to the lamp change of the Cary $100 \mathrm{UV}-\mathrm{V}$ is spectrophotometer. 


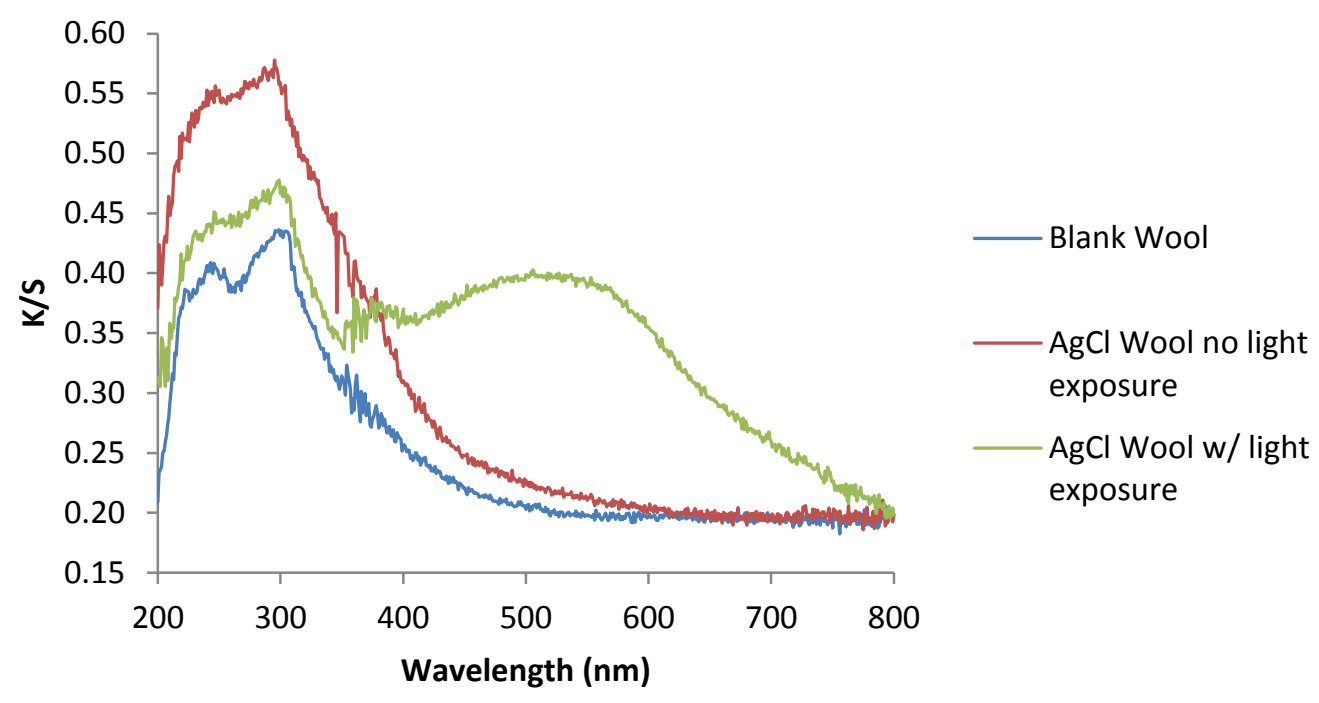

Figure $26 \mathrm{UV} /$ Vis Spectra of $\mathrm{AgCl}$ wool nanocomposite before and after light exposure

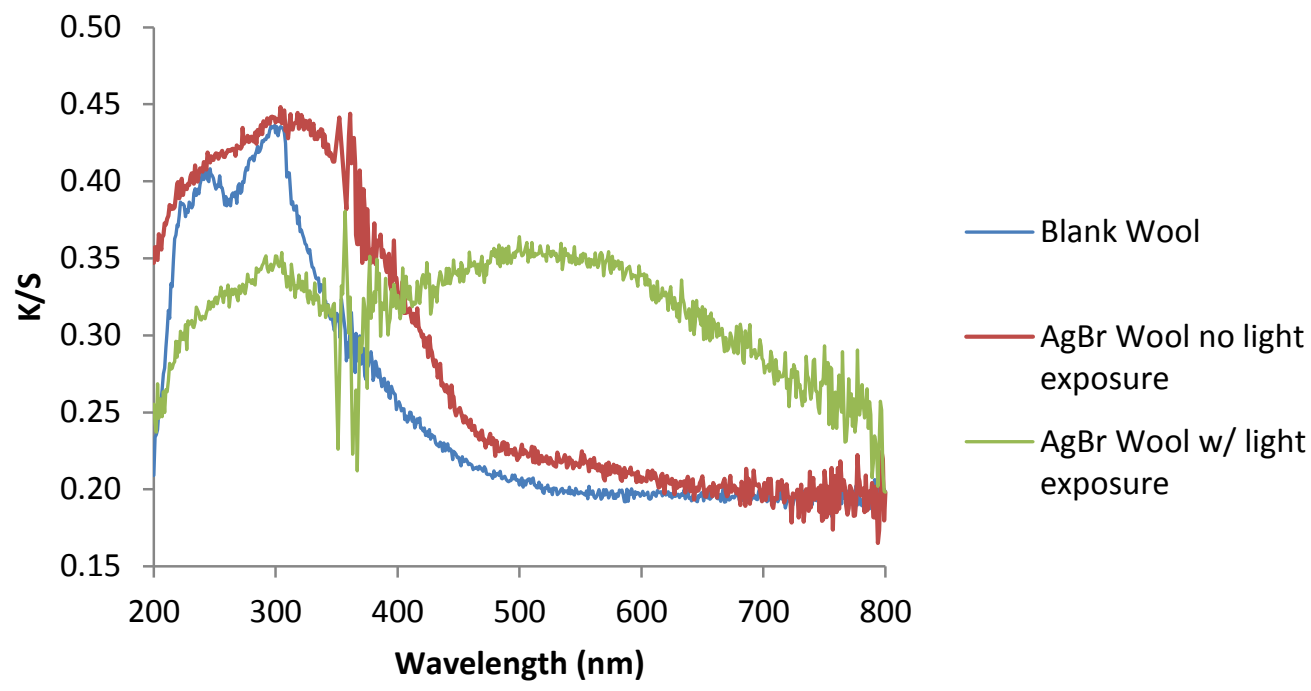

Figure $27 \mathrm{UV} / \mathrm{Vis}$ Spectra of $\mathrm{AgBr}$ wool nanocomposite before and after light exposure

As previously mentioned, the AgI wool nanocomposites appear to retain largely the same UV/Vis absorption before and after light exposure. (Figure 28) The silver iodide semiconductor conduction band absorption can be seen $<450 \mathrm{~nm}$, there is also some slightly increased absorption in the wavelength region of 450 to $500 \mathrm{~nm}$. This could be caused by very small amounts of $\mathrm{Ag}^{0}$ being formed from the $\mathrm{AgI}$ nanoparticles. ${ }^{49}$ The $\mathrm{AgI}$ has a lower photolysis rate, as discussed, hindering the formation of the $\mathrm{Ag}^{0}$ nanodomains. 


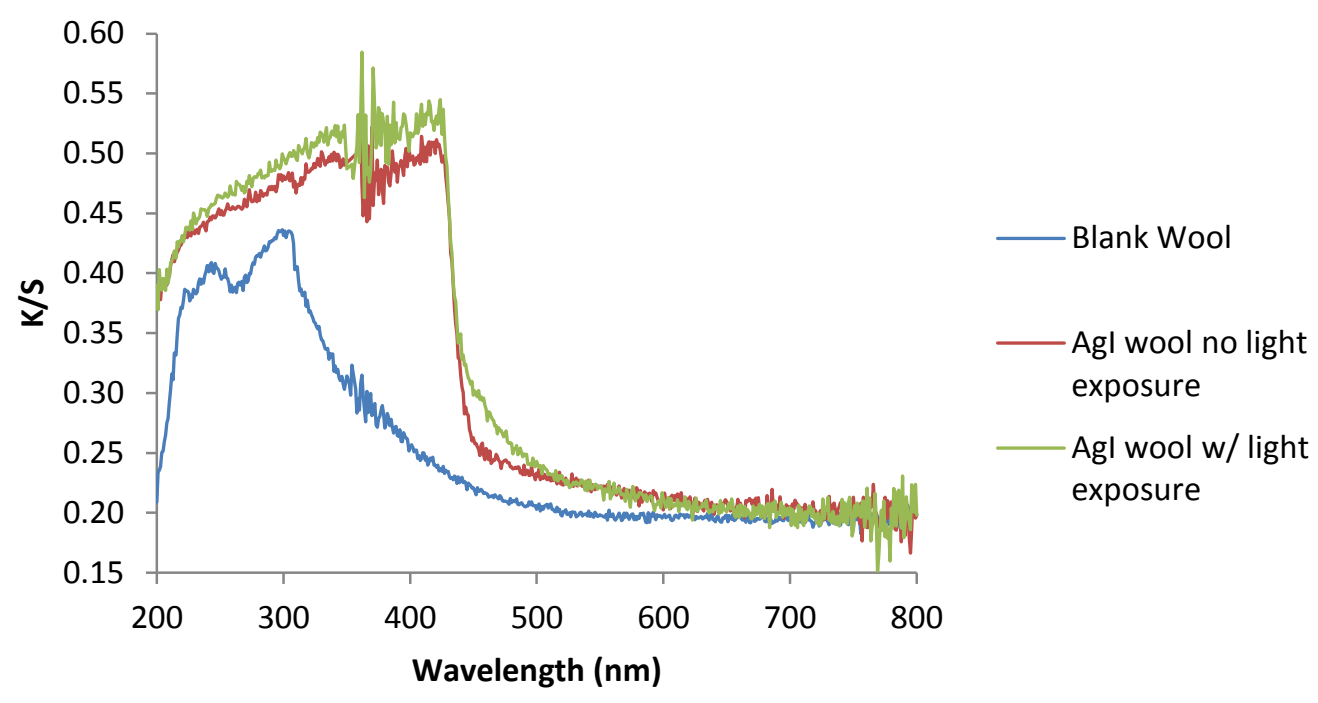

Figure $28 \mathrm{UV} /$ Vis Spectra of AgI wool nanocomposite before and after light exposure

XRD was used to confirm the presence of the silver halides within the wool fibre matrix. It was able to show that in all of the nanocomposites crystalline silver halides were present; this includes $\mathrm{AgCl}, \mathrm{AgBr}$ and $\mathrm{AgI}$. The $\mathrm{XRD}$ patterns for $\mathrm{AgCl}$ and $\mathrm{AgBr}$ are similar, due to their fcc cubic crystal structure and very similar lattice parameters. AgI on the other hand has a wurtzite crystal structure, giving a different XRD pattern. The flat mounting of the silver/silver halide wool nanocomposites and the sensitivity of the X'Pert Pro diffractometer made it possible to see the diffraction patterns of the crystalline silver halides, on top of the diffraction pattern of the blank wool matrix. (Figure 29)

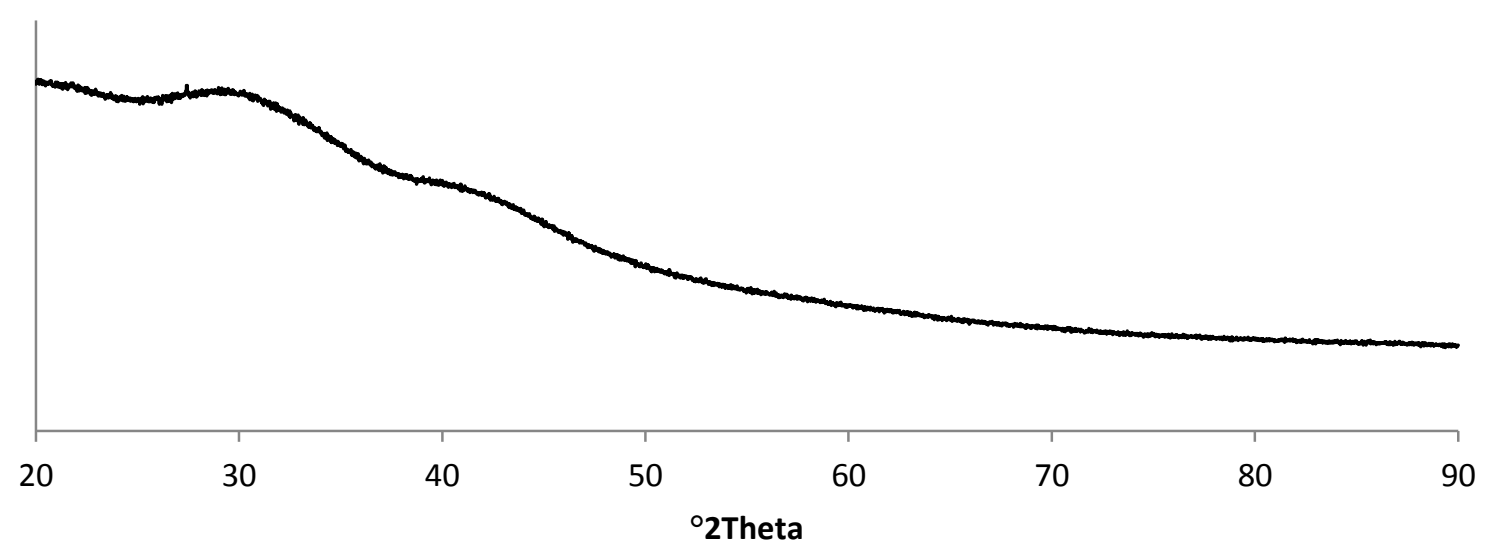


The XRD patterns gave confirmation that the silver halides were incorporated into the wool matrix during synthesis. It was also used to ascertain if light exposure did in fact cause photolysis of the silver halides, and form silver nanoregions on the surface of the silver halide nanoparticles within the nanocomposite. Diffraction patterns of composites exposed to light indicated that a silver 111 peak was beginning to develop, showing that small amounts of crystalline silver were developing within the nanocomposite over the period of light exposure. This Ag 111 peak shows that there is formation of silver/silver halide nanoparticles within the wool matrix of the nanocomposite. In order to confirm that an Ag 111 peak was in fact forming, increased Ag was used for a more concentrated sample, $300 \mathrm{ppm} \mathrm{Ag}$. This provided a more visible Ag 111 peak over the wool diffraction pattern. (Figure $30 \& 31$ ) Original XRD patterns for $\mathrm{Ag} / \mathrm{AgCl}$ wool nanocomposites with lower $\mathrm{Ag}$ concentration can be seen in the appendix.

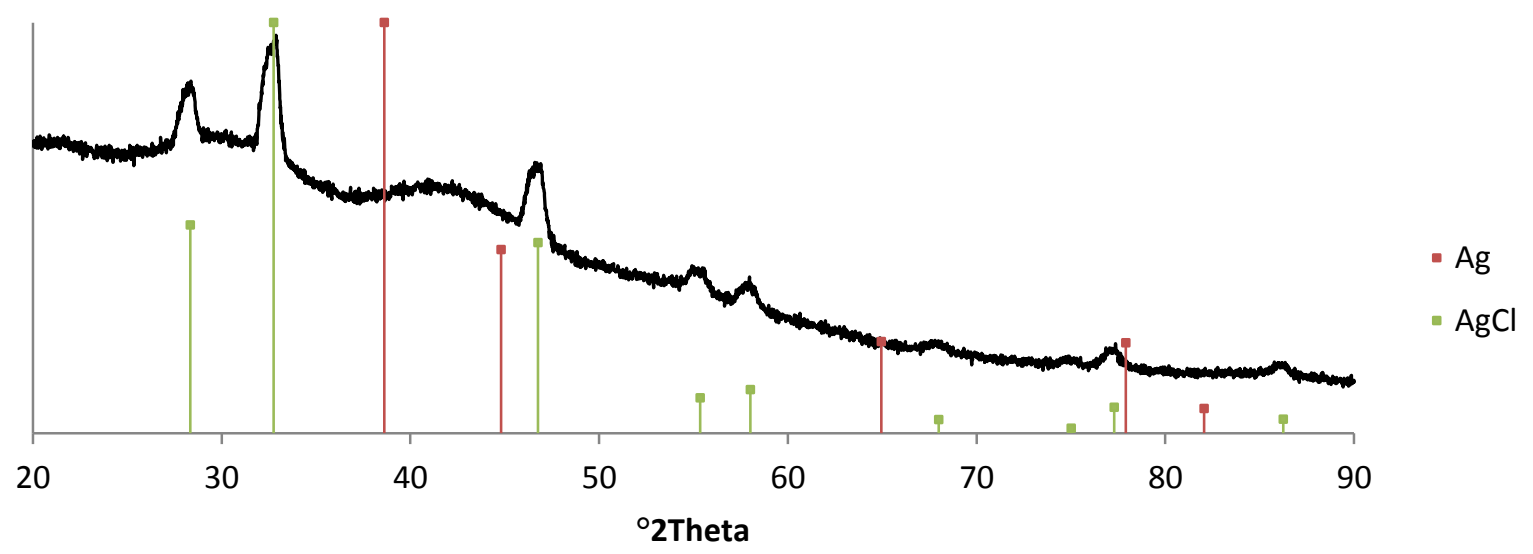

Figure 30 XRD pattern of $\mathrm{AgCl}$ Wool Nanocomposite without light exposure 


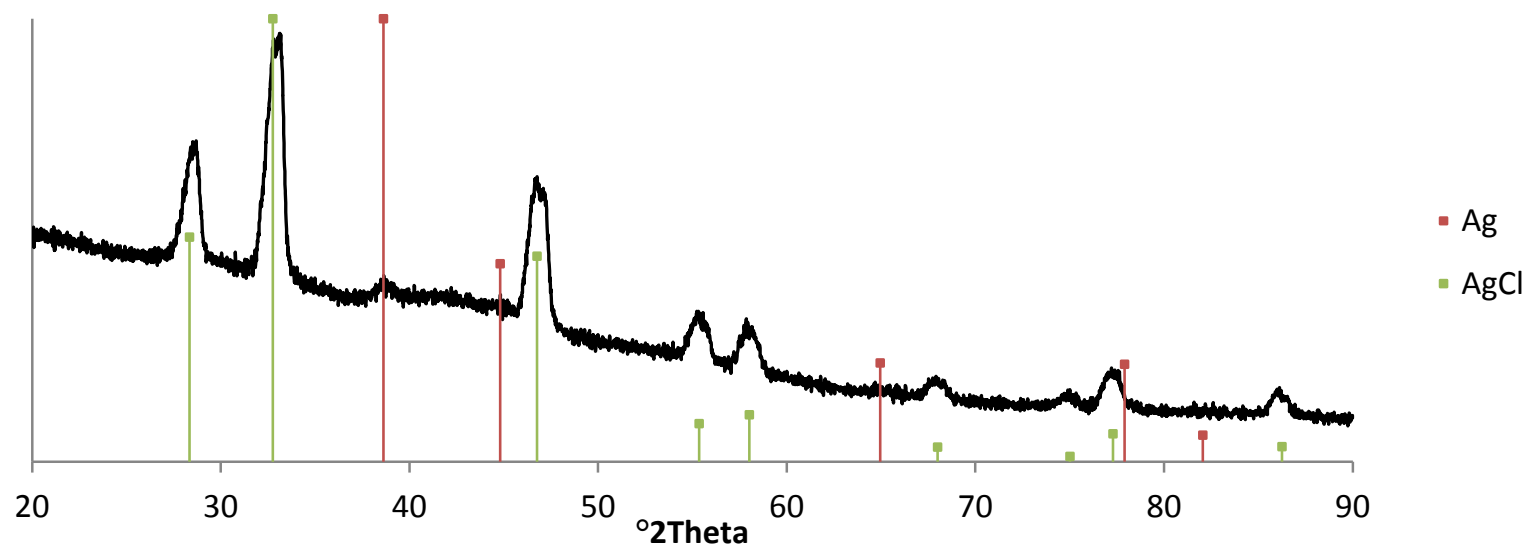

Figure 31 XRD pattern of $\mathrm{AgCl}$ Wool Nanocomposite after light exposure

The $\mathrm{Ag} / \mathrm{AgBr}$ wool nanocomposite XRD patterns before and after light exposure do not show an as intense peak for the $\mathrm{Ag} 111$ as for the $\mathrm{AgCl}$ wool sample, as lower $\mathrm{Ag}$ concentration was used in the sample preparation. However, it shows a small Ag 111 peak. This can be seen after light exposure, through a change in the profile of the background. (Figure 33) The AgI wool nanocomposite XRD pattern does not appear to have any formation of an Ag 111 peak, although this would be expected to be only a slight peak due to the low level of photolysis. Also, the AgI diffraction pattern would make it difficult to determine if any crystalline $\mathrm{Ag}$ is formed. As the Ag 111 and the AgI 220 lie in close proximity to each other, allowing the AgI 220 to potentially swamp the Ag 111. (Figure 35)

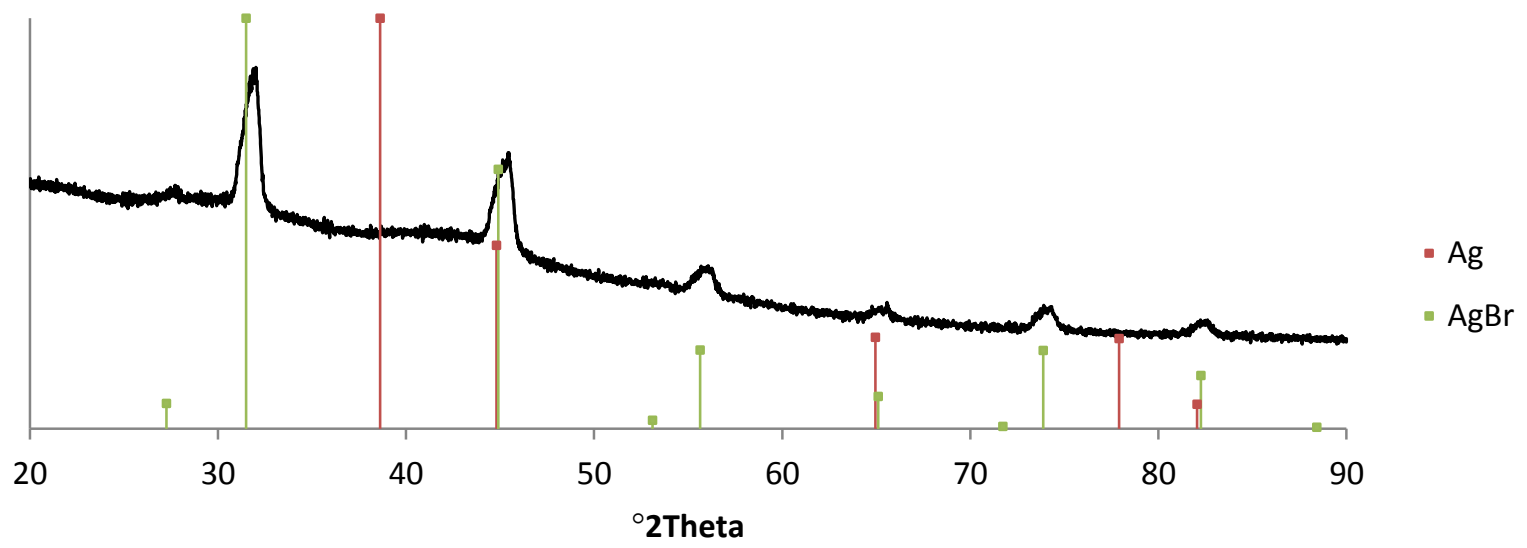

Figure 32 XRD pattern of $\mathrm{AgBr}$ Wool Nanocomposite without light exposure 


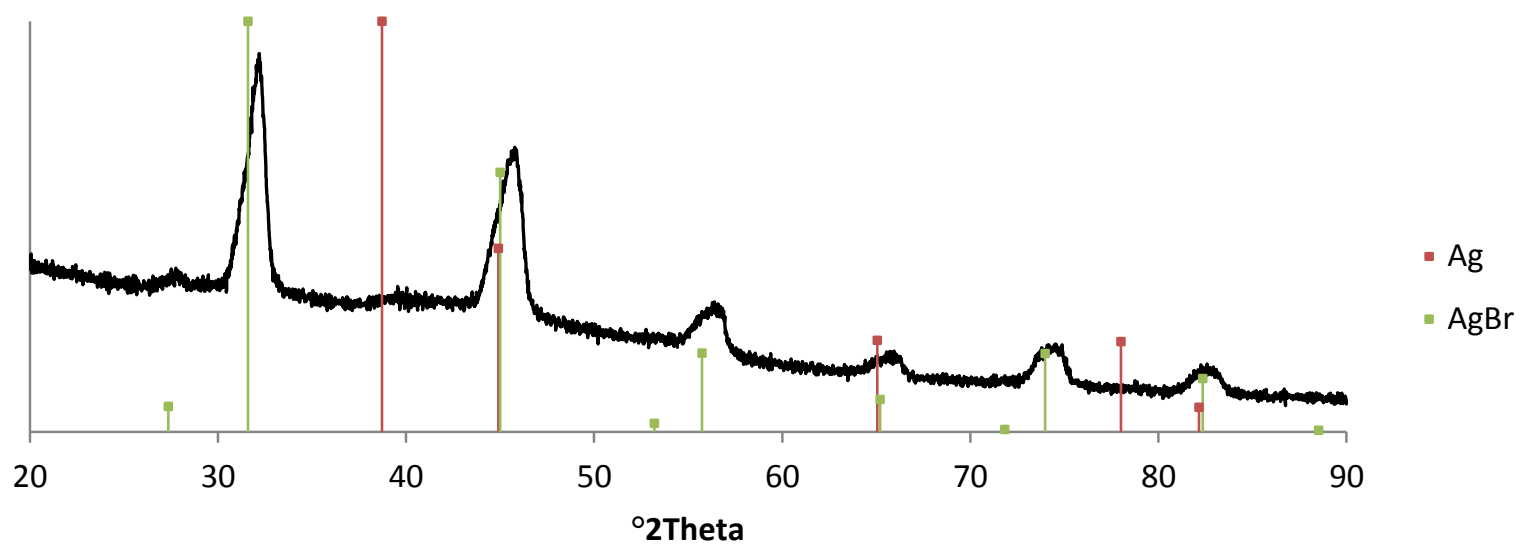

Figure 33 XRD pattern of $\mathrm{AgBr}$ Wool Nanocomposite after light exposure

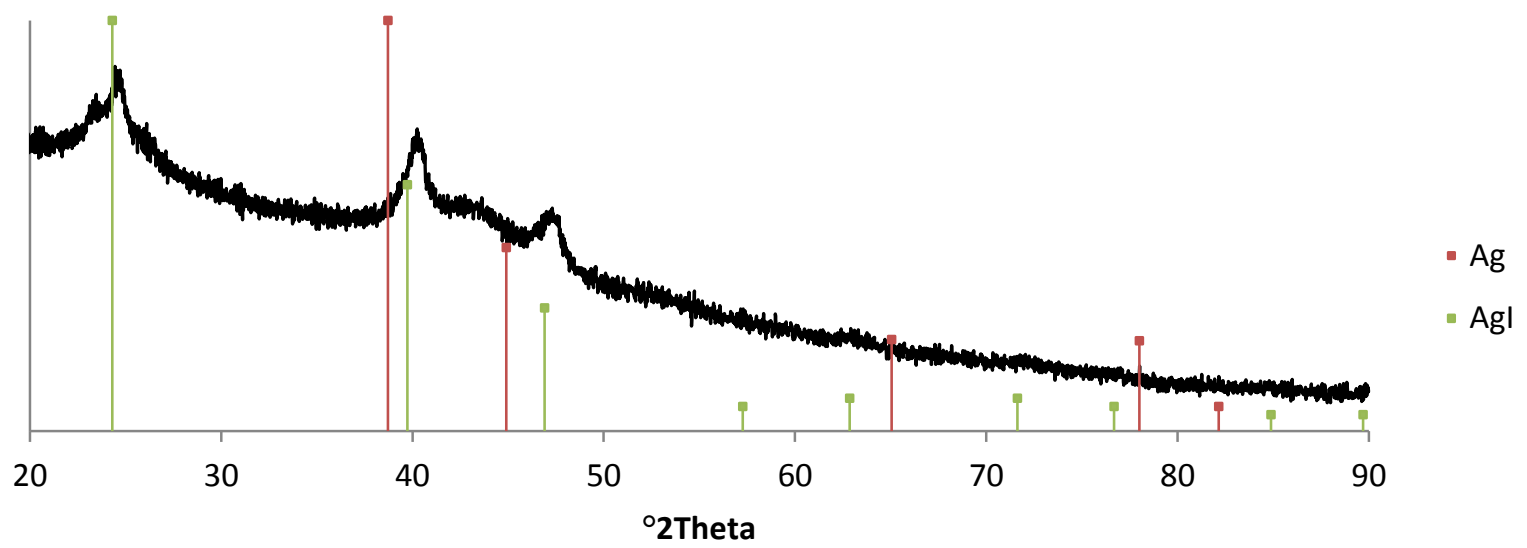

Figure 34 XRD pattern of AgI wool nanocomposite without light exposure

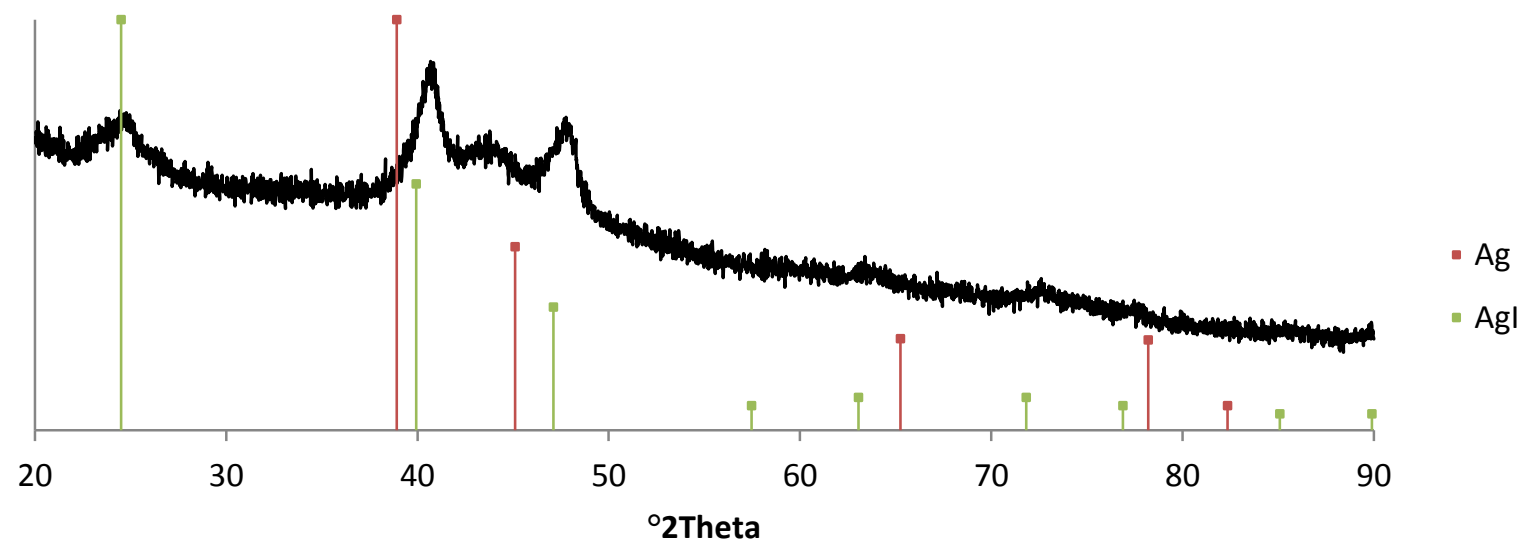

Figure 35 XRD pattern of AgI wool nanocomposite after light exposure 
The silver/silver halide nanoparticles formation is a self-sensitisation process, rather than just complete photolysis, when exposed to light. ${ }^{46,47}$ To show this to be the case with periods the $\mathrm{Ag} / \mathrm{AgCl}$ wool nanocomposite was subjected to a period of extended light exposure, under the Xe arc lamp (up to 5 days). There was no significant increase in the Ag 111 peak and no significant decrease in intensity of the peak in the silver halide diffraction patterns. (Figure 36 \& 37) The amount of self-sensitisation is low, giving a small Ag 111 peak. In the case of most low concentration Ag samples, almost no peak was visible. Often a small bump in the background diffraction pattern was observed, so again a more concentrated $300 \mathrm{ppm} \mathrm{Ag}$ $\mathrm{AgCl}$ wool nanocomposite sample was used to identify any changes.

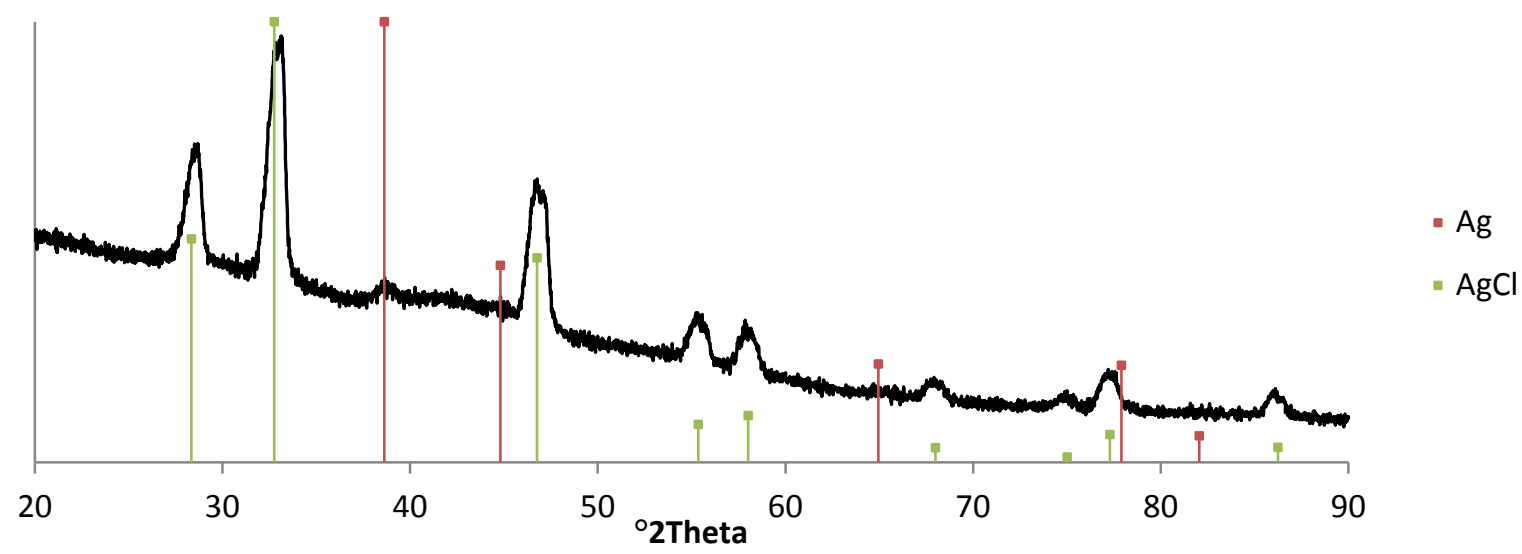

Figure 36 XRD pattern of $\mathrm{AgCl}$ wool nanocomposite after light exposure

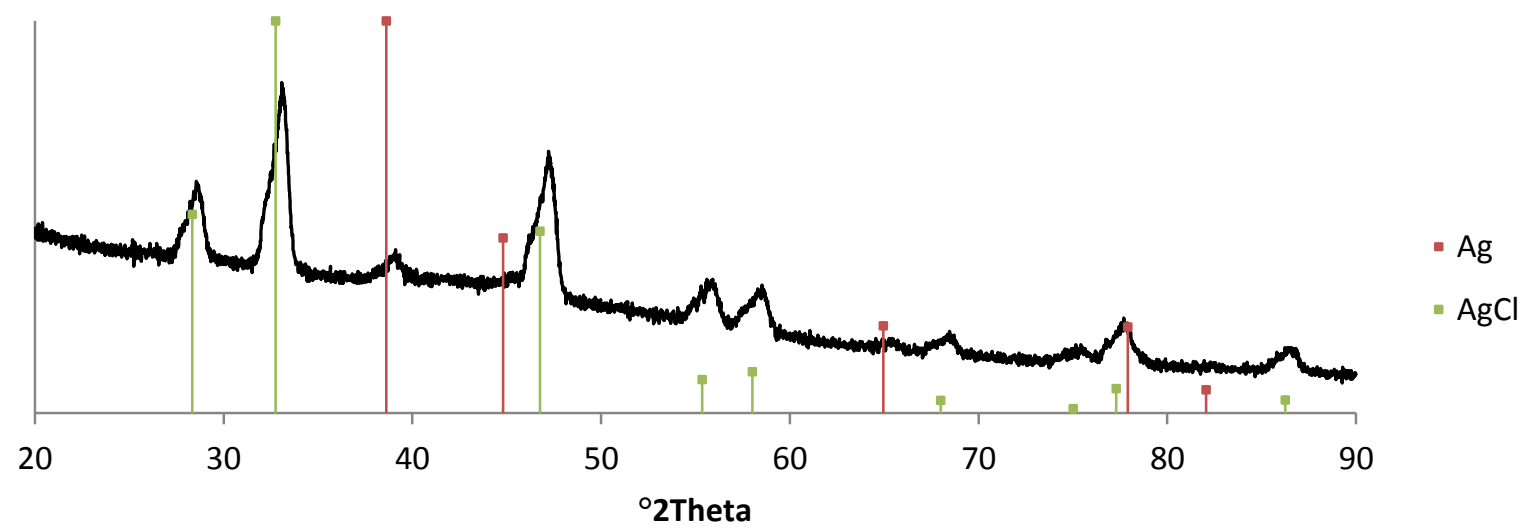

Figure 37 XRD pattern of $\mathrm{AgCl}$ wool nanocomposite after 5 days light exposure in Xe Arc lamp 
SEM was used as a characterisation technique to view the morphology of the silver/silver halide wool nanocomposites and the distribution of the nanoparticles within the wool matrix. A first look at the SEM images shows that in all cases for $\mathrm{AgCl}, \mathrm{AgBr}$ and $\mathrm{AgI}$ there are nanoparticles present within the wool fibre matrix. The nanoparticles can be seen in the backscatter SEM images, where the silver halide nanoparticles are indicated as bright areas. Backscatter images emphasise elements of high atomic number, giving better contrast. In all of the $\mathrm{AgCl}, \mathrm{AgBr}$ and $\mathrm{AgI}$ nanocomposites there is a distribution of particles across the fibre. However, AgI wool nanocomposite appears to have a more even spread of particles across the fibre, compared to the $\mathrm{AgCl}$ and $\mathrm{AgBr}$ analogues. The secondary electron images (SEI) of the silver/silver halide wool nanocomposites indicate that the silver/silver halide nanoparticles are below the surface of the fibre, with few or no particles on the surface. This is indicated through the relatively smooth surfaces of the fibres seen in the SEI images, which give a better view of the morphology. Typically there would be large amounts of surface agglomerates if the nanoparticles were deposited on the fibre, especially in the cuticle edges which are higher in surface energy. ${ }^{67,78}$ These images indicate that the particles are formed within the fibre, and not simply deposited on or adhered to the surface of the fibre.
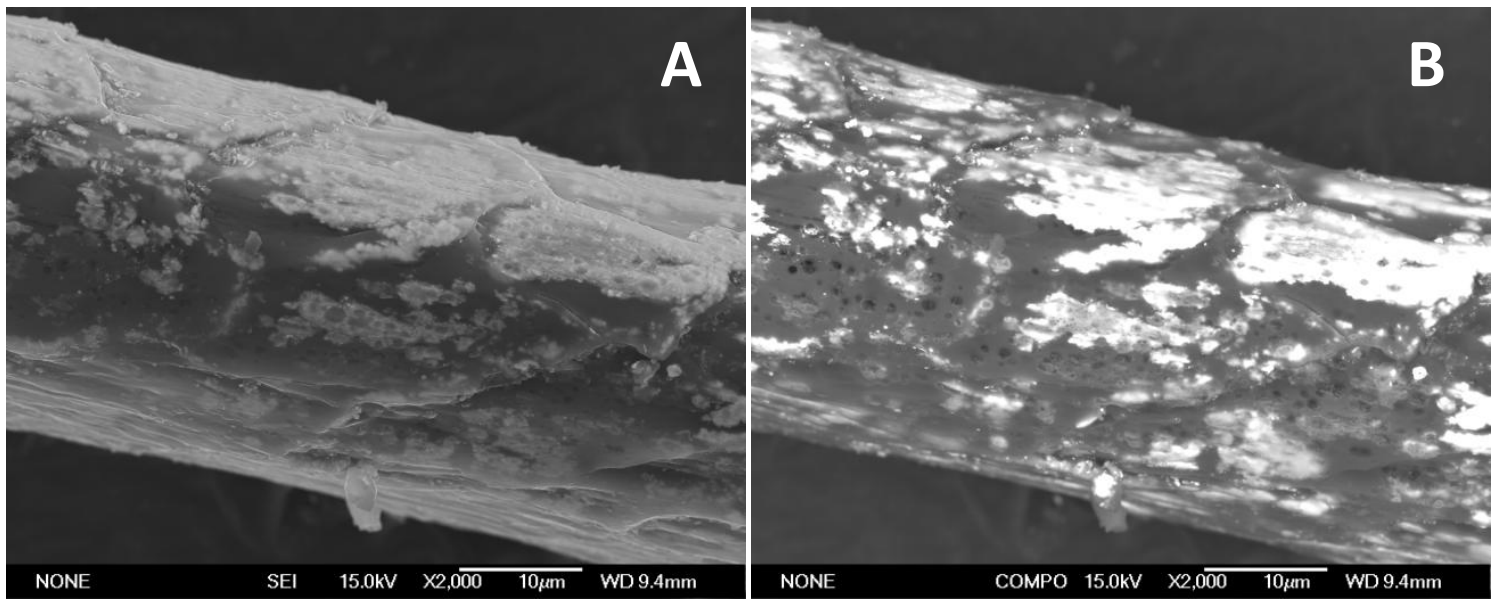

Figure 38 SEM images of $\mathrm{AgCl}$ wool nanocomposite A) SEI image B) Backscatter image 

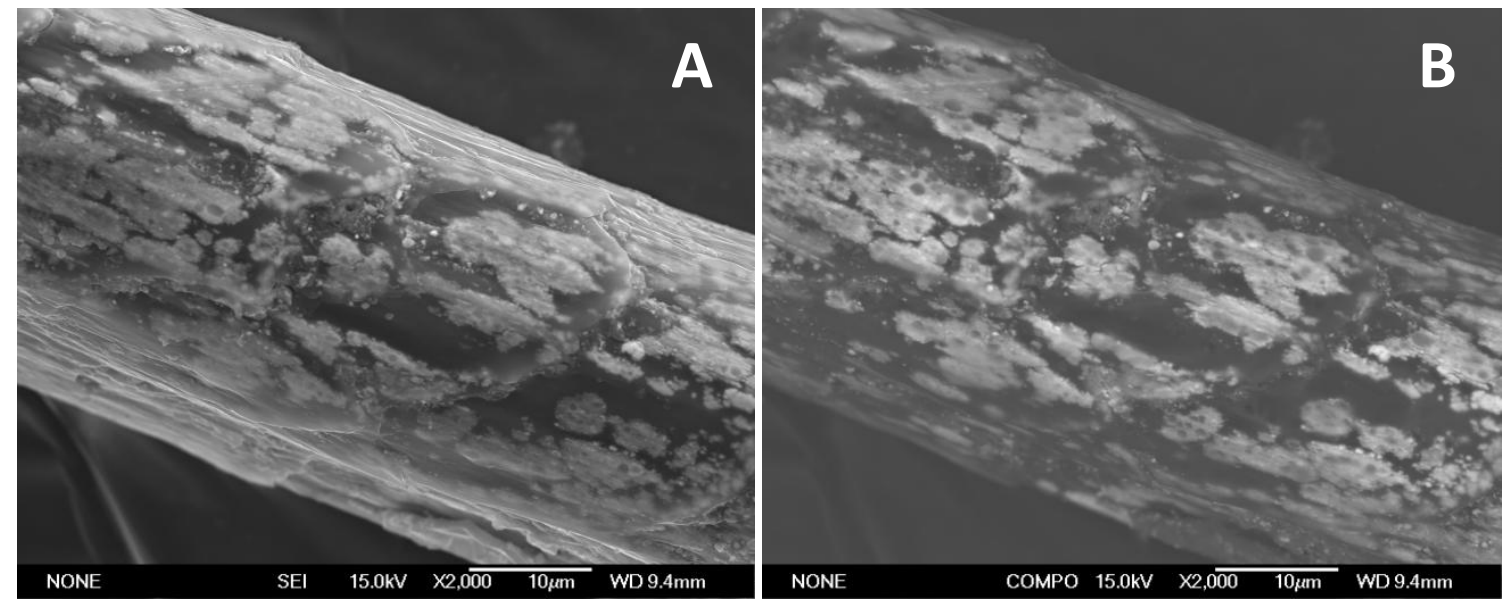

Figure 39 SEM images of AgBr wool nanocomposite A) SEI image B) Backscatter image
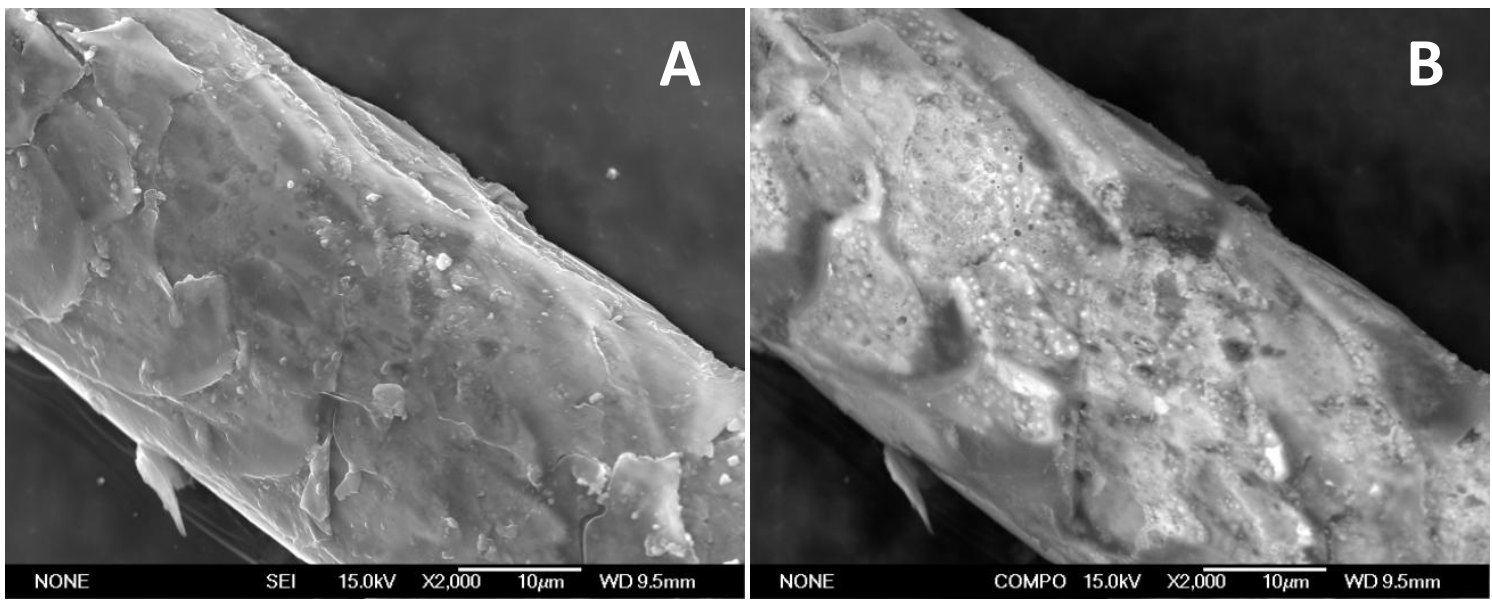

Figure 40 SEM Images of AgI wool nanocomposite A) SEl image B) Backscatter image

EDS analysis was carried out on the silver/silver halide wool nanocomposites for further confirmation of the silver halide particle formation within the nanocomposite matrix. EDS shows that silver and the respective halides were present within the composites. The EDS mapping also shows areas of high silver and halide content over lapping well, further showing that the particles seen are in fact silver halide particles within the wool fibres. $\mathrm{AgCl}$ and $\mathrm{AgBr}$ wool nanocomposites (Figure $41 \& 42$ ) show a clumped distribution throughout the fibres, with large agglomerates in places whilst sill having substantial coverage across the whole fibre. $\mathrm{AgI}$ wool nanocomposite samples appear to be more disperse than the $\mathrm{AgCl}$ and $\mathrm{AgBr}$ samples, with less aggregates and a better fibre coverage. (Figure 43) 


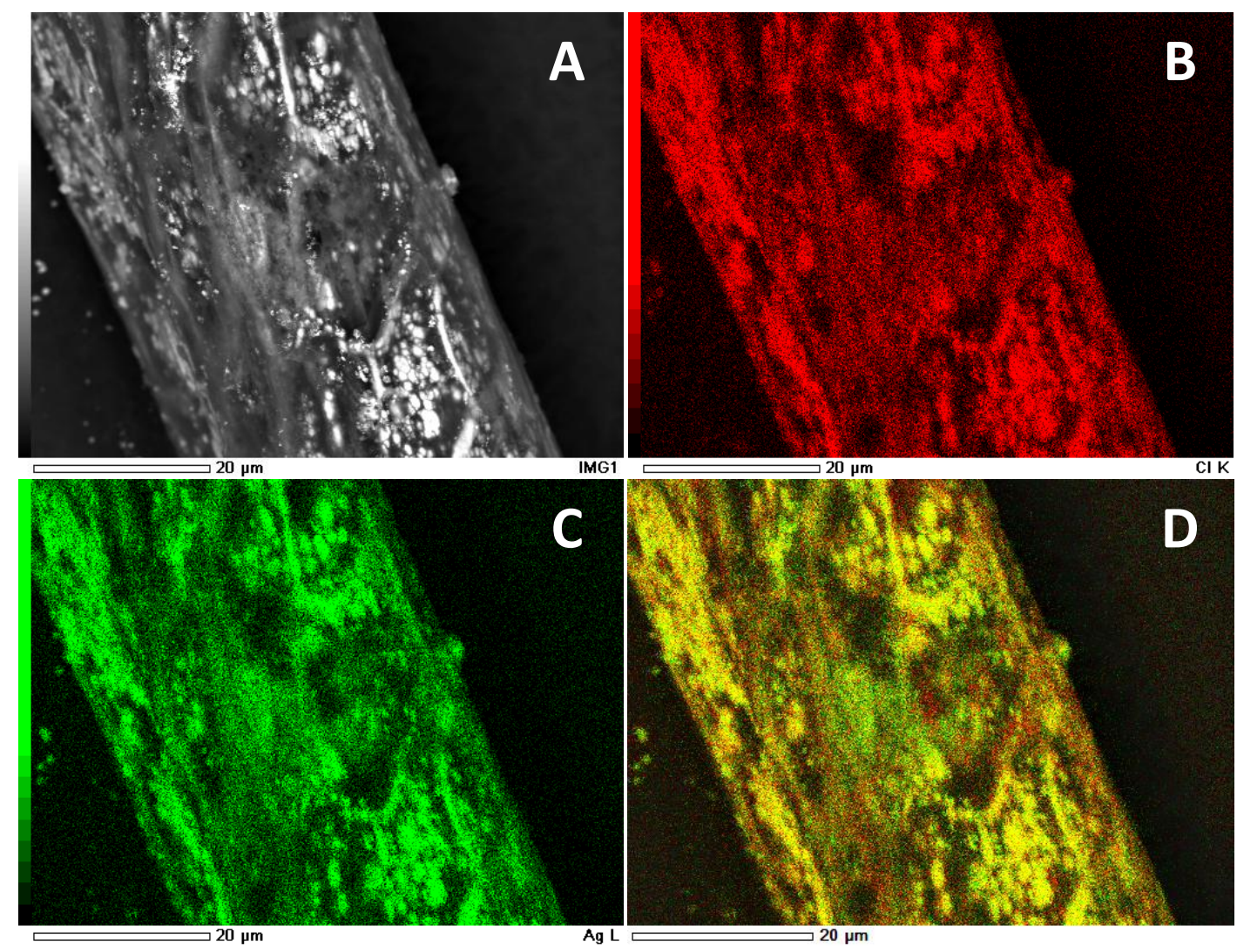

Figure $41 \mathrm{AgCl}$ wool nanocomposite A) Backscatter SEM image B) EDS map of Cl C) EDS map of Ag D) EDS overlay
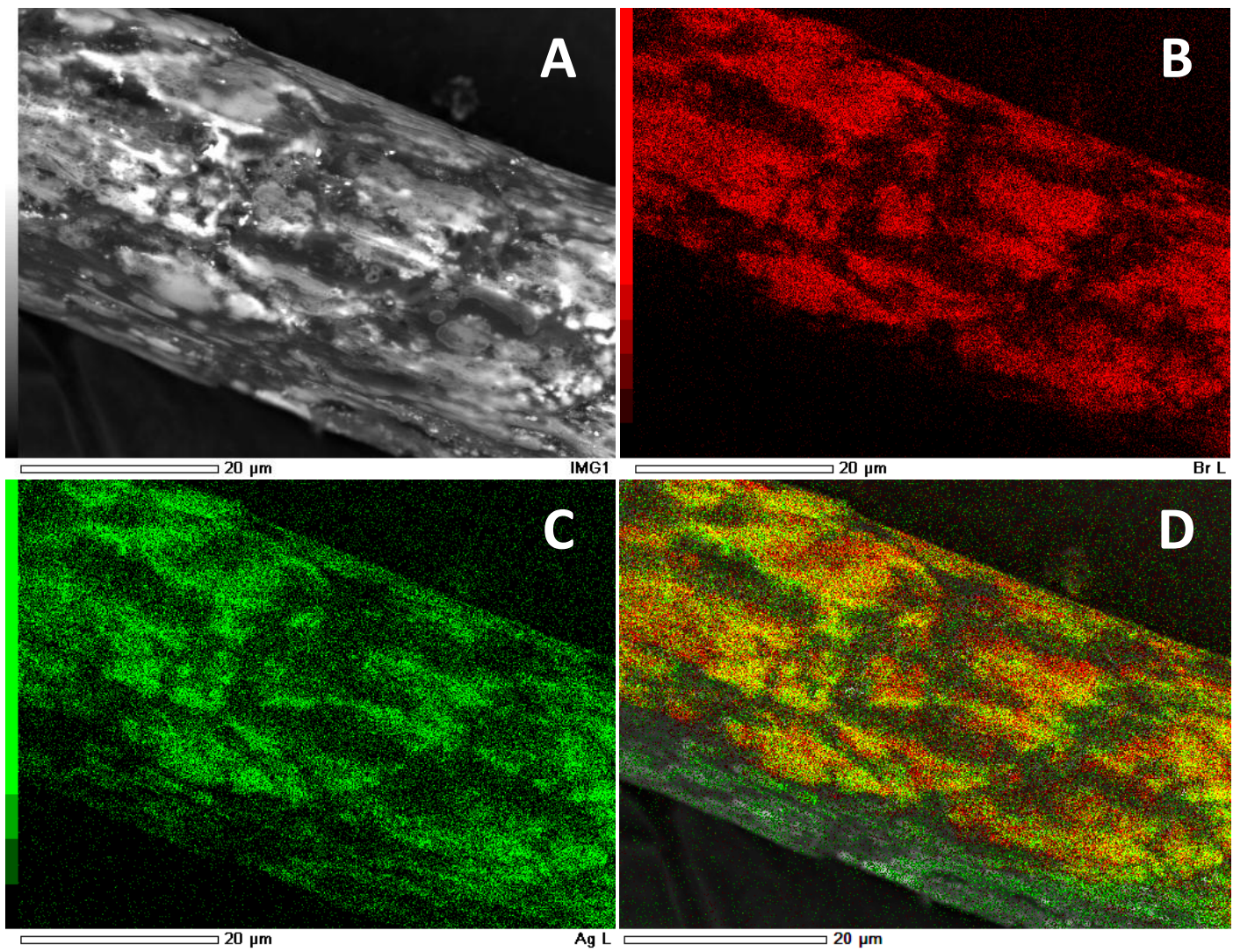

Figure $42 \mathrm{AgBr}$ wool nanocomposite A) SEM backscatter image B) EDS map of $\mathrm{Br}$ C) EDS map of $\mathrm{Ag}$ D) EDS overlay 


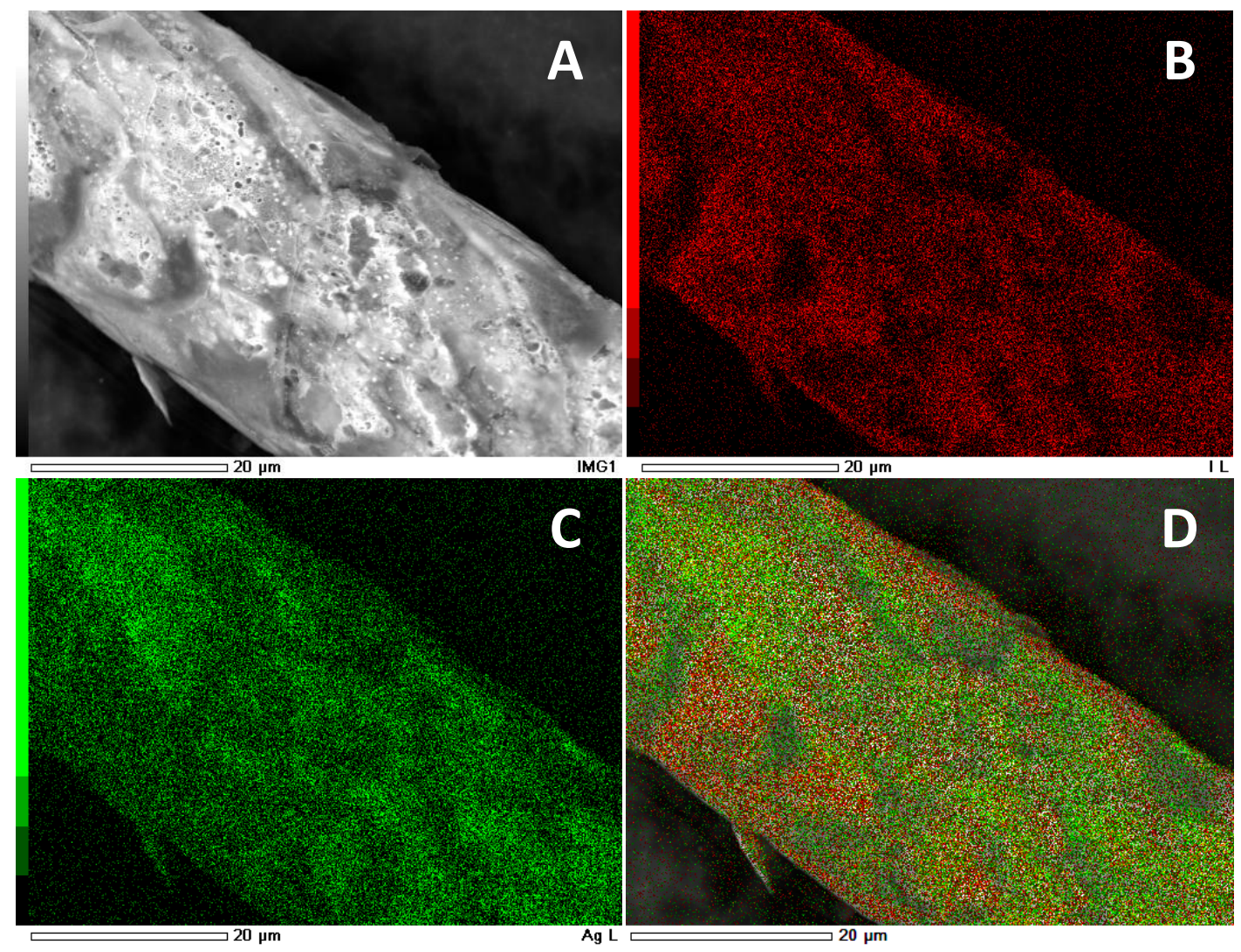

Figure 43 AgI wool nanocomposite A) SEM backscatter image B) EDS map of I C) EDS map of Ag D) EDS overlay

Cross-sectional SEM was also carried out on the silver/silver halide wool nanocomposites. This gave a good indication of the distribution of the silver halide nanoparticles throughout the centre of the fibre. It was seen that the silver halide nanoparticles were found just below the surface. The nanoparticles formed in a ring distribution below the surface, and had no particles distributed towards the centre of the fibre. This formation of silver halide nanoparticles just below the cuticles of the wool fibre was observed in all the silver/silver halide wool nanocomposites. $\mathrm{AgCl}$ and $\mathrm{AgBr}$ wool nanocomposites clearly show this distribution in the SEM images with EDS overlay seen below in figure $44 \& 45$. However, cross sectional EDS of the AgI wool nanocomposite was not obtained due to EDS sensitivity issues. The backscatter SEM image does however shows that the AgI wool nanocomposite (Figure 46) does also have nanoparticles formed below the fibre surface as a ring, in concurrence with the $\mathrm{AgCl}$ and $\mathrm{AgBr}$ wool nanocomposites. 


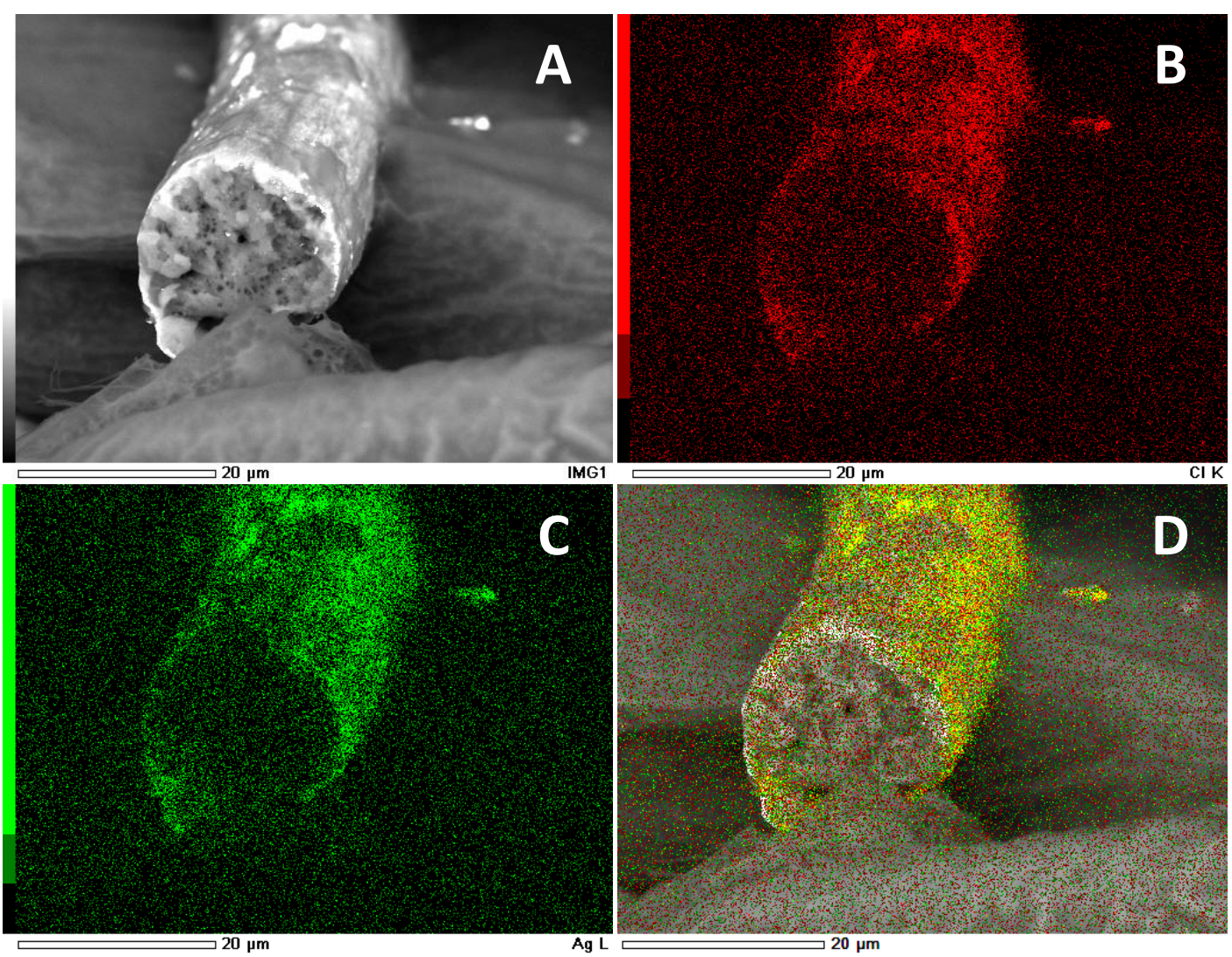

Figure $44 \mathrm{AgCl}$ wool cross section A) SEM backscatter image B) EDS map of Cl C) EDS map of $\mathrm{Ag}$ D) EDS overlay
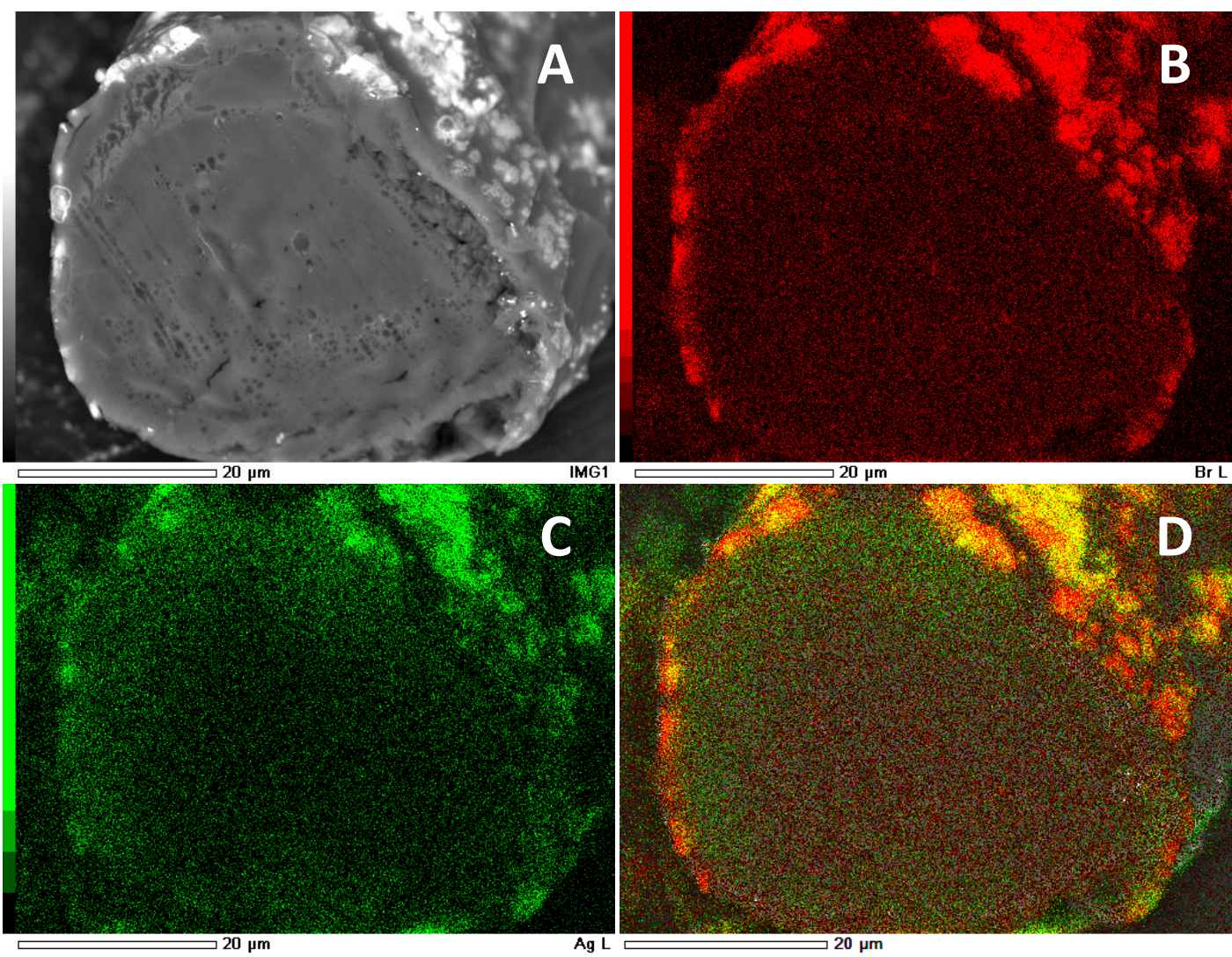

C.

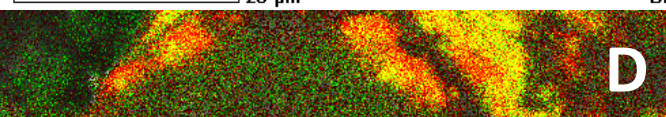

Figure $45 \mathrm{AgBr}$ wool cross section A) SEM backscatter image B) EDS map of $\mathrm{Br}$ C) EDS map of $\mathrm{Ag}$ D) EDS overlay 

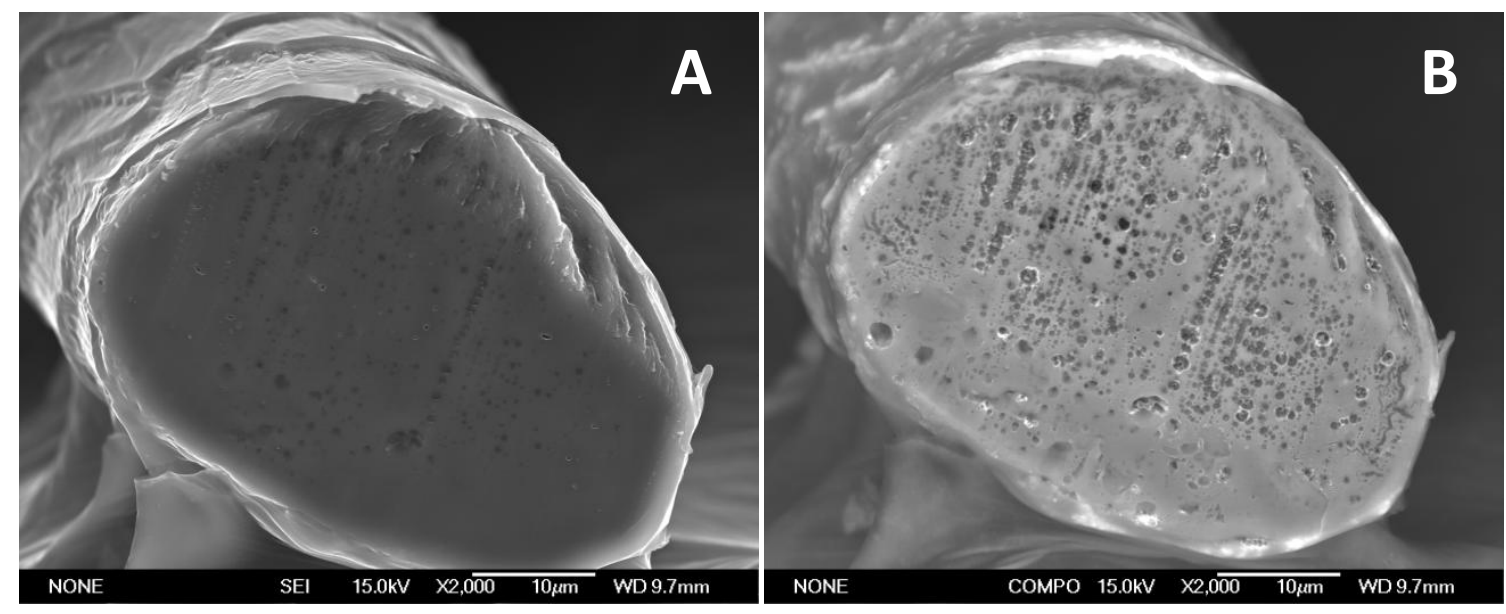

Figure 46 AgI wool nanocomposite cross section A) SEl image B) Backscatter image

The mechanism of particle formation within the fibre is believed to be one of precipitation, with size control and stabilisation of the particles being mediated by the wool fibre itself. The doped fibres have halide ions distributed throughout the wool fibre. With washing, the surface halide ions are removed from the fibre leaving only halide ions beneath the surface and in the bulk of the fibre. Upon immersion in the silver nitrate solution the $\mathrm{Ag}^{+}$ions absorbed by the wool fibre and remain under the fibre surface. The halide ions then migrate towards the $\mathrm{Ag}^{+}$ions within the fibre. When the ions meet, the silver halide is formed, all of the silver halides are very insoluble within the aqueous environment in which synthesis takes place. $^{67}$

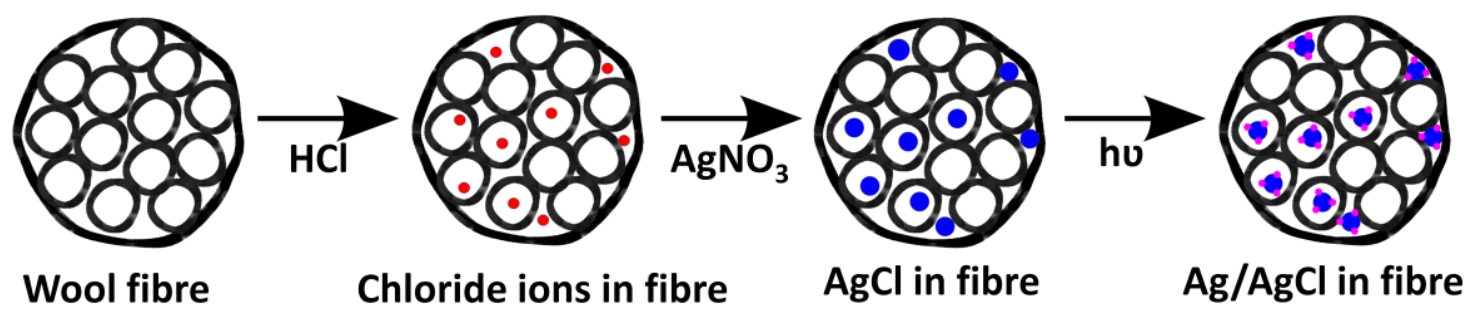

Figure 47 Representation of the formation mechanism of the $\mathrm{Ag} / \mathrm{AgCl}$ wool nanocomposite

This method of particle formation means that the mobile halide ions migrate towards the $\mathrm{Ag}^{+}$ ions in solution as they are taken up. This is the reason why the nanoparticles form just below the surface of the wool fibre and not towards the fibre centre. 
In summary the synthesis methods described in the earlier experimental section have been shown to produce silver/silver halide nanocomposites of $\mathrm{AgCl}, \mathrm{AgBr}$ and $\mathrm{AgI}$. XRD and EDS analysis have shown that silver/silver halide nanoparticles are formed within the wool fibre matrix. SEM has also shown that these nanoparticles form within the fibre, just below the fibre surface under the cuticles. UV/Vis and XRD have shown that upon light exposure the silver halide nanoparticles undergo photochemical reaction, developing visible region absorption and crystalline silver. These are characteristic of the silver/silver halide nanoparticles used as plasmonic photocatalysts. Hence, silver/silver halide wool nanocomposites have been produced with the potential as matrix stabilised plasmonic photocatalysts. These have the potential for photocatalytic applications in waste water treatment or air filter systems where matrix stabilised photocatalysts can be used in dual contaminant breakdown and filtration role.

\subsection{Silver/Silver Halide Kraft Fibre Nanocomposites}

Through modifying the experimental procedure for synthesising silver/silver halide wool nanocomposites, an experimental method for silver/silver halide kraft fibre nanocomposites was designed. Using this method, which is described previously in the experimental section, silver/silver halide kraft fibre nanocomposites were made. Using both bleached and unbleached kraft fibres, nanocomposites using $\mathrm{AgCl}, \mathrm{AgBr}$ and $\mathrm{AgI}$ were prepared. Kraft fibres were explored in order to see if other matrices could be used as a support for silver/silver halide nanoparticles in addition to wool. Using both bleached and unbleached kraft fibres, where bleached fibres have no lignin and no ability for reduction and formation of nanoparticles, it could also be determined whether fibre functionality had any effect on silver/silver halide nanoparticle formation during synthesis. ${ }^{68}$

As seen with the silver/silver halide wool nanocomposites, the observed colour of the samples changed with exposure to light in the case of the $\mathrm{AgCl}$ and $\mathrm{AgBr}$ based nanocomposites. This was very noticeable in the bleached kraft samples as it was a major 
departure from the previously white colour of the fibres. The colour change was more difficult to see with unbleached kraft fibres, which have a naturally dark brown colouration. The UV/Vis spectra of the unbleached kraft fibre nanocomposites show that there is the development of a broad visible region absorption in the $\mathrm{AgCl}$ (Figure 48) and $\mathrm{AgBr}$ (Figure 49) nanocomposites, when they are exposed to light. AgI based unbleached kraft fibre nanocomposites (Figure 50) have the typical AgI absorption from $<450 \mathrm{~nm}$ as seen in the AgI wool nanocomposite. In the UV region of the unbleached kraft fibre spectra, there is a large absorption seen due to the chromophores in lignin. This creates a very different absorption profile to the bleached kraft fibre nanocomposites.

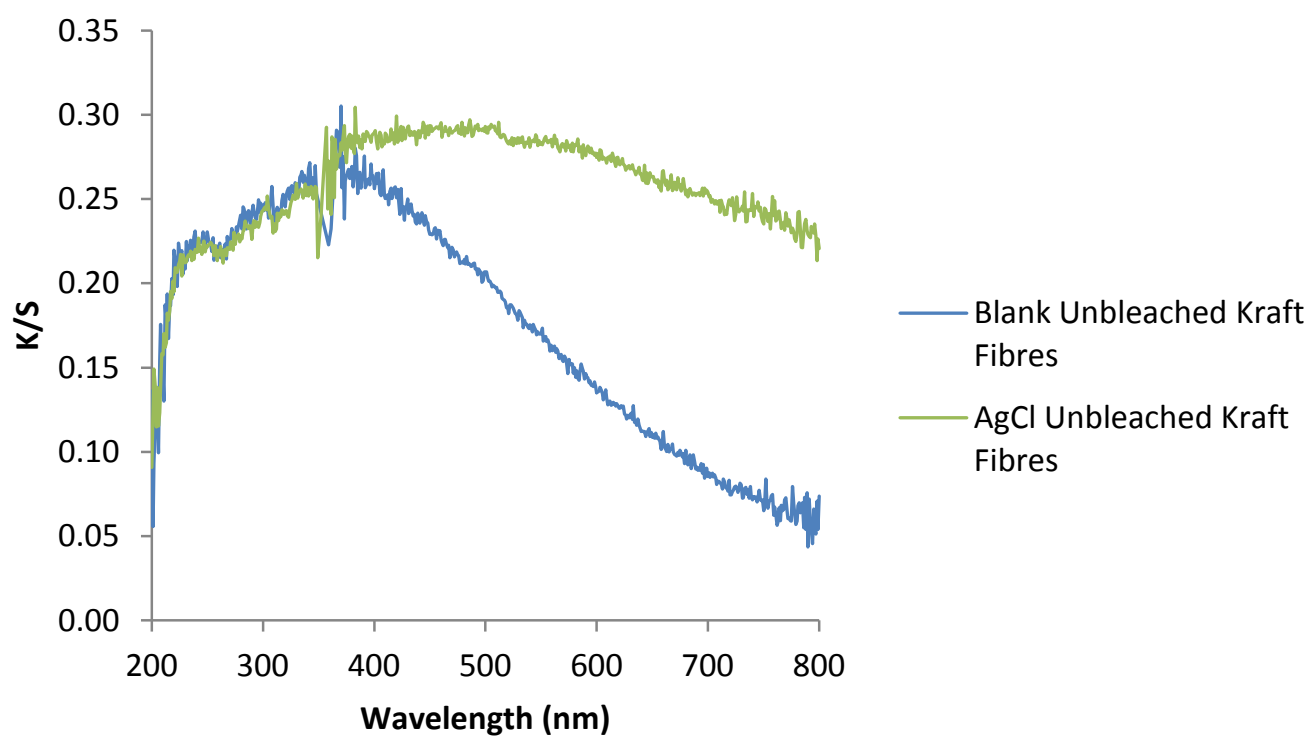

Figure $48 \mathrm{UV} / \mathrm{Vis}$ of $\mathrm{AgCl}$ unbleached kraft fibres after light exposure 


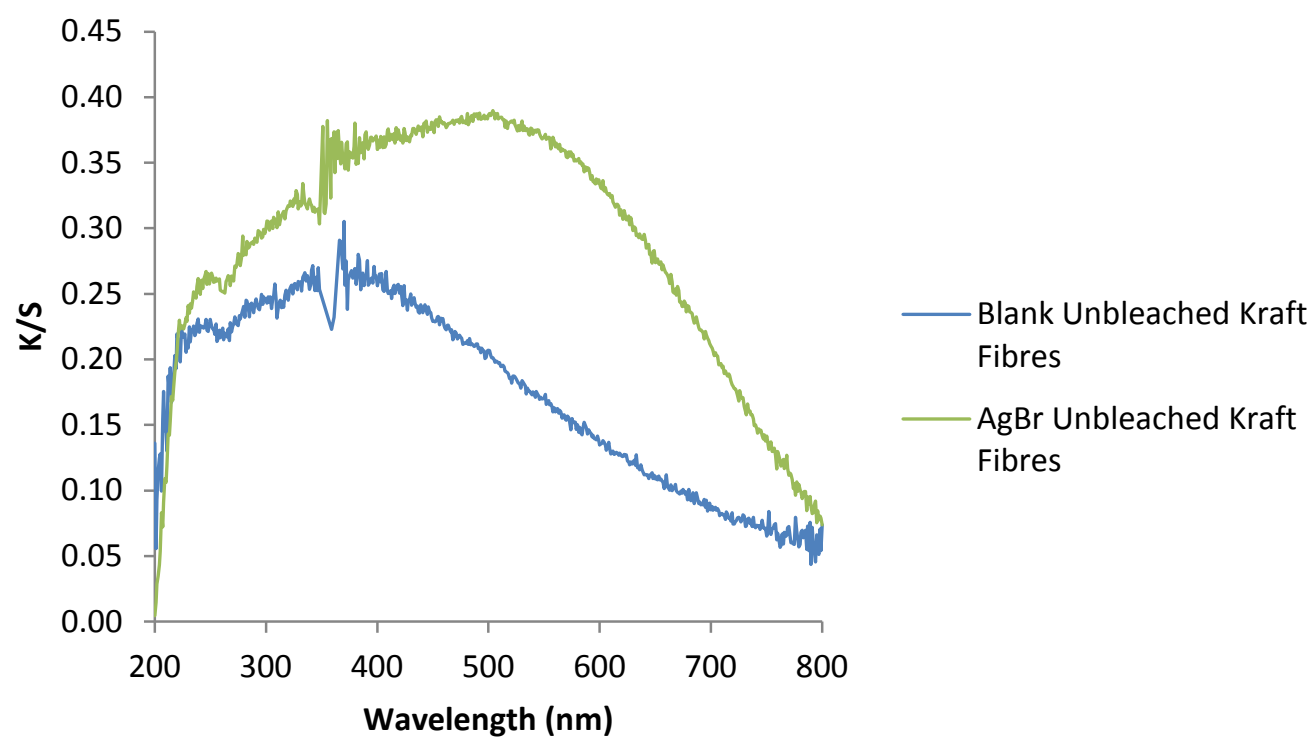

Figure $49 \mathrm{UV} / \mathrm{Vis}$ of $\mathrm{AgBr}$ unbleached kraft fibres with light exposure

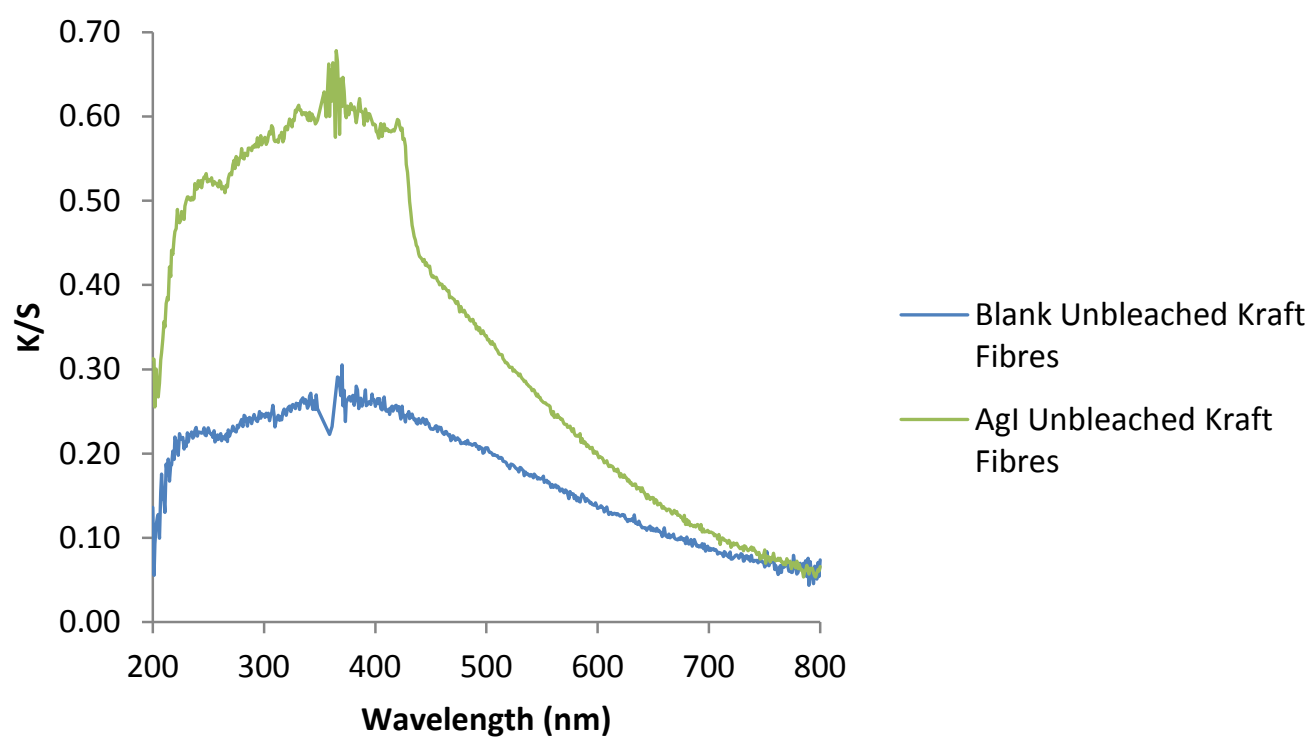

Figure $50 \mathrm{UV} / \mathrm{Vis}$ of $\mathrm{Agl}$ unbleached kraft fibres with light exposure

The bleached kraft fibre nanocomposites showed a similar trend to that of the unbleached kraft fibre nanocomposites in the visible region of the UV/Vis spectra. $\mathrm{AgCl}$ and $\mathrm{AgBr}$ based bleached kraft nanocomposites show strong broad visible absorptions that developed after light exposure as seen in figure $51 \& 52$. This absorption is very similar to that seen in the 
$\mathrm{Ag} / \mathrm{AgCl}(\mathrm{Br})$ wool nanocomposites, and so as expected also have similar purple coloured fibres. The AgI bleached kraft nanocomposite is a bright yellow colour, again similar to the AgI wool nanocomposite. The AgI bleached kraft nanocomposite displays the typical AgI absorption spectra with the conduction band absorbance of $<450 \mathrm{~nm}$, although it has an increase in absorption in the wavelength region of 450-600 nm, similar to AgI wool with light exposure. (Figure 53) The development of this visible absorption on light exposure indicates that, regardless of fibre matrix, the AgX nanoparticles undergo the same formation of $\mathrm{Ag}^{0}$ and self-sensitisation with light exposure. The same visible region absorptions have been seen to develop whether wool, bleached or unbleached kraft, showing that the functionality within the fibre is not required for formation of the silver/silver halide nanoparticles. Also showing the precipitation formation mechanism suggested previously is indeed a good representation of the synthesis of the nanocomposites.

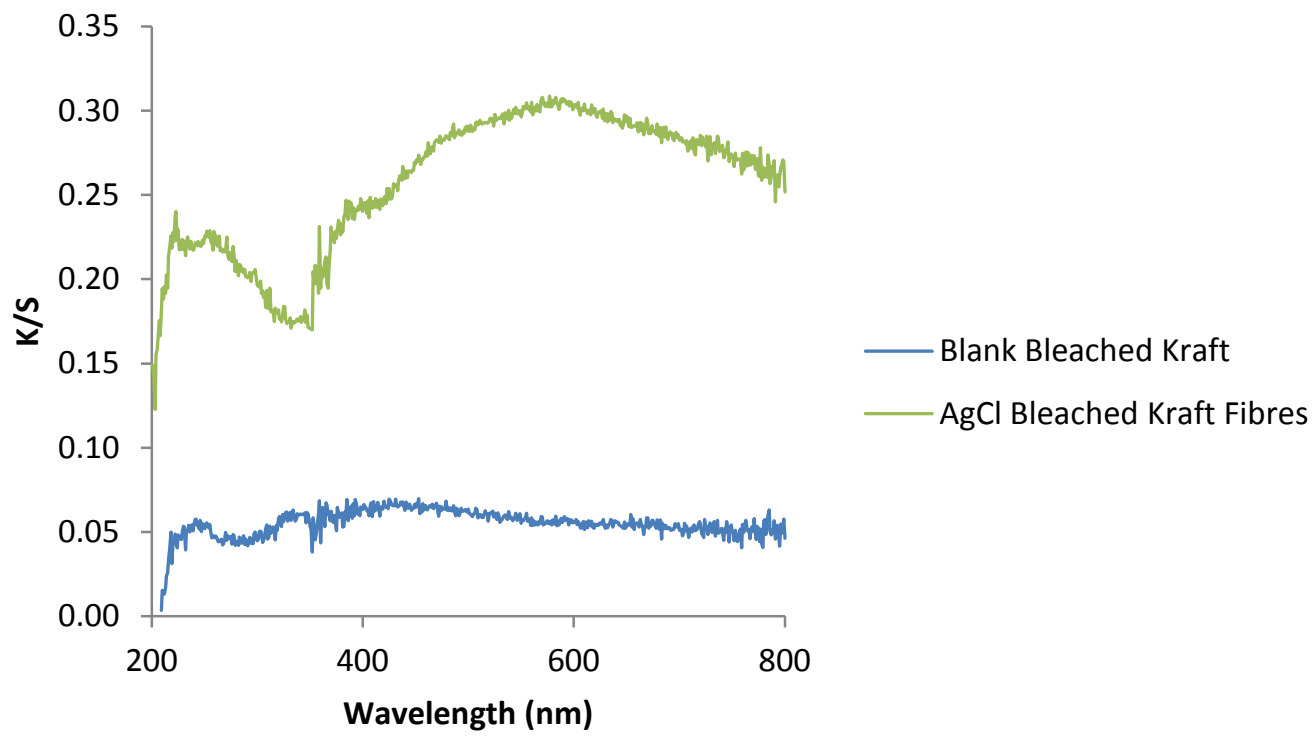

Figure $51 \mathrm{UV} / \mathrm{Vis}$ of $\mathrm{AgCl}$ Bleached kraft Fibres after light exposure 


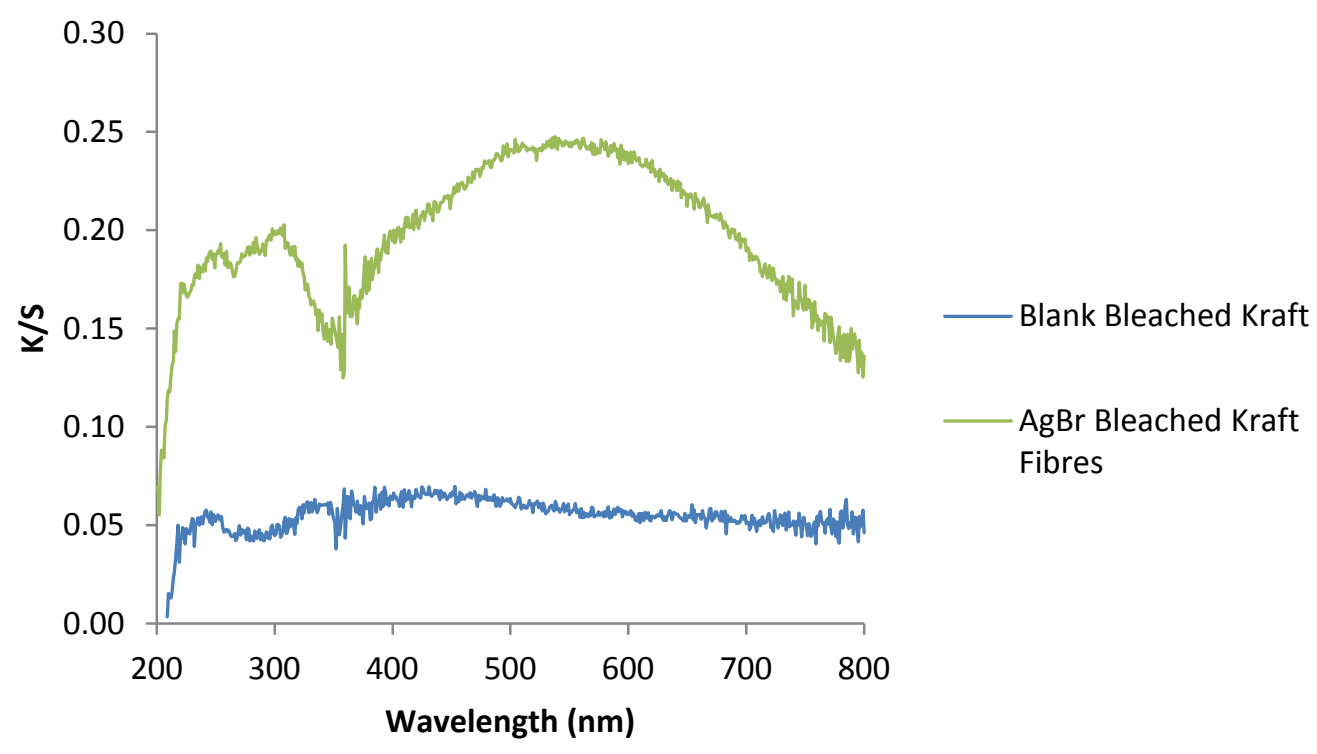

Figure $52 \mathrm{UV} / \mathrm{Vis}$ of $\mathrm{AgBr}$ Bleached kraft Fibres after light exposure

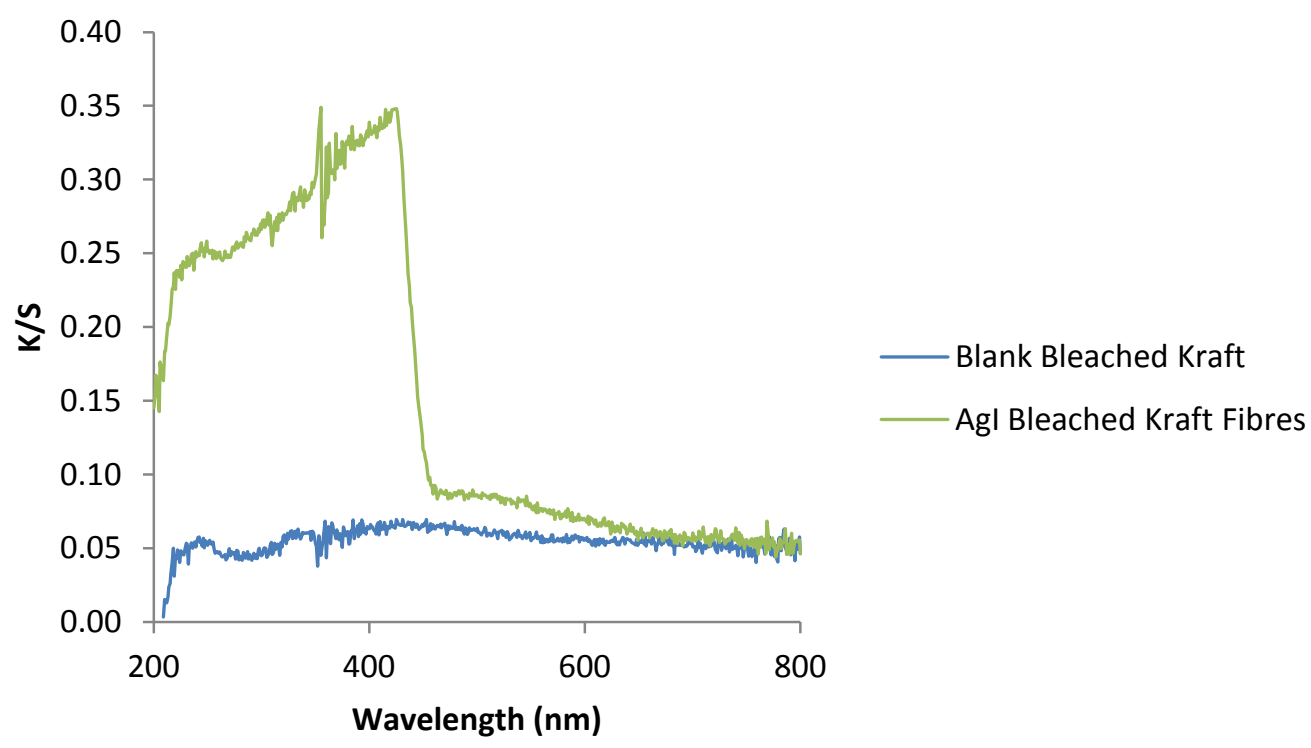

Figure 53 UV/Vis of AgI Bleached kraft Fibres after light exposure

XRD was used to characterise the silver/silver halide kraft nanocomposites. It was a useful tool in determining if silver halides had been formed within the kraft fibre matrix. The high sensitivity of the X'Pert Pro diffractometer again allowed the crystalline silver halides to be identified in conjunction with the kraft fibre matrix diffraction pattern. $\mathrm{AgCl}, \mathrm{AgBr}$ and $\mathrm{AgI}$ 
based nanocomposites were found to have formed their respective silver halides in the kraft fibre samples. Titania $\left(\mathrm{TiO}_{2}\right)$ was found to be present in the kraft fibre samples; $\mathrm{TiO}_{2}$ is a common additive in paper production as it imparts a high whiteness and brightness to the paper sheet. This can be seen in the blank XRD pattern of bleached kraft fibres (Figure 54). Also of note is the large peak at $\sim 23^{\circ} 2$ Theta, which is due to the crystalline cellulose within the kraft fibre matrix. It can also be seen that the light exposed samples of $\mathrm{Ag} / \mathrm{AgCl}$ and $\mathrm{Ag} / \mathrm{AgBr}$ kraft nanocomposites have a small $\mathrm{Ag} 111$ peak coming through in the diffraction profile. Showing that, both crystalline silver and silver halide are present after light exposure. This important as this small amount of silver is responsible for the broad visible absorption developed after light exposure and the photocatalytic properties, through SPR as we have seen with the silver/silver halide nanoparticles. This shows that $\mathrm{Ag} / \mathrm{AgX}$ particles have been formed, after light exposure, in the kraft fibre matrix. AgI kraft nanocomposites showed little indication of any crystalline Ag. This is consistent with the AgI wool nanocomposites due to the lesser sensitivity of $\mathrm{AgI}$ to photolysis as mentioned previously.

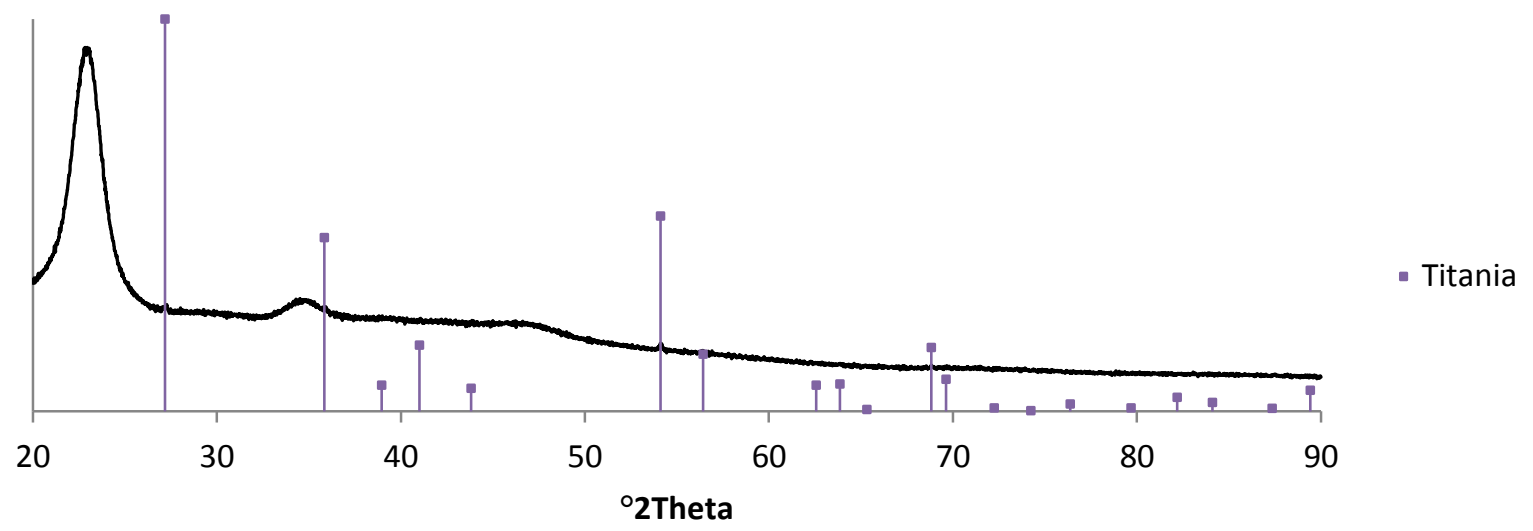

Figure 54 XRD pattern of bleached kraft fibres 


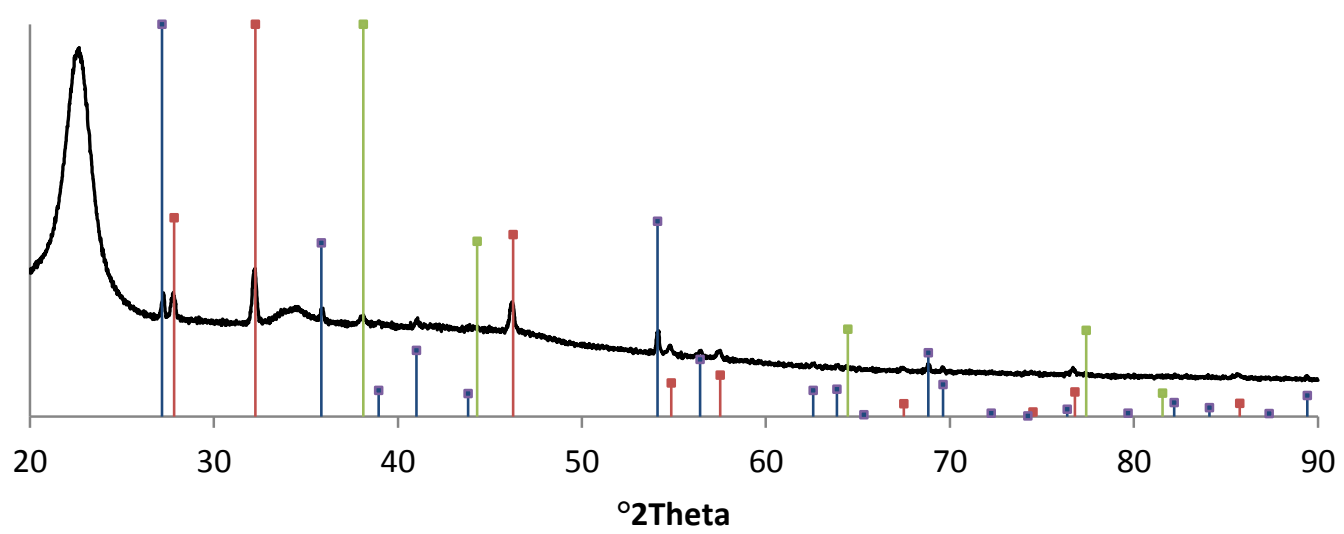

- $\mathrm{Ag}$

- $\mathrm{AgCl}$

- Titania

Figure 55 XRD pattern of $\mathrm{AgCl}$ bleached kraft nanocomposite

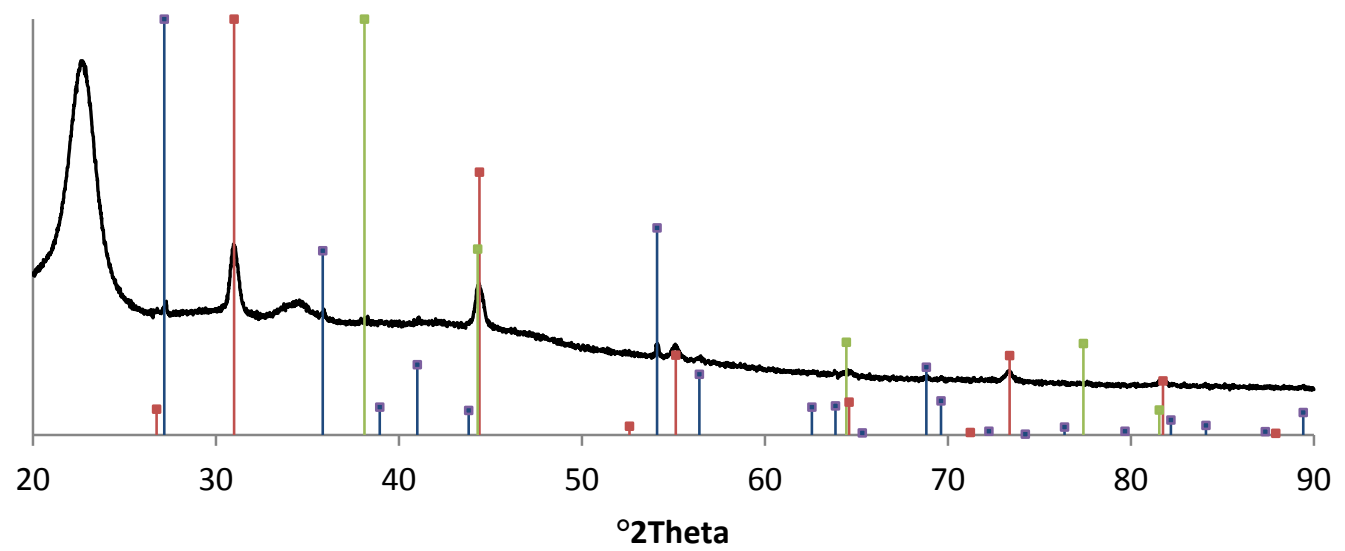

- $\mathrm{AgBr}$

- $\mathrm{Ag}$

- Titania

Figure 56 XRD pattern of $\mathrm{AgBr}$ bleached kraft nanocomposite

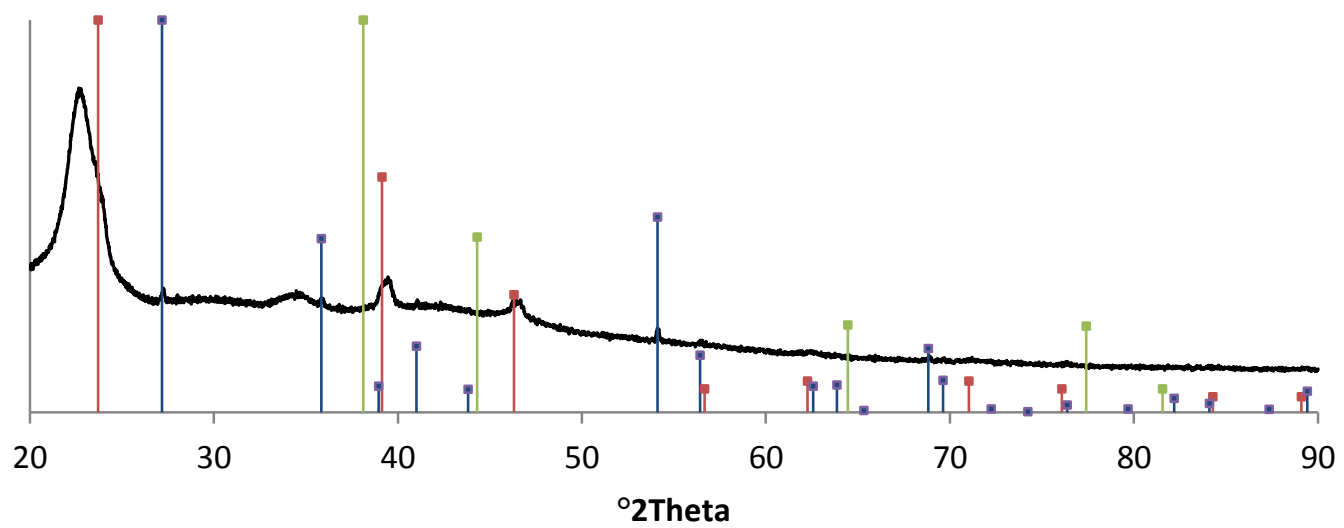

- $\mathrm{Ag}$

- $\mathrm{AgCl}$

- Titania

Figure 57 XRD pattern of AgI bleached kraft nanocomposite 
SEM characterisation was carried out to see the distribution of the silver halide nanoparticles along the kraft fibres. Only bleached kraft fibre nanocomposites were characterised by SEM, however the similar XRD and UV/Vis seen in bleached and unbleached fibres along with the similar fibre structure, would suggest that the unbleached samples are comparable to what is seen in the bleached fibres. SEM confirmed that there were silver halide nanoparticles present in all of the $\mathrm{AgCl}, \mathrm{AgBr}$ and $\mathrm{AgI}$ based kraft fibre nanocomposites. The SEM backscatter images indicated the positions of silver halide nanoparticles as bright areas within the image, as the heavier silver atoms appear brighter in backscatter mode. The backscatter images showed that silver halide nanoparticles were distributed along the fibres in a variety of different sized agglomerates and discrete particles. It is also seen that, similar to the silver/silver halide wool nanocomposites, the particles are mostly beneath the surface of the fibre. However unlike wool fibres, kraft fibres are a lot thinner. So although the majority of nanoparticles are below the surface, there are some on the surface of the fibres, whereas little to no surface particles were seen in the wool nanocomposites. This is seen in the SEI images showing some silver halide nanoparticles protruding from the kraft fibre surface, as well as in cross-sectional analysis discussed further with the EDS analysis of the kraft nanocomposites. The edges of the fibres have higher concentrations of agglomerates, but there is also a reasonable distribution of particles throughout the whole fibre. In general the agglomerates seen in the kraft fibre nanocomposites are smaller than those in the wool fibre nanocomposites, as seen in higher magnification images in the appendix.

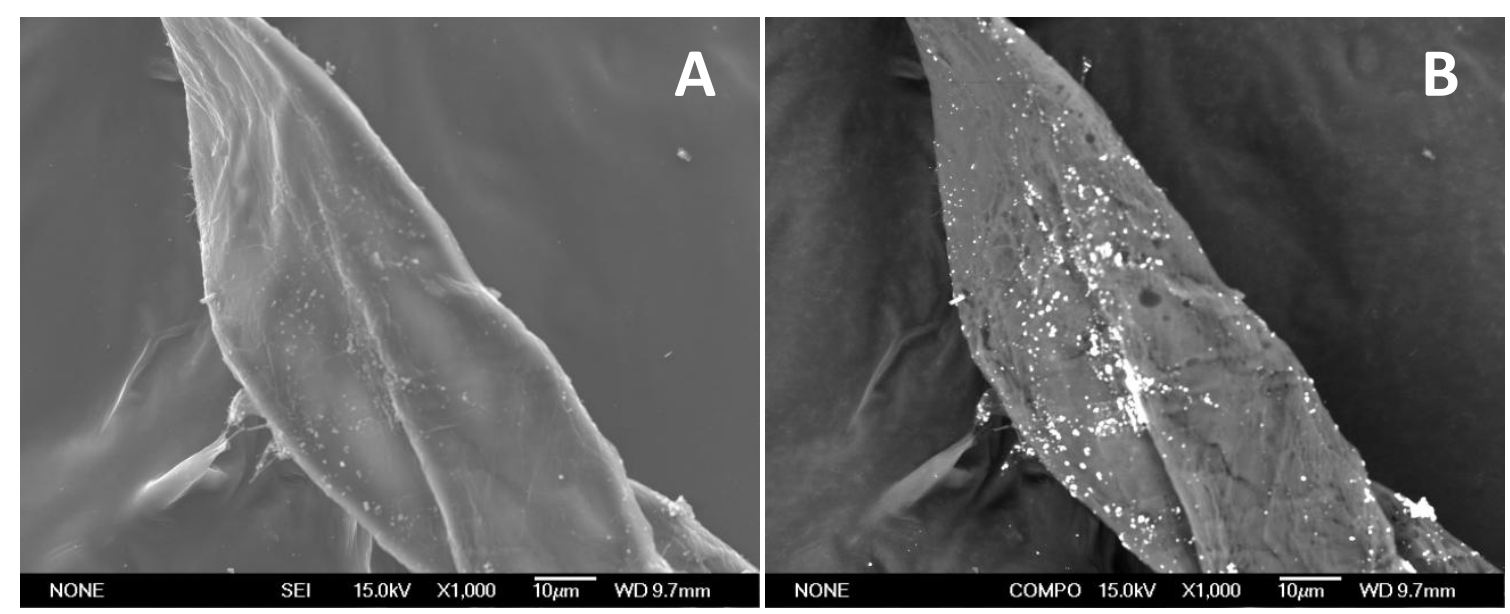

Figure 58 SEM images of $\mathrm{AgCl}$ bleached kraft fibre nanocomposite A) SEl image B) Backscatter image 

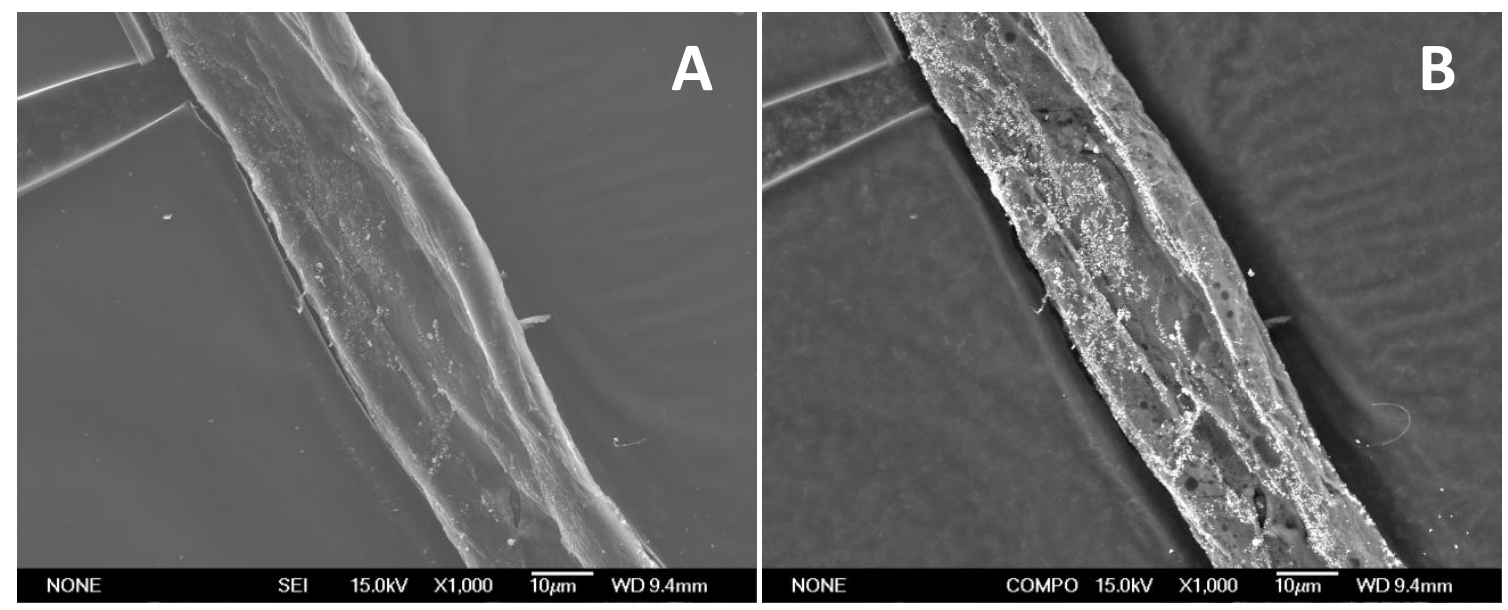

Figure 59 SEM images of AgBr bleached kraft fibre nanocomposite A) SEI image B) Backscatter image
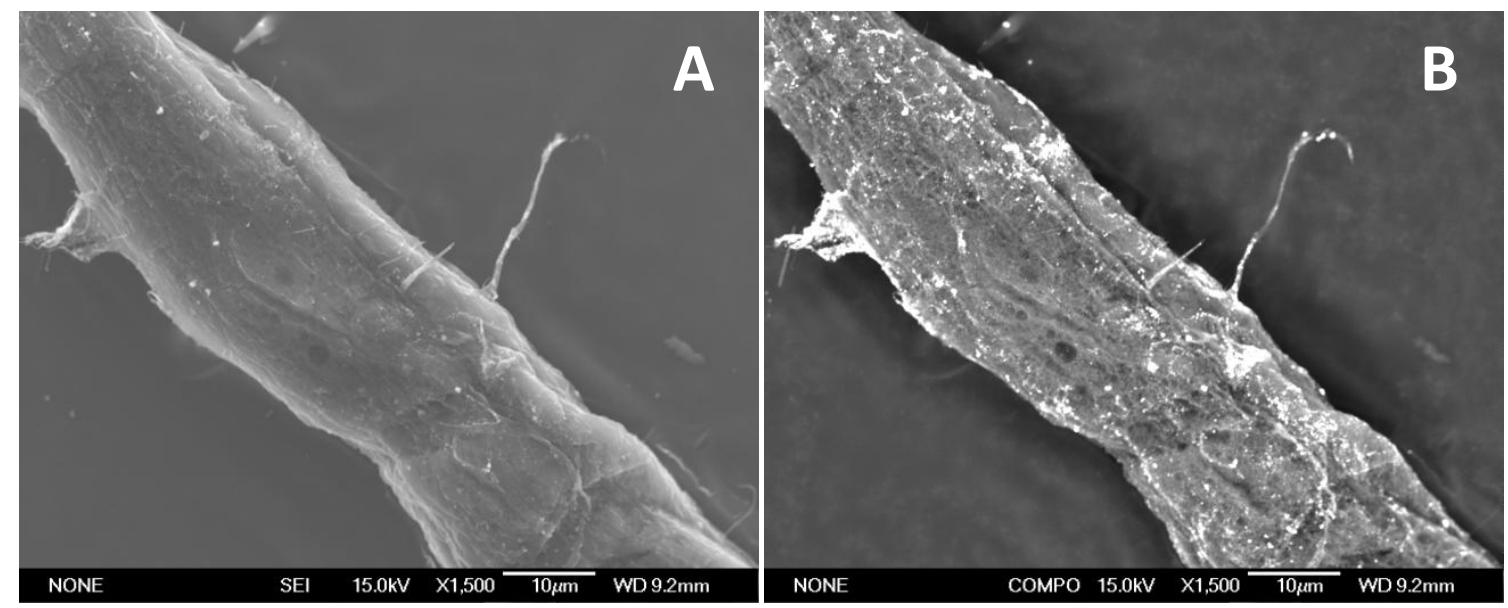

Figure 60 SEM images of AgI bleached kraft nanocomposite A) SEI image B) Backscatter image

The SEM was also used to carry out EDS analysis of the silver/silver halide kraft fibre nanocomposites. This was used to confirm the presence of the silver halide nanoparticles within the kraft fibres, and to view the distribution of nanoparticles. There was good overlap, as can be seen in the EDS maps, for all of Ag with their respective halides. The maps show that areas of high Ag concentration also have high halide concentrations, further confirming that there has in fact been silver halide nanoparticle formation within the kraft fibres during synthesis. These areas of high $\mathrm{Ag}$ and halide concentration are also in accordance with the backscatter SEM. This good correlation indicates that the bright areas in the backscatter images are silver halide nanoparticles. 


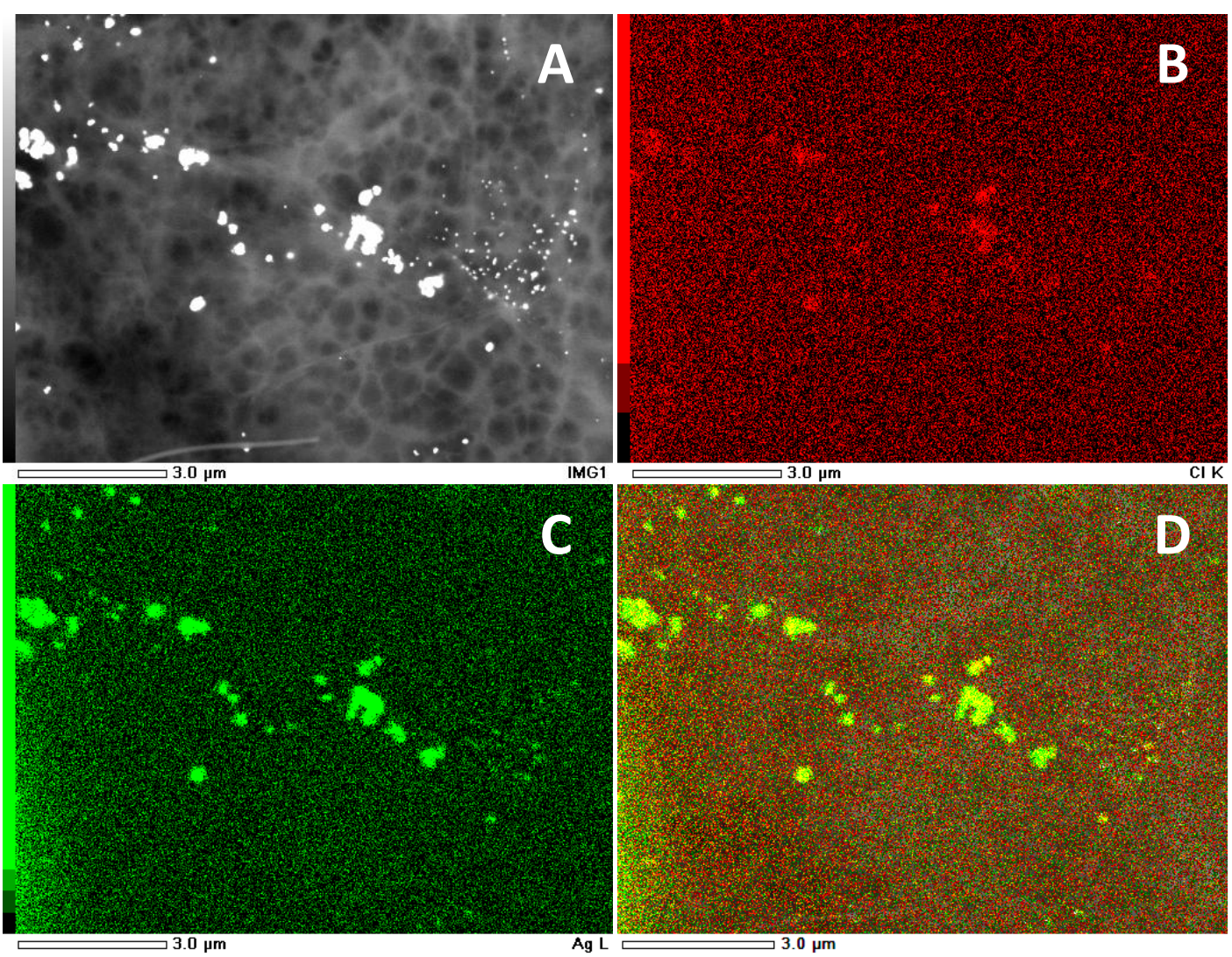

Figure $61 \mathrm{AgCl}$ kraft nanocomposite A) SEM backscatter image B) EDS map of Cl C) EDS map of Ag D) EDS overlay
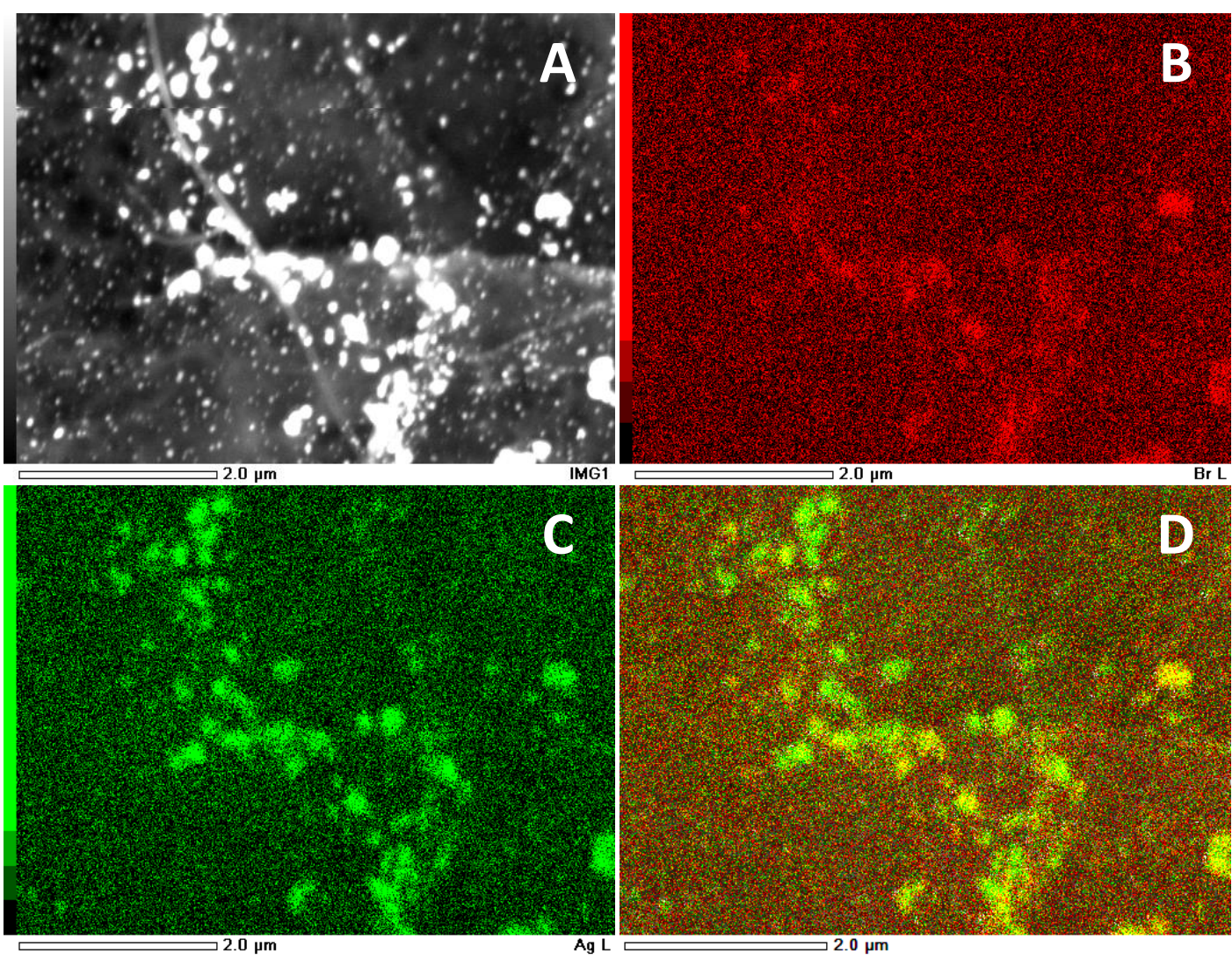

Figure $62 \mathrm{AgBr}$ kraft nanocomposite A) SEM backscatter image B) EDS map of $\mathrm{Br}$ C) EDS map of $\mathrm{Ag}$ D) EDS overlay 


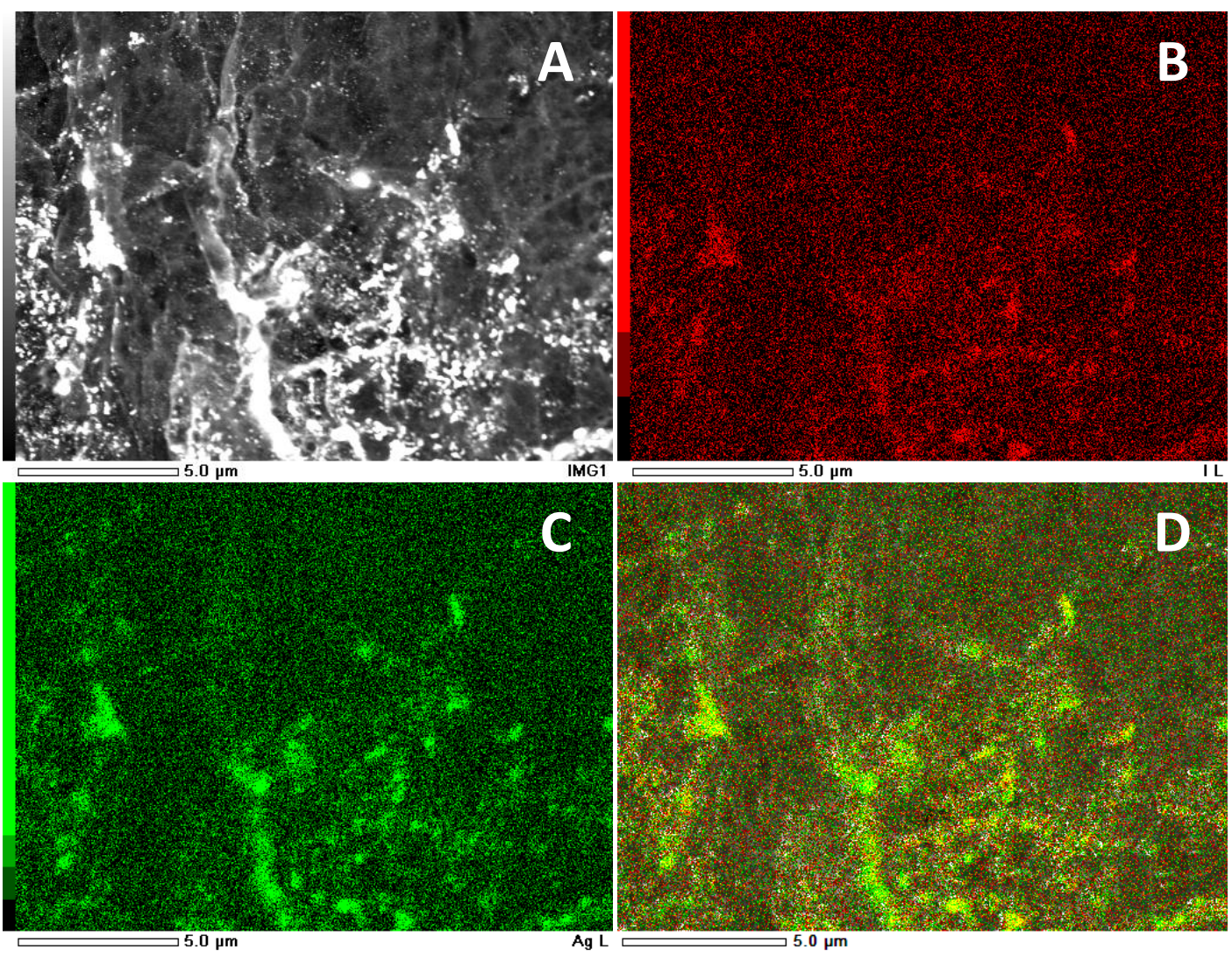

Figure 63 Agl kraft nanocomposite A) SEM backscatter image B) EDS map of I C) EDS map of Ag D) EDS overlay

Cross-sectional SEM of the silver/silver halide kraft fibre nanocomposites was carried out with EDS. This cross-sectional analysis revealed that the centre of the fibres had little or no silver halide nanoparticles. It shows that the nanoparticles form largely just below the surface of the fibres, not in the centre of the fibre. This is similar to the silver halide wool nanocomposites, however as mentioned the thinner nature of the kraft fibre means some nanoparticles that are present close to the centre of the fibres. With these results the mechanism of silver halide formation within the kraft fibres would be the same as the silver halide wool nanocomposites.

This is the mechanism is via precipitation, where growth is controlled by the matrix. As described previously the doped fibres have halide ions distributed throughout. Washing removes surface halide ions leaving only halide ions within the fibre. Upon immersion in the silver nitrate solution the $\mathrm{Ag}^{+}$ions absorbed into the fibre matrix and retained. The halide 
ions then migrate towards the $\mathrm{Ag}^{+}$ions. The silver halide is then formed through precipitation as silver halides are very insoluble in aqueous environments. This is further supported by nanocomposites being made using both bleached and unbleached fibres. This shows that the functional groups of lignin are not required to reduce or stabilise the silver halides to form nanoparticles.

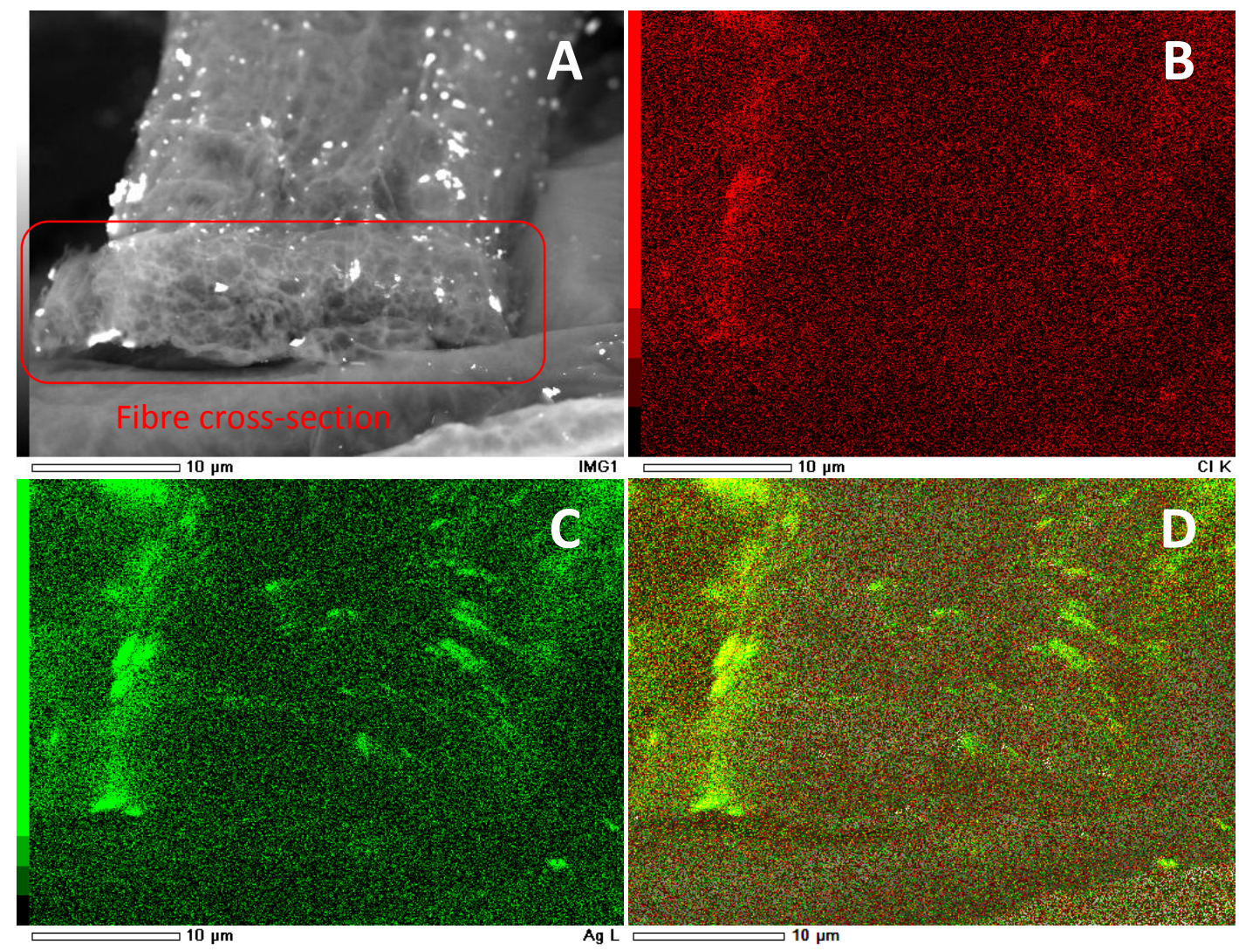

Figure $64 \mathrm{AgCl}$ kraft cross section A) SEM backscatter image B) EDS map of Cl C) EDS map of Ag D) EDS overlay 


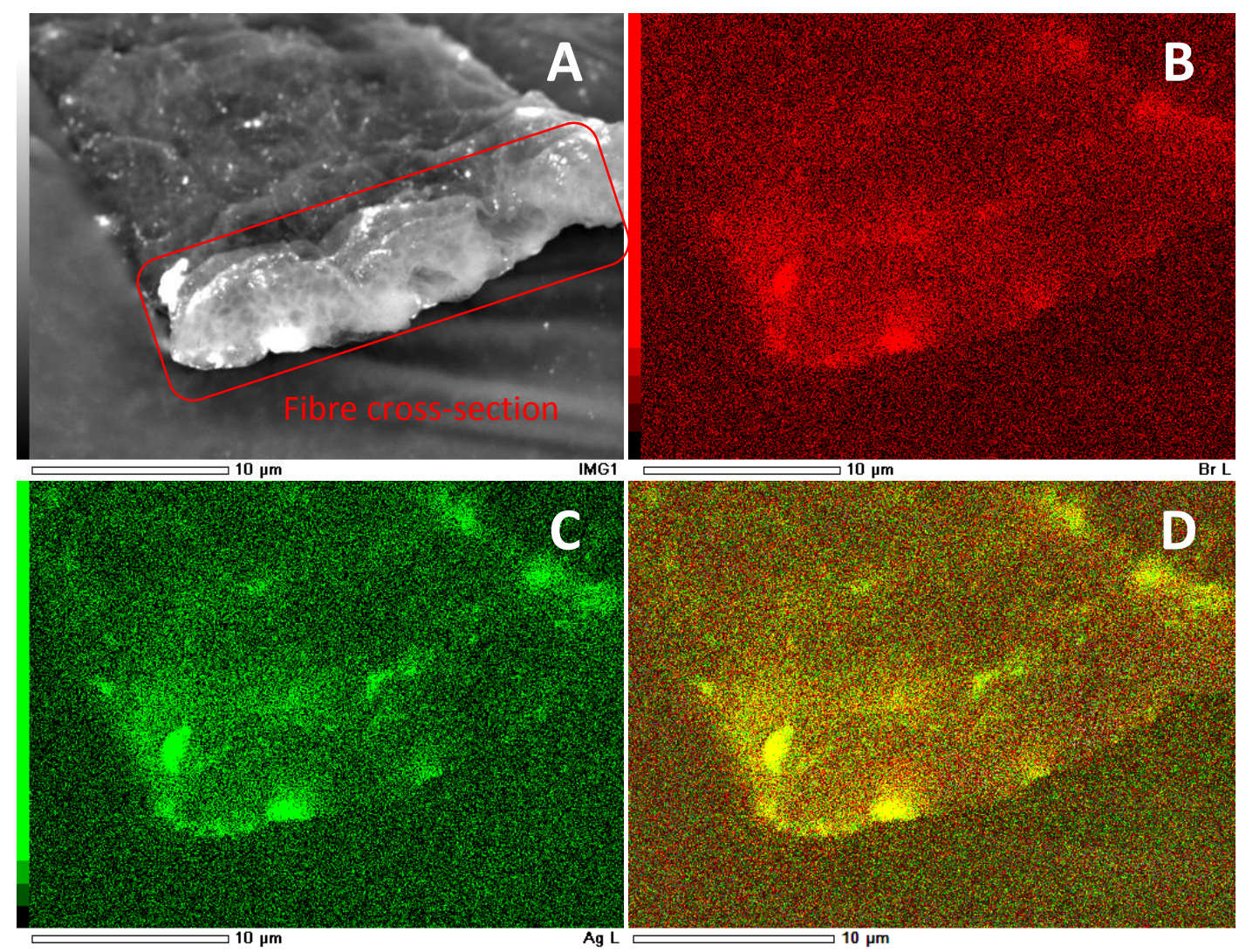

Figure $65 \mathrm{AgBr}$ kraft cross section A) SEM backscatter image B) EDS map of Br C) EDS map of Ag D) EDS overlay
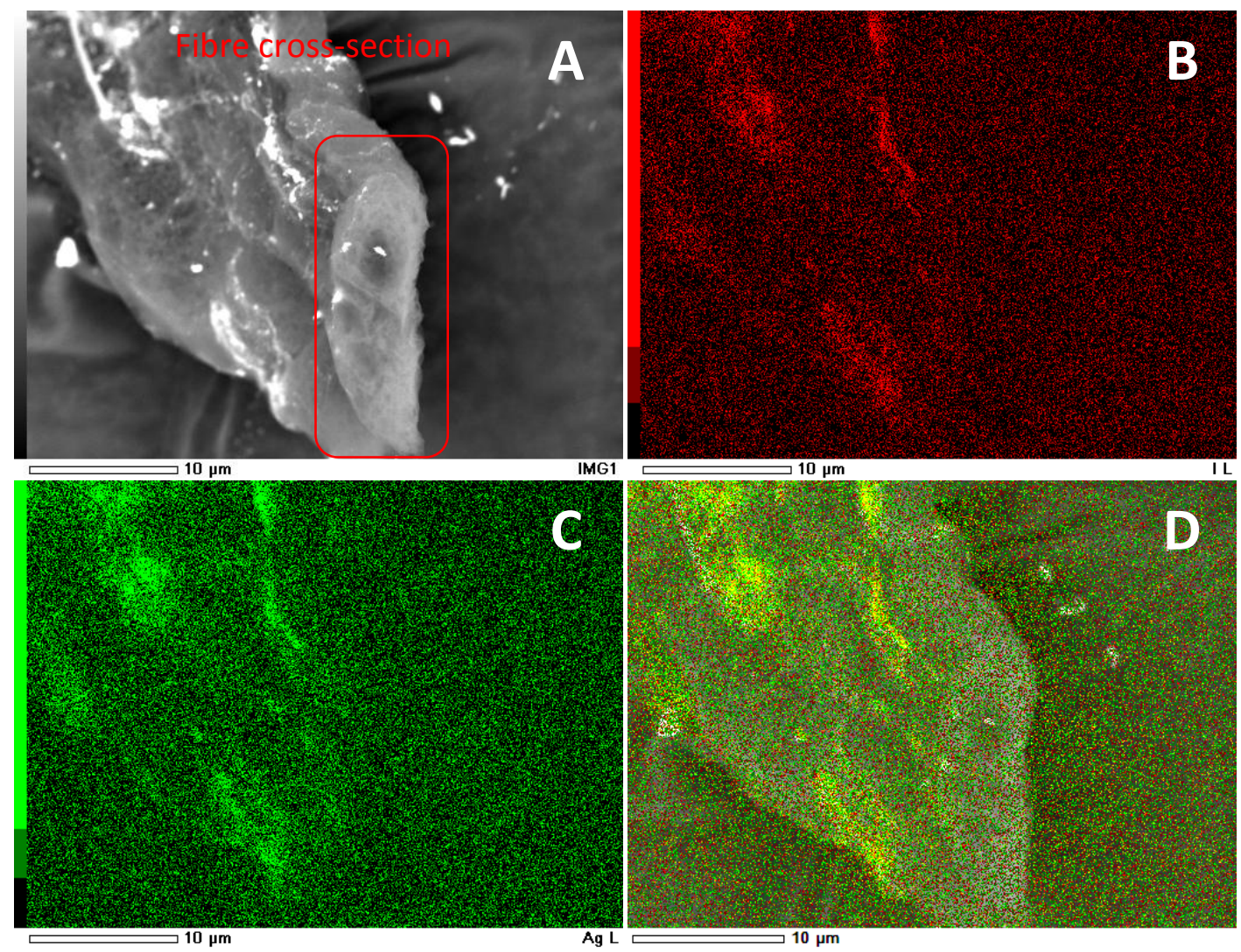

D

Figure 66 Agl kraft cross section A) SEM backscatter image B) EDS map of I C) EDS map of Ag D) EDS overlay 
In summary it has been shown that silver/silver halide kraft nanocomposites of $\mathrm{AgCl}, \mathrm{AgBr}$ and AgI have been successfully synthesised. XRD and EDS analysis have shown that silver halide nanoparticles are formed within the kraft fibre matrix using both bleached and unbleached kraft fibres. SEM revealed that these nanoparticles form within the fibre just below the fibre surface. UV/Vis spectroscopy and XRD have confirmed that upon light exposure the silver halide nanoparticles undergo photochemical reaction, developing visible region absorption and crystalline silver for $\mathrm{AgCl}$ and $\mathrm{AgBr}$ based nanocomposites. These are characteristic of the silver/silver halide nanoparticles used as plasmonic photocatalysts referred and discussed earlier. The silver/silver halide nanocomposites have potential to be plasmonic photocatalysts stabilised on a kraft fibre matrix. The synthesis using both unbleached and bleached kraft, as well as wool fibres, also further indicates that synthesis is through precipitation of silver halide nanoparticles within the matrix.

\subsection{Silver/Silver Halide Nanostructured Calcium Silicate Nanocomposites}

The successful synthesis of silver/silver halide nanocomposites using both wool and paper fibre matrices showed that fibre composition was not a determining factor for synthesis. Furthermore, the use of bleached and unbleached kraft demonstrated that the functional groups of the fibres were not required for synthesis of the silver/silver halide nanocomposites. With this, the opportunity to form many different nanocomposites was realised. The simple mechanism of precipitation and nanoparticle size control through pore size of the matrix opens up various porous materials to be a matrix for silver/silver halide nanocomposites. Nanostructured calcium silicate (NCS) is proprietary material, developed by Prof J. H. Johnston through Victoria University of Wellington, with a large surface area and highly porous structure. ${ }^{71}$ Silver functionalised NCS had been previously synthesised, and had on occasion shown to develop some purple colouration with time. ${ }^{74}$ As the NCS synthesis process involves $\mathrm{HCl}$, the structure contains residual $\mathrm{Cl}^{-}$ions trapped within the porous structure. These ions potentially initiated formation of small amounts of $\mathrm{AgCl}$ and through photolysis caused a colour change upon exposure to light. NCS then became an obvious silver/silver halide nanocomposite possibility to be produced. 
Using the experimental method described in the experimental section, silver/silver halide NCS nanocomposites were synthesised. $\mathrm{AgCl}, \mathrm{AgBr}$, and $\mathrm{AgI} \mathrm{NCS}$ nanocomposites were all formed. $\mathrm{AgCl}$ and $\mathrm{AgBr}$ based NCS nanocomposites also showed the characteristic colour change from the white of the NCS to purple once exposed to light, as seen with the previous nanocomposites. The AgI NCS nanocomposite samples displayed similar characteristics to the AgI wool and Kraft nanocomposites, in that when synthesised they developed a yellow colour, which remained with light exposure. In $\mathrm{AgCl}$ and $\mathrm{AgBr}$ composites this colour is indicative of the formation of the silver/silver halide particle within the nanocomposites. The light induced formation of silver nanoregions on the silver halide nanoparticles is responsible for a broad visible absorption through SPR in the same way seen for previous nanocomposites. ${ }^{32,38,45}$

UV/Vis reflectance spectroscopy was undertaken to characterise the silver/silver halide NCS nanocomposites. The nature of the NCS samples being a powder made it difficult to gain good UV/Vis reflectance from the spectrometer. What can be seen is the increased visible region absorption that is seen in all samples when compared to the blank. Again, $\mathrm{AgCl}$ and $\mathrm{AgBr}$ samples display very similar spectra with broad visible region absorption. (Figure $67 \&$ 68) The AgI NCS nanocomposite does not have a clearly defined absorption, as seen in other AgI nanocomposite samples. There is broad visible region absorption, and at wavelengths $<450 \mathrm{~nm}$ there is increased absorption as indicated with an arrow in figure 69. This area of increased absorption is the equivalent of the very sharp increased absorbance seen in the kraft and wool AgI nanocomposites. This would be expected from the AgI nanoparticles. However it is nowhere near as pronounced as in wool and kraft nanocomposites, due to issues with the NCS in reflectance spectroscopy. 


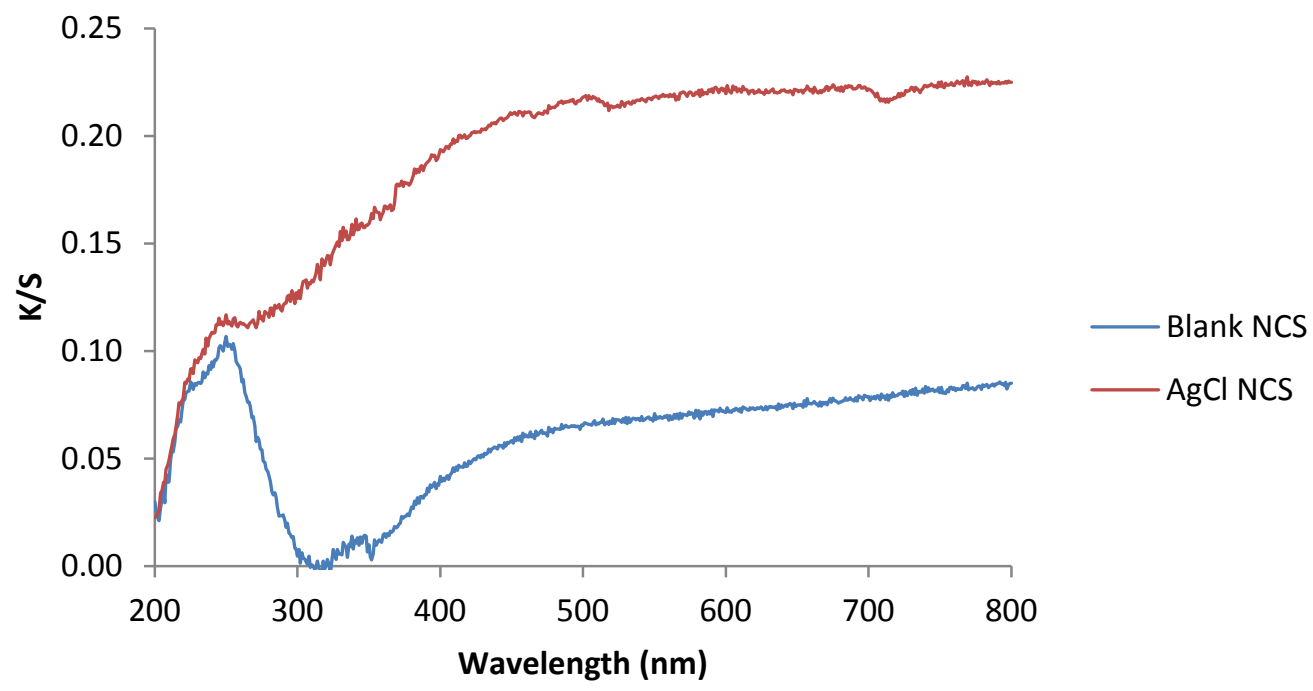

Figure $67 \mathrm{UV} /$ Vis spectra of AgCl NCS nanocomposite after light exposure

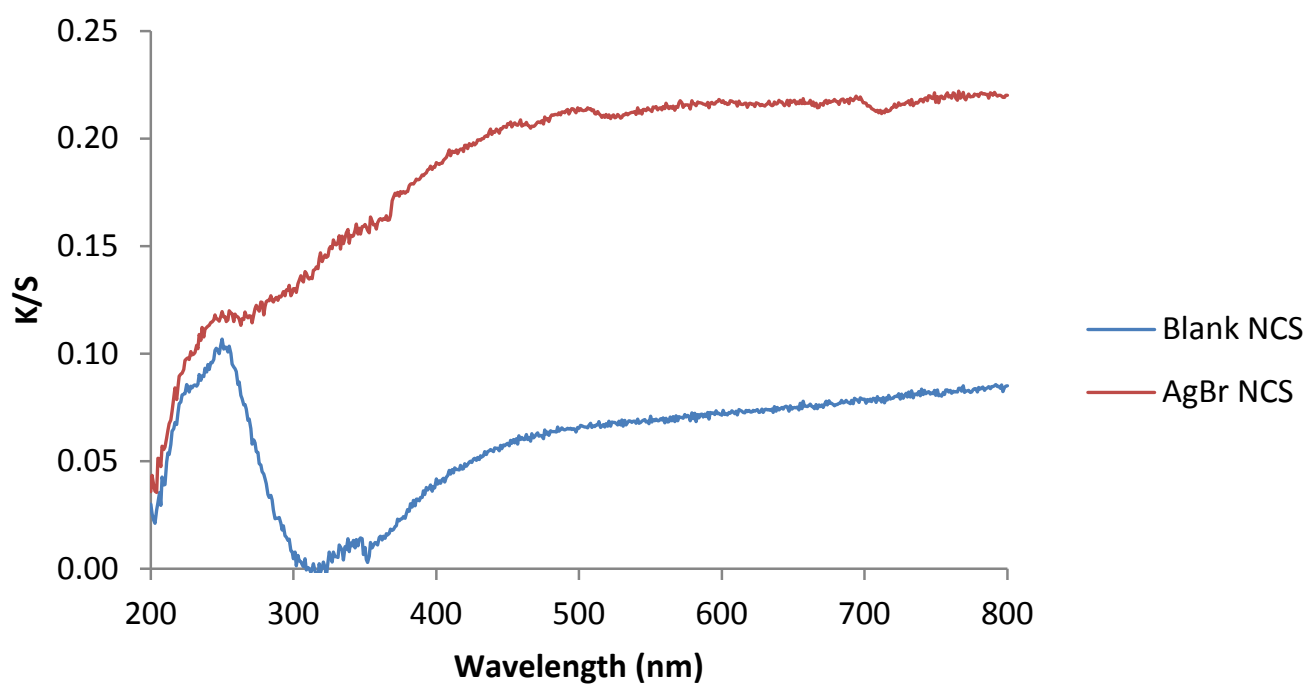

Figure $68 \mathrm{UV} /$ Vis spectra of AgBr NCS nanocomposite after light exposure 


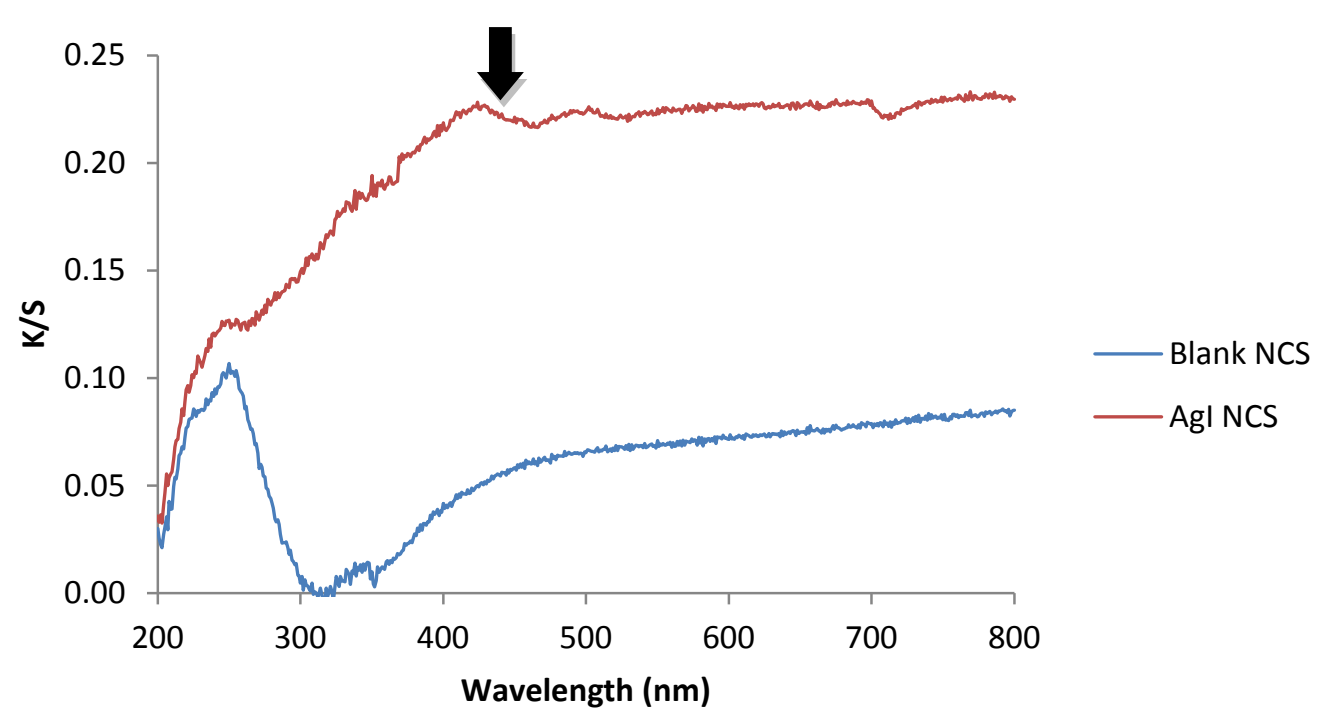

Figure 69 UV/Vis spectra of AgI NCS nanocomposite after light exposure

XRD was used to characterise the silver/silver halide NCS nanocomposites. As with the other nanocomposite samples, XRD determed if silver halide nanoparticles had formed within the NCS matrix during synthesis. The NCS matrix itself does however have some semi crystalline characteristics, and has a diffraction pattern of its own due to the $2 \mathrm{D}$ order of the platelets in the material. ${ }^{72,73}$ (Figure 70) Despite having this reasonably complex background pattern for the NCS matrix XRD showed the presence of crystalline silver halides. All the silver halides explored in this project - $\mathrm{AgCl}, \mathrm{AgBr}$ and $\mathrm{AgI} \mathrm{NCS}$ nanocomposites - can be seen to have formed. After light exposure and the colour development, the $\mathrm{AgCl}$ and $\mathrm{AgBr}$ nanocomposites showed a small crystalline Ag 111 diffraction peak. This indicates that, like the wool and Kraft fibre nanocomposites, both crystalline silver and silver halide are present after light exposure. (Figure 71\& 72) These small amounts of silver are responsible for the broad visible absorption and colour change that develops after light exposure. ${ }^{42,45,48} \mathrm{AgI}$ once again showed little indication of formation of Ag. There were no Ag 111 characteristics seen in the XRD pattern of the AgI NCS nanocomposite. (Figure 73) 


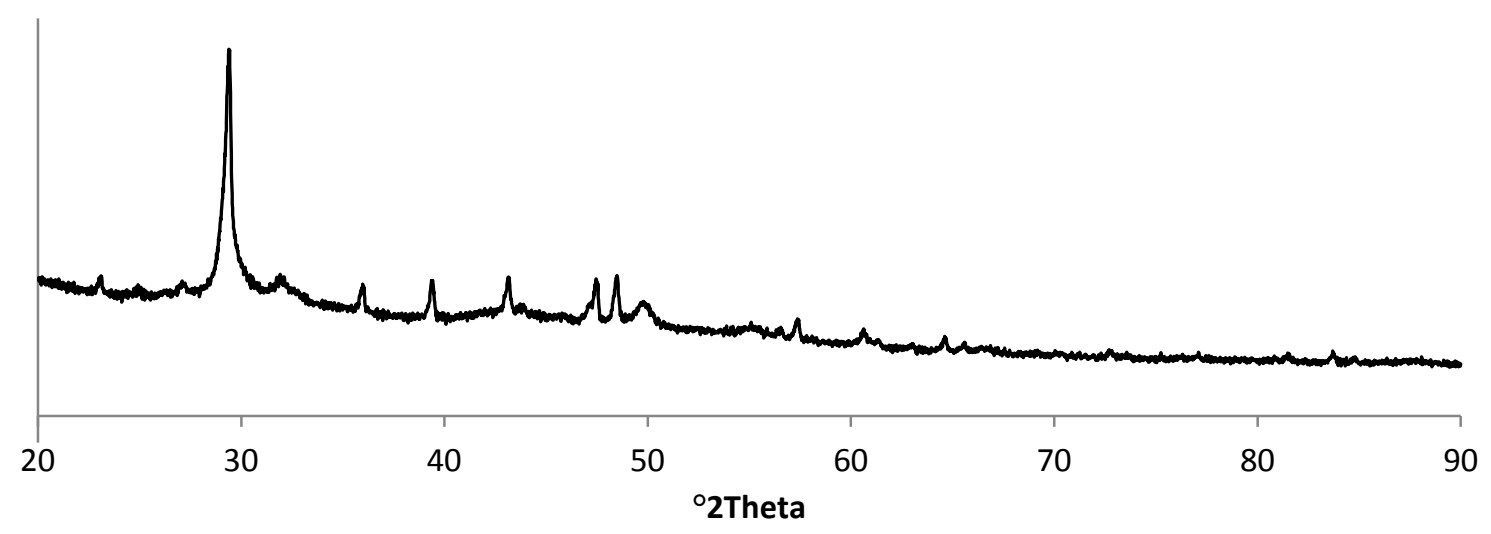

Figure 70 XRD pattern of Blank NCS

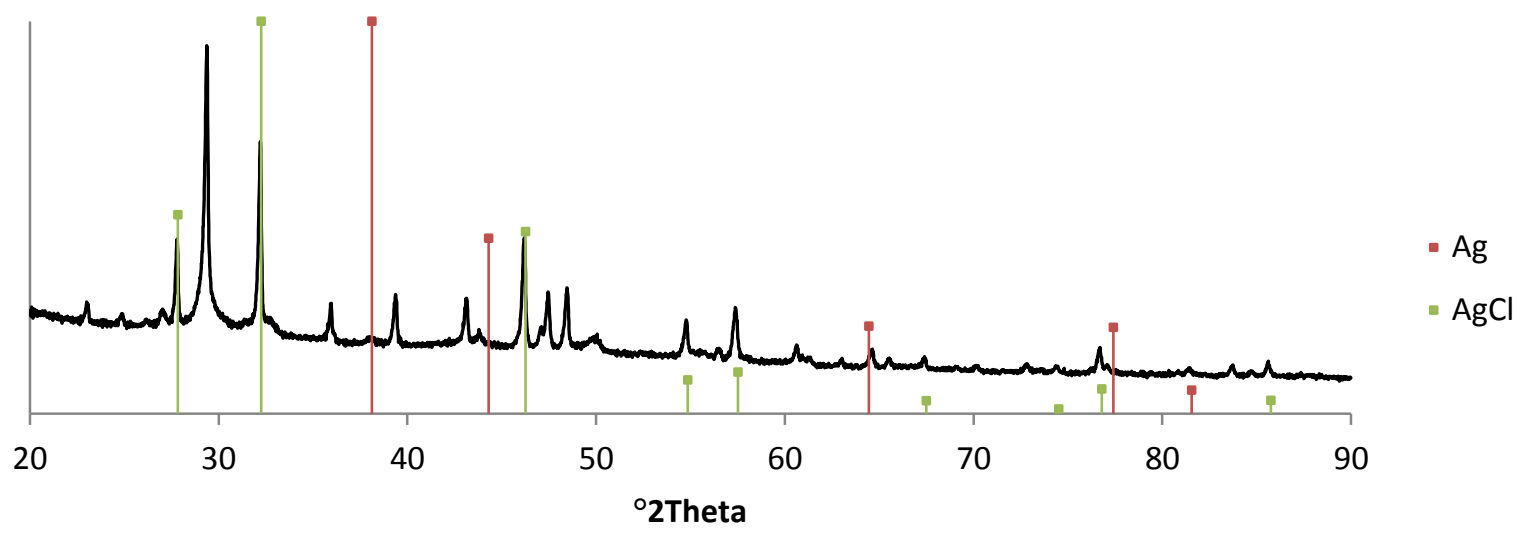

Figure 71 XRD pattern of AgCl NCS nanocomposite

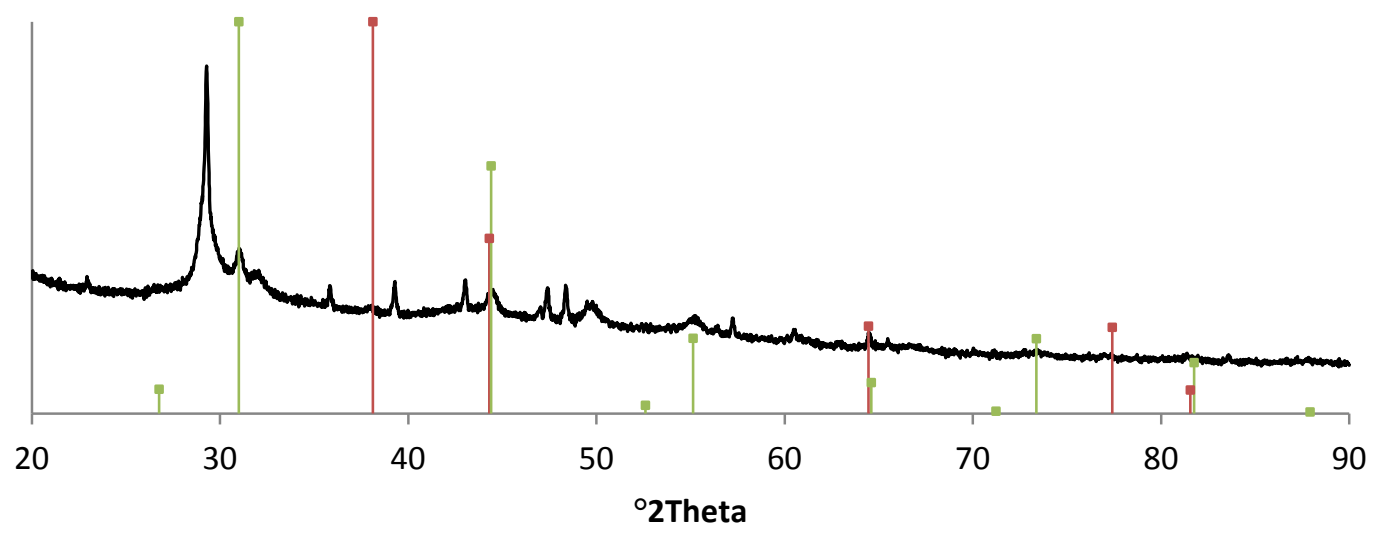

$$
\text { - } \mathrm{Ag}
$$$$
\text { - } \mathrm{AgBr}
$$

Figure 72 XRD pattern of AgBr NCS nanocomposite 


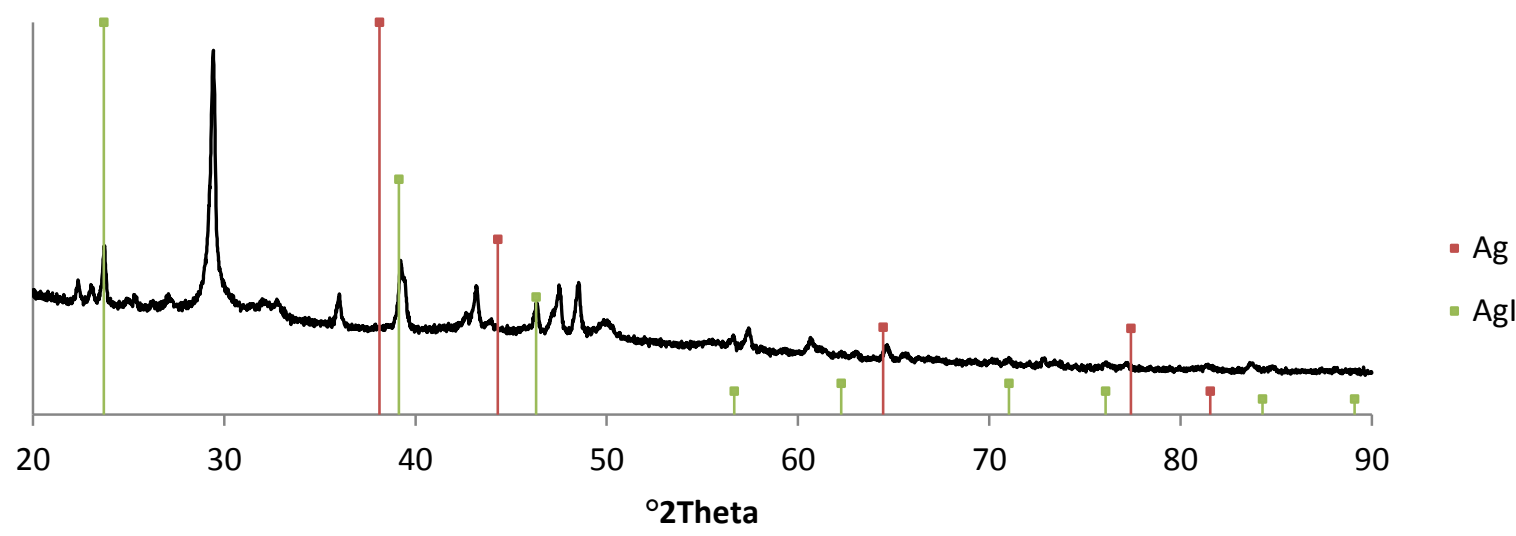

Figure 73 XRD pattern of AgI NCS nanocomposite

SEM analysis of the silver/silver halide NCS nanocomposites was carried to observe the morphology of the composites and to confirm the formation of silver halide nanoparticles within the matrix. The SEI images show that the silver/silver halide NCS nanocomposite maintains its gypsum desert rose like microstructure, and it is not destroyed with the formation of the silver halide nanoparticles. The silver halide nanoparticles are indicated as bright areas in the backscatter SEM images. The silver halide nanoparticles in the NCS appear to be more disperse than in the cases of the fibre nanocomposite matrices seen previously. There does not seem to be any sort of pattern of formation within the NCS matrix, with agglomerates and discrete particles of different sizes forming at random throughout the structure. It does appear that the silver halide nanoparticles have formed within the gypsum desert rose structure of the NCS, and not just on the surface. The differences in the size and shape of the particles may well be due to the size and shape of the open pore structure of the NCS controlling growth of the silver halide particles poorly. The more constrictive porous structures of wool and kraft fibres provide a more restrictions on silver halide growth within the matrix. This means the more porous and variable structure of the NCS shows less size control and less uniform distribution. 

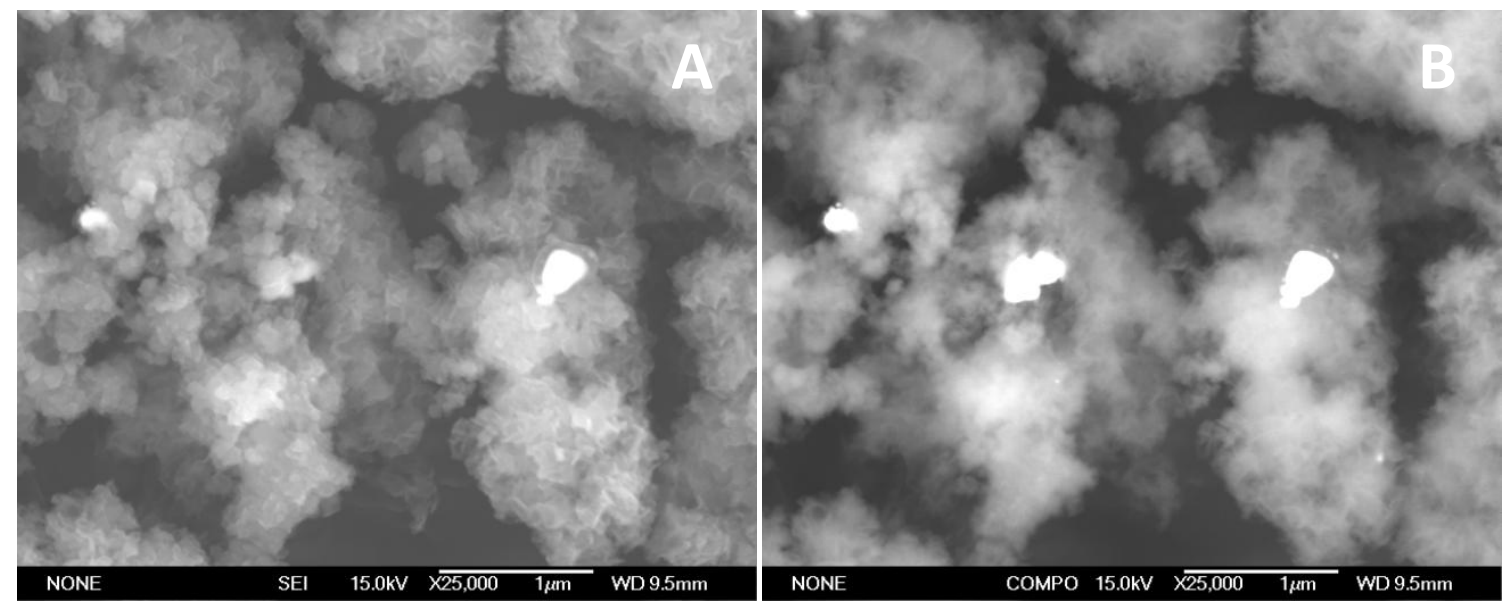

Figure 74 SEM images of AgCl NCS nanocomposite A) SEl image B) Backscatter image

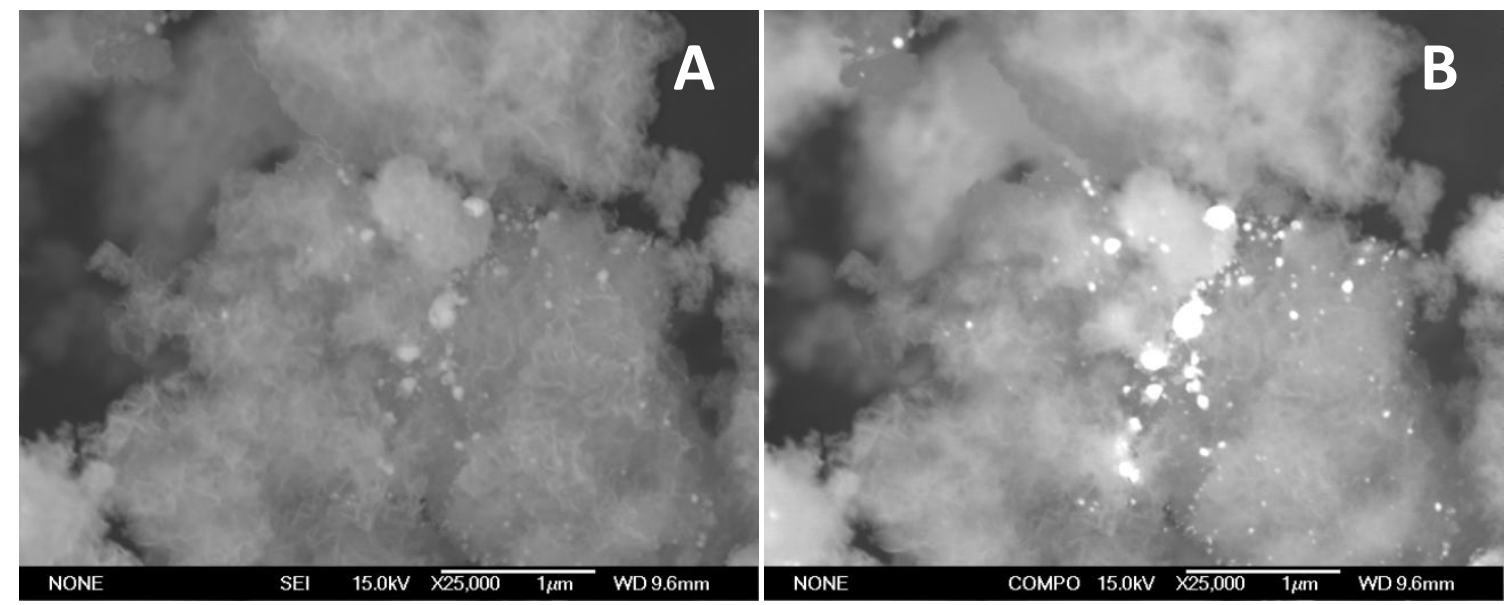

Figure 75 SEM images of AgBr NCS nanocomposite A) SEl image B) Backscatter image
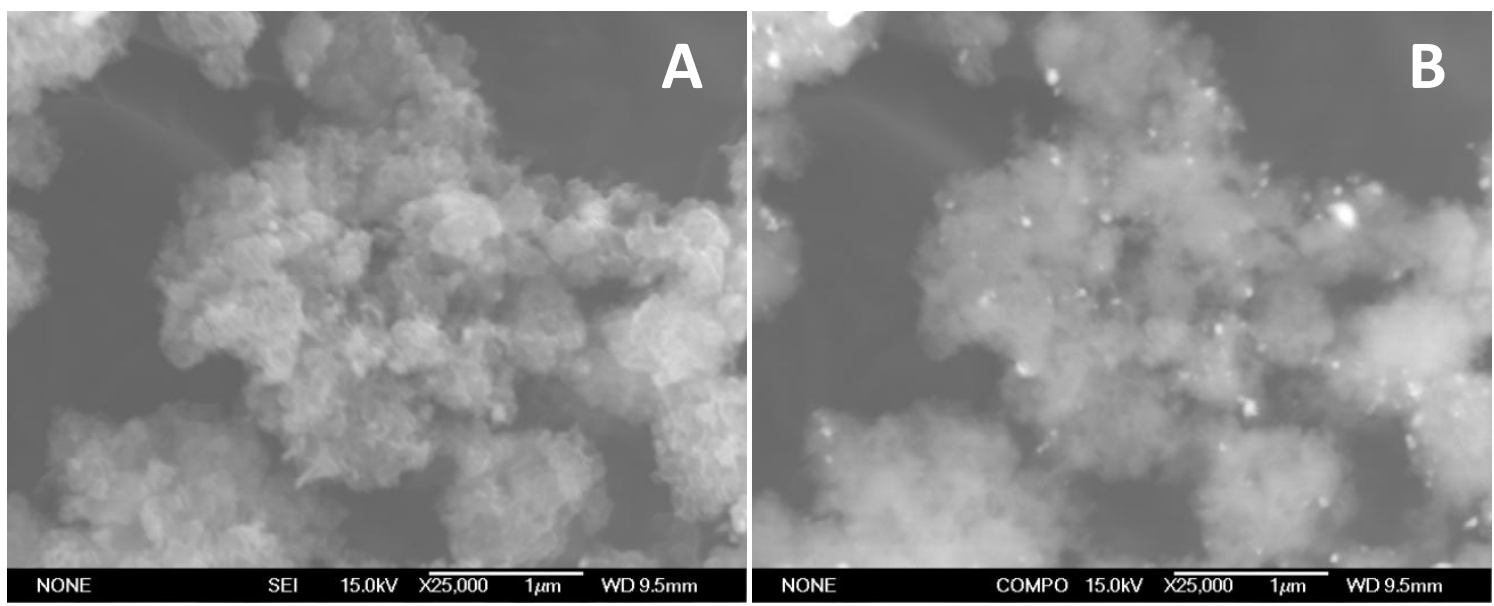

Figure 76 SEM images of AgI NCS nanocomposite A) SEl image B) Backscatter image 
EDS analysis of the silver/silver halide NCS nanocomposites was also carried out using the SEM. This further confirmed the formation of silver halide nanoparticles, and showed that the backscatter images gave a good representation of where silver halide nanoparticles had formed within the NCS. $\mathrm{AgCl}$ and $\mathrm{AgBr}$ NCS nanocomposites show a very good overlap of the $\mathrm{Cl}$ and $\mathrm{Br}$ EDS maps with the corresponding Ag maps showing that the particles are in fact $\mathrm{AgCl}$ and $\mathrm{AgBr}$. (Figure $77 \& 78$ ) The EDS map of the AgI NCS nanocomposite does not show an overlap as strong as the $\mathrm{AgCl}$ and $\mathrm{AgBr}$ nanocomposites. (Figure 79) Areas of higher iodine concentration are difficult to discern from the EDS map. The iodine EDS map shows that iodine is present throughout the NCS matrix. However from the XRD it can be clearly seen that only AgI is present within the sample, and so it is reasonable to assume that the areas of high silver concentration are in fact AgI particles. As the NCS is powder there was no cross sectional analysis to be undertaken as with the fibre samples. The EDS images further demonstrate the poor control of silver halide nanoparticle size and shape control, and so very large and small nanoparticles can be seen.
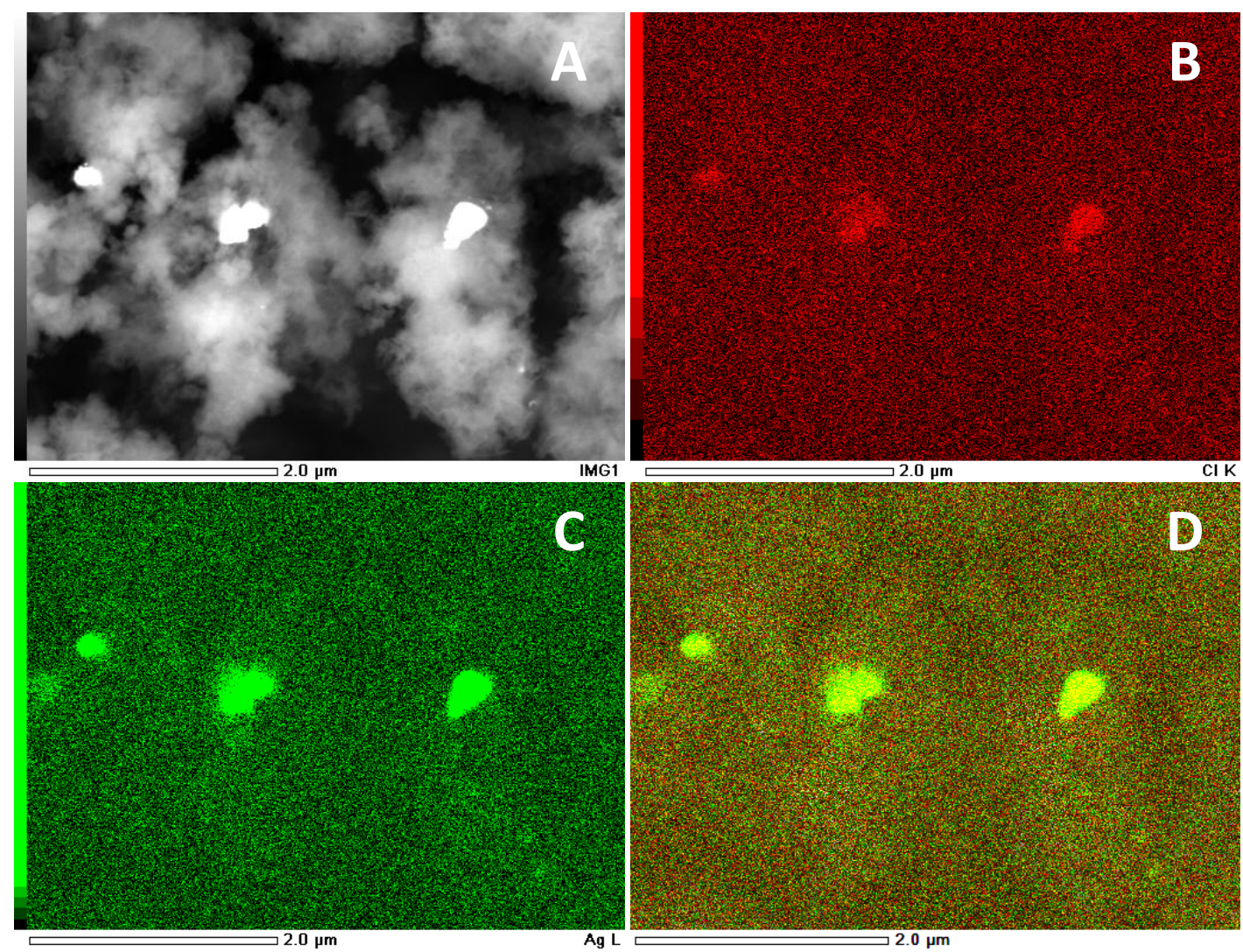

Figure $77 \mathrm{AgCl}$ NCS nanocomposite A) SEM backscatter image B) EDS map of Cl C) EDS map of Ag D) EDS overlay 


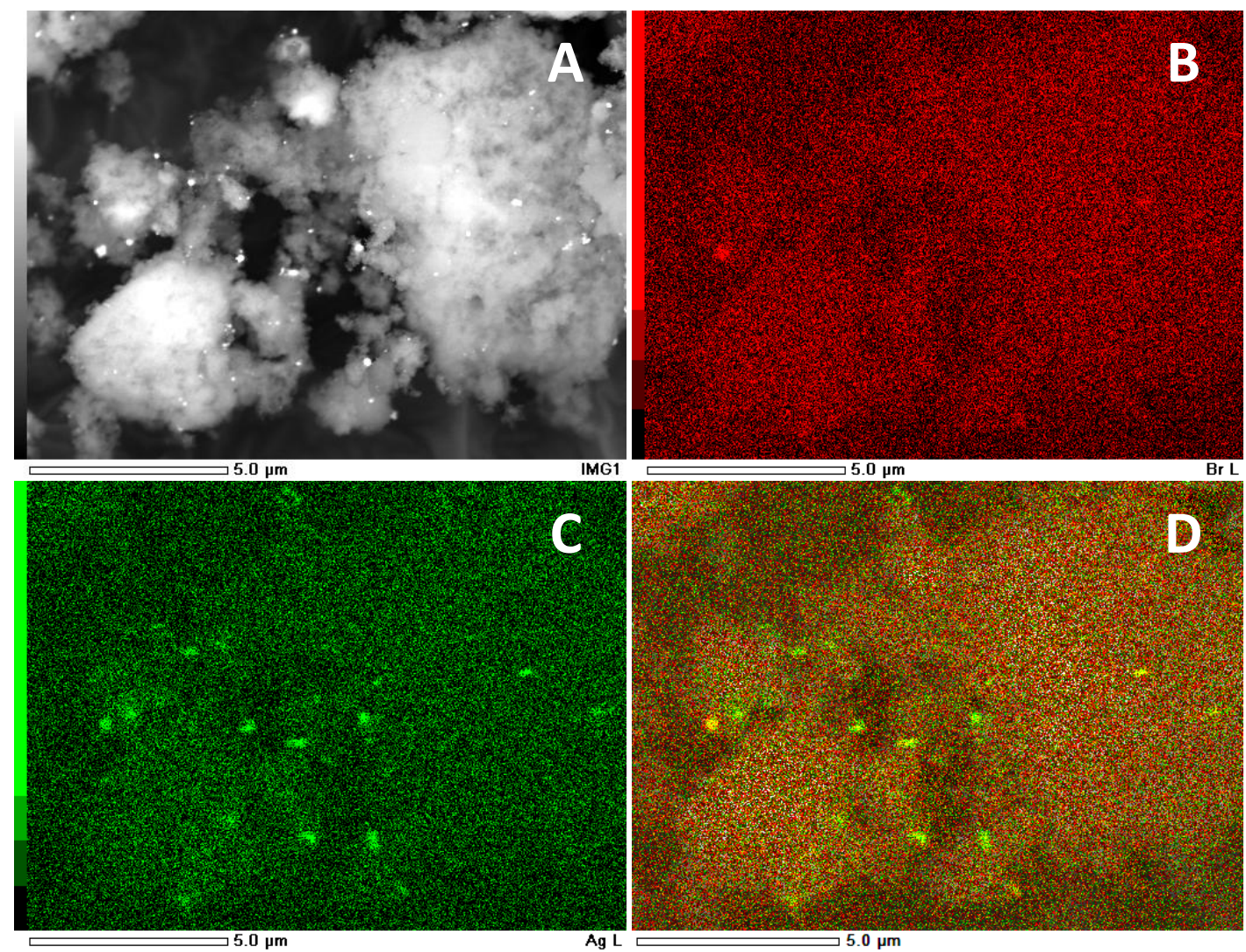

Figure $78 \mathrm{AgBr}$ NCS nanocomposite A) SEM backscatter image B) EDS map of $\mathrm{Br}$ C) EDS map of Ag D) EDS overlay
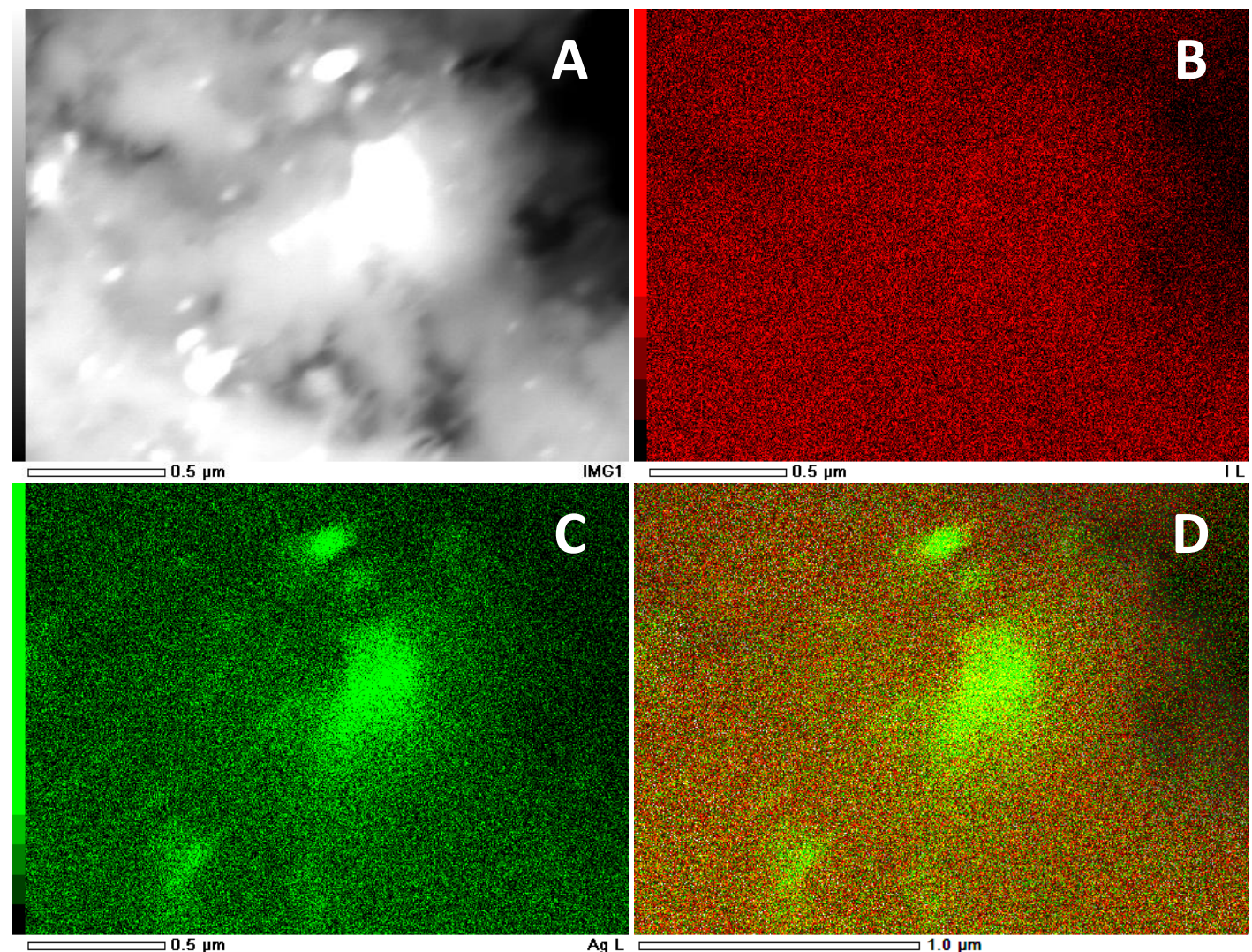

Figure 79 AgI NCS nanocomposite A) SEM backscatter image B) EDS map of I C) EDS map of Ag D) EDS overlay 
From the characterisation of the silver/silver halide NCS nanocomposite samples it can be seen that the synthesis method successfully incorporates silver/silver halide nanoparticles within the NCS matrix. This synthesis is also a relatively simple aqueous method, similar to the synthesis of silver/silver halide wool and kraft nanocomposites. The colour change observed from white to purple after exposure to light for the $\mathrm{AgCl}$ and $\mathrm{AgBr} \mathrm{NCS}$ nanocomposites shows that there is a broad visible region absorption that develops. XRD patterns show the presence of small amounts of crystalline silver within the samples after light exposure. This causes the visible region absorption through the SPR displayed by the silver nanoregions on the silver halide nanoparticles, this is characteristic of the silver/silver halide nanoparticles. ${ }^{42,45,48,49}$ SEM images show that during synthesis the gypsum desert rose structure of the NCS is maintained, allowing the nanocomposites to still have a high porosity and surface area.

The EDS and backscatter images further confirm that there are silver halide nanoparticles within the NCS matrix. The SEM and EDS images also show that the NCS matrix poorly controls size, shape and distribution of the silver/silver halide nanoparticles when compared with the wool and kraft fibre nanocomposites. This characterisation indicates that silver/silver halide NCS nanocomposites have been produced, and with silver/silver halide particles being known photocatalysts these properties could be imparted on to the NCS nanocomposite samples.

\subsection{Evaluation of Photocatalytic Activity}

We have seen that silver/silver halide nanocomposites were successfully synthesised by the aforementioned methods. As previously discussed these silver/silver halide nanoparticles are plasmonic photocatalysts. The common test for determining photoactivity of these particles is through the breakdown of organic dyes. ${ }^{32,35,39,44,45}$ Having synthesised these silver/silver halide nanocomposites it was important to establish if the silver/silver halide nanoparticles maintained their photocatalytic properties when incorporated into a matrix. A preliminary 
evaluation of photocatalytic activity was carried out using the photocatalytic activity tests that have been discussed in the experimental methods. This was with the selected dye, methylene blue as it is commonly used in such experiments. ${ }^{32,40,45}$

The mechanism of dye degradation is through radical formation as discussed in the introduction. With light irradiation the $\mathrm{Ag} / \mathrm{AgCl}$ nanoparticles absorb incident photons, visible light absorption is assisted through SPR, and higher energy UV is absorbed in the typical semiconductor band gap of $\mathrm{AgCl}$ creating an electron-hole pair. The partial polarisation that occurs within the $\mathrm{Ag} / \mathrm{AgCl}$ nanoparticle assists in charge separation, separating the electron-hole pair into the metallic and semiconductor regions of the particle. Then both the electron and hole are used in radical formation. The silver nanodomains and the silver chloride nanoparticle both form radicals through two different mechanisms. The electrons that accumulate on the $\mathrm{Ag}^{0}$ region form super oxide and other radical oxygen species that degrade the organic dye. In a similar manner the silver chloride region also degrades the dye through radical decomposition. The holes that separate into the $\mathrm{AgCl}$ region can themselves oxidise the dye molecules, or alternatively form $\mathrm{Cl}^{0}$ by oxidising $\mathrm{Cl}^{-} . \mathrm{Cl}^{0}$ is a radical species that oxidises organic compounds when in contact with them, reducing $\mathrm{Cl}^{0}$ to $\mathrm{Cl}^{-44,45,79}$ The decomposition of MB by this mechanism allows photocatalytic activity to be measured by dye concentration over time when irradiated.

Due to time constraints, only the silver/silver chloride nanoparticles and nanocomposites photocatalytic activity was evaluated. The samples were irradiated in a Xe arc lamp with MB dye and the absorbance of the MB monitored over time to gauge degradation $(550-700 \mathrm{~nm}) .{ }^{32,}{ }^{40}$ First of all the stability of the MB when in the unfiltered $75 \mathrm{~W} \mathrm{Xe}$ arc lamp had to be established. To rule out direct UV degradation of the dye as being the major factor to lowering absorbance values. It was found that there was slight degradation over a period of hours 24 hours. However, the MB solution was still visibly blue and displayed the MB UV/Vis absorption spectrum. The photoactivity of bulk $\mathrm{AgCl}$ powder also had to be explored, in order to see if $\mathrm{Ag} / \mathrm{AgCl}$ nanoparticles were more efficient photocatalysts. Bulk $\mathrm{AgCl}$ powder showed to increase the breakdown of the $\mathrm{MB}$ in solution (Figure 80), however it was not as efficient as the $\mathrm{Ag} / \mathrm{AgCl}$ nanoparticles. The bulk $\mathrm{AgCl}$ powder also began to show an absorbance at $420 \mathrm{~nm}$, indicating that there had been conversion to Ag nanoparticles 
during the irradiation period for the dye breakdown. This would present limitations in recyclability, as the $\mathrm{AgCl}$ would only be a useful photocatalyst for a small number of cycles as it is converted to $\mathrm{Ag}^{0}$. The $\mathrm{Ag} / \mathrm{AgCl}$ nanoparticles as expected show photocatalytic activity, fully decomposing the MB dye in two hours. (Figure 81) This rate is far faster than the $\mathrm{Xe}$ arc lamp alone or with $\mathrm{AgCl}$. There was also no sign of $\mathrm{Ag}$ nanoparticle formation during the period of irradiation, as would be expected with the self-sensitisation of the nanoparticles.

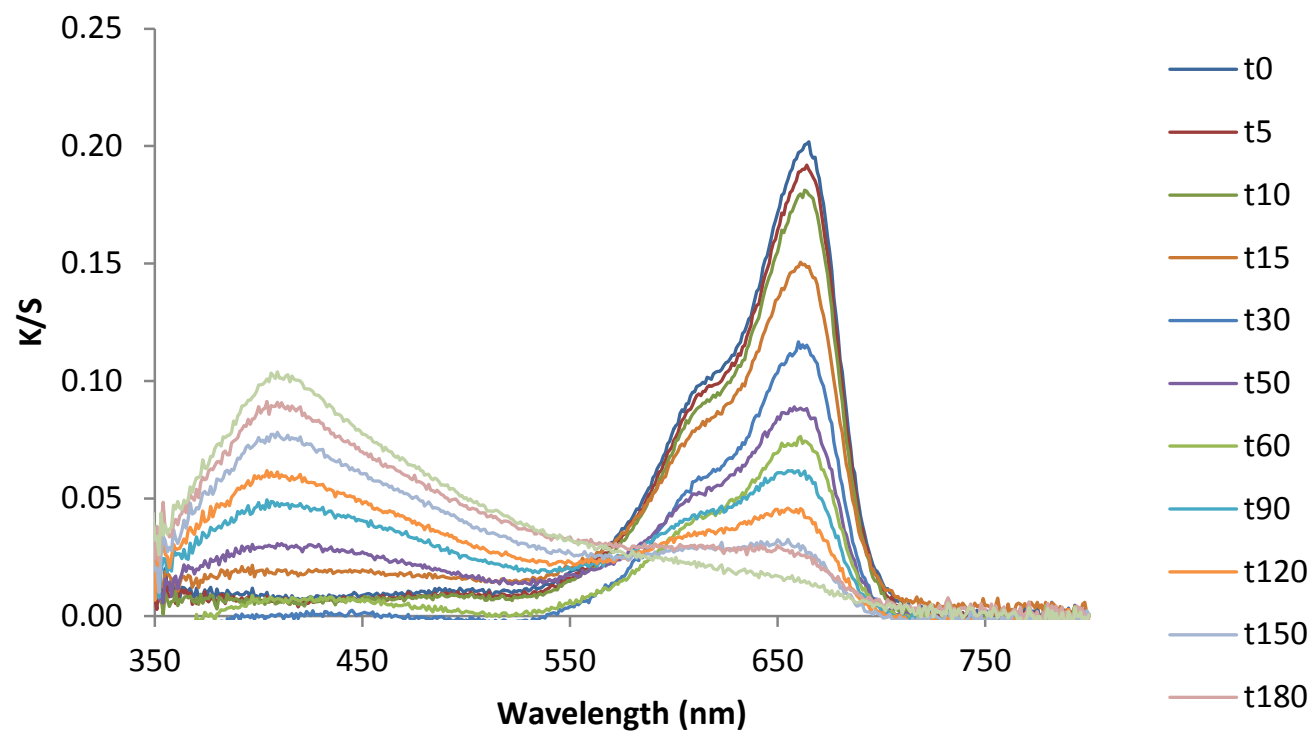

Figure $80 \mathrm{UV} / \mathrm{Vis}$ spectra of $\mathrm{MB}$ with bulk $\mathrm{AgCl}$ in $75 \mathrm{Xe}$ arc lamp overtime (min)

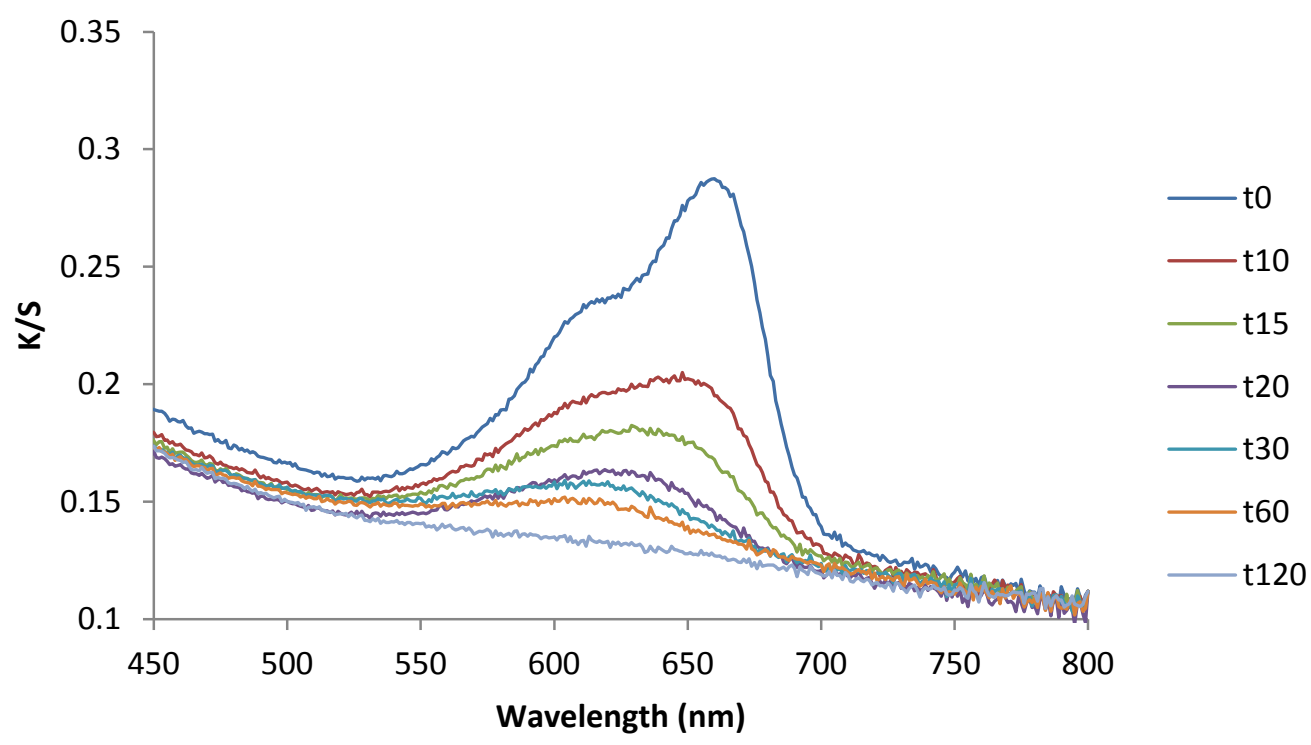

Figure $81 \mathrm{UV} / \mathrm{Vis}$ spectra of $\mathrm{MB}$ with $\mathrm{Ag} / \mathrm{AgCl}$ nanoparticles in $75 \mathrm{~W}$ Xe arc lamp overtime (min) 


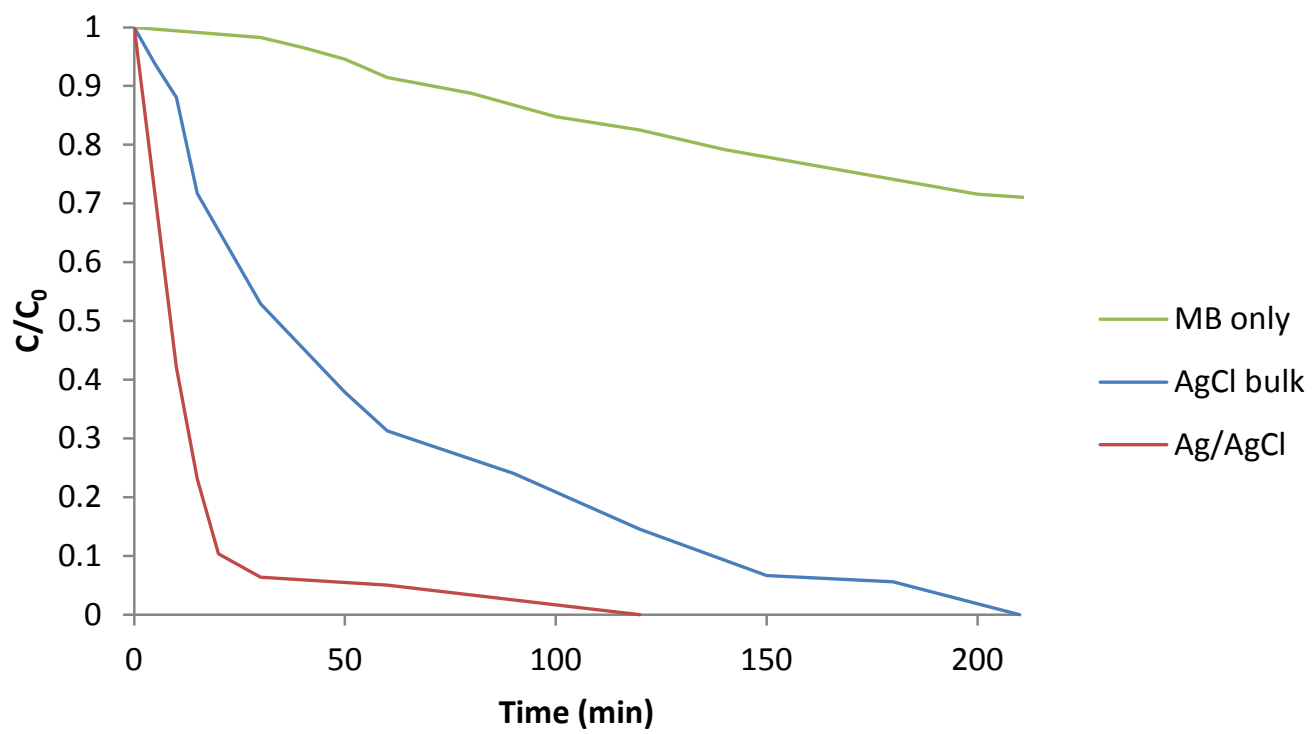

Figure 82 Decrease in MB over time

In the literature it has been previously identified that these silver/silver halide nanoparticles display photocatalytic activity. Generally photocatalytic activity has been established for visible light only (> $400 \mathrm{~nm}$ ) with UV removed using cut off filters. ${ }^{32,42,45,80}$ However, there have been previous examples showing that $\mathrm{Ag} / \mathrm{AgCl}$ nanoparticles have increased photocatalytic activity by comparing $\mathrm{Ag} / \mathrm{AgCl}$ and $\mathrm{AgCl}$. With the $\mathrm{UV}$ cut off filters the $\mathrm{AgCl}$ had negligible effect on the decomposition of dye, as the $\mathrm{AgCl}$ direct and indirect bandgaps lies in the UV $(241 \mathrm{~nm}$ and $382 \mathrm{~nm}) .{ }^{80}$ Even though the photocatalytic experiment used in this research involves $\mathrm{UV}$ and visible light the same trend can be seen, with $\mathrm{Ag} / \mathrm{AgCl}$ nanoparticles exhibiting an increased degradation rate of $\mathrm{MB}$ than $\mathrm{AgCl}$.

After determining that $\mathrm{Ag} / \mathrm{AgCl}$ nanoparticles exhibited increased photocatalytic activity, the nanocomposite samples then needed to be tested for photocatalytic activity. Due to the nature of the porous matrices used for nanocomposite synthesis MB concentration was doubled to allow better monitoring of the MB absorbance by reflectance spectroscopy. Matrix blanks were soaked in $\mathrm{MB}$ overnight under stirring and then irradiated in the $75 \mathrm{~W}$ Xe Arc lamp with the $\mathrm{MB}$ absorbance of the matrix blanks monitored over time by reflectance spectroscopy. The MB dyed wool and kraft fibre matrix blanks showed the same degradation 
rate of $\mathrm{MB}$ due to $\mathrm{UV}$ as seen in the MB solution blank, and so the fibres showed no photocatalytic activity. The photoactivity of the NCS matrix blank will be discussed later. A sample of the $\mathrm{Ag} / \mathrm{AgCl}$ wool nanocomposite was then tested in the same manner. (Figure 83) As seen below in figure 84, the rate of degradation is greatly increased. This shows photocatalytic activity remains in the nanocomposite wool samples. It must be remembered that the dye concentration used in the nanocomposite sample tests is double that previously seen in the $\mathrm{Ag} / \mathrm{AgCl}$ nanoparticle assessment, as such, the time for $\mathrm{MB}$ decomposition is reasonable compared to the $\mathrm{Ag} / \mathrm{AgCl}$ nanoparticles seen earlier.

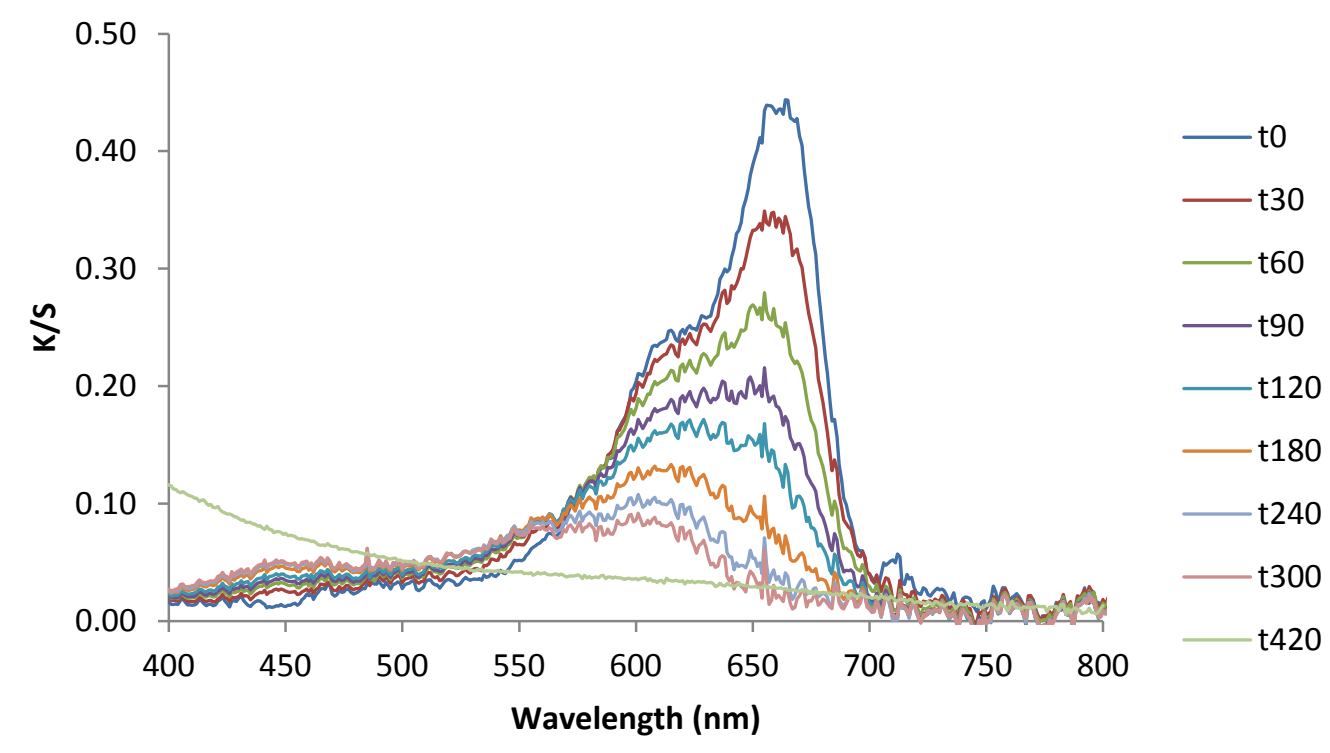

Figure $83 \mathrm{UV} / \mathrm{Vis}$ spectra of $\mathrm{MB}$ with $\mathrm{Ag} / \mathrm{AgCl}$ wool nanocomposite in $75 \mathrm{~W}$ Xe arc lamp overtime (min) 


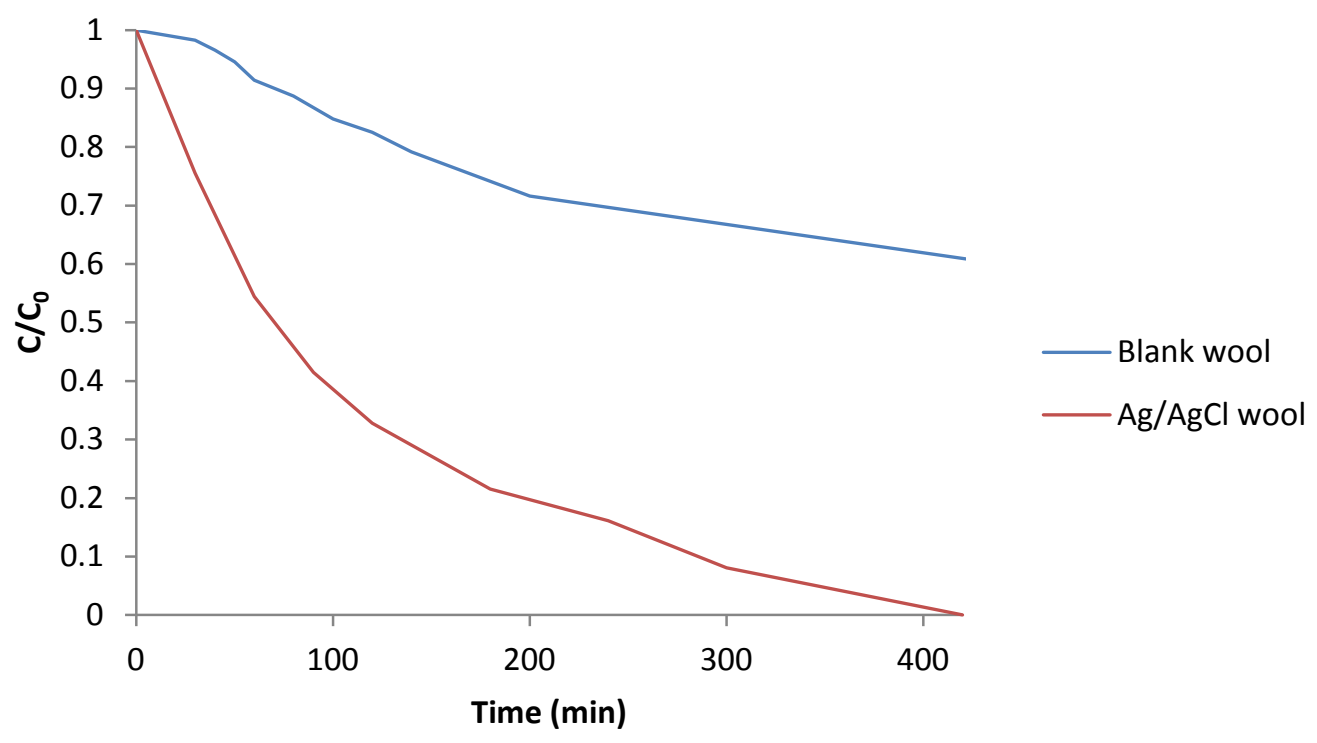

Figure 84 Decrease in MB over time for wool samples

The kraft fibre $\mathrm{Ag} / \mathrm{AgCl}$ nanocomposite photoactivity was also evaluated. Bleached kraft fibres were used for this experiment for as they provided a better signal to noise ratio for the $\mathrm{UV} / \mathrm{Vis}$ spectra giving more resolved peaks with which to monitor the MB. As mentioned blank kraft fibres showed no photocatalytic activity. However, $\mathrm{Ag} / \mathrm{AgCl}$ kraft nanocomposite fibres showed an increased rate of degradation of the $\mathrm{MB}$ dye. The $\mathrm{Ag} / \mathrm{AgCl} \mathrm{kraft}$ nanocompostie degraded the $\mathrm{MB}$ in a similar time frame to that of the $\mathrm{Ag} / \mathrm{AgCl}$ wool nanocomposite. (Figure 85) 


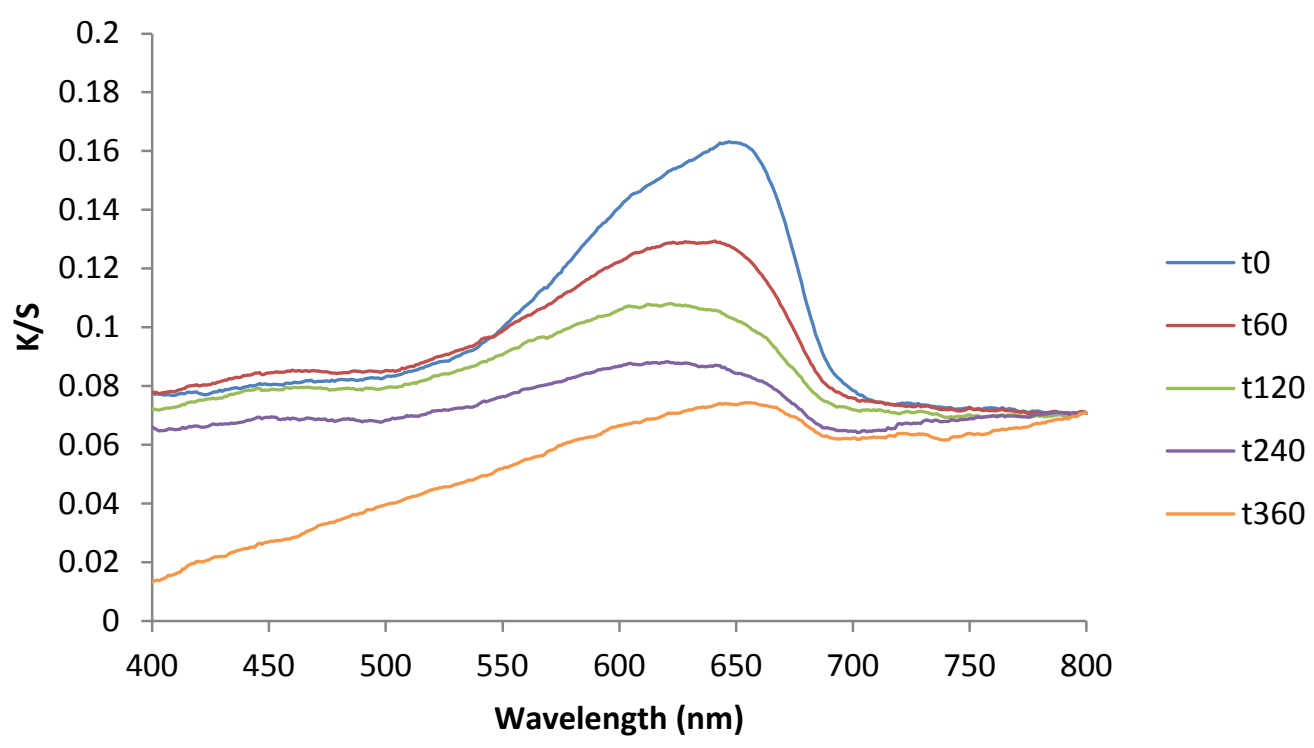

Figure $85 \mathrm{UV} / \mathrm{Vis}$ spectra of $\mathrm{MB}$ with $\mathrm{Ag} / \mathrm{AgCl}$ kraft nanocomposite in $75 \mathrm{~W}$ Xe arc lamp overtime (min)

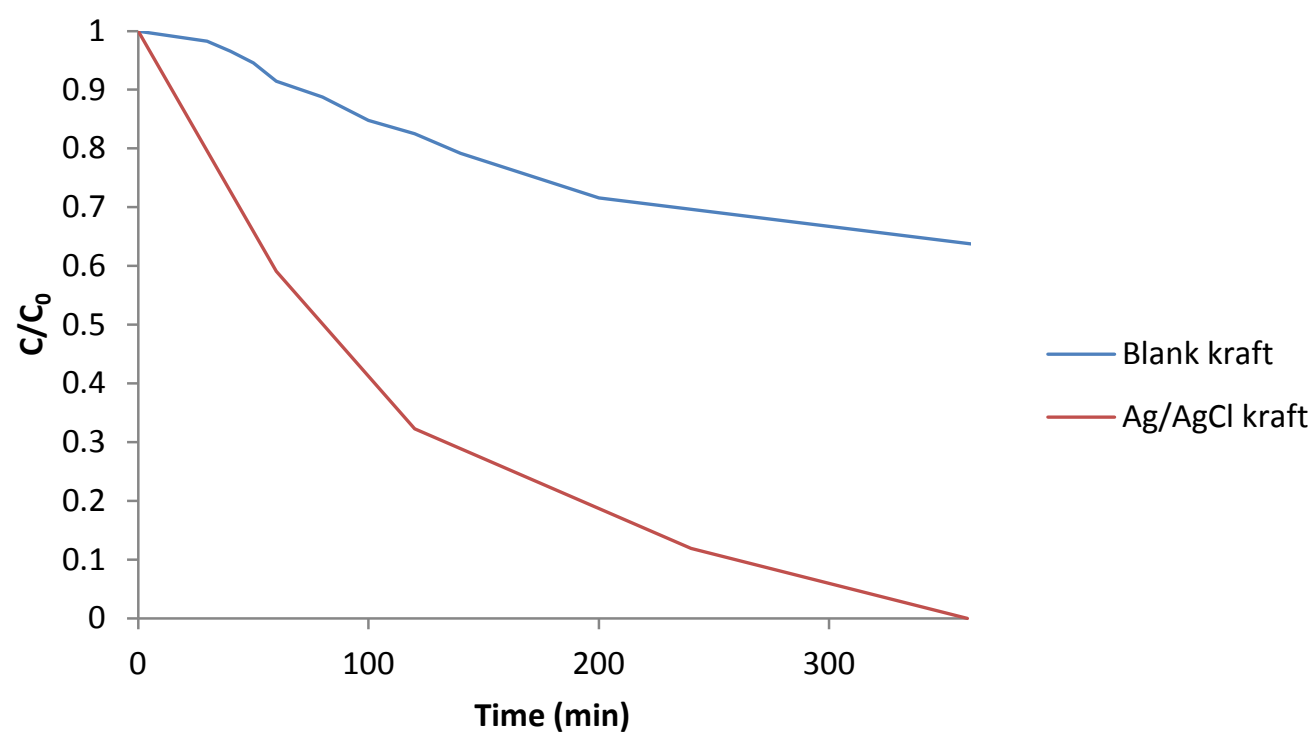

Figure 86 Decrease in MB over time for kraft samples

Finally $\mathrm{Ag} / \mathrm{AgCl}$ NCS nanocomposite was evaluated for photocatalytic activity. Blank NCS dyed with $\mathrm{MB}$ was irraditated and $\mathrm{MB}$ monitored over time to determine if the matrix imparted any photocatalytic activity. In the case of NCS this proved to be true. The NCS 
successfully degrades $\mathrm{MB}$ over a period of 5 hours, this is a greater rate than the $\mathrm{MB}$ degradation in UV/Vis light seen in the other matrix blanks and the MB solution blank, over 24 hours. $\mathrm{Ag} / \mathrm{AgCl} \mathrm{NCS}$ nanocomposites further increased the rate of degradation as seen below. (Figure 87) The MB was degraded over the period of an hour, faster than any of the previous nanocomposites. Remembering that the nanocomposite samples had MB concentrations double the concentration of the $\mathrm{Ag} / \mathrm{AgCl}$ nanoparticles, this shows that the $\mathrm{Ag} / \mathrm{AgCl} \mathrm{NCS}$ nanocomposite has a faster decomposition rate than the nanoparticles alone. Hence,the NCS matrix enhances the photocatalytic activity of the $\mathrm{Ag} / \mathrm{AgCl}$ nanoparticles within the nanocomposite.

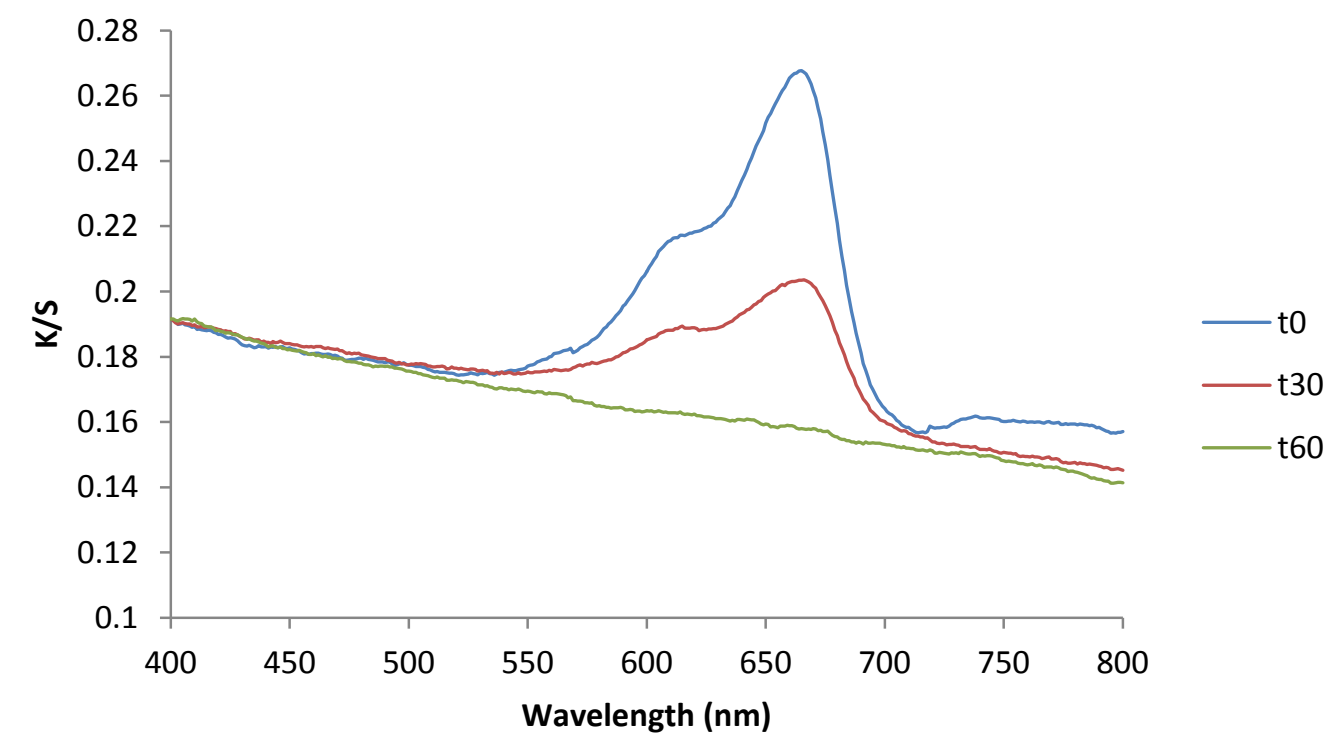

Figure $87 \mathrm{UV} / \mathrm{Vis}$ spectra of $\mathrm{MB}$ with $\mathrm{Ag} / \mathrm{AgCl} \mathrm{NCS}$ nanocomposite in $75 \mathrm{~W}$ Xe arc lamp overtime (min) 


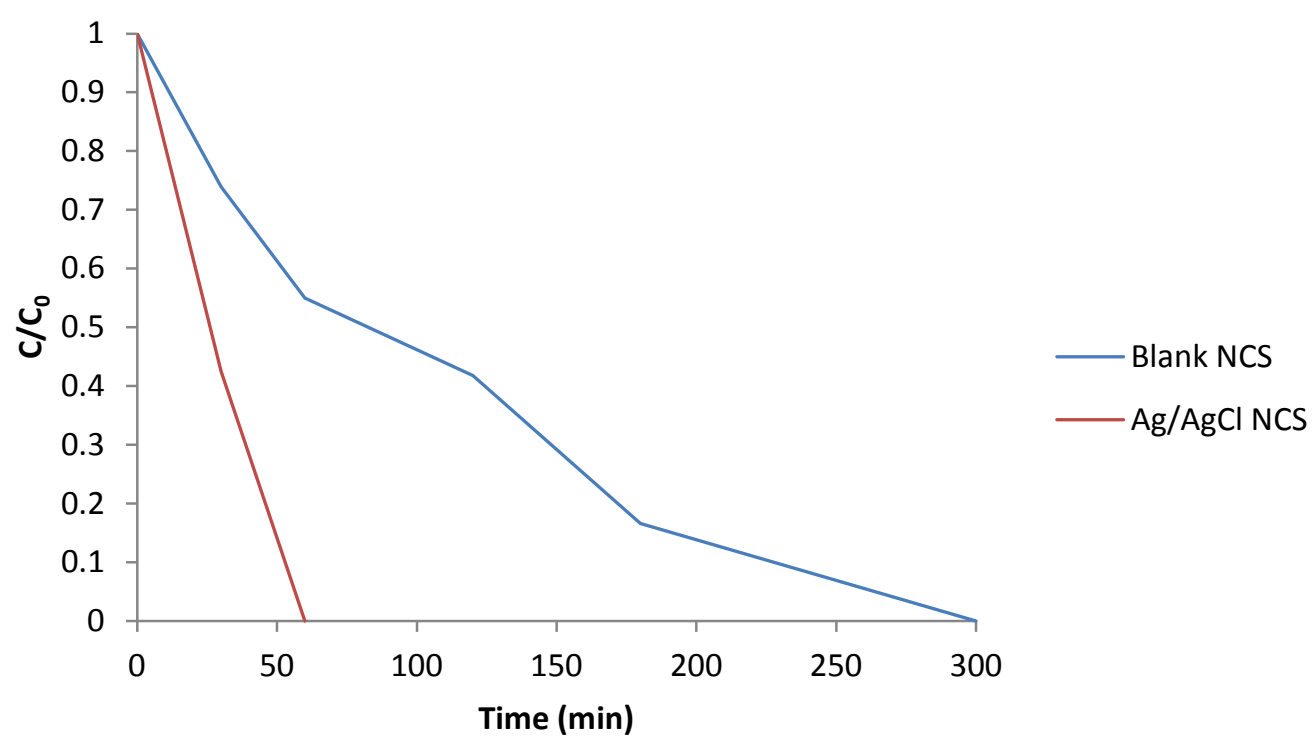

Figure 88 Decrease in MB over time for NCS samples

In summary, although only a preliminary assessment of photocatalytic activity was carried out, it was seen that $\mathrm{Ag} / \mathrm{AgCl}$ nanoparticles display increased photocatalytic properties over bulk $\mathrm{AgCl}$ powder alone. The $\mathrm{Ag} / \mathrm{AgCl}$ nanocomposites all displayed an increased rate of MB degradation with light irradiation. This shows that photocatalytic activity is maintained by the $\mathrm{Ag} / \mathrm{AgCl}$ nanoparticles when incorporated into the nanocomposite matrix. Of note is the $\mathrm{Ag} / \mathrm{AgCl}$ NCS nanocomposite, where the photocatalytic activity of the NCS matrix has enhanced the photocatalytic activity of the $\mathrm{Ag} / \mathrm{AgCl}$ nanoparticles leading to a degradation rate faster than the $\mathrm{Ag} / \mathrm{AgCl}$ nanoparticles alone in solution. 


\section{Conclusions}

This research programme has shown that silver/silver halide nanocomposites have been successfully synthesised using the synthesis procedures outlined in the experimental methods chapter. All three matrices used in this research project; wool, kraft fibres, and nanostructured calcium silicates, were successful in creating silver/silver halide nanocomposites. Silver/silver halide nanoparticles and silver/silver halide nanocomposites were characterised using UV/Vis spectroscopy which showed that with light exposure silver halide nanoparticles underwent partial photocatalysis, forming silver nanodomains. These nanodomains gave rise to a broad visible region absorption in the $\mathrm{AgCl}$ and $\mathrm{AgBr}$ based silver/silver halide nanoparticles. This absorption occurs due to the SPR effects arising from the silver nanodomains. A range of absorption spectra were seen due to the varying size, shape, and agglomeration, of the nanodomains causing a red shift of the SPRB to different degrees. Red shifting of the SPRB is also due to the dielectric constant of the AgX nanoparticles which the silver nanodomains are on. Development of a visible region absorption was not seen in AgI based nanocomposites and nanoparticles. This was due to the low photolysis rate of AgI hindering the self-sensitisation process.

XRD was used to confirm the successful synthesis of silver halide nanoparticles within the nanocomposite matrices. It also showed that crystalline silver was produced after light exposure in $\mathrm{AgCl}$ and $\mathrm{AgBr}$ based nanocomposites and nanoparticles, further confirming silver/silver halide nanoparticle formation. From the XRD and UV/Vis no definitive evidence was seen for the photolysis of $\mathrm{AgI}$, and conversion of $\mathrm{Ag}^{+}$to $\mathrm{Ag}^{0}$. TEM showed two phases present in $\mathrm{Ag} / \mathrm{AgCl}$ nanoparticles, a cubic $\mathrm{AgCl}$ nanoparticle with $\mathrm{Ag}$ nanodomains present on the surface. Electron diffraction, carried out on these nanoparticles, confirmed that both $\mathrm{Ag}$ and $\mathrm{AgCl}$ were present together in the nanoparticle, confirming what was previously seen in the literature. ${ }^{45,77}$

SEM and EDS of the silver/silver halide nanocomposites showed silver halide nanoparticles were distributed throughout the matrices. The nanoparticles were formed within the matrix through a precipitation followed by conversion mechanism. This saw no nanoparticles 
forming on the surface of the wool and kraft fibre matrices, but a ring distribution below the fibre surface due to the migration of halide ions towards the fibre edge to initiate nanoparticle precipitation. The size distribution of the silver/silver halide nanoparticles in NCS was greater as the NCS matrix is very open and porous providing poor size control of the nanoparticles.

The successful synthesis of the silver/silver halide nanocomposites prompted evaluation of their photocatalytic activity with $\mathrm{MB}$ degradation. The $\mathrm{Ag} / \mathrm{AgCl}$ nanoparticles showed increased photocatalytic activity compared to $\mathrm{UV} / \mathrm{V}$ is light degradation and $\mathrm{AgCl}$ powder. The $\mathrm{AgCl}$ wool, kraft and NCS nanocomposites, all showed photocatalytic activity. The nanocomposite materials successfully degraded $\mathrm{MB}$ with light irradiation at higher rates than $\mathrm{UV}$ degradation alone. The $\mathrm{Ag} / \mathrm{AgCl} \mathrm{NCS}$ nanocomposite displayed a synergistic relationship between the $\mathrm{Ag} / \mathrm{AgCl}$ nanoparticles and the NCS matrix, enhancing the photocatalytic activity of the $\mathrm{Ag} / \mathrm{AgCl}$ nanoparticles. Hence, silver/silver halide nanocomposites were successfully synthesised using $\mathrm{AgCl}, \mathrm{AgBr}$ and $\mathrm{AgI}$, on wool, kraft fibre and NCS matrices. These were shown to be photocatalysts. 


\section{Future Perspectives}

It would be useful to continue the evaluation of photocatalytic activity of the nanocomposites and nanoparticles to include $\mathrm{Ag} / \mathrm{AgBr}$ and $\mathrm{Ag} / \mathrm{AgI}$ samples. This would confirm that the photocatalytic behaviour of the silver/silver halide nanoparticles is retained no matter which halide is used in synthesis. It would also allow for comparison of photocatalytic activity of $\mathrm{Ag} / \mathrm{AgCl}, \mathrm{Ag} / \mathrm{AgBr}$ and $\mathrm{Ag} / \mathrm{AgI}$ to determine the most efficient photocatalyst and matrix combination. The literature has also shown that $\mathrm{Ag} / \mathrm{AgCl}, \mathrm{Ag} / \mathrm{AgBr}$ and $\mathrm{Ag} / \mathrm{AgI}$ nanoparticles are also visible light photocatalysts. ${ }^{35,42,44,45}$ This too should be tested, through photocatalytic degradation experiments utilising UV filters to eliminate any contribution of the UV to dye breakdown. Photostability is a property essential for any application of photocatalysts, as repeat usage increases efficiency of the photocatalyst, and often any applications require recyclability. The photostability of the nanocomposites can be explored through repeat photocatalytic activity experiments, taking note of any decreasing rate of decomposition. In order to determine the maximum number of cycles the photocatalyst can be used. Furthermore the mechanism of enhanced photocatalytic activity through the NCS matrix needs to be determined. This could lead to imparting this property onto other matrices, and increasing the photocatalytic activity of the silver/silver halide nanoparticles in this matrix.

The silver halides are known antimicrobial agents, this property was shown to be imparted to the silver/silver halide nanocomposites through the work Kelly. ${ }^{67}$ The kraft paper fibre and NCS nanocomposites may also display antimicrobial activity. This is of value as applications such as waste water treatment air filters and packaging. These applications would find both the antimicrobial nature of the nanocomposites and the photocatalytic activity desirable. 


\section{References}

1. S. Kim, H. Chung, J. H. Kwon, H. G. Yoon and W. Kim, Bulletin of the Korean Chemical Society, 2010, 31, 2918-2922.

2. M. Volkan, D. L. Stokes and T. Vo-Dinh, Sensors and Actuators B-Chemical, 2005, 106, 660-667.

3. T. Tani, Journal of Dispersion Science and Technology, 2004, 25, 375-388.

4. X. Feng, Y. Liu, C. Lu, W. Hou and J.J. Zhu, Nanotechnology, 2006, 17, 3578-3583.

5. M. Ramstedt, B. Ekstrand-Hammarström, A. V. Shchukarev, A. Bucht, L. Österlund, M. Welch and W. T. S. Huck, Biomaterials, 2009, 30, 1524-1531.

6. R. W. Gurney and N. F. Mott, Proceedings of the Royal Society of London Series AMathematical and Physical Sciences, 1938, 164, 151-167.

7. S. Tutihasi, Physical Review, 1957, 105, 882-884.

8. A. I. Gavrilyuk, Technical Physics Letters, 2007, 33, 779-781.

9. G. Burley, Journal of Research of the National Bureau of Standards, 1968, 67A, 301307.

10. P. Monnoyer, A. Fonseca and J. B. Nagy, Colloids and Surfaces A-Physicochemical and Engineering Aspects, 1995, 100, 233-243.

11. M. Husein, E. Rodil and J. Vera, Langmuir, 2003, 19, 8467-8474.

12. M. M. Husein, E. Rodil and J. H. Vera, Journal of Colloid and Interface Science, 2005, 288, 457-467.

13. K. Kimijima and T. Sugimoto, Journal of Colloid and Interface Science, 2005, 286, $520-525$.

14. E. Rodil, L. Aldous, C. Hardacre and M. C. Lagunas, Nanotechnology, 2008, 19, 8.

15. L. Li and Y. J. Zhu, Journal of Colloid and Interface Science, 2006, 303, 415-418.

16. K. Kimijima and T. Sugimoto, Journal of Physical Chemistry B, 2004, 108, 37353738.

17. I. Freestone, N. Meeks, M. Sax and C. Higgitt, Gold Bulletin, 2007, 40, 270-277.

18. A. Muramatsu and Y. Waseda, in Morphology Control of Materials and Nanoparticles, Springer-Verlag Berlin, Berlin, Editon edn., 2004, vol. 64, pp. 129149.

19. G. Mie, Annalen Der Physik, 1908, 25, 377-445.

20. L. M. Liz-Marzan, Materials Today (Oxford, United Kingdom), 2004, 7, 26-31. 
21. P. Prasad, Nanophotonics, Wiley-Interscience, 2004.

22. K. L. Kelly, E. Coronado, L. L. Zhao and G. C. Schatz, Journal of Physical Chemistry $B, 2003$, 107, 668-677.

23. M. R. Hormozi-Nezhad, M. Jalali-Heravi, H. Robatjazi and H. Ebrahimi-Najafabadi, Colloids and Surfaces A: Physicochemical and Engineering Aspects, 2012, 393, 4652.

24. F. Caruso, Colloids and colloid assemblies: synthesis, modification, organization and utilization of colloid particles, Wiley-VCH, 2004.

25. B. P. Rand, P. Peumans and S. R. Forrest, Journal of Applied Physics, 2004, 96, 7519-7526.

26. N. Leopold and B. Lendl, Journal of Physical Chemistry B, 2003, 107, 5723-5727.

27. R. Brause, H. Moltgen and K. Kleinermanns, Applied Physics B-Lasers and Optics, 2002, 75, 711-716.

28. J. J. Mock, M. Barbic, D. R. Smith, D. A. Schultz and S. Schultz, Journal of Chemical Physics, 2002, 116, 6755-6759.

29. I. O. Sosa, C. Noguez and R. G. Barrera, Journal of Physical Chemistry B, 2003, 107, 6269-6275.

30. X. Chen, Chinese Journal of Catalysis, 2009, 30, 839-851.

31. O. Akhavan, Journal of Colloid Interface Science, 2009, 336, 117-124.

32. C. H. An, S. N. Peng and Y. G. Sun, Advanced Materials, 2010, 22, 2570-2574.

33. A. Kafizas, S. Kellici, J. A. Darr and I. P. Parkin, Journal of Photochemistry and Photobiology A: Chemistry, 2009, 204, 183-190.

34. X. B. Chen and C. Burda, Journal of the American Chemical Society, 2008, 130, 5018-5019.

35. P. Wang, B. B. Huang, X. Y. Qin, X. Y. Zhang, Y. Dai, J. Y. Wei and M. H. Whangbo, Angewandte Chemie-International Edition, 2008, 47, 7931-7933.

36. K. Awazu, M. Fujimaki, C. Rockstuhl, J. Tominaga, H. Murakami, Y. Ohki, N. Yoshida and T. Watanabe, Journal of the American Chemical Society, 2008, 130, 1676-1680.

37. Y. P. Bi and J. H. Ye, Chemical Communications, 2009, 6551-6553.

38. J. Cao, B. Luo, H. Lin and S. Chen, Journal of Molecular Catalysis A: Chemical, 2011, 344, 138-144.

39. P. Wang, B. B. Huang, Q. Q. Zhang, X. Y. Zhang, X. Y. Qin, Y. Dai, J. Zhan, J. X. Yu, H. X. Liu and Z. Z. Lou, Chemistry-A European Journal, 2010, 16, 10042-10047. 
40. Z. G. Yi, J. H. Ye, N. Kikugawa, T. Kako, S. X. Ouyang, H. Stuart-Williams, H. Yang, J. Y. Cao, W. J. Luo, Z. S. Li, Y. Liu and R. L. Withers, Nature Materials, 2010, 9, 559-564.

41. H. Zhu, X. Ke, X. Yang, S. Sarina and H. Liu, Angewandte Chemie International Edition, 2010, 49, 9657-9661.

42. J. Jiang and L. Z. Zhang, Chemistry-A European Journal, 2011, 17, 3710-3717.

43. P. Wang, B. B. Huang, X. Y. Zhang, X. Y. Qin, Y. Dai, Z. Y. Wang and Z. Z. Lou, Chemcatchem, 2011, 3, 360-364.

44. P. Wang, B. B. Huang, Z. Z. Lou, X. Y. Zhang, X. Y. Qin, Y. Dai, Z. K. Zheng and X. N. Wang, Chemistry-A European Journal, 2010, 16, 538-544.

45. M. Choi, K. H. Shin and J. Jang, Journal of Colloid and Interface Science, 2009, 341, 83-87.

46. S. Glaus, G. Calzaferri and R. Hoffmann, Chemistry - A European Journal, 2002, 8, 1785-1794.

47. M. Lanz, D. Schürch and G. Calzaferri, Journal of Photochemistry and Photobiology A: Chemistry, 1999, 120, 105-117.

48. L. Kuai, B. Geng, X. Chen, Y. Zhao and Y. Luo, Langmuir, 2010, 26, 18723-18727.

49. C. Hu, T. Peng, X. Hu, Y. Nie, X. Zhou, J. Qu and H. He, Journal of the American Chemical Society, 2009, 132, 857-862.

50. X. Wang, S. Li, H. Yu, J. Yu and S. Liu, Chemistry - A European Journal, 2011, 17, 7777-7780.

51. H. Forward, The Chemical and Physical Structure of Merino Wool, CSIRO.

52. W. Simpson, G. Crawshaw and T. Institute, Wool: science and technology, CRC Press, 2002.

53. M. J. Adams, B. J. Briscoe and T. K. Wee, Journal of Physics D-Applied Physics, 1990, 23, 406-414.

54. P. Greaves and B. Saville, Microscopy of textile fibres, BIOS Scientific in association with the Royal Microscopical Society, 1995.

55. D. Robson, Textile Research Journal, 1997, 67, 747-752.

56. A. P. Negri, H. J. Cornell and D. E. Rivett, Textile Research Journal, 1993, 63, 109115.

57. R. H. Bradley, I. Mathieson and K. M. Byrne, Journal of Materials Chemistry, 1997, 7, 2477-2482. 
58. A. P. Negri, H. J. Cornell and D. E. Rivett, Journal of the Society of Dyers and Colourists, 1993, 109, 296-301.

59. D. J. Evans and M. Lanczki, Textile Research Journal, 1997, 67, 435-444.

60. N. Brack, R. Lamb, D. Pham and P. Turner, Surface and Interface Analysis, 1996, 24, 704-710.

61. R. Kienle, American Association Of Textile Chemists And Colorists, 1945, 42-53.

62. L. Wagner, M. Giesen and H. Zahn, Colloid and Polymer Science, 1983, 261, 365369.

63. J. Leeder, Wool Science Review, 1986, 63, 3-35.

64. M. Feughelman, Mechanical properties and structure of alpha-keratin fibres: wool, human hair and related fibres, UNSW Press, 1997.

65. J. D'Arcy, Sheep Management and Wool Technology, UNSW Press, 1990.

66. L. Hunter, The Angora Goat and Mohair Journal, 1987, 29, 35.

67. F. Kelly, Multifunctional Textiles from New Zealand Wool Coloured with Silver or Silver Halide Nanoparticles, Victoria University of Wellington, 2009.

68. G. A. Smook, Handbook for Pulp and Paper Technologists, 2nd edn., Angus Wilde Publications, Vancouver, 1992.

69. E. Sjöström, Wood Chemistry: Fundamentals and Applications, Academic Press, 1993.

70. J. Johnston and T. Nilsson, Journal of Materials Science, 2012, 47, 1103-1112.

71. J.H. Johnston, T. Borrman, A.J. Mcfarlane, NZ Patent Specification No. 537747 International PCT Application PCT/NZ2006/000003, 2006.

72. M. Cairns, A Study of the Sorption Characteristics of Nanostructured Calcium Silicate, Victoria University of Wellington, 2008.

73. A. McFarlane, The Synthesis and Characterisation of Nano-structured Calcium Silicate, Victoria University of Wellington, 2007.

74. J. H. Johnston, T. Borrmann, D. Rankin, M. Cairns, J. E. Grindrod and A. McFarlane, Current Applied Physics, 2008, 8, 504-507.

75. J. H. Johnston, A. J. McFarlane, T. Borrmann and J. Moraes, Current Applied Physics, 2004, 4, 411-414.

76. A. Zeller, Internship Report, Victoria Universtiy of Wellington, 2007.

77. C. Goessens, D. Schryvers, J. Vanlanduyt and R. Dekeyzer, Ultramicroscopy, 1992, 40, 151-162. 
78. K. Burridge, Gold and Silver Nanoparticles as High Value Colourants and MultiFunctional Entities for Natural Fibres and Minerals, Victoria University of Wellington, 2010.

79. M. S. Zhu, P. L. Chen and M. H. Liu, ACS Nano, 2011, 5, 4529-4536.

80. C. An, R. Wang, S. Wang and X. Zhang, Journal of Materials Chemistry, 2011, 21, 11532-11536. 


\section{Appendix}

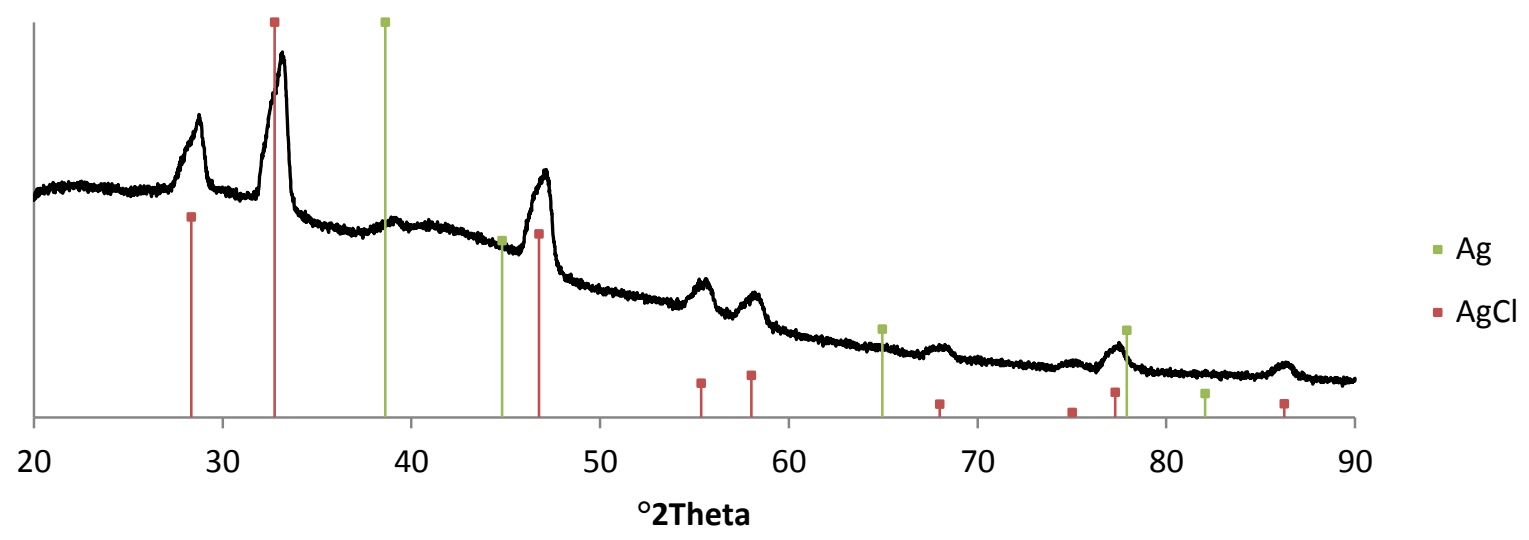

Figure A 1 XRD pattern of AgCl Wool nanocomposite after light exposure (100 ppm)

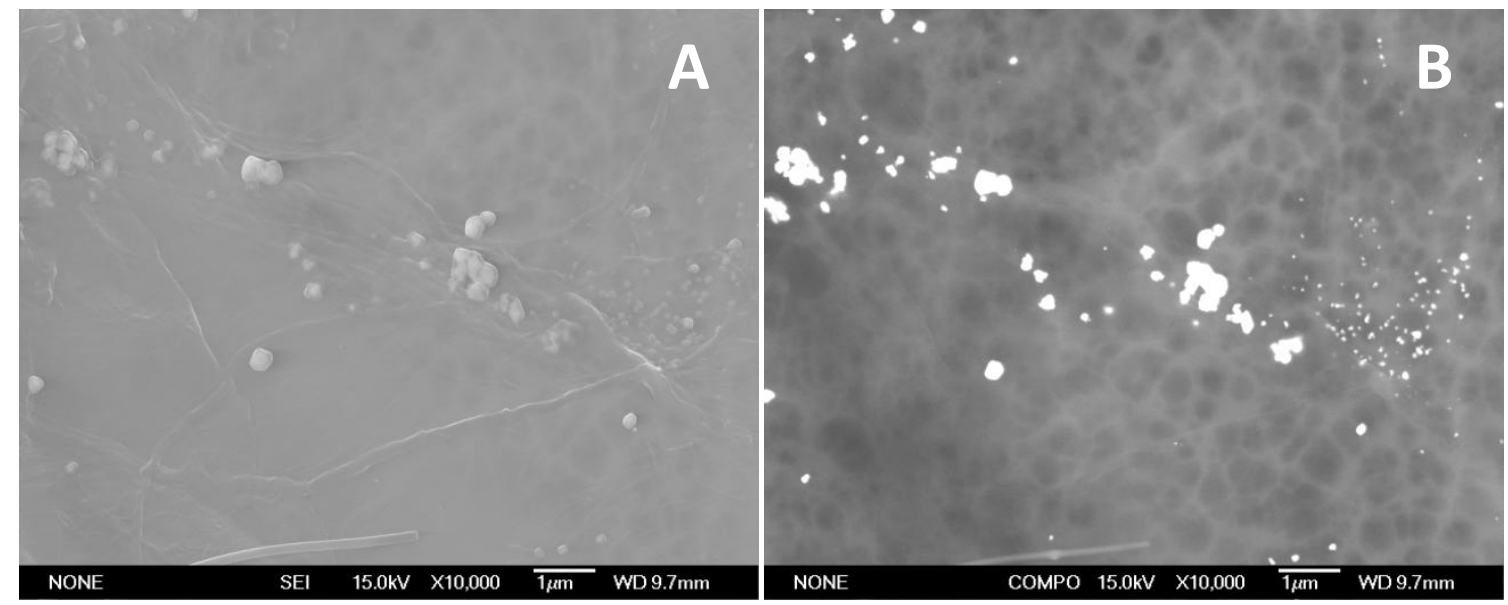

Figure A 2 SEM images of $\mathrm{AgCl}$ bleached kraft fibre nanocomposite A) SEl image B) Backscatter image 

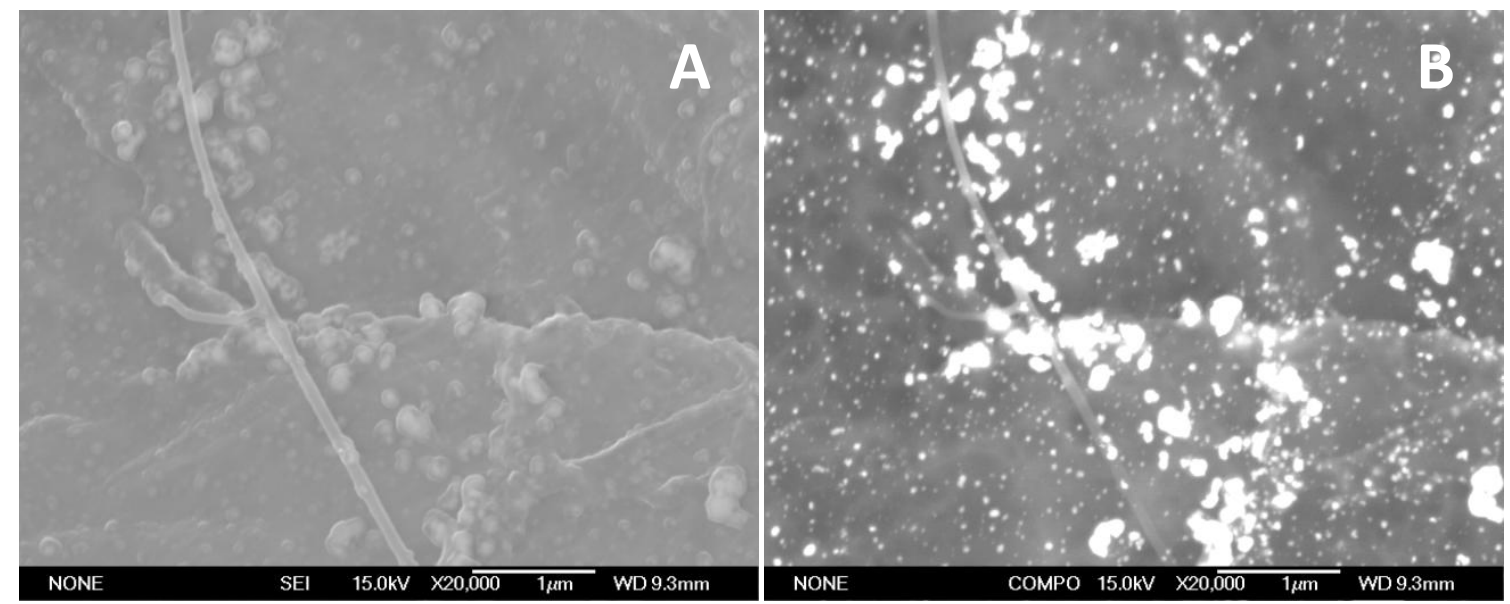

Figure A 3 SEM images of AgBr bleached kraft fibre nanocomposite A) SEl image B) Backscatter image
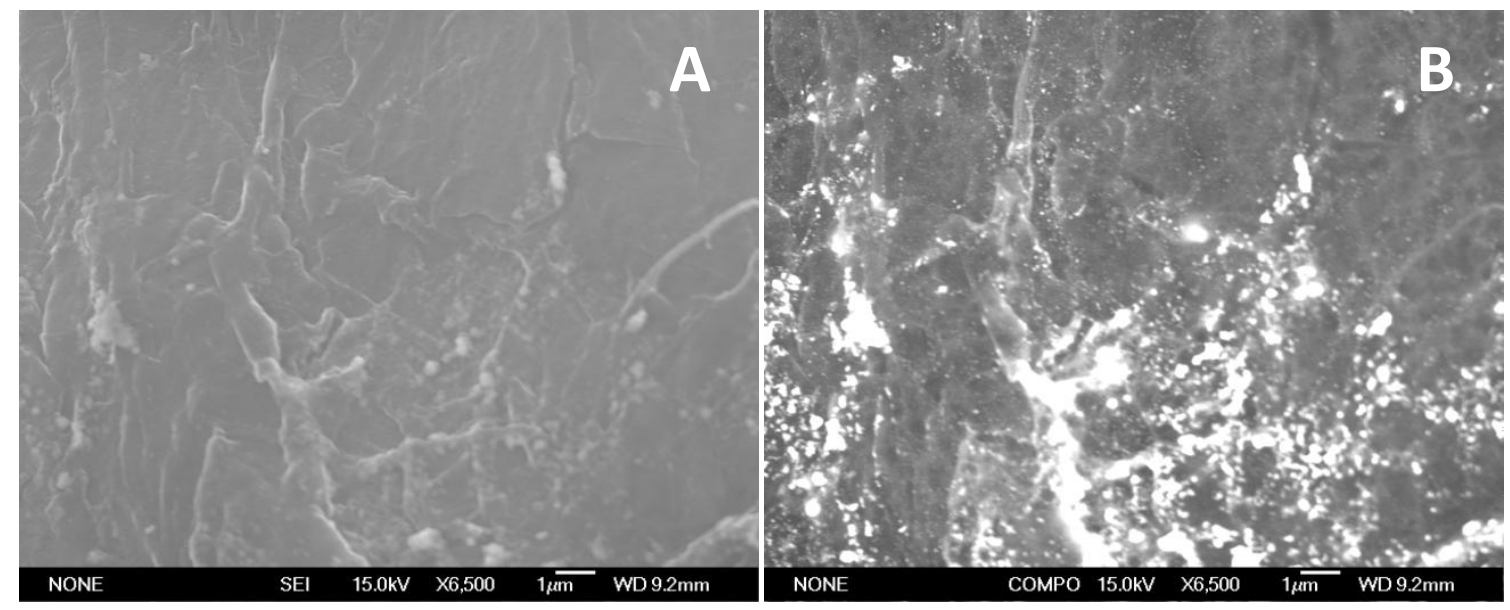

Figure A 4 SEM images of Agl bleached kraft fibre nanocomposite A) SEI image B) Backscatter image 